Acta Math., 224 (2020), 253-388

DOI: 10.4310/ACTA.2020.v224.n2.a2

(C) 2020 by Institut Mittag-Leffler. All rights reserved

\title{
Non-collision singularities in a planar 4-body problem
}

\author{
by \\ JiNXIN XUE \\ Tsinghua University \\ Beijing, China
}

\section{Contents}

1. Introduction . . . . . . . . . . . . . . . . . . . . 254

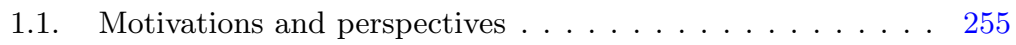

1.2. Sketch of the proof . . . . . . . . . . . . . 257

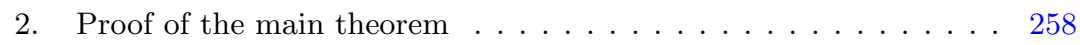

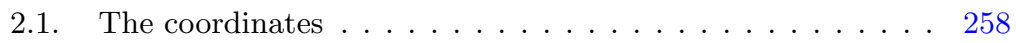

2.2. Gerver's model . . . . . . . . . . . . . . . 260

2.3. The local and global map, the renormalization and the domain . . . . . . . . . . . . . . . . . . . . . 264

2.4. Asymptotics of the local and global map . . . . . . . . . . 266

2.5. The tangent dynamics and the strong expansion . . . . . . 269

2.6. Proof of Theorem 1 . . . . . . . . . . . . . . . . . . 272

3. The hyperbolicity of the Poincaré map . . . . . . . . . . . 276

3.1. The structure of the derivative of the global map and local

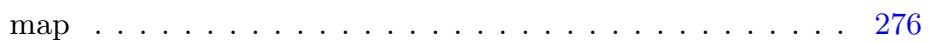

3.2. The non-degeneracy condition . . . . . . . . . . . . 277

3.3. Proof of Lemma 2.17, the expanding cones . . . . . . . . 277

4. Symplectic transformations and Poincaré sections . . . . . . . . . . . . 278

4.1. The Poincaré coordinates . . . . . . . . . . . . . 278

4.2. More Poincaré sections . . . . . . . . . . . . . . . . . . . 279

4.3. Hamiltonian of the right case, when $Q_{4}$ is closer to $Q_{2} \ldots 280$

4.4. Hamiltonian of the left case, when $Q_{4}$ is closer to $Q_{1} \ldots$. . 281

4.5. Hamiltonian of the local map, away from close encounter . 282

4.6. Hamiltonian of the local map, close encounter . . . . . . . 282

5. Statement of the main technical proposition . . . . . . . . . . 283

6. Equations of motion, $\mathscr{C}^{0}$ control of the global map . . . . . . . . 289

6.1. The Hamiltonian equations . . . . . . . . . . . . . . . 289

6.2. Estimates of the Hamiltonian equations . . . . . . . . . . . 290 
6.3. Justification of the assumptions of Lemma $6.5 \ldots \ldots 298$

6.4. Collision exclusion . . . . . . . . . . . . . . 303

6.5. Proofs of Lemmas 2.11 and $2.12 \ldots \ldots$. . . . . . . . 307

6.6. Choosing angular momentum: proof of Lemma 2.21 . . . . 311

7. The variational equation and its solution . . . . . . . . . . . 314

7.1. Derivation of the formula for the boundary contribution . . 315

7.2. Estimates of the variational equation . . . . . . . . . . 316

7.3. Estimates of the solution of the variational equations . . . . 327

8. Estimates of the boundary contribution . . . . . . . . . . . 331

8.1. Boundary contribution for (I) . . . . . . . . . . . . . 331

8.2. Boundary contribution for (III) . . . . . . . . . . . 333

8.3. Boundary contribution for $(\mathrm{V}) \ldots \ldots \ldots 335$

9. Estimates of the matrices (II) and (IV) for switching foci . . . . 335

9.1. A simplifying computation . . . . . . . . . . . . 336

9.2. From Delaunay to Cartesian coordinates . . . . . . . . . . . 336

9.3. From Cartesian to Delaunay coordinates . . . . . . . . . . . . 340

10. The local map . . . . . . . . . . . . . . . . . . . . . . 342

10.1. $\mathscr{C}^{0}$ control of the local map: proof of Lemma $2.10 \ldots 343$

10.2. $\mathscr{C}^{1}$ control of the local map: proof of Lemma $3.1 \ldots \ldots$. . . 347

10.3. Proof of Lemma 3.4 (c) . . . . . . . . . . . . . . . 361

10.4. Proof of Lemma $3.4(\mathrm{a})$ and (b) . . . . . . . . . . 363

Appendix A. Delaunay coordinates . . . . . . . . . . . . . . . . . . 369

A.1. Elliptic motion . . . . . . . . . . . . . . . . . . . . 369

A.2. Hyperbolic motion . . . . . . . . . . . . . . . . . . . 371

A.3. The derivative of Cartesian with respect to Delaunay . . . . 373

A.4. The derivative of Delaunay with respect to Cartesian . . . . 375

A.5. Second-order derivatives . . . . . . . . . . . . . . 376

Appendix B. Gerver's mechanism . . . . . . . . . . . . . . . . . 376

B.1. Gerver's result in $[\mathrm{G} 2] \ldots \ldots \ldots \ldots$. . . . . . . . . 376

Appendix C. $\mathscr{C}^{1}$ control of the global map: proof of Lemma $3.2 \ldots 378$

Acknowledgement . . . . . . . . . . . . . . . . . 387

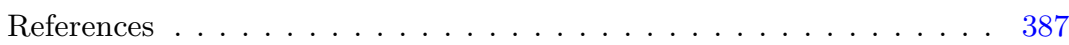

\section{Introduction}

Consider two large bodies $Q_{1}$ and $Q_{2}$ of masses $m_{1}=m_{2}=1$ located at distance $\chi \gg 1$ from each other initially, and two small particles $Q_{3}$ and $Q_{4}$ of masses $m_{3}=m_{4}=\mu \ll 1$. The $Q_{i}$ 's interact with each other via Newtonian potential. We denote the momentum 
of $Q_{i}$ by $P_{i}$. The Hamiltonian of this system can be written as

$$
\begin{aligned}
H\left(Q_{1}, P_{1} ; Q_{2}, P_{2} ; Q_{3}, P_{3} ; Q_{4}, P_{4}\right)=\frac{P_{1}^{2}}{2} & +\frac{P_{2}^{2}}{2}+\frac{P_{3}^{2}}{2 \mu}+\frac{P_{4}^{2}}{2 \mu} \\
& -\frac{1}{\left|Q_{1}-Q_{2}\right|}-\frac{\mu}{\left|Q_{1}-Q_{3}\right|}-\frac{\mu}{\left|Q_{1}-Q_{4}\right|} \\
& -\frac{\mu}{\left|Q_{2}-Q_{3}\right|}-\frac{\mu}{\left|Q_{2}-Q_{4}\right|}-\frac{\mu^{2}}{\left|Q_{3}-Q_{4}\right|} .
\end{aligned}
$$

We choose the mass center as the origin.

We want to study singular solutions of this system, that is solutions which cannot be continued for all positive times. We will exhibit a rich variety of singular solutions. Fix a small $\varepsilon_{0}$. Let $\boldsymbol{\omega}=\left\{\omega_{j}\right\}_{j=1}^{\infty}$ be a sequence of 3's and 4's.

Definition 1.1. We say that $\left(Q_{i}(t), \dot{Q}_{i}(t)\right), i=1,2,3,4$, is a singular solution with symbolic sequence $\boldsymbol{\omega}$ if there exists a positive increasing sequence $\left\{t_{j}\right\}_{j=0}^{\infty}$ such that

- $t^{*}=\lim _{j \rightarrow \infty} t_{j}<\infty$.

- $\left|Q_{3}-Q_{2}\right|\left(t_{j}\right) \leqslant \varepsilon_{0}$ and $\left|Q_{4}-Q_{2}\right|\left(t_{j}\right) \leqslant \varepsilon_{0}$.

- For $t \in\left[t_{j-1}, t_{j}\right]$ we have $\left|Q_{7-\omega_{j}}-Q_{2}\right|(t) \leqslant \varepsilon_{0}$, and $\left\{Q_{\omega_{j}}(t)\right\}_{t \in\left[t_{j-1}, t_{j}\right]}$ leaves the $\varepsilon_{0^{-}}$ neighborhood of $Q_{2}$, winds around $Q_{1}$ exactly once, then reenters the $\varepsilon_{0}$-neighborhood of $Q_{2}$.

- $\lim \sup _{t}\left|\dot{Q}_{i}(t)\right|, \lim \sup _{t}\left|Q_{i}(t)\right| \rightarrow \infty$ as $t \rightarrow t^{*}, i=1,2,3,4$.

During the time interval $\left[t_{j-1}, t_{j}\right]$, we refer to $Q_{\omega_{j}}$ as the traveling particle and to $Q_{7-\omega_{j}}$ as the captured particle. Thus, $\omega_{j}$ prescribes which particle is the traveler during the $j$ th trip.

We denote by $\Sigma_{\boldsymbol{\omega}}$ the set of initial conditions of singular orbits with symbolic sequence $\boldsymbol{\omega}$.

Theorem 1. There exists $\mu_{*} \ll 1$ such that for $\mu<\mu_{*}$ the set $\Sigma_{\boldsymbol{\omega}} \neq \varnothing$. Moreover, there is an open set $U$ on the zero-energy level and zeroth angular momentum level, and a foliation of $U$ by 2-dimensional surfaces such that for any leaf $S$ of our foliation $\Sigma_{\boldsymbol{\omega}} \cap S$ is a Cantor set.

We remark that the choice of the zero-energy level is only for simplicity. Our construction holds for sufficiently small non-zero energy levels.

\subsection{Motivations and perspectives}

Our work is motivated by the following fundamental problem in celestial mechanics. Describe the set of initial conditions of the Newtonian $N$-body problem leading to global solutions. The complement to this set splits into the initial conditions leading to the collision and non-collision singularities. 
It is clear that the set of initial conditions leading to collisions is non-empty for all $N>1$ and it is shown in [Sa1] that it has zero measure. Much less is known about the non-collision singularities. The main motivation for our work is provided by the following basic problems.

Conjecture 1. The set of non-collision singularities has zero measure for all $N>3$.

This conjecture can be found in the problem list [Si] as the first problem. This conjecture remains almost completely open. The only known result, by Saari [Sa2], is that the conjecture is true for $N=4$. To obtain the complete solution of this conjecture one needs to understand better the structure of the non-collision singularities. Our Cantor set in Theorem 1 has zero measure and codimension 2 on the energy level, which is in favor of Conjecture 1. As a first step, it is natural to conjecture the following.

Conjecture 2. (Painlevé Conjecture, 1897) The set of non-collision singularities is non-empty for all $N>3$.

There is a long history studying Conjecture 2. There are some nice surveys, see for instance [G3]. Conjecture 2 was explicitly mentioned in Painlevé's lectures [P], where the author proved that for $N=3$ there are no non-collision singularities, using an argument based on the triangle inequality (see also [G3] for the argument). Soon after Painlevé, von Zeipel showed that if the system of $N$ bodies has a non-collision singularity, then some particle should fly off to infinity in finite time. Thus, non-collision singularities seem quite counterintuitive. The first landmark towards proving the conjecture came in 1975. In [MM] Mather and McGehee constructed a system of four bodies on the line where the particles go to infinity in finite time after an infinite number of binary collisions (it was known since the work of Sundman [Su] that binary collisions can be regularized so that the solutions can be extended beyond the collisions). Since the Mather-McGehee example had collisions, it did not solve Conjecture 2, but made it plausible. Conjecture 2 was proved independently by Xia [Xi] for the spatial 5-body problem and by Gerver [G1] for the planar $3 N$-body problem, where $N$ is sufficiently large. It is a general belief that a non-collision singularity in the $(N+1)$-body problem can be obtained by adding one more remote and light body to the $N$-body problem, to which the existence of non-collision singularities is known. The hardest case of the problem, $N=4$, still remained open. Our result proves the conjecture in the $N=4$ case.

We believe the method used in this paper could also be used to construct noncollision singularities for the general $N$-body problem, for any $N>3$. We can put any number of bodies into our system sufficiently far from the mass center of our four bodies, orthogonal to the line passing through $Q_{1}$ and $Q_{2}$. This produces non-collision singularities in the $N$-body problem. We have not checked all the details in that case, but we do 
not expect any significant difficulties. Treating the general $N$ however would significantly increase the length of the paper, so to simplify the exposition we concentrate here on the 4-body case.

Since our technique is perturbative and it is necessary that $\mu \ll 1$, we ask the following questions.

Question 1. Are there non-collision singularities for the 4-body problem in which all the four bodies have comparable masses?

In fact it is possible that the following stronger result holds.

Question 2. Is it true that for any choice of positive masses $\left(m_{1}, m_{2}, m_{3}, m_{4}\right) \in \mathbb{R} \mathbb{P}^{3}$ the corresponding 4-body problem has non-collision singularities?

We need to develop some non-perturbative techniques for the first question and we need to explore the obstructions for the existence of non-collision singularities for the second.

\subsection{Sketch of the proof}

The proof consists of the following three aspects: physical, mathematical and algorithmic aspects. The physical aspect is an idealistic model constructed by Gerver [G2] (see §2.2), in which the hyperbolic Kepler motion of one light body can extract energy from the elliptic Kepler motion of the other light body. Moreover, after each cycle of energy extraction, the configuration is made self-similar to the beginning, so that the procedure of energy extraction can be iterated infinitely.

The mathematical aspect is a partially hyperbolic dynamics framework. We find that there are two strongly expanding directions that are invariant under iterates along our singular orbits. The strong expansions allow us to push the iteration to the future and synchronize the two light bodies. Namely, the two light bodies can be chosen to come to the correct place simultaneously in order to have a close encounter. One strong expansion is given by a close encounter between $Q_{1}$ and $Q_{4}$. This is the hyperbolicity created by scattering (hyperbolic Kepler motion). The other one is induced by shear coming from the elliptic Kepler motion, which seems quite new in celestial mechanics.

The algorithmic aspect is a systematic toolbox that we develop to compute the derivative of the Poincaré map in detail. This toolbox includes symplectic coordinate systems and partition of the phase space ( $\S 4$ and Appendix A), integration of the variational equations $(\S 7)$ and boundary contributions $(\S 8)$, coordinate change between different pieces of the phase space $(\S 9)$, collision exclusion ( $(6.4)$, etc. Moreover, we develop new methods to regularize the double collision using hyperbolic Delaunay coordinates and extract $\mathscr{C}^{1}$ 
information of the near double collision from its singular limit, the elastic collision, using polar coordinates $(\S 10)$. These new methods are more suitable to our framework than previously known methods such as Levi-Civita regularization, and hopefully have wider applications.

The paper is organized as follows. In $\S 2$, we give the proof of the main Theorem 1. In $\S 3$ we study the structure of the derivative of the local map and the global map. In $\S 4$, we perform several symplectic transformations to reduce the Hamiltonian system to a form suitable for doing calculations and estimates. This section is purely algebraic without dynamics. Next, we state our estimates for the derivatives of the factor maps of the global map as Proposition 5.2 in $\S 5$. The following $\S \S 6-10$ are devoted to the proof of the proposition. In Appendix C, we give the proof of our main estimate for the derivative of the global map, Lemma 3.2, based on Proposition 5.2. Finally, in Appendix A, we give an introduction to Delaunay variables including estimates of the various partial derivatives which are used in our calculations, and in Appendix B, we summarize the result of Gerver in [G2].

We use the following conventions for constants:

- We use $C, c, \widehat{C}, \widetilde{C}$ (without subscript) to denote a constant whose value may be different in different contexts.

- When we use subscript $1,3,4$, for instance $C_{1}, C_{3}, C_{4}$, etc., we mean the constant has fixed value throughout the paper specifically chosen for the first, third or fourth body.

\section{Proof of the main theorem}

\subsection{The coordinates}

We first introduce the set of coordinates needed to state our lemmas and prove our theorems. This set of coordinates is known as the Jacobi coordinates.

Definition 2.1. (The coordinates) - We define the relative position of $Q_{1}, Q_{3}$ and $Q_{4}$ to $Q_{2}$ as the new variables $q_{1}, q_{3}, q_{4}$ :

$$
q_{1}=Q_{1}-Q_{2}, \quad q_{3}=Q_{3}-Q_{2}, \quad q_{4}=Q_{4}-Q_{2},
$$

and the new momentum $p_{1}, p_{3}, p_{4}$, which are related to the old momentum $P_{1}, P_{3}, P_{4}$ by

$$
P_{1}=\mu p_{1}, \quad P_{3}=\mu p_{3}, \quad P_{4}=\mu p_{4} .
$$


- Next, we define the new set of variables $\left(x_{3}, v_{3} ; x_{1}, v_{1} ; x_{4}, v_{4}\right)$ called Jacobi coordinates through

$$
\left\{\begin{array} { l } 
{ v _ { 3 } = p _ { 3 } + \frac { \mu } { 1 + \mu } ( p _ { 4 } + p _ { 1 } ) , } \\
{ v _ { 1 } = p _ { 1 } , } \\
{ v _ { 4 } = p _ { 4 } + \frac { \mu p _ { 1 } } { 1 + 2 \mu } , }
\end{array} \quad \left\{\begin{array}{l}
x_{3}=q_{3}, \\
x_{1}=q_{1}-\frac{\mu\left(q_{3}+q_{4}\right)}{2 \mu+1}, \\
x_{4}=q_{4}-\frac{\mu q_{3}}{1+\mu}
\end{array}\right.\right.
$$

One can easily check that this transformation is symplectic, i.e. the following symplectic form $\bar{\omega}$ is preserved:

$$
\bar{\omega}=\sum_{i=3,1,4} d p_{i} \wedge d q_{i}=\sum_{i=3,1,4} d v_{i} \wedge d x_{i} .
$$

- The total angular momentum is

$$
G_{0}:=\sum_{i=3,1,4} p_{i} \times q_{i}=\sum_{i=3,1,4} v_{i} \times x_{i} .
$$

In this paper we assume the total angular momentum $G_{0}=0$.

Remark 2.2. - This set of new coordinates $\left(x_{3}, v_{3} ; x_{1}, v_{1} ; x_{4}, v_{4}\right)$ looks complicated. Heuristically, the new coordinates have the same physical meanings as the old coordinates $\left(q_{3}, p_{3} ; q_{1}, p_{1} ; q_{4}, p_{4}\right)$, since the transformation between them is a $O(\mu)$ perturbation of Id. We will study coordinate changes systematically in $\S 4$.

- The rescaling (2.2) changes the meanings of some physical quantities. First, $v_{3}$ and $v_{4}$ are close to the velocities of $Q_{3}$ and $Q_{4}$, respectively; however, $v_{1}$ is not close to the velocity of $Q_{1}$ but is close to $\mu^{-1}$ times the velocity of $Q_{1}$. Next, the angular momentum $G_{0}$ that we use here is actually $\mu^{-1}$ times the angular momentum defined using the original coordinates $P_{i}$ and $Q_{i}, i=1,2,3,4$. Similarly, the energy is also $\mu^{-1}$ times the original energy.

We then use Appendix A to pass to Delaunay variables $\left(x_{3}, v_{3}\right) \mapsto\left(L_{3}, \ell_{3}, G_{3}, g_{3}\right)$ and $\left(x_{4}, v_{4}\right) \mapsto\left(L_{4}, \ell_{4}, G_{4}, g_{4}\right)$. For Kepler motion with Hamiltonian

$$
H_{2}=\frac{|v|^{2}}{2}-\frac{1}{|x|}, \quad(x, v) \in \mathbb{R}^{2} \times \mathbb{R}^{2},
$$

the Delaunay variables have explicit geometric meanings. When $H_{2}<0$, the Kepler motion is elliptic. The quantity $L^{2}$ is the semimajor axis, $|L G|$ is the semi-minor axis, $g$ is the argument of apapsis, and $\ell$ is the mean anomaly indicating the position of the moving particle on the ellipse. When $H_{2}>0$, the Kepler motion is hyperbolic, in which 
case the Delaunay variables have similar geometric meanings. Details are provided in Appendix A.

To start, we assume the energy $E_{3}$ of the subsystem $\left(x_{3}, v_{3}\right)$ is negative, while the energy $E_{4}$ of the subsystem $\left(x_{4}, v_{4}\right)$ is positive. The energies and their relations to the Delaunay variables are given as follows:

$$
E_{3}:=\frac{\left|v_{3}\right|^{2}}{2 m_{3}}-\frac{k_{3}}{\left|x_{3}\right|}=-\frac{m_{3} k_{3}^{2}}{2 L_{3}^{2}} \quad \text { and } \quad E_{4}:=\frac{\left|v_{4}\right|^{2}}{2 m_{4}}-\frac{k_{4}}{\left|x_{4}\right|}=\frac{m_{4} k_{4}^{2}}{2 L_{4}^{2}},
$$

where the values of $m_{i}$ and $k_{i}$ are given explicitly in (4.5) below, and it is enough to know that $m_{i}, k_{i}=1+O(\mu), i=3,4$. The variable $G_{i}=v_{i} \times x_{i}$ means minus the angular momentum of the subsystem $\left(x_{i}, v_{i}\right), i=3,4$.

We fix the zero-energy level so that we can eliminate $L_{4}$ from our list of variables, applying the implicit function theorem (§6.1). Next we pick a Poincaré section and treat $\ell_{4}$ as the new time (see Definition 2.6 below), so that we eliminate $\ell_{4}$ from our set of coordinates. So we get $\left(L_{3}, \ell_{3}, G_{3}, g_{3} ; x_{1}, v_{1} ; G_{4}, g_{4}\right) \in \mathbb{R}^{7} \times \mathbb{T}^{3}$ as the set of coordinates that we use to do calculations. In this section, we use the energy $E_{3}$ instead of $L_{3}$, eccentricities $e_{3}$ and $e_{4}$ instead of the negative angular momentum $G_{3}$ and $G_{4}$. The new choice of coordinates are related to the old ones through $e_{i}=\sqrt{1+2 G_{i}^{2} E_{i}}, i=3,4$. We use the set of coordinates $\left(E_{3}, \ell_{3}, e_{3}, g_{3} ; x_{1}, v_{1} ; e_{4}, g_{4}\right)$ to give the proof of the main theorem, since it is easier to study their behavior under the rescaling. Actually, our system still has total angular momentum conservation. We could have fixed an angular momentum and eliminated two more variables. However, this would lead to more complicated calculations.

Notation 2.3. • We refer to our set of variables as

$$
\mathcal{V}=\left(\mathcal{V}_{3} ; \mathcal{V}_{1} ; \mathcal{V}_{4}\right)=\left(L_{3}, \ell_{3}, G_{3}, g_{3} ; x_{1}, v_{1} ; G_{4}, g_{4}\right) \text {. }
$$

- We denote the Cartesian variables as

$$
\mathcal{X}:=\left(\mathcal{X}_{3} ; \mathcal{X}_{1} ; \mathcal{X}_{4}\right)=\left(x_{3}, v_{3} ; x_{1}, v_{1} ; x_{4}, v_{4}\right) .
$$

- In the following, when we use Cartesian coordinates such as $x$ and $v$, each letter has two components. We will use the subscript $\|$ to denote the horizontal coordinate and subscript $\perp$ to denote the vertical coordinate. So we write $x=\left(x_{\|}, x_{\perp}\right), v=\left(v_{\|}, v_{\perp}\right)$, etc.

\subsection{Gerver's model}

Following [G2], we discuss in this section the dynamics of the subsystem $Q_{2}, Q_{3}, Q_{4}$ in the limit case $\mu=0$ with $Q_{1}$ ignored. We assume that

- $Q_{3}$ has elliptic motion and $Q_{4}$ has hyperbolic motion with focus $Q_{2}$; 


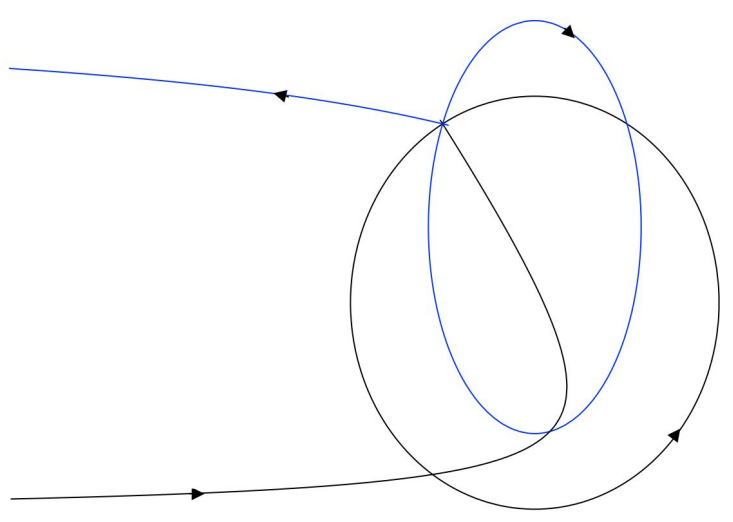

Figure 1. Angular momentum transfer.

- $Q_{3}$ and $Q_{4}$ arrive at the correct intersection point of their orbits simultaneously (see Figures 1 and 2);

- $Q_{3}$ and $Q_{4}$ do not interact unless they have an exact collision, and the collision is treated as elastic collision (energy and momentum are preserved).

The main conclusion is that

- the major axis of the elliptic motion is always kept vertical;

- the incoming and outgoing asymptotes of the hyperbolic motion are always horizontal;

- after two steps of the collision procedure, the ellipse has the same eccentricity as the ellipse before the first collision, but has a smaller semi-major axis (see Figures 1 and 2).

The interaction of $Q_{3}$ and $Q_{4}$ is desribed by the elastic collision. That is, velocities before $(-)$ and after $(+)$ the collision are related by

$$
v_{3}^{+}=\frac{v_{3}^{-}+v_{4}^{-}}{2}+\left|\frac{v_{3}^{-}-v_{4}^{-}}{2}\right| n(\alpha) \quad \text { and } \quad v_{4}^{+}=\frac{v_{3}^{-}+v_{4}^{-}}{2}-\left|\frac{v_{3}^{-}-v_{4}^{-}}{2}\right| n(\alpha),
$$

where $n(\alpha)$ is a unit vector making angle $\alpha$ with $v_{3}^{-}-v_{4}^{-}$. The only free parameter $\alpha$ here is fixed by the condition that the outgoing asymptote of the traveling particle is horizontal.

We next introduce the Gerver map to formalize the above description. The Gerver map describes the parameters of the elliptic orbit change during the interaction of $Q_{3}$ and $Q_{4}$. The orbits of $Q_{3}$ and $Q_{4}$ intersect in two points, of which we pick one (see 


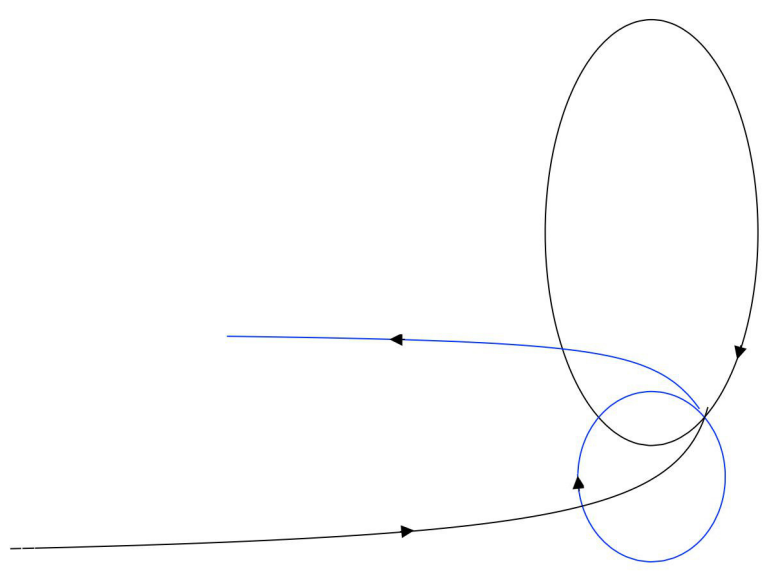

Figure 2. Energy transfer.

Figures 1 and 2). We use the subscript $j \in\{1,2\}$ to describe the first or the second collision in Gerver's construction. Since $Q_{1}$ is ignored, we use only the orbit parameters $\left(E_{3}, \ell_{3}, e_{3}, g_{3} ; e_{4}, g_{4}\right)$. The assumptions on the horizontal asymptotes of the traveler further remove $g_{4}$. Finally, at the intersection point of the elliptic and hyperbolic orbit, we get rid of one last variable $\ell_{3}$, so we only need to work with the variables $\left(E_{3}, e_{3}, g_{3}, e_{4}\right)$.

With this in mind, we proceed to define the Gerver map $\mathbf{G}_{e_{4}, j, \omega}\left(E_{3}, e_{3}, g_{3}\right)$. This map depends on two discrete parameters $j \in\{1,2\}$ and $\omega \in\{3,4\}$. The role of $j$ has been explained above, and $\omega$ will tell us which particle will be the traveler after the collision. $Q_{4}$ moves on an orbit with parameters $\left(\bar{E}_{4}, \bar{e}_{4}, \bar{g}_{4}\right)$.

If $\omega=4$, we choose $\alpha$ so that after the exchange $Q_{4}$ moves on a hyperbolic orbit with horizontal asymptote and let

$$
\mathbf{G}_{e_{4}, j, 4}\left(E_{3}, e_{3}, g_{3}\right)=\left(\bar{E}_{3}, \bar{e}_{3}, \bar{g}_{3}\right) .
$$

If $\omega=3$, we choose $\alpha$ so that after the exchange $Q_{3}$ moves on a hyperbolic orbit with horizontal asymptote and let

$$
\mathbf{G}_{e_{4}, j, 3}\left(E_{3}, e_{3}, g_{3}\right)=\left(\bar{E}_{4}, \bar{e}_{4}, \bar{g}_{4}\right) .
$$

In the following, to fix our notation, we always call the captured particle $Q_{3}$ and the traveler $Q_{4}$, i.e. we fix $\omega=4$.

We will denote the ideal orbit parameters in Gerver's paper [G2] of $Q_{3}$ and $Q_{4}$ before the first (resp. second) collision with * (resp. $\left.{ }^{* *}\right)$. Thus, for example, $G_{4}^{* *}$ will denote 
the negative angular momentum of $Q_{4}$ before the second collision. The real values after the first (resp. after the second) collisions are denoted with a bar or double bar.

The following is the main result of [G2] and plays a key role in constructing singular solutions.

Lemma 2.4. ([G2], [DX, Lemma 2.2]) Assume that the total energy of the $Q_{2}, Q_{3}, Q_{4}$ system is zero, i.e. $E_{3}+E_{4}=0$, and fix the incoming and outgoing asymptotes of the hyperbola to be horizontal.

(a) For $E_{3}^{*}=-\frac{1}{2}, g_{3}^{*}=\frac{1}{2} \pi$ and for any $e_{3}^{*} \in\left(0, \frac{1}{2} \sqrt{2}\right)$, there exist $e_{4}^{*}, e_{4}^{* *}, \lambda_{0}>1$ such that

$$
\left(e_{3}, g_{3}, E_{3}\right)^{* *}=\mathbf{G}_{e_{4}^{*}, 1,4}\left(e_{3}, g_{3}, E_{3}\right)^{*} \quad \text { and } \quad\left(e_{3},-g_{3}, \lambda_{0} E_{3}\right)^{*}=\mathbf{G}_{e_{4}^{* *}, 2,4}\left(e_{3}, g_{3}, E_{3}\right)^{* *},
$$

where $E_{3}^{* *}=E_{3}^{*}=-\frac{1}{2}, g_{3}^{* *}=g_{3}^{*}=\frac{1}{2} \pi$ and $e_{3}^{* *}=\sqrt{1-e_{3}^{* 2}}$.

(b) There exists a constant $\bar{\delta}$ such that, if $\left|\left(e_{3}, g_{3}, E_{3}\right)-\left(e_{3}^{*}, g_{3}^{*}, E_{3}^{*}\right)\right|<\bar{\delta}$, then there exist smooth functions $e_{4}^{\prime}\left(e_{3}, g_{3}\right), e_{4}^{\prime \prime}\left(e_{3}, g_{3}\right)$ and $\lambda\left(e_{3}, g_{3}, E_{3}\right)$ such that

$$
e_{4}^{\prime}\left(e_{3}^{*}, g_{3}^{*}\right)=e_{4}^{*}, \quad e_{4}^{\prime \prime}\left(e_{3}^{*}, g_{3}^{*}\right)=e_{4}^{* *}, \quad \lambda\left(e_{3}^{*}, g_{3}^{*}, E_{3}^{*}\right)=\lambda_{0},
$$

and

$$
\begin{aligned}
\left(\bar{e}_{3}, \bar{g}_{3}, \bar{E}_{3}\right) & =\mathbf{G}_{e_{4}^{\prime}\left(e_{3}, g_{3}\right), 1,4}\left(e_{3}, g_{3}, E_{3}\right), \\
\left(e_{3}^{*},-g_{3}^{*}, \lambda\left(e_{3}, g_{3}, E_{3}\right) E_{3}^{*}\right) & =\mathbf{G}_{e_{4}^{\prime \prime}\left(e_{3}, g_{3}\right), 2,4}\left(\bar{e}_{3}, \bar{g}_{3}, \bar{E}_{3}\right) .
\end{aligned}
$$

(c) (1-homogeneity in $\left.E_{3}\right)$ for any $\lambda>0$ and $\left(e_{3}, g_{3}, E_{3}\right)$ such that

$$
\left\|\left(e_{3}, g_{3}, \frac{E_{3}}{\lambda}, e_{4}\right)-\left(e_{3}, g_{3}, E_{3}, e_{4}\right)^{\dagger}\right\|_{\infty}<\bar{\delta},
$$

with $\dagger=*, * *$, we have

$$
\pi_{E_{3}} \mathbf{G}_{e_{4}, j, 4}\left(e_{3}, g_{3}, E_{3}\right)=\lambda \cdot \pi_{E_{3}} \mathbf{G}_{e_{4}, j, 4}\left(e_{3}, g_{3}, \frac{E_{3}}{\lambda}\right),
$$

where $\pi_{E_{3}}$ means the projection to the $E_{3}$ component, and $j=1,2$ corresponds to $*, * *$.

Part (a) is the main content of [G2], which gives a 2-step procedure to decrease the energy of the elliptic Kepler motion and maintain the self-similar structure (see Figures 1 and 2). We call the collision points in part (a) the Gerver's collision points, whose exact coordinates can be found in Appendix B. The results are summarized in Appendix B with orbit parameters given explicitly. Part (b) says that once the ellipse gets deformed slightly away from the standard case in Figure 1 after the first collision, we can correct 
it by changing the phase of $Q_{3}$ slightly at the next collision to guarantee that the ellipse that we get after the second collision is standard.

The notion of angle of asymptote above is clear since we only deal with the Kepler motion. We next introduce the explicit definition of angles of asymptotes, which are used in place of $g_{4}$ sometimes even when we deal with perturbed Kepler motion.

Notation 2.5. (Angles of asymptotes) In the following, we use

$$
\theta_{4}^{-}:=g_{4}-\arctan \left(\frac{G_{4}}{L_{4}}\right)
$$

for the incoming (superscript -) asymptote of the $\left(x_{4}, v_{4}\right)$ motion and

$$
\theta_{4}^{+}:=\pi+g_{4}+\arctan \left(\frac{G_{4}}{L_{4}}\right)
$$

for the outgoing (superscript + ) asymptote. In Lemma 2.4, we always have $\theta_{4}^{-}=0$ and $\theta_{4}^{+}=\pi$. Geometrically, the angle is formed by the asymptote pointing to the direction of $x_{4}$ 's motion and the positive $x_{\|}$axis. See Appendix A for a detailed discussion of the choice of the sign in front of $\arctan G_{4} / L_{4}$.

\subsection{The local and global map, the renormalization and the domain}

\subsubsection{The Poincaré section and the Poincaré map}

Definition 2.6. (The Poincaré section, the local map, the global map and the Poincaré map) We define a section $\left\{x_{4, \|}=-2\right\}$ on the zero-energy level.

- Following the Hamiltonian flow, to the right of this section, we define the local map

$$
\mathbb{L}:\left\{x_{4, \|}=-2, v_{4, \|}>0\right\} \longrightarrow\left\{x_{4, \|}=-2, v_{4, \|}<0\right\},
$$

- and to the left we define the global map

$$
\mathbb{G}:\left\{x_{4, \|}=-2, v_{4, \|}<0\right\} \longrightarrow\left\{x_{4, \|}=-2, v_{4, \|}>0\right\} .
$$

- Finally, we define the Poincaré return map

$$
\mathcal{P}=\mathbb{G} \circ \mathbb{L}:\left\{x_{4, \|}=-2, v_{4, \|}>0\right\} \longrightarrow\left\{x_{4, \|}=-2, v_{4, \|}>0\right\} .
$$

These maps $\mathbb{G}, \mathbb{L}$ and $\mathcal{P}$ are defined by the standard procedure following the Hamiltonian flow. Once we find one orbit going from one section to another, the corresponding map can be defined in a neighborhood of this orbit. The existence of a returning orbit follows from Lemma 2.21. 


\subsubsection{The renormalization map}

Next, we define the renormalization map $\mathcal{R}$, which will be applied after two applications of the Poincaré map. We first fix a large number $\chi \gg 1$, which can be thought as a typical distance between the heavy bodies $Q_{1}$ and $Q_{2}$.

Definition 2.7. We define the renormalization map $\mathcal{R}$ in several steps as follows.

- Given a point $\boldsymbol{x}$, called the base point, on the section $\left\{x_{4, \|}=-2, v_{4, \|}>0\right\}$, we denote by $\mathrm{C}(\boldsymbol{x})$ a cube of size $1 / 2 \sqrt{\lambda \chi}$ centered at $\boldsymbol{x}$, where $\lambda=-2 E_{3}$ is measured at $\boldsymbol{x}$.

Let $\beta=-\arctan \left(x_{1, \perp} / x_{1, \|}\right)$ evaluated at $\boldsymbol{x}$, and denote by $\operatorname{Rot}(\beta)$ the rotation of the plane by angle $\beta$ around the origin.

- We push forward the cube $\mathrm{C}(\boldsymbol{x})$ to the section

$$
\left\{\left(\operatorname{Rot}(\beta)^{-1} \cdot x_{4}\right)_{\|}=\cos \beta x_{4, \|}+\sin \beta x_{4, \perp}=-\frac{2}{\lambda}, v_{4, \|}>0\right\}
$$

along the Hamiltonian flow. We define

$$
\widetilde{\mathbb{G}}:\left\{x_{4, \|}=-2, v_{4, \|}>0\right\} \longrightarrow\left\{\left(\operatorname{Rot}(\beta)^{-1} \cdot x_{4}\right)_{\|}=-\frac{2}{\lambda}, v_{4, \|}>0\right\}
$$

and apply the following procedure to $\widetilde{\mathbb{G}}(\mathrm{C}(\boldsymbol{x}))$.

- (Rotation) We rotate the $x_{\|}$-axis around the origin by angle $\beta$, so that for the center point in each cube, we have that $x_{1, \perp}$ is nearly zero (to be estimated as $\left|x_{1, \perp}\right|=$ $O(\mu / \chi)$, with the error caused by $\widetilde{\mathbb{G}})$. Now, the section $\left\{\left(\operatorname{Rot}(\beta)^{-1} \cdot x_{4}\right)_{\|}=-2 / \lambda, v_{4, \|}>0\right\}$ becomes $\left\{x_{4, \|}=-2 / \lambda, v_{4, \|}>0\right\}$.

- (Rescaling) We zoom in on the configuration space by $\lambda>1$. Simultaneously, we also slow down the velocities by dividing by $\sqrt{\lambda}$. Now, the section $\left\{x_{4, \|}=-2 / \lambda, v_{4, \|}>0\right\}$ becomes $\left\{x_{4, \|}=-2, v_{4, \|}>0\right\}$.

- (Reflection) We reflect the whole system along the $x$-axis.

- Finally, we reset $\chi$ to be equal to the value $\lambda\left|x_{1, \|}\right|$ evaluated at $\boldsymbol{x}$.

We have

$$
\mathcal{R}: \widetilde{\mathbb{G}}(\mathrm{C}(\boldsymbol{x}))\left(\subset\left\{\left(\operatorname{Rot}(-\beta) \cdot x_{4}\right)_{\|}=-\frac{2}{\lambda}, v_{4, \|}>0\right\}\right) \longrightarrow\left\{x_{4, \|}=-2, v_{4, \|}>0\right\},
$$

and

$$
\begin{aligned}
& \mathcal{R}\left(E_{3}, \ell_{3}, e_{3}, g_{3} ; x_{1}, v_{1} ; e_{4}, g_{4}\right)= \\
& \left(\frac{E_{3}}{\lambda}, \ell_{3}, e_{3},-\left(g_{3}-\beta\right) ; \lambda\left[\begin{array}{cc}
1 & 0 \\
0 & -1
\end{array}\right] \operatorname{Rot}(\beta) x_{1},\left[\begin{array}{cc}
1 & 0 \\
0 & -1
\end{array}\right] \frac{\operatorname{Rot}(\beta) v_{1}}{\sqrt{\lambda}} ; e_{4},-\left(g_{4}-\beta\right)\right) .
\end{aligned}
$$

The renormalization also sends time $t$ to $\lambda^{3 / 2} t$ and the Poincaré-Cartan invariant gets multiplied by $\lambda^{1 / 2}$. 
Remark 2.8. The primary goal of the definition of the renormalization map is to rescale the lower ellipse in Figure 2 to the size of the lower ellipse in Figure 1. The reflection is needed since the motions on the two ellipses have opposite orientations (compare the arrows in Figures 1 and 2). The rotation is needed since we want to put $x_{1}$ on the horizontal axis, however, $x_{1}$ has some angular momentum relative to zero, and hence $v_{1}$ forms an angle with $x_{1}$, which moves $x_{1}$ away from the horizontal axis.

We will iterate the map

$$
\mathcal{R} \circ \widetilde{\mathbb{G}} \circ(\mathbb{G} \circ \mathbb{L})^{2}:\left\{x_{4, \|}=-2, v_{4, \|}>0\right\} \longrightarrow\left\{x_{4, \|}=-2, v_{4, \|}>0\right\} .
$$

We shall show that for orbits of interest $\mathcal{R}$ sends $\chi$ to $\lambda \chi(1+O(\mu))$. Thus, $\chi$ will grow to infinity exponentially under iteration. Hence, $\beta=O\left(\chi^{-1 / 2}\right)$ decays exponentially to zero. Without loss of generality, we always assume in our estimates that $1 / \chi \ll \mu$.

\subsection{Asymptotics of the local and global map}

\subsubsection{The standing assumptions}

To simplify the presentation, we list standard assumptions that we will impose on the initial or final values of the local and global map, respectively.

We introduce

$$
\begin{aligned}
& K:= \\
& \sup \max \left\{\left\|d \mathbf{G}_{e_{4}, 1,4}\left(e_{3}, g_{3}, E_{3}\right)\right\|_{\infty}+\left\|\frac{\partial \mathbf{G}_{e_{4}, 1,4}}{\partial e_{4}}\left(e_{3}, g_{3}, E_{3}\right)\right\|_{\infty},\left\|d\left(e_{4}^{\prime}, e_{4}^{\prime \prime}\right)\left(e_{3}, g_{3}\right)\right\|_{\infty}\right\}+1,
\end{aligned}
$$

where the sup is taken over $\dagger=*, * *$, and over all $\left(e_{3}, g_{3}, E_{3}, e_{4}\right)$ in a $\bar{\delta}$-neighborhood of $\left(e_{3}, g_{3}, E_{3}, e_{4}\right)^{\dagger}$, the maps $\mathbf{G}, e_{4}^{\prime}$ and $e_{4}^{\prime \prime}$ are as in Lemma 2.4 , and the $\|\cdot\|_{\infty}$ norm for a linear map $M: \mathbb{R}^{n} \rightarrow \mathbb{R}^{m}$ is defined as $\sup \|M v\|_{\infty}$, where the sup is taken among all $v \in \mathbb{R}^{n}$ with $\|v\|_{\infty}=1$.

We consider $0<\delta<\bar{\delta} / K^{2}$ and fix some large numbers $C_{0}$ and $C_{0}^{\prime}$. For $\hat{\lambda}=1$ or $\lambda_{0}$ as in Lemma 2.4, we use the following standing assumption for the local map.

$\mathbf{A L}(\hat{\lambda}):$

(AL.3) Initially on the section $\left\{x_{4, \|}=-2, v_{4, \|}>0\right\}$ we have

$$
\left\|\left(e_{3}, g_{3}-\sigma(\hat{\lambda}) \cdot \pi, \frac{E_{3}}{\hat{\lambda}}\right)-\left(e_{3}, g_{3}, E_{3}\right)^{\dagger}\right\|_{\infty}<K^{\dagger} \delta
$$

(AL.1) the initial values of $\left(x_{1}, v_{1}\right)$ satisfy

$$
x_{1, \|} \leqslant-\chi, \quad\left|x_{1, \perp}\right| \leqslant C_{0} \mu, \quad\left|v_{1, \perp}\right| \leqslant \frac{C_{0}}{\chi}, \quad 0<-v_{1, \|}<C_{0} ;
$$


(AL.4) the incoming and outgoing asymptotes of the nearly hyperbolic motion of $x_{4}, v_{4}$ satisfy

$$
\left|\theta_{4}^{-}\right| \leqslant C_{0} \mu \quad \text { and } \quad\left|\bar{\theta}_{4}^{+}-\pi\right| \leqslant \tilde{\theta},
$$

and the initial value of $e_{4}$ satisfies $\left|e_{4}-e_{4}^{\dagger}\right|<K^{\dagger} \delta$, where

- $\dagger=*, * *, K^{*}=1$ and $K^{* *}=K$;

- $\tilde{\theta} \ll 1$ is a constant independent of $\chi$ and $\mu$;

- $\sigma:\left\{1, \lambda_{0}\right\} \rightarrow\{0,1\}$ is defined as $\sigma(1)=0$ and $\sigma\left(\lambda_{0}\right)=1$.

We use the following standing assumption for the global map.

$\mathbf{A G}(\hat{\lambda}):$

(AG.3) Initially on the section $\left\{x_{4, \|}=-2, v_{4, \|}<0\right\}$, we have

$$
\left\|\left(e_{3}, g_{3}-\sigma(\hat{\lambda}) \cdot \pi, \frac{E_{3}}{\hat{\lambda}}\right)-\mathbf{G}_{e_{4}^{\dagger}, i, 4}\left(e_{3}, g_{3}, E_{3}\right)^{\dagger}\right\|_{\infty}<K K^{\dagger} \delta
$$

where $\dagger=*, * *$ and $i=1,2$ correspond to the first and second collisions;

(AG.1) the initial conditions of $x_{1}$ and $v_{1}$ satisfy

$$
-1.1 \chi \leqslant x_{1, \|} \leqslant-\chi, \quad\left|x_{1, \perp}\right| \leqslant C_{0}^{\prime} \mu, \quad\left|v_{1, \perp}\right| \leqslant \frac{C_{0}^{\prime}}{\chi}, \quad \frac{1}{C_{0}^{\prime}}<-v_{1, \|}<C_{0}^{\prime}
$$

(AG.4) on the section $\left\{x_{4, \|}=-2\right\}$, we have that $\left|x_{4, \perp}\right|<C_{0}^{\prime}$ holds both at initial and final moments.

If $\hat{\lambda}=1$, we abbreviate $\mathbf{A L}=\mathbf{A L}(1)$ and $\mathbf{A} \mathbf{G}=\mathbf{A} \mathbf{G}(1)$.

We stress that in both $\mathbf{A L}(\hat{\lambda})$ and $\mathbf{A G}(\hat{\lambda})$, we consider only orbits on the zero-energy level and the zeroth total angular momentum level of the Hamiltonian (1.1).

Remark 2.9. - In $\mathbf{A L}(\hat{\lambda})$, we ask the initial values of $\left(x_{3}, v_{3}\right)$ and $\left(x_{4}, v_{4}\right)$ to be close to Gerver's value in Lemma 2.4. The assumption on $\left(x_{1}, v_{1}\right)$ requires $Q_{1}$ to be far away and not to have too much energy. We also require the outgoing asymptote to be almost horizontal, which forces $Q_{3}$ and $Q_{4}$ to have a close encounter, since otherwise $Q_{4}$ moves on a slightly perturbed hyperbola whose outgoing asymptote will not be nearly horizontal.

- In $\mathbf{A G}(\hat{\lambda})$, the main requirement is (AG.4), where we require $\left|x_{4, \perp}\right|$ to be bounded at both the initial and final moments. This will force the motion of $\left(x_{4}, v_{4}\right)$ to be close to a horizontal free motion for most of the time. 


\subsubsection{The asymptotes of the local, global maps}

In the next two lemmas, our notation is such that $\mathbb{L}$ and $\mathbb{G}$ send unbarred variables to barred variables.

The next lemma shows that the real local map $\mathbb{L}$ is well approximated by the Gerver map $\mathbf{G}$ in the $\mathscr{C}^{0}$ sense. Its proof will be given in $\S 10.1$.

LEMma 2.10. Assume $\mathbf{A L}(\hat{\lambda})$, with $\hat{\lambda}=1$ or $\hat{\lambda}=\lambda_{0}$. Then, after the application of $\mathbb{L}$, the following asymptotics hold uniformly:

$$
\left(\bar{E}_{3}, \bar{e}_{3}, \bar{g}_{3}\right)=\mathbf{G}_{e_{4}}\left(E_{3}, e_{3}, g_{3}\right)+o(1)
$$

as $1 / \chi \ll \mu \rightarrow 0$ and $\tilde{\theta} \rightarrow 0$.

The next lemma deals with the $\mathscr{C}^{0}$ estimates for the global map $\mathbb{G}$.

Lemma 2.11. Assume $\mathbf{A G}(\hat{\lambda})$ with $\hat{\lambda}=1$ or $\hat{\lambda} \lambda_{0}$. Then, there exist constants $C_{3}$ and $C_{4}$ such that, after the application of $\mathbb{G}$ and $\widetilde{\mathbb{G}} \circ \mathbb{G}$, the following estimates hold uniformly in $\chi$ and $\mu$ as $1 / \chi \ll \mu \rightarrow 0$ :

(a) $\left|\bar{E}_{3} / E_{3}-1\right| \leqslant C_{3} \mu,\left|\bar{G}_{3} / G_{3}-1\right| \leqslant C_{3} \mu,\left|\bar{g}_{3}-g_{3}\right| \leqslant C_{3} \mu$;

(b) $\left|\theta_{4}^{+}-\pi\right| \leqslant C_{4} \mu,\left|\bar{\theta}_{4}^{-}\right| \leqslant C_{4} \mu$;

(c) the return times defining $\mathbb{G}$ and $\widetilde{\mathbb{G}} \circ \mathbb{G}$ are bounded by $3 \chi$.

The proof of this lemma is given in $\S 6.5$. From now on, we choose the constant $C_{0}$ in $\mathbf{A L}$ to be larger than $C_{4}$ in Lemma 2.11 .

\subsubsection{Dynamics of $\left(x_{1}, v_{1}\right)$ under the renormalized Poincaré map}

The next lemma deals with the $\mathscr{C}^{0}$ estimates of $\left(x_{1}, v_{1}\right)$. The proof is also in $\S 6.5$.

Lemma 2.12. Fix $\hat{\lambda}=1$. There exist constants $C_{0}, C_{0}^{\prime}, c_{1}, \bar{c}_{1}, C_{1}>0$, with $\bar{c}_{1}<C_{0}$, such that the following holds. Consider an orbit with initial condition $\boldsymbol{x}$ satisfying

(i) (AL.3) and (AL.4) are satisfied when applying $\mathbb{L}$ for the first time, and (AG.4) is satisfied when applying $\mathbb{G}$ for the first time;

(ii) initially on the section $\left\{x_{4, \|}=-2, v_{4, \|}>0\right\}$,

$$
G_{0}=0, \quad-\chi-\frac{1}{\sqrt{\chi}} \leqslant x_{1, \|}(0) \leqslant-\chi, \quad\left|x_{1, \perp}(0)\right| \leqslant \frac{1}{\sqrt{\chi}}, \quad-\bar{c}_{1} \leqslant v_{1, \|}(0) \leqslant-c_{1} .
$$

Then, we have

(a) after the application of $\mathcal{P},(\mathrm{AL} .1)$ is satisfied for $\left(x_{1}, v_{1}\right)$;

(b) after the application of $\mathbb{L}$ and $\mathbb{L} \circ \mathcal{P}$ (whenever the second $\mathbb{L}$ is defined), (AG.1) is satisfied for $\left(x_{1}, v_{1}\right)$. 
Assume (i' and (ii) in place of (i) and (ii) above, where

$\left(\mathrm{i}^{\prime}\right)$ (AL.3) and (AL.4) are satisfied when applying $\mathbb{L}$ for both the first and the second times, and (AG.4) is satisfied when applying $\mathbb{G}$ for both the first and the second times.

Then, we have

(c) after the application of $\mathcal{R} \circ \widetilde{\mathbb{G}} \circ \mathcal{P}^{2}$, where $\mathcal{R}$ is based at the point $\mathcal{P}^{2}(\boldsymbol{x})$, we get that the renormalized $\chi$, denoted by $\tilde{\chi}$, satisfies $\lambda\left(1+C_{1}^{-1} \mu\right) \chi \leqslant \tilde{\chi} \leqslant \lambda\left(1+C_{1} \mu\right) \chi$, and the renormalized orbit parameters $G_{0}, x_{1}$ and $v_{1}$ satisfy $(2.8)$, with $\chi$ replaced by $\tilde{\chi}$.

Remark 2.13. We explain the physical meaning of the lemma. The assumption implies that both $v_{4}$ and $v_{3}$ are of order 1 . By (2.8), $v_{1}$ is also of order 1 and $v_{1, \perp}$ is bounded by $C / \chi$. In Remark 2.2 we have stressed that $\mu v_{1}$ instead of $v_{1}$ is close to the velocity of $Q_{1}$. So $Q_{1}$ moves to the left with a velocity of order $\mu$ having a tiny vertical component. It takes $Q_{4}$ a long time of order $\chi$ to complete a return and during this time, $Q_{1}$ moves a distance of order $\mu \chi$. This gives the estimates of $x_{4, \|}$ and $\tilde{\chi}$ after renormalization. The energy exchange between $Q_{1}$ and $Q_{4}$ will change $v_{4, \|}$ significantly, but the renormalization will slow down $v_{4, \|}$ to the interval $\left[-\bar{c}_{1},-c_{1}\right]$. The rotation in the renormalization controls $x_{4, \perp}$.

\subsection{The tangent dynamics and the strong expansion}

Definition 2.14. Given $\delta<\bar{\delta} / K^{2}$, where $\bar{\delta}$ is in Lemma 2.4, we define the following open sets in the section $\left\{x_{4, \|}=-2, v_{4, \|}>0\right\}$ on the zero-energy level by

$$
\begin{aligned}
& U_{1}(\delta)=\left\{\mathbf{A L} \text {, except the } \bar{\theta}_{4}^{+} \text {assumption therein, holds with } \dagger=*\right\}, \\
& U_{2}(\delta)=\left\{\mathbf{A L}, \text { except the } \bar{\theta}_{4}^{+} \text {assumption therein, holds with } \dagger=* *\right\}, \\
& U_{0}(\delta)=\left\{\mathbf{A L}\left(\lambda_{0}\right), \text { except the } \bar{\theta}_{4}^{+} \text {assumption therein, holds with } \dagger=*\right\} .
\end{aligned}
$$

Remark 2.15. (1) The sets $U_{j}(\delta), j=1,2$, are neighborhoods of Gerver's collision points in Lemma 2.4. The set $U_{0}$ is introduced to study the dynamics without the renormalization.

(2) In the definition we do not restrict $\ell_{3}$, since $\ell_{3}$ can take any value in $[0,2 \pi)$. We do not restrict $v_{1, \perp}$, since it can be bounded by $C / \chi$ by the information in (2.8). We also get rid of the assumption on the final value $\bar{\theta}_{4}^{+}$in $\mathbf{A L}$.

\subsubsection{The invariant cone fields}

We introduce the following cone fields. 
Definition 2.16. (Cone fields) Let

(1) $\mathcal{K}_{1} \subset T_{U_{1}(\delta)}\left(\mathbb{R}^{7} \times \mathbb{T}^{3}\right)$ be the set of vectors forming an angle less than a small number $\eta$ with $\operatorname{span}\left(d \mathcal{R} w_{2}, d \mathcal{R} \widetilde{w}\right)$,

(2) $\mathcal{K}_{0} \subset T_{U_{0}(\delta)}\left(\mathbb{R}^{7} \times \mathbb{T}^{3}\right)$ be the set of vectors forming an angle less than a small number $\eta$ with $\operatorname{span}\left(w_{2}, \widetilde{w}\right)$,

(3) $\mathcal{K}_{2} \subset T_{U_{2}(\delta)}\left(\mathbb{R}^{7} \times \mathbb{T}^{3}\right)$ be the set of vectors forming an angle less than $\eta$ with $\operatorname{span}\left(w_{1}, \widetilde{w}\right)$,

where

$$
\widetilde{w}=\frac{\partial}{\partial \ell_{3}} \text { and } w_{j}=\left(\frac{\sqrt{e_{4}^{2}-1}}{L_{3} e_{4}} \frac{\partial}{\partial e_{4}}-\frac{1}{L_{3} e_{4}^{2}} \frac{\partial}{\partial g_{4}}\right), \quad j=1,2 .
$$

We choose our parameters to be $0<1 / \chi \ll \mu \ll \delta \ll \eta \ll 1$.

The next lemma establishes the (partial) hyperbolicity of the Poincaré map.

Lemma 2.17. There exists a constant $c$ such that, for all $\boldsymbol{x} \in U_{1}(\delta)$ satisfying $\mathcal{P}(\boldsymbol{x}) \in$ $U_{2}(\delta)$, and for all $\boldsymbol{x} \in U_{2}(\delta)$ satisfying $\mathcal{P}(\boldsymbol{x}) \in U_{0}(\delta)$, we have

(a) (Invariance) $d \mathcal{P}\left(\mathcal{K}_{1}\right) \subset \mathcal{K}_{2}, d \mathcal{P}\left(\mathcal{K}_{2}\right) \subset \mathcal{K}_{0}$ and $d(\mathcal{R} \circ \widetilde{G} \circ \mathcal{P})\left(\mathcal{K}_{2}\right) \subset \mathcal{K}_{1}$, where the base point defining $\mathcal{R}$ can be chosen to be any point in $U_{0}(\delta)$, since $\delta \ll \eta$.

(b) (Expansion) If $v \in \mathcal{K}_{1}$, then $\|d \mathcal{P}(v)\| \geqslant c \chi\|v\|$. If $v \in \mathcal{K}_{2}$, then $\|d \mathcal{P}(v)\| \geqslant c \chi\|v\|$ and $\|d(\mathcal{R} \circ \widetilde{G} \circ \mathcal{P})(v)\| \geqslant c \chi\|v\|$.

We give the proof in $\S 3$. The next lemma follows directly from Definition 2.16.

Lemma 2.18. (a) The vector $\widetilde{w}=\partial / \partial \ell_{3}$ is in $\mathcal{K}_{i}$.

(b) For any plane $\Pi$ in $\mathcal{K}_{i}$ the projection map

$$
\pi_{e_{4}, \ell_{3}}=\left(d e_{4}, d \ell_{3}\right): \Pi \longrightarrow \mathbb{R}^{2}
$$

is one-to-one.

\subsubsection{The admissible surfaces}

Definition 2.19. (Admissible surfaces) We call a 2-dimensional $\mathscr{C}^{1}$ surface $S \subset U_{i}(\delta)$ admissible if $T S \subset \mathcal{K}_{i}, i=0,1,2$.

Since Poincare maps send admissible surfaces to admissible surfaces if the images lie in $U_{j}(\delta), j=1,2$, by Lemmas 2.17 and 2.18 , we can restrict the Poincaré maps to admissible surfaces to obtain 2-dimensional maps. The reduction is done as follows. We introduce two cylinder sets

$$
\mathcal{C}_{0}(\delta)=\mathcal{C}_{1}(\delta):=\left(e_{4}^{*}-\delta, e_{4}^{*}+\delta\right) \times \mathbb{T}^{1} \quad \text { and } \quad \mathcal{C}_{2}(\delta)=\left(e_{4}^{* *}-K \delta, e_{4}^{* *}+K \delta\right) \times \mathbb{T}^{1} .
$$


By Lemma 2.18, we get that each piece of admissible surface $S \subset U_{i}(\delta)$ is the graph of a function $\mathcal{S}$ defined on $\mathcal{C}_{i}(\delta), i=0,1,2$. So we get that $\mathcal{P} \circ \mathcal{S}$ is a function of two variables $\left(e_{4}, \ell_{3}\right)$. However, for most points in $\mathcal{C}_{i}(\delta)$, the map $\mathcal{P} \circ \mathcal{S}$ is not defined, since the orbit might not return.

Given a piece of admissible surface $S \subset U_{j}(\delta)$, we next introduce the maps $\mathcal{Q}_{1}, \mathcal{Q}_{2}$ and $\mathcal{Q}_{0}$ from a subset of $\mathcal{C}_{1}(\delta)$ to $\mathcal{C}_{2}(\delta)$, a subset of $\mathcal{C}_{2}(\delta)$ to $\mathcal{C}_{0}(\delta)$ and a subset of $\mathcal{C}_{0}(\delta)$ to $\mathcal{C}_{2}(\delta)$, respectively:

$$
\mathcal{Q}_{1}:=\pi_{e_{4}, \ell_{3}} \mathcal{P}(\mathcal{S}(\cdot, \cdot)), \quad \mathcal{Q}_{2}:=\pi_{e_{4}, \ell_{3}} \mathcal{P}(\mathcal{S}(\cdot, \cdot)), \quad \mathcal{Q}_{0}:=\pi_{e_{4}, \ell_{3}} \mathcal{P} \mathcal{R} \widetilde{\mathbb{G}}(\mathcal{S}(\cdot, \cdot)) .
$$

where the base point of $\mathcal{R}$ in $\mathcal{Q}_{0}$ will be specified below. The domain of $\mathcal{Q}_{1}$ can be found by taking $\mathcal{Q}_{1}^{-1}\left(\mathcal{C}_{2}(\delta)\right) \cap \mathcal{C}_{1}(\delta)$, and similarly for $\mathcal{Q}_{2}$ and $\mathcal{Q}_{0}$. This completes the reduction of the Poincaré maps to 2-dimensional maps.

Definition 2.20. (Essential admissible surfaces) For $\delta^{\prime}<\delta$, we call an admissible surface $S \subset U_{j}(\delta) \delta^{\prime}$-essential if $\pi_{e_{4}, \ell_{3}} S$ contains $\mathcal{C}_{j}\left(\delta^{\prime}\right), j=1,2$.

LEMma 2.21. Given $0<\delta^{\prime}<\delta \leqslant \bar{\delta} / K^{2}$, we have the following for $\chi$ sufficiently large.

(a) Given a $\delta^{\prime}$-essential admissible surface $S \subset U_{1}(\delta)$ and

$$
\tilde{e}_{4} \in\left(e_{4}^{*}-\delta^{\prime}+\frac{1}{\chi}, e_{4}^{*}+\delta^{\prime}-\frac{1}{\chi}\right),
$$

there exists $\tilde{\ell}_{3}$ such that $\pi_{e_{4}} \mathcal{P S}\left(\tilde{e}_{4}, \tilde{\ell}_{3}\right)=e_{4}^{* *}$. Moreover, there exists a neighborhood $V_{1}\left(\tilde{e}_{4}\right)\left(\subset \mathcal{C}_{1}\left(\delta^{\prime}\right)\right)$ of $\left(\tilde{e}_{4}, \tilde{\ell}_{3}\right)$ of diameter $O(1 / \chi)$ such that $\mathcal{Q}_{1}$ maps $V_{1}\left(\tilde{e}_{4}\right)$ surjectively to $\mathcal{C}_{2}(\delta)$

(b) Given a $\delta^{\prime}$-essential admissible surface $S \subset U_{2}(\delta)$ and

$$
\tilde{e}_{4} \in\left(e_{4}^{* *}-K \delta^{\prime}+\frac{1}{\chi}, e_{4}^{* *}+K \delta^{\prime}-\frac{1}{\chi}\right),
$$

there exists $\tilde{\ell}_{3}$ such that $\pi_{e_{4}} \mathcal{P} \mathcal{S}\left(\tilde{e}_{4}, \tilde{\ell}_{3}\right)=e_{4}^{*}$. Also, there is a neighborhood $V_{2}\left(\tilde{e}_{4}\right)\left(\subset \mathcal{C}_{2}\left(\delta^{\prime}\right)\right)$ of $\left(\tilde{e}_{4}, \tilde{\ell}_{3}\right)$ of diameter $O(1 / \chi)$ such that $\mathcal{Q}_{2}$ maps $V_{2}\left(\tilde{e}_{4}\right)$ surjectively to $\mathcal{C}_{0}(\delta)$.

(c) Given a $\delta^{\prime}$-essential admissible surface $S \subset U_{0}(\delta)$ and

$$
\tilde{e}_{4} \in\left(e_{4}^{*}-\delta^{\prime}+\frac{1}{\chi}, e_{4}^{*}+\delta^{\prime}-\frac{1}{\chi}\right)
$$

there exists $\tilde{\ell}_{3}$ such that $\pi_{e_{4}} \mathcal{P S}\left(\tilde{e}_{4}, \tilde{\ell}_{3}\right)=e_{4}^{* *}$. Moreover, defining the renormalization $\mathcal{R}$ based at the point $\mathcal{S}\left(\tilde{e}_{4}, \tilde{\ell}_{3}\right)$, there exists a neighborhood $V_{0}\left(\tilde{e}_{4}\right)\left(\subset \mathcal{C}_{0}\left(\delta^{\prime}\right)\right)$ of $\left(\tilde{e}_{4}, \tilde{\ell}_{3}\right)$ of diameter $O(1 / \chi)$ such that $\mathcal{Q}_{0}$ maps $V_{0}\left(\tilde{e}_{4}\right)$ surjectively to $\mathcal{C}_{2}(\delta)$.

(d) For points in $V_{i}\left(\tilde{e}_{4}\right)$ from parts (a) and (b) $(i=0,1,2)$, there exist constants $c$, $\mu_{0}$ and $\chi_{0}$ such that, for $\mu<\mu_{0}$ and $\chi>\chi_{0}$, we have that the particles $Q_{3}$ and $Q_{4}$ avoid collisions before the next return, and the minimal distance $d$ between $Q_{3}$ and $Q_{4}$ satisfies $c \mu \leqslant d \leqslant \mu / c$. Moreover, $Q_{1}$ and $Q_{4}$ do not collide. 
Parts (a)-(c) of the lemma are proved in $\S 6.6$. Part (d) is given in $\S 6.4$ as well as Lemma $10.2(\mathrm{~b})$.

\subsection{Proof of Theorem 1}

Step 1. (Concatenating Lemmas 2.10-2.12.)

We will iterate $\mathcal{R} \circ \widetilde{\mathbb{G}} \circ \mathbb{G} \circ \mathbb{L} \circ \mathbb{G} \circ \mathbb{L}$. Suppose we have a point $\boldsymbol{x} \in U_{1}(\delta)$ whose images $\mathcal{P}(\boldsymbol{x}) \in U_{2}(\delta)$ and $\mathcal{R} \widetilde{\mathbb{G}} \mathcal{P}^{2}(\boldsymbol{x}) \in U_{1}(\delta)$, where $\mathcal{R}$ is defined with the base point $\mathcal{P}^{2}(\boldsymbol{x})$. We assume in addition that $(2.8)$ is satisfied for $\boldsymbol{x}$. Leaving the existence of such a point to be addressed later, we first show how the assumptions of Lemmas 2.10-2.12 are satisfied.

The assumption AL (except the $\theta_{4}^{+}$assumption therein) for Lemma 2.10 is satisfied since $\boldsymbol{x} \in U_{1}(\delta)$. To proceed, we pick some small $\tilde{\theta}$ and assume that $\left|\bar{\theta}_{4}^{+}-\pi\right|<\tilde{\theta}$ in (AL.4) is satisfied.

The conclusion of Lemma 2.10 combined with Lemma 2.4 implies (AG.3) by choosing $\mu$ and $\tilde{\theta}$ sufficiently small, and the assumption that $\boldsymbol{x} \in U_{1}(\delta)$ and $\mathcal{P}(\boldsymbol{x}) \in U_{2}(\delta)$ implies (AG.4). Next, the assumptions of Lemma 2.12 for the first application of $\mathcal{P}$ are satisfied, so we get (AG.1). Now, the assumption AG is satisfied.

Now, we apply Lemma 2.11 to conclude that $E_{3}, G_{3}$ and $g_{3}$ have $O(\mu)$-oscillations and the initial and final angles of asymptotes are $O(\mu)$ close to zero and $\pi$, respectively. By choosing $\mu$ small, we see that the $\theta_{4}^{+}$assumption in (AL.4) is automatically satisfied. That is to say, if $\mathbb{G}$ is applicable after the application of $\mathbb{L}$, then the $\theta_{4}^{+}$assumption in (AL.4) is redundant.

Next, we consider the second application of $\mathbb{L}$. In the first application of $\mathbb{L}$, we see that $\mathbb{L}$ is approximated by $\mathbf{G}$ by Lemma 2.4. Next, the application of $\mathbb{G}$ gives only a $\mu$-oscillation to the values of $E_{3}, g_{3}$ and $e_{3}$, so applying Lemma 2.4, we see that (AL.3) is satisfied for the second application of $\mathbb{L}$. The $\theta_{4}^{-}$and $e_{4}$ parts of (AL.4) are satisfied since we have $\mathcal{P}(\boldsymbol{x}) \in U_{2}(\delta)$. Lemma 2.12 implies that (AL.1) is satisfied. The only missing assumption in (AL.4) is the assumption on the outgoing angle of asymptote $\bar{\theta}_{4}^{+}$, which is again redundant under the assumption $\mathcal{R} \widetilde{\mathbb{G}} \mathcal{P}^{2}(\boldsymbol{x}) \in U_{1}(\delta)$.

We can now apply Lemma 2.10 for the second time. Similarly, we verify the assumption for the second application of $\mathbb{G}$. After $\mathcal{R} \widetilde{\mathbb{G}} \mathcal{P}^{2}$, the assumption (AL.1) and (2.8) are provided by part (c) of Lemma 2.12. The assumption (AL.3) and $e_{4}$ part of (AL.4) is provided by Lemma 2.4 and the renormalization applied to $E_{3}$. The assumptions on the angles of asymptotes in (AL.4) are again given by the existence of returning orbits, to be addressed below. So we can apply Lemma 2.10 for the third time. 
Step 2. (Choosing the initial piece of admissible surface.)

We choose a number $\delta^{\prime}<\delta / K^{2}$. Then, by Definitions 2.16 and 2.19 , on $S_{0}$ we have

$$
\left|E_{3}-E_{3}^{*}\right|<\delta^{\prime}+\eta \delta, \quad\left|e_{3}-e_{3}^{*}\right|<\delta^{\prime}+\eta \delta \quad \text { and } \quad\left|g_{3}-g_{3}^{*}\right|<\delta^{\prime}+\eta \delta,
$$

where $\eta$ is the small number in Definition 2.16. Here, we choose $\eta$ so small that $\delta^{\prime}+\eta \delta<\delta$. Such a piece of $\delta^{\prime}$-essential admissible surface $S_{0}$ exists by explicit construction as follows. We first take an integral curve in $U_{1}\left(\delta^{\prime}\right)$ along the vector field $w_{1}$ in Definition 2.16 such that its $e_{4}$ component is the interval $\left(e_{4}^{*}-\delta^{\prime}, e_{4}^{*}+\delta^{\prime}\right)$. Then the surface $S_{0}$ can be chosen as the product of the curve with $\mathbb{T}^{1}\left(\ni \ell_{3}\right)$.

Step 3. (Non-collision singularities.)

We wish to construct a singular orbit with initial value in $S_{0}$. We define $S_{i}$ inductively so that $S_{1}$ is a $\delta^{\prime}$-essential component of $\mathcal{P}\left(S_{0}\right) \cap U_{2}(\delta)$, and, for $i \geqslant 2, S_{i}$ is a $\delta^{\prime}$-essential component of $(\mathcal{P} \mathcal{R} \widetilde{\mathbb{G}} \mathcal{P})\left(S_{i-1}\right) \cap U_{2}(\delta)$ (we shall show below that such components exist). Given a $\delta^{\prime}$-essential admissible surface $S_{i} \subset U_{2}(\delta)$, choose

$$
\tilde{e}_{4} \in\left(e_{4}^{* *}-K \delta^{\prime}+\frac{1}{\chi}, e_{4}^{* *}+K \delta^{\prime}-\frac{1}{\chi}\right) .
$$

Then, the hypothesis of Lemma 2.21 (b) is satisfied, so there exist $\tilde{\ell}_{3}$ and $V_{2, i}\left(\tilde{e}_{4}\right)$ satisfying Lemma $2.21(\mathrm{~b})$. In particular, $V_{2, i}\left(\tilde{e}_{4}\right)$ is a subset of $\mathcal{C}_{2}\left(\delta^{\prime}\right)$ with diameter $O(\mu / \chi)$, and $\left(\tilde{e}_{4}, \tilde{\ell}_{3}\right) \in V_{2, i}\left(\tilde{e}_{4}\right)$. It follows that, for every $\left(e_{4}, \ell_{3}\right) \in V_{2, i}\left(\tilde{e}_{4}\right)$, we have

$$
e_{4} \in\left(e_{4}^{* *}-K \delta^{\prime}, e_{4}^{* *}+K \delta^{\prime}\right)
$$

In fact, this is true for every $\left(e_{4}, \ell_{3}\right)$ in $\bar{V}_{2, i}\left(\tilde{e}_{4}\right)$, the closure of $V_{2, i}\left(\tilde{e}_{4}\right)$. Therefore, $\bar{V}_{2, i}\left(\tilde{e}_{4}\right) \subset \mathcal{C}_{2}\left(\delta^{\prime}\right)$, and $\mathcal{S}_{i}$ is defined on $\bar{V}_{2, i}\left(\tilde{e}_{4}\right)$. Let $\widehat{S}_{i}=\mathcal{S}_{i}\left(\bar{V}_{2, i}\left(\tilde{e}_{4}\right)\right)$. Then, because $\mathcal{S}_{i}$ is a continuous bijection, $\widehat{S}_{i}$ is closed. Also, because $S_{i}=\mathcal{S}_{i}\left(\mathcal{C}_{2}\left(\delta^{\prime}\right)\right)$, we have $\widehat{S}_{i} \subset S_{i}$. Likewise, $(\mathcal{P} \mathcal{R} \widetilde{\mathbb{G}} \mathcal{P})^{-1}\left(\widehat{S}_{i}\right)$ is a closed subset of $(\mathcal{P} \mathcal{R} \widetilde{\mathbb{G}} \mathcal{P})^{-1}\left(S_{i}\right)$. We shall show below that $(\mathcal{P} \mathcal{R} \widetilde{\mathbb{G}} \mathcal{P})^{-1}\left(S_{i+1}\right) \subset \widehat{S}_{i}$. It follows by induction on $i$ that

$$
\left\{\mathcal{P}^{-1}(\mathcal{P} \mathcal{R} \widetilde{G} \mathcal{P})^{-i} \widehat{S}_{i+1}\right\}_{i=0}^{\infty}
$$

is a family of nested non-empty sets, whose intersection $X$ is therefore non-empty. Choose any $\boldsymbol{x} \in X$. (In fact, $X$ has only one element, but we do not need to use that fact.) We claim that $\boldsymbol{x}$ has a singular orbit. We define $t_{i}$ as the time of the orbit's $(2 i)$-th visit to the section $\left\{x_{4, \|}=-2, x_{4, \|}>0\right\}$. By Lemmas 2.4 and 2.10, the rescaled energy is close to Gerver's values in Lemma 2.4 and the rescaling factor satisfies $\lambda_{0}+\tilde{\delta} \geqslant \lambda \geqslant \lambda_{0}-\tilde{\delta}>1$, where $\lambda_{0}$ is in Lemma 2.4 and $\tilde{\delta}=\delta^{\prime}+\eta \delta$, so the unrescaled energy of $\left(x_{3}, v_{3}\right)$ satisfies

$$
\frac{1}{2}\left(\lambda_{0}-\tilde{\delta}\right)^{i-1} \leqslant-E_{3}\left(t_{i}\right) \leqslant \frac{1}{2}\left(\lambda_{0}+\tilde{\delta}\right)^{i-1} .
$$


According to Lemmas 2.11 and 2.12, and to the total energy conservation (see Lemma 4.5 below for the Hamiltonian), we get that the velocity $\left|v_{4}\right|$ during the $i$ th iteration is bounded from below by

$$
c \sqrt{-E_{3}\left(t_{i}\right)} \geqslant c\left(\lambda_{0}-\tilde{\delta}\right)^{(i-1) / 2} .
$$

Note that in Step 1 the initial conditions for $x_{1}$ and , $v_{1}$ are chosen to satisfy the assumption (2.8). Lemma 2.12 then shows that the assumptions on $x_{1}$ and $v_{1}$ are always satisfied. Thus, we can iterate Lemma 2.12 for arbitrarily many steps.

Now, let us look at the orbit in the physical space without doing any renormalization. Inductively, we have

$$
x_{1, \|}\left(t_{i}\right) \in\left[-\left(1+\mu C_{1}\right)^{i-1 / 2} \chi_{0},-\left(1+\mu C_{1}^{-1}\right)^{i-1 / 2} \chi_{0}\right]
$$

after the $i$ th iteration using Lemma $2.12(\mathrm{c})$, where $\chi_{0}$ is the initial value for $\chi$. Therefore, $x_{1, \|} \rightarrow-\infty$ as $n \rightarrow \infty$. The value of $\chi$ used during each step of $\mathcal{P} \mathcal{R} \widetilde{\mathbb{G}} \mathcal{P}$, denoted by $\chi_{i}\left(=\frac{1}{2} x_{1, \|}\left(t_{i}\right) / E_{3}\left(t_{i}\right)\right)$, is estimated as

$$
\left(\lambda_{0}-\tilde{\delta}\right)^{i-1}\left(1+\mu C_{1}^{-1}\right)^{i-1 / 2} \chi_{0} \leqslant \chi_{i} \leqslant\left(\lambda_{0}+\tilde{\delta}\right)^{i-1}\left(1+\mu C_{1}\right)^{i-1 / 2} \chi_{0} .
$$

Next, for each application of $\mathbb{L}$, the total time is bounded by a uniform constant. For each application of $\mathbb{G}$, the return time is bounded by $3 \chi_{i}$ by Lemma 2.11 (c). So, without the renormalization, the time difference

$$
\left|t_{i+1}-t_{i}\right| \leqslant C\left(\lambda_{0}-\tilde{\delta}\right)^{-3 i / 2} \cdot\left(\lambda_{0}+\tilde{\delta}\right)^{i}\left(1+\mu C_{1}\right)^{i} \chi_{0}
$$

where the constant $C$ absorbs finite powers of $\left(\lambda_{0} \pm \tilde{\delta}\right)$ and $\left(1+\mu C_{1}\right)$, so the total time $t_{*}=\lim _{i \rightarrow \infty} t_{i}$ is bounded as needed. This shows that infinitely many steps complete within finite time, and $x_{1}$ goes to infinity. Since $\mu$ is small and in $U_{j}(\delta), j=1,2$, both $x_{3}$ and $x_{4}$ are bounded, from (2.3) we see that $q_{1}$ also goes to infinity. This implies that both $Q_{1}$ and $Q_{2}$ escape to infinity, since $q_{1}=Q_{1}-Q_{2}$ and the mass center is fixed. We also have that $Q_{3}$ escapes to infinity, since $Q_{3}$ is always close to $Q_{2}$, i.e. $q_{3}$ is bounded. Finally, $Q_{4}$ travels between $Q_{1}$ and $Q_{2}$. To see that no collision occurs during the whole process, we only examine the $Q_{3}-Q_{4}$ and $Q_{1}-Q_{4}$ close encounters whose collisions are excluded by part (d) of Lemma 2.21 (see $\S 6.4$ for more details).

The symbolic dynamics in the statement of the main theorem is due to the fact that we can switch the roles of $Q_{3}$ and $Q_{4}$ after their close encounter. For elastic collisions, such a switch is done by replacing $\alpha$ by $\pi-\alpha$ in (2.5). Both cases ( $\alpha$ and $\pi-\alpha$ ) can be shadowed by Kepler hyperbolic motion when $\mu>0$. See [G3] for more details. In the above, we have been fixing the discrete parameter $\omega=4$ in the definition of Gerver's map 
$\mathbf{G}_{e_{4}, j, \omega}$, i.e. we have been choosing $Q_{4}$ as the traveler. In this case, the global map $\mathbb{G}$ sends points in $U_{j}(\delta)$ to points in $U_{3-j}(\delta), j=1,2$, so we see that $Q_{4}$ winds around $Q_{1}$ once in the sense of Definition 1.1. In general it is the traveler $Q_{\omega}$ that winds around $Q_{1}$.

Step 4. (The induction steps.)

It remains to show that we can find a $\delta^{\prime}$-essential component of $S_{i}$ inside $U_{2}(\delta)$. We proceed inductively, and assume that $S_{i} \subset U_{2}(\delta)$, for $i \geqslant 2$, is a $\delta^{\prime}$-essential admissible surface after application of $(\mathcal{P} \mathcal{R} \widetilde{\mathbb{G}} \mathcal{P})^{i-1}$ to a subset of $S_{1}$. The fact that the $\delta^{\prime}$-essential admissible surface $S_{1}$ exists follows from Lemma 2.21 (a) applied to $S_{0}$. Indeed,

$$
S_{1}=\mathcal{P S}\left(V_{1}\left(\tilde{e}_{4}\right)\right)
$$

We next apply Lemma $2.21(\mathrm{~b})$. For given $\tilde{e}_{4} \in\left(e_{4}^{* *}-K \delta^{\prime}+1 / \chi, e_{4}^{* *}+K \delta^{\prime}-1 / \chi\right)$, there exists $\tilde{\ell}_{3} \in \mathbb{T}^{1}$ such that $\pi_{e_{4}} \mathcal{P}\left(\mathcal{S}_{i}\left(\tilde{e}_{4}, \tilde{\ell}_{3}\right)\right)=e_{4}^{*}$. Moreover, there exists a neighborhood $V_{2, i}\left(\tilde{e}_{4}\right)$ of $\left(\tilde{e}_{4}, \tilde{\ell}_{3}\right)$ such that $\mathcal{Q}_{2}$ maps $V_{2, i}\left(\tilde{e}_{4}\right)$ surjectively onto $\mathcal{C}_{0}\left(\delta^{\prime}\right)$. We denote by $S_{i+1 / 2}$ the image $\mathcal{P}\left(\mathcal{S}_{i}\left(V_{2, i}\left(\tilde{e}_{4}\right)\right)\right)$, which is admissible by Lemma 2.18 and $\delta^{\prime}$-essential. Moreover, every point $\boldsymbol{x} \in S_{i+1 / 2}$ satisfies (AL.3) by Lemma 2.10 and Lemma 2.11 (a), (AL.1) by Lemma 2.12 and (AL.4) by Lemma 2.11 (b) and the $\delta^{\prime}$-essentiality implies that $\pi_{e_{4}} S_{i+1 / 2}=\left(e_{4}^{*}-\delta^{\prime}, e_{4}^{*}+\delta^{\prime}\right)$; thus, $S_{i+1 / 2} \subset U_{0}(\delta)$.

We next apply Lemma 2.21 (c) to find a point $\left(e^{*}, \tilde{\tilde{\ell}}_{3}\right)$ such that

$$
\pi_{e_{4}} \mathcal{P} \mathcal{S}_{i+1 / 2}\left(e_{4}^{*}, \tilde{\tilde{\ell}}_{3}\right)=e_{4}^{* *} .
$$

We now introduce the renormalization $\mathcal{R}$ based at the point $\mathcal{S}_{i+1 / 2}\left(e_{4}^{*}, \tilde{\tilde{\ell}}_{3}\right)$ and define $\mathcal{Q}_{0}$. Again by Lemma $2.21(\mathrm{c})$, there exists a neighborhood $V_{0, i+1 / 2}\left(e_{4}^{*}\right) \subset \mathcal{C}_{0}(\delta)$ such that $\mathcal{Q}_{0}$ maps $V_{0, i+1 / 2}\left(e_{4}^{*}\right)$ surjectively onto $\mathcal{C}_{2}\left(\delta^{\prime}\right)$, and we have that the diameter of $V_{0, i+1 / 2}\left(e_{4}^{*}\right)$ is $O(\mu / \chi)$, which is much smaller than $1 / \sqrt{\chi}$, the size of the domain of $\mathcal{R}$, so $\mathcal{R}$ is well defined on $\widetilde{\mathbb{G}}\left(V_{0, i+1 / 2}\left(e_{4}^{*}\right)\right)$ ( $d \widetilde{\mathbb{G}}$ is bounded).

We simply define

$$
S_{i+1}:=\left\{\boldsymbol{x} \in \mathcal{P} \mathcal{R} \widetilde{\mathbb{G}}\left(\mathcal{S}_{i+1 / 2}\left(V_{0, i+1 / 2}\left(e_{4}^{*}\right)\right)\right): \pi_{e_{4}, \ell_{3}} \boldsymbol{x} \in \mathcal{C}_{2}\left(\delta^{\prime}\right)\right\} .
$$

By Lemma 2.17 (a), we know that $S_{i+1}$ is admissible.

By Definition 2.20, we know that $S_{i+1}$ is $\delta^{\prime}$-essential. On $S_{i+1}$, we always have (2.9) for the variables $E_{3}, e_{3}$ and $g_{3}$ by Definitions 2.16 and 2.19. The variables $\left(x_{1}, v_{1}\right)$ are always controlled by part (c) of Lemma 2.12, and the angle $g_{4}$ is controlled by part (b) Lemma 2.11 using the asymptotes of the hyperbolic motion (The bounds on $\theta_{4}^{-}$and $\bar{\theta}_{4}^{+}$ require that $Q_{4}$ has a near collision with $Q_{3}$, which constrains $g_{4}$ ). So we only need to deal with the variables $\left(e_{4}, \ell_{3}\right)$ on a $\delta^{\prime}$-essential admissible surface. 
Finally, to prove that $(\mathcal{P} \mathcal{R} \widetilde{\mathbb{G}} \mathcal{P})^{-1}\left(S_{i+1}\right) \subset \widehat{S}_{i}$, we note that

$$
\begin{aligned}
(\mathcal{P} \mathcal{R} \widetilde{\mathbb{G}} \mathcal{P})^{-1}\left(S_{i+1}\right) & =\left\{\boldsymbol{x} \in \mathcal{P}^{-1}\left(\mathcal{S}_{i+1 / 2}\left(V_{0, i+1 / 2}\left(e_{4}^{*}\right)\right)\right): \pi_{e_{4}, \ell_{3}}(\mathcal{P} \mathcal{R} \widetilde{\mathbb{G}} \mathcal{P})(\boldsymbol{x}) \in \mathcal{C}_{2}\left(\delta^{\prime}\right)\right\} \\
& \subset \mathcal{P}^{-1}\left(\mathcal{S}_{i+1 / 2}\left(V_{0, i+1 / 2}\left(e_{4}^{*}\right)\right)\right) \subset \mathcal{P}^{-1}\left(\mathcal{S}_{i+1 / 2}\left(\mathcal{C}_{2}\left(\delta^{\prime}\right)\right)\right) \\
& =\mathcal{P}^{-1}\left(S_{i+1 / 2}\right)=\mathcal{S}_{i}\left(V_{2, i}\left(\tilde{e}_{4}\right)\right) \subset \mathcal{S}_{i}\left(\bar{V}_{2, i}\left(\tilde{e}_{4}\right)\right)=\widehat{S}_{i} .
\end{aligned}
$$

\section{The hyperbolicity of the Poincaré map}

In this section, we consider the hyperbolicity of the Poincaré map by studying the derivative of the local and global maps.

\subsection{The structure of the derivative of the global map and local map}

Lemma 3.1. Suppose the initial condition $\boldsymbol{x}_{\mu} \in U_{j}(\delta)$ satisfies $\mathbf{A L}$ and let $\widehat{\boldsymbol{x}}$ be the closest point to $\boldsymbol{x}_{\mu}$ in the set $U_{j}(\delta)$ with $\mu=0$, which leads to a collision between bodies 3 and 4. Then,

(a) there exist a constant $C$ independent of $\mu$, continuous vector-valued functions $\mathbf{l}_{j}(\widehat{\boldsymbol{x}})$ and $\mathbf{u}_{j}\left(\widehat{\boldsymbol{x}}, \bar{\theta}_{4}^{+}\right)$and a continuous matrix-valued function $B_{j}\left(\widehat{\boldsymbol{x}}, \bar{\theta}_{4}^{+}\right)$, where $\bar{\theta}_{4}^{+}$is the angle of the outgoing asymptote, such that $\left\|\mathbf{u}_{j}\right\|,\left\|\mathbf{1}_{j}\right\|,\left\|B_{j}\right\|<C$ and, as $1 / \chi \ll \mu \rightarrow 0$,

$$
d \mathbb{L}\left(\boldsymbol{x}_{\mu}\right)=\frac{1}{\mu}\left(\mathbf{u}_{j}\left(\widehat{\boldsymbol{x}}, \bar{\theta}_{4}^{+}\right)+o(1)\right) \otimes\left(\mathbf{l}_{j}(\widehat{\boldsymbol{x}})+o(1)\right)+B_{j}\left(\widehat{\boldsymbol{x}}, \bar{\theta}_{4}^{+}\right)+o(1) ;
$$

(b) moreover, there exist vectors $\hat{\mathbf{l}}_{j}$ and $\hat{u}_{j}$, and a matrix $\widehat{B}_{j}$ such that

$$
\mathbf{l}_{j}(\widehat{\boldsymbol{x}}) \rightarrow \hat{\mathbf{l}}_{j}, \quad \mathbf{u}_{j}\left(\widehat{\boldsymbol{x}}, \bar{\theta}_{4}^{+}\right) \rightarrow \hat{u}_{j}, \quad B_{j}\left(\widehat{\boldsymbol{x}}, \bar{\theta}_{4}^{+}\right) \rightarrow \widehat{B}_{j}, \quad \text { as } \delta, \tilde{\theta} \rightarrow 0,
$$

where $j=1,2$, meaning the first or the second collision.

The proof is given in $\$ 10.2$.

Lemma 3.2. Let $\boldsymbol{x}$ and $\boldsymbol{y}=\mathbb{G}(\boldsymbol{x})$ be the initial and final values of the global map $\mathbb{G}$ and suppose that they satisfy AG. Then,

(a) there exist continuous linear functionals $\overline{\mathbf{l}}_{j}(\boldsymbol{x})$ and $\overline{\overline{\mathbf{l}}}_{j}(\boldsymbol{x})$, continuous vectorfields $\overline{\mathbf{u}}_{j}(\boldsymbol{y})$ and $\overline{\mathbf{u}}_{j}(\boldsymbol{y})$, and non-vanishing constants $c_{1}$ and $c_{2}$ such that, as $1 / \chi \ll \mu \rightarrow 0$,

$$
d \mathbb{G}(\boldsymbol{x})=c_{1} \chi^{2}\left(\overline{\mathbf{u}}_{j}(\boldsymbol{y})+o(1)\right) \otimes\left(\overline{\mathbf{l}}_{j}(\boldsymbol{x})+o(1)\right)+c_{2} \chi\left(\overline{\overline{\mathbf{u}}}_{j}(\boldsymbol{y})+o(1)\right) \otimes\left(\overline{\overline{\mathbf{l}}}_{j}(\boldsymbol{x})+o(1)\right)+O(\mu \chi) ;
$$

(b) moreover, we have the following explicit expressions for the above vectors and functionals in Delaunay coordinates (see $\mathcal{V}$ in Notation 2.3):

$$
\overline{\overline{\mathbf{l}}}_{j}=\left(1,0_{1 \times 9}\right), \quad \overline{\mathbf{l}}_{j}=\left(-\frac{\widetilde{G}_{4, j} / \tilde{L}_{4, j}}{\tilde{L}_{4, j}^{2}+\widetilde{G}_{4, j}^{2}}, 0_{1 \times 7}, \frac{1}{\tilde{L}_{4, j}^{2}+\widetilde{G}_{4, j}^{2}}, \frac{1}{\tilde{L}_{4, j}}\right)
$$


and

$$
\overline{\overline{\mathbf{u}}}_{j}=\widetilde{w}:=\left(0,1,0_{1 \times 8}\right)^{T}, \quad \overline{\mathbf{u}}_{j}=w_{j}+c \widetilde{w}, \quad w_{j}=\left(0_{1 \times 8} ; 1, \frac{\hat{L}_{4, j}}{\hat{L}_{4, j}^{2}+\widehat{G}_{4, j}^{2}}\right)^{T}
$$

where $c$ is some constant, $\tilde{L}_{4, j}$ and $\widetilde{G}_{4, j}$ stand for the initial values of $L_{4, j}$ and $G_{4, j}$, and $\hat{L}_{4, j}$ and $\widehat{G}_{4, j}$ stand for the final values, and $\widetilde{w}$ and $w_{j}$ appeared in Definition 2.16, with $L_{4}=L_{3}$ when $\mu=0$ and $e_{4}=\sqrt{1+\left(G_{4} / L_{3}\right)^{2}}$.

Notation 3.3. We denote by $\hat{\overline{\mathbf{l}}}_{j}$ and $\hat{\overline{\mathbf{l}}}_{j}$ the vectors corresponding to $\overline{\overline{\mathbf{l}}}_{j}$ and $\overline{\mathbf{l}}_{j}$, respectively, when $L_{4, j}$ and $G_{4, j}$ are evaluated at Gerver's collision points.

The proof of Lemma 3.2 is in Appendix C.

\subsection{The non-degeneracy condition}

LEMma 3.4. The following non-degeneracy conditions are satisfied for $E_{3}^{*}=-\frac{1}{2}$, $e_{3}^{*}=\frac{1}{2}$ and $g_{3}^{*}=\frac{1}{2} \pi$ :

(a1) $\operatorname{span}\left(\hat{u}_{1}, \widehat{B}_{1}\left(\hat{\mathbf{l}}_{1}(\widetilde{w}) d \mathcal{R} w_{2}-\hat{\mathbf{l}}_{1}\left(d \mathcal{R} w_{2}\right) \widetilde{w}\right)\right)$ is transversal to $\operatorname{Ker}\left(\hat{\overline{\mathbf{l}}}_{1}\right) \cap \operatorname{Ker}\left(\hat{\overline{\mathbf{l}}}_{1}\right)$;

(a2) $d e_{4}\left(d \mathcal{R} w_{2}\right) \neq 0$;

(b1) $\operatorname{span}\left(\hat{u}_{2}, \widehat{B}_{2}\left(\hat{\mathbf{l}}_{2}(\widetilde{w}) w_{1}-\hat{\mathbf{l}}_{2}\left(w_{1}\right) \widetilde{w}\right)\right)$ is transversal to $\operatorname{Ker}\left(\hat{\overline{\mathbf{l}}}_{2}\right) \cap \operatorname{Ker}\left(\hat{\overline{\mathbf{l}}}_{2}\right)$;

(b2) $d e_{4}\left(w_{1}\right) \neq 0$;

(c) $\hat{\mathbf{l}}_{j} \cdot \widetilde{w} \neq 0, \hat{\mathbf{l}}_{2} \cdot w_{1} \neq 0, \hat{\mathbf{l}}_{1} \cdot d \mathcal{R} w_{2} \neq 0$ and $\hat{\overline{\mathbf{l}}}_{j} \cdot \widehat{\mathbf{u}}_{j} \neq 0, j=1,2$.

This lemma is proved in $\S 10.3$ and $\S 10.4$.

\subsection{Proof of Lemma 2.17, the expanding cones}

Consider for example the case where $\boldsymbol{x} \in U_{2}(\delta)$. We claim that, if $\delta$ and $\mu$ are small enough, then $d \mathbb{L}\left(\operatorname{span}\left(w_{1}, \widetilde{w}\right)\right)$ is transversal to $\operatorname{Ker} \overline{\mathbf{l}}_{2} \cap \operatorname{Ker} \overline{\overline{\mathbf{l}}}_{2}$. Indeed, take $\Gamma$ such that $\mathbf{l}_{2}(\Gamma)=0$. If $\Gamma=a w_{1}+\tilde{a} \widetilde{w}$, then $a \mathbf{l}_{2}\left(w_{1}\right)+\tilde{a} \mathbf{l}_{2}(\widetilde{w})=0$. It follows that the direction of $\Gamma$ is close to the direction of $\hat{\Gamma}=\hat{\mathbf{l}}_{2}(\widetilde{w}) w_{1}-\hat{\mathbf{l}}_{2}\left(w_{1}\right) \widetilde{w}$. Next, take $\widetilde{\Gamma}=b w_{1}+\tilde{b} \widetilde{w}$, where $b \mathbf{l}_{2}\left(w_{1}\right)+\tilde{b} \mathbf{l}_{2}(\widetilde{w}) \neq 0$. Then, the direction of $d \mathbb{L} \widetilde{\Gamma}$ is close to $\widehat{\mathbf{u}}_{2}$ and the direction of $d \mathbb{L}(\Gamma)$ is close to $B_{2}(\hat{\Gamma})$, so our claim follows from Lemma 3.4.

Thus, for any plane $\Pi$ close to $\operatorname{span}\left(w_{1}, \widetilde{w}\right)$, we have that $d \mathbb{L}(\Pi)$ is transversal to $\operatorname{Ker} \overline{\mathbf{l}}_{2} \cap \operatorname{Ker} \overline{\overline{\mathbf{l}}}_{2}$. Take any $Y \in \mathcal{K}_{2}$. Then either $Y$ and $w_{1}$ are linearly independent, or $Y$ and $\widetilde{w}$ are linearly independent. Hence, $d \mathbb{L}\left(\operatorname{span}\left(Y, w_{1}\right)\right)$ or $d \mathbb{L}(\operatorname{span}(Y, \widetilde{w}))$ is transversal to $\operatorname{Ker} \overline{\mathbf{l}}_{2} \cap \operatorname{Ker} \overline{\overline{\mathbf{l}}}_{2}$. Accordingly, either $\overline{\mathbf{l}}_{2}(d \mathbb{L}(Y)) \neq 0$ or $\overline{\overline{\mathbf{l}}}_{2}(d \mathbb{L}(Y)) \neq 0$. If $\overline{\mathbf{l}}_{2}(d \mathbb{L}(Y)) \neq 0$, then the direction of $d(\mathbb{G} \circ \mathbb{L})(Y)$ is close to $\overline{\mathbf{u}}$. If $\overline{\mathbf{l}}_{2}(d \mathbb{L}(Y))=0$ then the direction of 
$d(\mathbb{G} \circ \mathbb{L})(Y)$ is close to $\overline{\overline{\mathbf{u}}}$. Next, estimating $d \widetilde{\mathbb{G}}$, we have $d \widetilde{\mathbb{G}} \cdot \operatorname{span}(\overline{\mathbf{u}}, \overline{\overline{\mathbf{u}}})=\operatorname{span}(\overline{\mathbf{u}}, \overline{\overline{\mathbf{u}}})+o(1)$ (see Lemma 10.3). Next, by Definition 2.16, we get that $d \mathcal{R} \operatorname{span}(\overline{\mathbf{u}}, \overline{\overline{\mathbf{u}}}) \subset \mathcal{K}_{1}$.

So, in either of the two cases above, we have $d(\mathcal{R} \circ \widetilde{\mathbb{G}} \circ \mathbb{G} \circ \mathbb{L})(Y) \in \mathcal{K}_{1}$ and

$$
\|d(\mathcal{R} \circ \widetilde{\mathbb{G}} \circ \mathbb{G} \circ \mathbb{L})(Y)\| \geqslant c \chi\|Y\|
$$

This completes the proof in the case $\boldsymbol{x} \in U_{2}(\delta)$. The case of $\boldsymbol{x} \in U_{1}(\delta)$ is similar.

\section{Symplectic transformations and Poincaré sections}

In this section we define several Poincaré sections and perform symplectic transformations in the regions between the consecutive sections to make the Hamiltonian system suitable for doing calculations.

\subsection{The Poincaré coordinates}

We start with the Hamiltonian (1.1). The translation invariance enables us to remove one body in the Hamiltonian. We choose $Q_{2}$ as this body. We start with the symplectic form

$$
\begin{aligned}
\omega & =\sum_{i=1}^{4} d P_{i} \wedge d Q_{i} \\
& =d\left(P_{1}+P_{2}+P_{3}+P_{4}\right) \wedge d Q_{2}+d P_{1} \wedge d\left(Q_{1}-Q_{2}\right)+d P_{3} \wedge d\left(Q_{3}-Q_{2}\right)+d P_{4} \wedge d\left(Q_{4}-Q_{2}\right) \\
& =d\left(P_{1}+P_{2}+P_{3}+P_{4}\right) \wedge d Q_{2}+d P_{1} \wedge d q_{1}+d P_{3} \wedge d q_{3}+d P_{4} \wedge d q_{4}
\end{aligned}
$$

where we have used (2.1). If we choose the mass center of the four bodies as the origin, then $P_{1}+P_{2}+P_{3}+P_{4}=0$. Now the Hamiltonian becomes

$$
\begin{aligned}
H\left(q_{1}, P_{1} ; q_{3}, P_{3} ; q_{4}, P_{4}\right)= & P_{1}^{2}+\frac{1}{2}\left(1+\frac{1}{\mu}\right)\left(P_{3}^{2}+P_{4}^{2}\right)+\left(\left\langle P_{1}, P_{3}\right\rangle+\left\langle P_{1}, P_{4}\right\rangle+\left\langle P_{3}, P_{4}\right\rangle\right) \\
& -\frac{1}{\left|q_{1}\right|}-\frac{\mu}{\left|q_{3}\right|}-\frac{\mu}{\left|q_{4}\right|}-\frac{\mu}{\left|q_{1}-q_{3}\right|}-\frac{\mu}{\left|q_{1}-q_{4}\right|}-\frac{\mu^{2}}{\left|q_{3}-q_{4}\right|} .
\end{aligned}
$$

Restricted to the subspace where $P_{1}+P_{2}+P_{3}+P_{4}=0$, up to a factor $\mu$, the symplectic form is $\bar{\omega}$ defined in (2.4). We divide the whole Hamiltonian by $\mu$ to get

$$
\begin{aligned}
H\left(q_{1}, p_{1} ; q_{3}, p_{3} ; q_{4}, p_{4}\right)= & \mu p_{1}^{2}+\frac{\mu}{2}\left(1+\frac{1}{\mu}\right)\left(p_{3}^{2}+p_{4}^{2}\right)+\mu\left(\left\langle p_{1}, p_{3}\right\rangle+\left\langle p_{1}, p_{4}\right\rangle+\left\langle p_{3}, p_{4}\right\rangle\right) \\
& -\frac{1}{\mu\left|q_{1}\right|}-\frac{1}{\left|q_{3}\right|}-\frac{1}{\left|q_{4}\right|}-\frac{1}{\left|q_{1}-q_{3}\right|}-\frac{1}{\left|q_{1}-q_{4}\right|}-\frac{\mu}{\left|q_{3}-q_{4}\right|} .
\end{aligned}
$$


It can be checked that the Poincaré-Cartan 1-form is multiplied by a factor $\mu$ due to the coordinate change and the Hamiltonian canonical equation holds true in the new coordinates.

In the new coordinates the total angular momentum equals

$$
G=Q_{1} \times P_{1}-Q_{2} \times\left(P_{1}+P_{3}+P_{4}\right)+Q_{3} \times P_{3}+Q_{4} \times P_{4}=q_{1} \times P_{1}+q_{3} \times P_{3}+q_{4} \times P_{4} .
$$

Therefore, the conservation of angular momentum takes the form

$$
\sum_{j=3,1,4} q_{j} \times p_{j}=\text { const. }
$$

\subsection{More Poincaré sections}

When $Q_{4}$ is closer to $Q_{i}, i=1,2$, we treat its motion as a hyperbolic Kepler motion with focus at $Q_{i}$ and perturbed by $Q_{3}$ and $Q_{3-i}$.

Definition 4.1. We introduce one more set of coordinates

$$
\left\{\begin{array} { l } 
{ v _ { 3 } = p _ { 3 } + \frac { \mu } { 1 + \mu } ( p _ { 1 } + p _ { 4 } ) , } \\
{ v _ { 1 } = p _ { 1 } + p _ { 4 } , } \\
{ v _ { 4 } = \frac { 1 } { 1 + \mu } p _ { 4 } - \frac { \mu } { 1 + \mu } p _ { 1 } , }
\end{array} \quad \left\{\begin{array}{l}
x_{3}=q_{3}, \\
x_{1}=\frac{1}{1+\mu} q_{1}-\frac{\mu}{1+\mu} q_{3}+\frac{\mu}{1+\mu} q_{4}, \\
x_{4}=q_{4}-q_{1} .
\end{array}\right.\right.
$$

One can check that the transformation (4.2) is symplectic with respect to the symplectic form $\bar{\omega}$.

Notation 4.2. To distinguish the new set of coordinates from those of Definition 2.1, we use superscript $R$ (meaning right) and write

$$
\left(x_{3}, v_{3} ; x_{1}, v_{1} ; x_{4}, v_{4}\right)^{R}=\left(x_{3}^{R}, v_{3}^{R} ; x_{1}^{R}, v_{1}^{R} ; x_{4}^{R}, v_{4}^{R}\right)
$$

for the coordinates from Definition 2.1 and use superscript $L$ (meaning left) and write

$$
\left(x_{3}, v_{3} ; x_{1}, v_{1} ; x_{4}, v_{4}\right)^{L}=\left(x_{3}^{L}, v_{3}^{L} ; x_{1}^{L}, v_{1}^{L} ; x_{4}^{L}, v_{4}^{L}\right)
$$

for the coordinates from Definition 4.1. Notice that $\left(x_{3}, v_{3}\right)^{R}=\left(x_{3}, v_{3}\right)^{L}$, so we omit this superscript for simplicity.

Definition 4.3. (Further Poincaré sections.) We further define two more sections to cut the global map into three pieces (see Figure 3).

- Map (I) is the Poincaré map between the sections

$$
\left\{x_{4, \|}^{R}=-2, v_{4, \|}^{R}<0\right\} \quad \text { and } \quad\left\{x_{4, \|}^{R}=-\frac{1}{2} \chi, v_{4, \|}^{R}<0\right\} .
$$




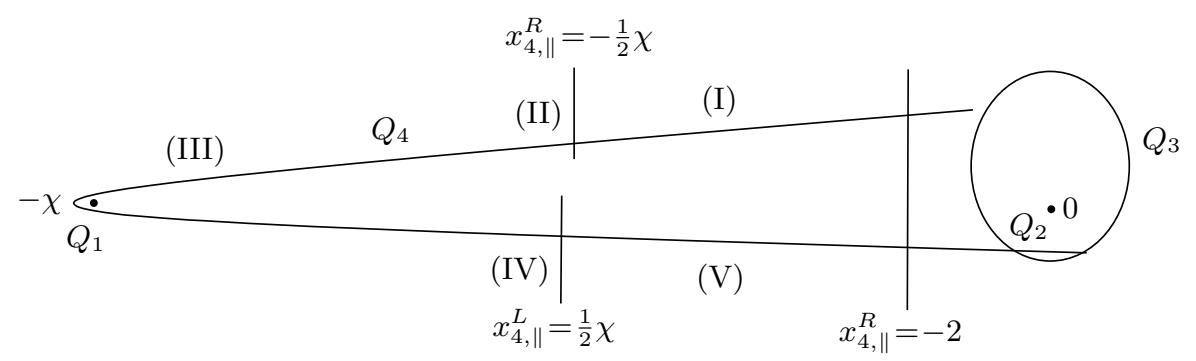

Figure 3. Poincaré sections.

- Map (III) is the Poincaré map between the sections

$$
\left\{x_{4, \|}^{R}=-\frac{1}{2} \chi, v_{4, \|}^{R}<0\right\} \quad \text { and } \quad\left\{x_{4, \|}^{L}=\frac{1}{2} \chi, v_{4, \|}^{L}>0\right\} .
$$

- Map (V) is the Poincaré map between the sections

$$
\left\{x_{4, \|}^{L}=\frac{1}{2} \chi, v_{4, \|}^{L}>0\right\} \quad \text { and } \quad\left\{x_{4, \|}^{R}=-2, v_{4, \|}^{R}>0\right\} .
$$

- We also introduce map (II) to change coordinates from right to the left on the section $\left\{x_{4, \|}^{R}=-\frac{1}{2} \chi, v_{4, \|}^{R}<0\right\}$ and map (IV) to change coordinates from left to the right on the section $\left\{x_{4, \|}^{L}=\frac{1}{2} \chi, v_{4, \|}^{L}>0\right\}$.

Remark 4.4. (1) The two sections $\left\{x_{4, \|}^{R}=-\frac{1}{2} \chi, v_{4, \|}^{R}<0\right\}$ and $\left\{x_{4, \|}^{L}=\frac{1}{2} \chi, v_{4, \|}^{L}>0\right\}$ lie almost at the midpoint of $Q_{1}$ and $Q_{2}$.

(2) In $\S 4.3$ and $\S 4.4$, we will treat the equations of motion as three Kepler motions $\left(x_{i}, v_{i}\right)^{R, L}, i=3,1,4$, with perturbations. When perturbation is neglected, the orbit of $x_{4}^{R}$ is a hyperbola focused at the origin and opening to the left, while the orbit of $x_{4}^{L}$ is a hyperbola focused at origin and opening to the right.

In the following subsections we describe the suitable changes of variables adapted to maps (I), (III) and (V), as well as the local map $\mathbb{L}$.

\subsection{Hamiltonian of the right case, when $Q_{4}$ is closer to $Q_{2}$}

We write the Hamiltonian in terms of three Kepler motions with perturbations.

Lemma 4.5. In the coordinates (2.3), the Hamiltonian for maps (I) and (V) has the form

$$
\begin{aligned}
& H\left(x_{3}, v_{3} ; x_{1}, v_{1} ; x_{4}, v_{4}\right) \\
& \quad=\left(\frac{\mu(1+\mu)}{1+2 \mu} v_{1}^{2}-\frac{2 \mu+1}{\mu\left|x_{1}\right|}\right)+\left(\frac{1+\mu}{2} v_{3}^{2}-\frac{1}{\left|x_{3}\right|}\right)+\left(\frac{1+2 \mu}{2(1+\mu)} v_{4}^{2}-\frac{1+\mu}{\left|x_{4}\right|}\right)+U^{R},
\end{aligned}
$$


where

$$
U^{R}\left(x_{3}, x_{1}, x_{4}\right)=\frac{2 \mu+1}{\mu\left|x_{1}\right|}-\left(\frac{1}{a}+\frac{1}{b}+\frac{1}{c}\right)+\frac{1+\mu}{\left|x_{4}\right|}-\left(\frac{1}{d}+\frac{\mu}{e}\right) .
$$

with

$$
\begin{gathered}
a=\mu\left|x_{1}+\frac{\mu}{1+2 \mu} x_{4}+\frac{\mu}{1+\mu} x_{3}\right|, \quad b=\left|x_{1}+\frac{\mu}{1+2 \mu} x_{4}-\frac{1}{1+\mu} x_{3}\right|, \\
c=\left|x_{1}-\frac{1+\mu}{1+2 \mu} x_{4}\right|, \quad d=\left|x_{4}+\frac{\mu x_{3}}{1+\mu}\right|, \quad e=\left|\frac{x_{3}}{1+\mu}-x_{4}\right|
\end{gathered}
$$

In Delaunay variables, we have

$$
H\left(L_{3}, \ell_{3}, G_{3}, g_{3} ; x_{1}, v_{1} ; L_{4}, \ell_{4}, G_{4}, g_{4}\right)=\left(\frac{v_{1}^{2}}{2 m_{1 R}}-\frac{k_{1 R}}{\left|x_{1}\right|}\right)-\frac{m_{3 R} k_{3 R}^{2}}{2 L_{3}^{2}}+\frac{m_{4 R} k_{4 R}^{2}}{2 L_{4}^{2}}+U^{R},
$$

where

$$
\begin{gathered}
m_{1 R}=\frac{1+2 \mu}{2 \mu(1+\mu)}, \quad m_{3 R}=\frac{1}{1+\mu}, \quad m_{4 R}=\frac{1+\mu}{1+2 \mu}, \\
k_{1 R}=\frac{1+2 \mu}{\mu}, \quad k_{3 R}=1, \quad k_{4 R}=1+\mu .
\end{gathered}
$$

\subsection{Hamiltonian of the left case, when $Q_{4}$ is closer to $Q_{1}$}

In this section, we explain the choice of the Jacobi coordinates (4.2) and derive the corresponding Hamiltonian. When $Q_{4}$ is moving between the sections $\left\{x_{4, \|}^{R}=-\frac{1}{2} \chi\right\}$ and $\left\{x_{4, \|}^{L}=\frac{1}{2} \chi\right\}$ and turns around $Q_{1}$, we treat $Q_{4}$ 's motion as an approximate hyperbola with focus at $Q_{1}$.

LEMMA 4.6. In the coordinates (4.2), the Hamiltonian for map (III) becomes

$$
\begin{aligned}
& H\left(x_{3}, v_{4} ; x_{1}, v_{1} ; x_{4}, v_{4}\right) \\
& \quad=\frac{\mu}{1+\mu} v_{1}^{2}-\frac{(1+\mu)^{2}}{\mu\left|x_{1}\right|}+\frac{\mu}{2}\left(1+\frac{1}{\mu}\right)\left(v_{3}^{2}+v_{4}^{2}\right)-\frac{1}{\left|x_{3}\right|}-\frac{1}{\left|x_{4}\right|}+U^{L},
\end{aligned}
$$

where

$$
U^{L}\left(x_{3}, x_{1}, x_{4}\right)=\frac{(1+\mu)^{2}}{\mu\left|x_{1}\right|}-\left(\frac{1}{a}+\frac{1}{b}+\frac{1}{c}+\frac{\mu}{d}\right),
$$

with

$$
\begin{gathered}
a=\mu\left|x_{1}+\frac{\mu}{1+\mu} x_{3}-\frac{\mu}{1+\mu} x_{4}\right|, \quad b=\left|x_{1}+\frac{\mu}{1+\mu} x_{3}+\frac{1}{1+\mu} x_{4}\right|, \\
c=\left|x_{1}-\frac{1}{1+\mu} x_{3}-\frac{\mu}{1+\mu} x_{4}\right|, \quad d=\left|x_{1}-\frac{1}{1+\mu} x_{3}+\frac{1}{1+\mu} x_{4}\right| .
\end{gathered}
$$

In Delaunay coordinates, we have

$$
H\left(L_{3}, \ell_{3}, G_{3}, g_{3} ; x_{1}, v_{1} ; L_{4}, \ell_{4}, G_{4}, g_{4}\right)=\left(\frac{v_{1}^{2}}{2 m_{1 L}}-\frac{k_{1 L}}{\left|x_{1}\right|}\right)-\frac{m_{3 L} k_{3 L}^{2}}{2 L_{3}^{2}}+\frac{m_{4 L} k_{4 L}^{2}}{2 L_{4}^{2}}+U^{L},
$$

where

$$
m_{1 L}=\frac{1+\mu}{2 \mu}, \quad m_{3 L}=m_{4 L}=\frac{1}{1+\mu}, \quad k_{1 L}=\frac{(1+\mu)^{2}}{\mu}, \quad k_{3 L}=k_{4 L}=1 .
$$




\subsection{Hamiltonian of the local map, away from close encounter}

We cut the local map into three pieces by introducing a new section $\left|q_{3}-q_{4}\right|=\mu^{\kappa}$, with $\frac{1}{3}<\kappa<\frac{1}{2}$. The restriction $\kappa<\frac{1}{2}$ comes from the proof of Lemma 10.3, where we need $\mu^{1-2 \kappa}$ to be small, and the restriction $\kappa>\frac{1}{3}$ comes from the proof of Lemma 10.2, where we need $\mu^{3 \varkappa-1}$ to be small.

When $Q_{3}$ and $Q_{4}$ are moving outside the circle $\left|q_{3}-q_{4}\right|=\mu^{\kappa}$, we use the same transformation as (2.3), but different ways of grouping terms. So, we get the following from the first equality of (4.3).

Lemma 4.7. In the coordinates (2.3), assume that $\left|x_{3}\right|,\left|x_{4}\right|<2$ and $\left|x_{1}\right| \sim \chi$. Then, the Hamiltonian can be written in the following form:

$$
\begin{aligned}
& H\left(x_{1}, v_{1} ; x_{3}, v_{3} ; x_{4}, v_{4}\right)^{R} \\
& =\left(\frac{\mu(1+\mu)}{1+2 \mu} v_{1}^{2}-\frac{2 \mu+1}{\mu\left|x_{1}\right|}\right)+\left(\frac{1+\mu}{2} v_{3}^{2}-\frac{1}{\left|x_{3}\right|}\right)+\left(\frac{1+2 \mu}{2(1+\mu)} v_{4}^{2}-\frac{1+\mu}{\left|x_{4}\right|}\right) \\
& \quad-\frac{\mu}{\left|x_{3} /(1+\mu)-x_{4}\right|}+V_{\text {out }}\left(x_{3}, x_{1}, x_{4}\right),
\end{aligned}
$$

where

$$
V_{\text {out }}\left(x_{3}, x_{1}, x_{4}\right)=\left(\frac{1+\mu}{\left|x_{4}\right|}-\frac{1}{a}\right)+\left(\frac{2 \mu+1}{\mu\left|x_{1}\right|}-\frac{1}{b}-\frac{1}{c}-\frac{1}{d}\right)
$$

with

$$
\begin{gathered}
a=\left|x_{4}+\frac{\mu x_{3}}{1+\mu}\right|, \quad b=\mu\left|x_{1}+\frac{\mu}{1+2 \mu} x_{4}+\frac{\mu}{1+\mu} x_{3}\right|, \\
c=\left|x_{1}+\frac{\mu}{1+2 \mu} x_{4}-\frac{1}{1+\mu} x_{3}\right|, \quad d=\left|x_{1}-\frac{1+\mu}{1+2 \mu} x_{4}\right| .
\end{gathered}
$$

In Delaunay coordinates, we have

$$
\begin{aligned}
& H\left(x_{1}, v_{1} ; L_{3}, \ell_{3}, G_{3}, g_{3} ; L_{4}, \ell_{4}, G_{4}, g_{4}\right)^{R} \\
& \quad=\left(\frac{v_{1}^{2}}{2 m_{1 R}}-\frac{k_{1 R}}{\left|x_{1}\right|}\right)-\frac{m_{3 R} k_{3 R}^{2}}{2 L_{3}^{2}}+\frac{m_{4 R} k_{4 R}^{2}}{2 L_{4}^{2}}-\frac{\mu}{\left|x_{3} /(1+\mu)-x_{4}\right|}+V_{\text {out }} .
\end{aligned}
$$

\subsection{Hamiltonian of the local map, close encounter}

When $Q_{3}$ and $Q_{4}$ are moving inside the circle $\left|q_{3}-q_{4}\right|=\mu^{\kappa}$, we derive the Hamiltonian system describing the relative motion of $Q_{3}$ and $Q_{4}$. We start with the Hamiltonian (4.1), and make the following symplectic changes to convert to the coordinates of relative motion and motion of the center of mass

$$
\left\{\begin{array} { l } 
{ q _ { - } = \frac { 1 } { 2 } ( q _ { 3 } - q _ { 4 } ) , } \\
{ q _ { + } = \frac { 1 } { 2 } ( q _ { 3 } + q _ { 4 } ) , } \\
{ q _ { 1 } = q _ { 1 } }
\end{array} \quad \text { and } \quad \left\{\begin{array}{l}
p_{-}=p_{3}-p_{4}, \\
p_{+}=p_{3}+p_{4}, \\
p_{1}=p_{1}
\end{array}\right.\right.
$$


The symplectic form is now

$$
\bar{\omega}=d p_{1} \wedge d q_{1}+d p_{+} \wedge d \mathbf{q}+d p_{-} \wedge d q_{-} .
$$

Lemma 4.8. In the coordinates (4.10), we have $\left|q_{-}\right| \leqslant 2 \mu^{\kappa}$ inside the circle $\left|q_{-}\right|=2 \mu^{\kappa}$ and the following expression of the Hamiltonian (4.1):

$$
H\left(q_{1}, p_{1} ; q_{-}, p_{-} ; q_{+}, p_{+}\right)=\left(\mu p_{1}^{2}-\frac{1+2 \mu}{\mu\left|q_{1}\right|}\right)+\left(\frac{1+2 \mu}{4} p_{+}^{2}-\frac{2}{\left|q_{+}\right|}\right)+\left(\frac{1}{4} p_{-}^{2}-\frac{\mu}{2\left|q_{-}\right|}\right)+V_{\mathrm{in}},
$$

where

$$
\begin{aligned}
V_{\text {in }} & =\mu\left\langle p_{1}, p_{+}\right\rangle-\frac{1}{\left|q_{+}-q_{-}\right|}-\frac{1}{\left|q_{+}+q_{-}\right|}-\frac{1}{\left|q_{1}-q_{+}+q_{-}\right|}-\frac{1}{\left|q_{1}-q_{+}-q_{-}\right|}+\frac{2}{\left|q_{1}\right|}+\frac{2}{\left|q_{+}\right|} \\
& =\mu\left\langle p_{1}, p_{+}\right\rangle-\frac{3\left\langle q_{+}, q_{-}\right\rangle^{2}}{2\left|q_{+}\right|^{5}}+\frac{\left|q_{-}\right|^{2}}{\left|q_{+}\right|^{3}}-\frac{\left\langle q_{1}, q_{+}\right\rangle}{\left|q_{1}\right|^{3}}+O\left(\left|q_{-}\right|^{3}+\frac{1}{\left|q_{1}\right|^{3}}\right) .
\end{aligned}
$$

The $O$ expression holds as $\left|q_{-}\right| \rightarrow 0$ and $\left|q_{1}\right| \rightarrow \infty$.

We can convert the term $\frac{1}{4} p_{-}^{2}-\mu / 2\left|q_{-}\right|$into $\mu^{2} / 4 L_{-}^{2}$, in terms of Delaunay coordinates.

\section{Statement of the main technical proposition}

In this section, we give the statement of our calculation of matrices needed in the proof of the global map. We use the coordinate system $\left(L_{3}, \ell_{3}, G_{3}, g_{3} ; x_{1}, v_{1} ; G_{4}, g_{4}\right)$ to do the calculation. In the following, the superscript "i" means "initial" and "f" means "final".

Notation 5.1. To avoid many $O$ notations in our estimates, we introduce the following conventions.

- We use the notation $a \lesssim b$ if $a=O(b)$ or equivalently $|a| \leqslant C|b|$ for some constant $C$ independent of $\chi$ and $\mu$, and the notation $a \sim b$ if both $a \lesssim b$ and $b \lesssim a$ hold.

- We also generalize this notation to vectors and matrices. For two vectors $A, B \in \mathbb{R}^{n}$, we write $A \lesssim B$ if $A_{i} \lesssim B_{i}$ holds for each entry $A_{i}$ and $B_{i}$ of $A$ and $B$, respectively, and write $A \sim B$ if $A \lesssim B$ and $B \lesssim A$ hold. Similarly for matrices.

- For a matrix [H], we refer to its blocks as

$$
\left[\begin{array}{l|l|l}
\sharp_{33} & \sharp_{31} & \sharp_{34} \\
\hline \sharp_{13} & \sharp_{11} & \sharp_{14} \\
\hline \sharp_{43} & \sharp_{41} & \sharp_{44}
\end{array}\right],
$$

and its $(i, j)$-th entry as $[\sharp](i, j), i, j=1,2, \ldots, 10$. 
- Moreover, when we use "¿", there may be some entries in the vector or matrix, for which we have an estimate in the sense of $\sim$. Those entries will be important to show that the $\chi^{2}$ and $\chi$ terms in Lemma 3.2 do not vanish. For those entries, we use bold font.

Proposition 5.2. Under the assumption AG, we have the following:

(a.1) The derivative of the global map is the product of five $10 \times 10$ matrices

$$
d \mathbb{G}=(\mathrm{V})(\mathrm{IV})(\mathrm{III})(\mathrm{II})(\mathrm{I})
$$

having the following form

$$
\begin{aligned}
(\mathrm{I}) & =\left(\operatorname{Id}_{10}+\chi u_{1}^{\mathrm{f}} \otimes l_{1}^{\mathrm{f}}\right) N_{1}\left(\operatorname{Id}_{10}-u_{1}^{\mathrm{i}} \otimes l_{1}^{\mathrm{i}}\right) \lesssim\left(\operatorname{Id}_{10}+\chi u \otimes l\right) N_{1}\left(\operatorname{Id}_{10}+u_{1}^{\mathrm{i}} \otimes l_{1}^{\mathrm{i}}\right), \\
(\mathrm{II}) & =\left(\chi u_{i i i} \otimes l_{i i i}+A\right) L \cdot R^{-1}\left(\chi u_{i} \otimes l_{i}+C\right), \\
(\mathrm{III}) & =\left(\operatorname{Id}_{10}+\chi u_{3}^{\mathrm{f}} \otimes l_{3}^{\mathrm{f}}\right) N_{3}\left(\operatorname{Id}_{10}-\chi u_{3}^{\mathrm{i}} \otimes l_{3}^{\mathrm{i}}\right) \lesssim\left(\operatorname{Id}_{10}+\chi u \otimes l\right) N_{3}\left(\operatorname{Id}_{10}+\chi u \otimes l^{\prime}\right), \\
(\mathrm{IV}) & =\left(\chi u_{i i i^{\prime}} \otimes l_{i i i^{\prime}}+A\right) R \cdot L^{-1}\left(\chi u_{i^{\prime}} \otimes l_{i^{\prime}}+C\right), \\
(\mathrm{V}) & =\left(\operatorname{Id}_{10}+u_{5}^{\mathrm{f}} \otimes l_{5}^{\mathrm{f}}\right) N_{5}\left(\operatorname{Id}_{10}-\chi u_{5}^{\mathrm{i}} \otimes l_{5}^{\mathrm{i}}\right) \lesssim\left(\operatorname{Id}_{10}+u_{1}^{\mathrm{i}} \otimes l_{1}^{\mathrm{i}}\right) N_{5}\left(\operatorname{Id}_{10}+\chi u \otimes l^{\prime}\right),
\end{aligned}
$$

(a.2) where

$$
\begin{aligned}
u_{1}^{\mathrm{f}}, u_{3}^{\mathrm{f}}, u_{3}^{\mathrm{i}}, u_{5}^{\mathrm{i}} \lesssim u & :=\left(\frac{1}{\chi^{3}},-\mathbf{1}, \frac{1}{\chi^{3}}, \frac{1}{\chi^{3}} ; \mu, \frac{\mu}{\chi}, \frac{1}{\mu \chi^{2}}, \frac{1}{\chi^{3}} ; \frac{1}{\chi^{2}}, \frac{1}{\chi^{2}}\right)_{10 \times 1}^{T}, \\
l_{1}^{\mathrm{f}}, l_{3}^{\mathrm{f}} \lesssim l & :=\left(\mathbf{1}, \frac{1}{\chi^{3}}, \frac{1}{\chi^{3}}, \frac{1}{\chi^{3}} ; \frac{1}{\mu \chi^{2}}, \frac{1}{\chi^{3}}, \mu, \frac{\mu}{\chi} ; \frac{1}{\chi}, \frac{1}{\chi}\right)_{1 \times 10}, \\
-l_{3}^{\mathrm{i}},-l_{5}^{\mathrm{i}} \lesssim l^{\prime}: & =\left(\mathbf{1}, \frac{1}{\chi^{3}}, \frac{1}{\chi^{3}}, \frac{1}{\chi^{3}} ; \frac{1}{\chi}, \frac{1}{\chi^{3}}, \mu, \frac{\mu}{\chi} ; \frac{1}{\chi}, \frac{1}{\chi}\right)_{1 \times 10}, \\
l_{i i i}, l_{i i i^{\prime}} & \lesssim\left(0_{1 \times 8} ; \frac{1}{\chi^{2}}, \frac{\mathbf{1}}{\chi}, \frac{1}{\chi}, \mathbf{1}\right)_{1 \times 12}, \\
u_{i} & =\left(01 \times 9, \frac{L_{4}}{2 m_{4 R}^{2} k_{4 R}^{2}}, 0, \frac{\mathbf{1}}{\chi}\right)_{12 \times 1}^{T}, \\
u_{i^{\prime}} & =\left(01 \times 9, \frac{L_{4}}{2 m_{4 L}^{2} k_{4 L}^{2}}, 0, \frac{\mathbf{1}}{\chi}\right)_{12 \times 1}^{T}, \\
l_{i} & \lesssim\left(\mathbf{1}, \frac{1}{\chi^{3}}, \frac{1}{\chi^{3}}, \frac{1}{\chi^{3}} ; \frac{1}{\mu \chi^{2}}, \frac{1}{\chi^{3}}, \mu, \frac{\mu}{\chi} ; \mathbf{1}, \mathbf{1}\right)_{1 \times 10}, \\
l_{i^{\prime}} & \lesssim\left(\frac{1}{\chi}, \frac{1}{\chi^{4}}, \frac{1}{\chi^{4}}, \frac{1}{\chi^{4}} ; \frac{1}{\mu \chi^{3}}, \frac{1}{\chi^{4}}, \frac{\mu}{\chi}, \frac{\mu^{2}}{\chi^{2}} ; \mathbf{1}, \mathbf{1}\right)_{1 \times 10} \\
u_{5}^{\mathrm{f}}, u_{1}^{\mathrm{i}} & \lesssim\left(\mu, 1, \mu, \mu ; \mu, \frac{\mu}{\chi}, \frac{1}{\mu \chi^{2}}, \frac{1}{\chi^{3}} ; \mu, \mu\right)_{10 \times 1}^{T}
\end{aligned}
$$




$$
l_{5}^{\mathrm{f}}, l_{1}^{\mathrm{i}} \lesssim\left(1, \mu, \mu, \mu ; \frac{1}{\mu \chi^{2}}, \frac{1}{\chi^{3}}, \mu, \frac{\mu}{\chi} ; 1,1\right)_{1 \times 10}
$$

(a.3) and

$$
\begin{aligned}
& A=\left[\begin{array}{c|cccc}
\operatorname{Id}_{8 \times 8} & 0_{8 \times 1} & 0_{8 \times 1} & 0_{8 \times 1} & 0_{8 \times 1} \\
\hline 0_{1 \times 8} & 0 & 0 & 0 & 0 \\
0_{1 \times 8} & O\left(1 / \chi^{2}\right) & O\left(1 / \chi^{2}\right) & O(1) & O(1)
\end{array}\right]_{10 \times 12}, \\
& C=\left[\begin{array}{c|c}
\operatorname{Id}_{8 \times 8} & 0_{8 \times 2} \\
\hline 0_{1 \times 8} & 0_{1 \times 2} \\
0_{1 \times 8} & O(1)_{1 \times 2} \\
\breve{l}_{i} & O(1 / \chi)_{1 \times 2} \\
0_{1 \times 8} & 0_{1 \times 2}
\end{array}\right]_{12 \times 10}
\end{aligned}
$$

with

$$
\breve{l}_{i} \lesssim\left(1, \frac{1}{\chi^{3}}, \frac{1}{\chi^{3}}, \frac{1}{\chi^{3}} ; \frac{1}{\mu \chi^{2}}, \frac{1}{\chi^{3}}, \mu, \frac{\mu}{\chi}\right)_{1 \times 8} ;
$$

(a.4) the matrices $R$ and $L$ are the derivative matrices of the transformations (2.3) and (4.2), respectively, and, in the coordinates $\left(x_{3}, v_{3} ; x_{1}, v_{1} ; x_{4}, v_{4}\right)$, they have the following expression:

$$
R \cdot L^{-1}, L \cdot R^{-1}=\left[\begin{array}{ccccc}
\mathrm{Id}_{4} & 0 & 0 & 0 & 0 \\
0 & m_{ \pm} \mathrm{Id}_{2} & 0 & \mp \frac{2 \mu}{1+2 \mu} \mathrm{Id}_{2} & 0 \\
0 & 0 & m_{\mp} \mathrm{Id}_{2} & 0 & \mp \operatorname{Id}_{2} \\
0 & \pm \mathrm{Id}_{2} & 0 & m_{\mp} \mathrm{Id}_{2} & 0 \\
0 & 0 & \pm \frac{2 \mu}{1+2 \mu} \mathrm{Id}_{2} & 0 & m_{ \pm} \mathrm{Id}_{2}
\end{array}\right]_{12 \times 12}
$$

where

$$
m_{+}=\frac{1+\mu}{1+2 \mu} \quad \text { and } \quad m_{-}=\frac{1}{1+\mu}
$$

and we choose the upper sign for $R \cdot L^{-1}$ and the lower sign for $L \cdot R^{-1}$ when we need to make a choice in \pm or $\mp$; 
(a.5) the following estimates hold:

$$
\begin{aligned}
& N_{1}-\operatorname{Id}_{10} \lesssim\left[\begin{array}{cccc|cccc|cc}
\mu & \mu & \mu & \mu & \mu / \chi & \mu / \chi & \mu^{2} & \mu / \chi & \mu & \mu \\
\mu \chi & \mu^{2} \chi & \mu^{2} \chi & \mu^{2} \chi & \mu^{2} & \mu^{2} & \mu \chi & \mu & \mu^{2} \chi & \mu^{2} \chi \\
\mu & \mu & \mu & \mu & \mu / \chi & \mu / \chi & \mu^{2} & \mu / \chi & \mu & \mu \\
\mu & \mu & \mu & \mu & \mu / \chi & \mu / \chi & \mu^{2} & \mu / \chi & \mu & \mu \\
\hline \mu \chi & \mu^{2} \chi & \mu^{2} \chi & \mu^{2} \chi & \mu^{2} & \mu^{2} & \mu \chi & \mu^{2} & \mu^{2} \chi & \mu^{2} \chi \\
\mu & \mu^{2} & \mu^{2} & \mu^{2} & \mu / \chi & 1 / \chi & \mu^{2} & \mu \chi & \mu & \mu \\
1 / \mu \chi & 1 / \chi & 1 / \chi & 1 / \chi & 1 / \mu \chi^{2} & 1 / \chi^{2} & 1 / \chi & \mu / \chi & 1 / \chi & 1 / \chi \\
1 / \chi & \mu / \chi & \mu / \chi & \mu / \chi & 1 / \chi^{2} & 1 / \mu \chi^{2} & \mu / \chi & 1 / \chi & 1 / \chi & 1 / \chi \\
\hline 1 & \mu & \mu & \mu & 1 / \chi & 1 / \chi & \mu & \mu & \mathbf{1} & \mathbf{1} \\
1 & \mu & \mu & \mu & 1 / \chi & 1 / \chi & \mu & \mu & \mathbf{1} & \mathbf{1}
\end{array}\right]_{10 \times 10}, \\
& N_{3}-\operatorname{Id}_{10} \lesssim\left[\begin{array}{cccc|cccc|cc}
\mu / \chi & 1 / \chi^{2} & 1 / \chi^{2} & 1 / \chi^{2} & 1 / \mu \chi^{3} & 1 / \chi^{3} & \mu / \chi & \mu / \chi^{2} & 1 / \chi^{2} & 1 / \chi^{2} \\
\mu \chi & \mu / \chi & \mu / \chi & \mu / \chi & 1 / \mu \chi & 1 / \chi & \mu \chi & \mu & 1 & 1 \\
\mu / \chi & 1 / \chi^{2} & 1 / \chi^{2} & 1 / \chi^{2} & 1 / \mu \chi^{3} & 1 / \chi^{3} & \mu / \chi & \mu / \chi^{2} & 1 / \chi^{2} & 1 / \chi^{2} \\
\mu / \chi & 1 / \chi^{2} & 1 / \chi^{2} & 1 / \chi^{2} & 1 / \mu \chi^{3} & 1 / \chi^{3} & \mu / \chi & \mu / \chi^{2} & 1 / \chi^{2} & 1 / \chi^{2} \\
\hline \mu \chi & \mu / \chi & \mu / \chi & \mu / \chi & 1 / \chi & \mu / \chi & \mu \chi & \mu^{2} & \mu & \mu \\
\mu & \mu / \chi^{2} & \mu / \chi^{2} & \mu / \chi^{2} & \mu / \chi & 1 / \chi & \mu^{2} & \mu \chi & \mu & \mu \\
1 / \mu \chi & 1 / \mu \chi^{3} & 1 / \mu \chi^{3} & 1 / \mu \chi^{3} & 1 / \mu \chi^{2} & 1 / \chi^{2} & 1 / \chi & \mu / \chi & 1 / \chi & 1 / \chi \\
1 / \chi & 1 / \chi^{3} & 1 / \chi^{3} & 1 / \chi^{3} & 1 / \chi^{2} & 1 / \mu \chi^{2} & \mu / \chi & 1 / \chi & 1 / \chi & 1 / \chi \\
\hline 1 & 1 / \chi^{2} & 1 / \chi^{2} & 1 / \chi^{2} & 1 / \chi & 1 / \chi & \mu & \mu & \mathbf{1} & \mathbf{1} \\
1 & 1 / \chi^{2} & 1 / \chi^{2} & 1 / \chi^{2} & 1 / \chi & 1 / \chi & \mu & \mu & \mathbf{1} & \mathbf{1}
\end{array}\right]_{10 \times 10} \\
& N_{5}-\operatorname{Id}_{10} \lesssim\left[\begin{array}{cccc|cccc|cc}
\mu^{2} \chi & \mu & \mu & \mu & 1 / \chi & \mu / \chi & \mu^{2} \chi & \mu^{2} & \mu & \mu \\
\mu \chi & \mu & \mu & \mu & 1 / \mu \chi & 1 / \chi & \mu \chi & \mu & 1 & 1 \\
\mu^{2} \chi & \mu & \mu & \mu & 1 / \chi & \mu / \chi & \mu^{2} \chi & \mu^{2} & \mu & \mu \\
\mu^{2} \chi & \mu & \mu & \mu & 1 / \chi & \mu / \chi & \mu^{2} \chi & \mu^{2} & \mu & \mu \\
\hline \mu \chi & \mu^{2} & \mu^{2} & \mu^{2} & 1 / \chi & \mu / \chi & \mu \chi & \mu^{2} & \mu & \mu \\
\mu & \mu^{2} / \chi & \mu^{2} / \chi & \mu^{2} / \chi & \mu / \chi & 1 / \chi & \mu^{2} & \mu \chi & \mu & \mu \\
\mu^{2} & \mu / \chi & \mu / \chi & \mu / \chi & 1 / \mu \chi^{2} & 1 / \chi^{2} & \mu^{2} & \mu / \chi & 1 / \chi & 1 / \chi \\
\mu^{2} & \mu / \chi & \mu / \chi & \mu / \chi & 1 / \chi^{2} & 1 / \mu \chi^{2} & \mu^{2} & 1 / \chi & 1 / \chi & 1 / \chi \\
\hline \mu^{2} \chi & \mu & \mu & \mu & 1 / \chi & 1 / \chi & \mu^{2} \chi & \mu & \mathbf{1} & \mathbf{1} \\
\mu^{2} \chi & \mu & \mu & \mu & 1 / \chi & 1 / \chi & \mu^{2} \chi & \mu & \mathbf{1} & \mathbf{1}
\end{array}\right]_{10 \times 10}
\end{aligned}
$$


(b) Moreover, for the $\mathbf{1}$ entries in (a.2), we have the following exact estimates:

(b.1) As $1 / \chi \ll \mu \rightarrow 0$, we have

$$
\begin{aligned}
& u_{1,3}^{f}, u_{3,5}^{\mathrm{i}}, u \rightarrow\left(0,1,0_{1 \times 8}\right)_{10 \times 1}^{T}=\widetilde{w}, \\
& u_{i i i} \rightarrow\left(0_{1 \times 8} ; 1, \frac{1}{\tilde{L}_{4, j}}\right)_{10 \times 1}^{T}, \\
& u_{i i i^{\prime}} \rightarrow\left(0_{1 \times 8} ; 1, \frac{\hat{L}_{4, j}}{\widehat{G}_{4, j}^{2}+\hat{L}_{4, j}^{2}}\right)_{10 \times 1}^{T}=w_{j}, \\
& l_{1,3}^{\mathrm{f}}, l_{3,5}^{\mathrm{i}}, l, l^{\prime} \rightarrow\left(1,0_{1 \times 9}\right)_{1 \times 10}=\hat{\overline{\overline{\mathbf{l}}}}_{j}, \\
& l_{i} \rightarrow\left(\frac{\widetilde{G}_{4, j} / \tilde{L}_{4, j}}{\tilde{L}_{4, j}^{2}+\widetilde{G}_{4, j}^{2}}, 0_{1 \times 7},-\frac{1}{\tilde{L}_{4, j}^{2}+\widetilde{G}_{4, j}^{2}},-\frac{1}{\tilde{L}_{4, j}}\right)_{1 \times 10}=\hat{\overline{\mathbf{l}}}_{j} .
\end{aligned}
$$

Here, $j=1,2$ means the first and second collisions in Gerver's construction. $\tilde{L}_{4}$ and $\widetilde{G}_{4}$ are the values of the Delaunay coordinates at the initial point for the global map and $\hat{L}_{4}$ and $\widehat{G}_{4}$ are the values of the Delaunay coordinates at the final point.

(b.2) In addition, as $1 / \chi \ll \mu \rightarrow 0$, we have

$$
\begin{aligned}
l_{i^{\prime}} & \rightarrow\left(0_{1 \times 8}, \frac{1}{\tilde{L}_{4, j}^{2}},-\frac{1}{\tilde{L}_{4, j}}\right)_{1 \times 10}, \\
u_{i^{\prime}} & =\left(0_{1 \times 9}, \frac{L_{4}}{2}+O(\mu), 0, O\left(\frac{1}{\chi}\right)\right)_{12 \times 1}^{T}, \\
l_{i i i}=-l_{i i i^{\prime}} & \left.=\left(0_{1 \times 8} ; O\left(\frac{\mu}{\chi^{2}}\right),-\frac{1+O(\mu)}{\chi L_{4}}, O\left(\frac{\mu}{\chi}\right),-\frac{1}{2}\right)_{1 \times 12}\right) .
\end{aligned}
$$

(b.3) The $O(1)$ blocks in $N_{1}, N_{3}$ and $N_{5}$ have exact estimates as follows:

$$
\begin{aligned}
& \left(N_{1}\right)_{44} \simeq\left[\begin{array}{cc}
1-\frac{\tilde{L}_{4, j}^{2}}{2\left(\tilde{L}_{4, j}^{2}+\widetilde{G}_{4, j}^{2}\right)} & -\frac{\tilde{L}_{4, j}}{2} \\
\frac{\tilde{L}_{4, j}^{3}}{2\left(\tilde{L}_{4, j}^{2}+\widetilde{G}_{4, j}^{2}\right)^{2}} & 1+\frac{\tilde{L}_{4, j}^{2}}{2\left(\tilde{L}_{4, j}^{2}+\widetilde{G}_{4, j}^{2}\right)}
\end{array}\right], \\
& \left(N_{3}\right)_{44} \simeq\left[\begin{array}{cc}
\frac{1}{2}-\frac{\tilde{L}_{4, j}}{2} \\
\frac{3}{2 \tilde{L}_{4, j}} \quad \frac{1}{2}
\end{array}\right], \\
& \left(N_{5}\right)_{44} \simeq\left[\begin{array}{cc}
1+\frac{\hat{L}_{4, j}^{2}}{2\left(\hat{L}_{4}^{2}+\widehat{G}_{4, j}^{2}\right)} & -\frac{\hat{L}_{4, j}}{2} \\
\frac{\hat{L}_{4, j}^{3}}{2\left(\hat{L}_{4}^{2}+\widehat{G}_{4, j}^{2}\right)^{2}} & 1-\frac{\hat{L}_{4, j}^{2}}{2\left(\hat{L}_{4, j}^{2}+\widehat{G}_{4, j}^{2}\right)}
\end{array}\right] .
\end{aligned}
$$


where the notation $\simeq$ means up to $O(\mu)$ relative error.

(b.4) Finally, the derivative of the renormalization map is

$$
d \mathcal{R}=\operatorname{diag}\left\{\sqrt{\lambda}, 1,-\sqrt{\lambda},-1 ; \lambda\left[\begin{array}{cc}
1 & 0 \\
0 & -1
\end{array}\right] \operatorname{Rot}(\beta),\left[\begin{array}{cc}
1 & 0 \\
0 & -1
\end{array}\right] \frac{\operatorname{Rot}(\beta)}{\sqrt{\lambda}} ;-\sqrt{\lambda},-1\right\} .
$$

In part (a.1) of the proposition, each of the five matrices is a product of three matrices. For the matrices (I), (III) and (V), we use the formula for the derivative of the Poincaré map (see equation (7.1) and §8). The matrices $N_{1}, N_{3}$ and $N_{5}$ are solutions of the variational equations, and the two remaining matrices are boundary contributions coming from the fact that different orbits take different time to travel between two consecutive sections. For (II), we first convert from Delaunay variables to Cartesian variables in the right, then we use $L \cdot R^{-1}$ to convert $\left(x_{3}, v_{3} ; x_{1}, v_{1} ; x_{4}, v_{4}\right)^{R} \rightarrow$ $\left(x_{3}, v_{3} ; x_{1}, v_{1} ; x_{4}, v_{4}\right)^{L}$, and finally we convert from Cartesian in the left to Delaunay variables. The matrix (IV) is similar but in the opposite direction.

The plan of the proof of Proposition 5.2 is as follows.

- In $\S 6$ and 7 , we write down the equations of motion, the variational equations and estimate their solutions. This gives us the matrices $N_{1}, N_{3}$ and $N_{5}$ in Proposition 5.2.

- In $\S 8$, we study the boundary contribution to the derivative of the Poincare map. We get all the $u$ 's and $l$ 's with various sub- and superscripts in (I), (III) and (V). Together with $N_{1}, N_{3}$ and $N_{5}$, the estimates of the boundary contributions complete the estimates of (I), (III) and (V).

- In $\S 9$, we study the transformation of coordinates from the left to the right and that from the right to the left. This gives us the matrices (II) and (IV) stated in Proposition 5.2.

- The derivative of the renormalization map follows immediately from its definition in Definition 2.7.

We now compute the matrices $R \cdot L^{-1}$ and $L \cdot R^{-1}$ based on Definitions 2.1 and 4.1.

Proof of (5.1). To get

$$
R \cdot L^{-1}=\frac{\partial\left(x_{3}, v_{3} ; x_{1}, v_{1} ; x_{4}, v_{4}\right)^{R}}{\partial\left(x_{3}, v_{3} ; x_{1}, v_{1} ; x_{4}, v_{4}\right)^{L}}
$$

we first use (4.2) to compute the matrix

$$
L^{-1}:=\frac{\partial\left(q_{3}, p_{3} ; q_{1}, p_{1} ; q_{4}, p_{4}\right)}{\partial\left(x_{3}, v_{3} ; x_{1}, v_{1} ; x_{4}, v_{4}\right)^{L}},
$$

then we use (2.3) to compute

$$
R:=\frac{\partial\left(x_{3}, v_{3} ; x_{1}, v_{1} ; x_{4}, v_{4}\right)^{R}}{\partial\left(q_{3}, p_{3} ; q_{1}, p_{1} ; q_{4}, p_{4}\right)} .
$$


The composition of the two gives us $R \cdot L^{-1}$. Similarly, we get

$$
L \cdot R^{-1}=\frac{\partial\left(x_{3}, v_{3} ; x_{1}, v_{1} ; x_{4}, v_{4}\right)^{L}}{\partial\left(x_{3}, v_{3} ; x_{1}, v_{1} ; x_{4}, v_{4}\right)^{R}}=\left(R \cdot L^{-1}\right)^{-1} .
$$

\section{Equations of motion, $\mathscr{C}^{0}$ control of the global map}

\subsection{The Hamiltonian equations}

For the Hamiltonians in Lemmas 4.5 and 4.6, we suppress the super- or subscripts $R$ and $L$ to express the Hamiltonian in one single expression in Delaunay coordinates:

$$
H\left(L_{3}, \ell_{3}, G_{3}, g_{3} ; x_{1}, v_{1} ; L_{4}, \ell_{4}, G_{4}, g_{4}\right)=E_{1}-\frac{m_{3} k_{3}^{2}}{2 L_{3}^{2}}+\frac{m_{4} k_{4}^{2}}{2 L_{4}^{2}}+U
$$

where

$$
E_{1}=\frac{\left|v_{1}\right|^{2}}{2 m_{1}}-\frac{k_{1}}{\left|x_{1}\right|} .
$$

Next, we perform the energy reduction to get rid of the variables $L_{4}$ and $\ell_{4}$.

We solve for $L_{4}$ using energy conservation. Suppose the total energy of the system is zero; we get

$$
\frac{1}{L_{4}^{2}}=\frac{m_{3} k_{3}^{2}}{m_{4} k_{4}^{2} L_{3}^{2}}\left(1-\frac{2 L_{3}^{2}}{m_{3} k_{3}^{2}}\left(E_{1}+U\right)\right)
$$

and hence

$$
L_{4}=L_{3} \frac{m_{4}^{1 / 2} k_{4}}{m_{3}^{1 / 2} k_{3}}\left(1+\frac{L_{3}^{2}}{m_{3} k_{3}^{2}}\left(E_{1}+U\right)+\text { h.o.t. }\right),
$$

where the higher-order terms (h.o.t.) are in $E_{1}+U$. We treat $\ell_{4}$ as the new time. So we divide the Hamiltonian equations by the equation

$$
\frac{d \ell_{4}}{d t}=-\frac{m_{4} k_{4}^{2}}{L_{4}^{3}}+\frac{\partial U}{\partial L_{4}},
$$

whose reciprocal is

$$
\frac{d t}{d \ell_{4}}=-\frac{L_{4}^{3}}{m_{4} k_{4}^{2}}\left(1+\frac{L_{4}^{3}}{m_{4} k_{4}^{2}} \frac{\partial U}{\partial L_{4}}+O\left(U^{2}\right)\right) .
$$

Eliminating $L_{4}$ using (6.1), we get

$$
\begin{aligned}
\frac{d t}{d \ell_{4}} & =-\frac{\left(m_{4}^{1 / 2} k_{4} / m_{3}^{1 / 2} k_{3}\right)^{3}}{m_{4} k_{4}^{2}} L_{3}^{3}\left(1+\frac{3 L_{3}^{2}}{m_{3} k_{3}^{2}}\left(E_{1}+U\right)\right)-\frac{\left(m_{4}^{1 / 2} k_{4} / m_{3}^{1 / 2} k_{3}\right)^{6}}{m_{4}^{2} k_{4}^{4}} L_{3}^{6} \frac{\partial U}{\partial L_{4}}+\text { h.o.t. } \\
& =-(1+O(\mu)) L_{3}^{3}\left(1+3(1+O(\mu)) L_{3}^{2}\left(E_{1}+U\right)\right)-(1+O(\mu)) L_{3}^{6} \frac{\partial U}{\partial L_{4}}+\text { h.o.t. }
\end{aligned}
$$

where, in the last equality, we use the fact that $k_{3,4}, m_{3,4}=1+O(\mu)$. 
Now we write the equations of motion as follows:

$$
\left\{\begin{array} { l } 
{ \frac { d L _ { 3 } } { d \ell _ { 4 } } = - \frac { d t } { d \ell _ { 4 } } \frac { \partial U } { \partial \ell _ { 3 } } , } \\
{ \frac { d G _ { 3 } } { d \ell _ { 4 } } = - \frac { d t } { d \ell _ { 4 } } \frac { \partial U } { \partial g _ { 3 } } , } \\
{ \frac { d x _ { 1 } } { d \ell _ { 4 } } = \frac { d t } { d \ell _ { 4 } } \frac { v _ { 1 } } { m _ { 1 } } , } \\
{ \frac { d G _ { 4 } } { d \ell _ { 4 } } = - \frac { d t } { d \ell _ { 4 } } \frac { \partial U } { \partial g _ { 4 } } , }
\end{array} \quad \left\{\begin{array}{l}
\frac{d \ell_{3}}{d \ell_{4}}=\frac{d t}{d \ell_{4}}\left(\frac{m_{3} k_{3}^{2}}{L_{3}^{3}}+\frac{\partial U}{\partial L_{3}}\right), \\
\frac{d g_{3}}{d \ell_{4}}=\frac{d t}{d \ell_{4}}\left(\frac{\partial U}{\partial G_{3}}\right), \\
\frac{d v_{1}}{d \ell_{4}}=-\frac{d t}{d \ell_{4}}\left(\frac{k_{1} x_{1}}{\left|x_{1}\right|^{3}}+\frac{\partial U}{\partial x_{1}}\right), \\
\frac{d g_{4}}{d \ell_{4}}=\frac{d t}{d \ell_{4}}\left(\frac{\partial U}{\partial G_{4}}\right) .
\end{array}\right.\right.
$$

Notation 6.1 . We denote the right-hand side of $(6.3)$ by $\mathcal{F}=\left(\mathcal{F}_{3} ; \mathcal{F}_{1} ; \mathcal{F}_{4}\right)$. Thus, (6.3) takes the form

$$
\frac{d}{d \ell_{4}} \mathcal{V}_{i}=\mathcal{F}_{i}, \quad i=3,1,4
$$

\subsection{Estimates of the Hamiltonian equations}

\subsubsection{Estimates of the positions}

The next step in our analysis is an important a-priori bound.

We make the following standing assumptions. We first introduce a rectangle to which $x_{4}$ and $x_{1}$ are confined.

Definition 6.2 . We let $\mathcal{S}_{\widehat{C}}$ be the strip bounded by two horizontal lines, $x_{\perp}= \pm \widehat{C}$ and two vertical lines $x_{\|}=-2$ and $x_{\|}=-2 \chi$.

\subsubsection{Estimate of the derivatives of the potential}

We make the following standing assumption:

$$
\left|x_{1}\right| \geqslant 0.9 \chi, \quad\left|x_{4}\right| \leqslant 0.8 \chi, \quad\left|x_{3}\right|<2, \quad\left|x_{3}-x_{4}\right|>\delta>0 .
$$

Lemma 6.3. Define

$$
\mathrm{u}\left(\ell_{4}\right)=\frac{1}{\chi^{3}}+\frac{\mu}{\left|\ell_{4}\right|^{3}+1} .
$$

Suppose we have (6.4) for both the left and right cases, and for the right case, in addition

$$
\frac{1}{C}<\frac{\left|x_{4}^{R}\left(\ell_{4}\right)\right|}{\left|\ell_{4}\right|}<C .
$$


(a) Then, we have the following estimates for the first-order derivatives:

$$
\begin{gathered}
\frac{\partial U^{R}}{\partial x_{3}} \lesssim \mathrm{u}\left(\ell_{4}\right), \quad \frac{\partial U^{R}}{\partial x_{4}} \lesssim \frac{1}{\chi^{2}}+\frac{\mu}{\ell_{4}^{4}+1}, \quad \frac{\partial U^{R, L}}{\partial x_{1}} \lesssim \frac{1}{\chi^{2}} \\
\frac{\partial U^{L}}{\partial x_{3}} \lesssim \frac{1}{\chi^{3}}, \quad \frac{\partial U^{L}}{\partial x_{4}} \lesssim \frac{1}{\chi^{2}}
\end{gathered}
$$

(b) the second-order derivatives satisfy the following estimates

$$
\begin{gathered}
\frac{\partial^{2} U^{R}}{\partial x_{3}^{2}} \lesssim \mathrm{u}\left(\ell_{4}\right), \quad \frac{\partial^{2} U^{R}}{\partial x_{3} \partial x_{4}} \lesssim \frac{\mu}{\chi^{4}}+\frac{\mu}{\left|\ell_{4}\right|^{4}+1}, \quad \frac{\partial^{2} U^{R}}{\partial x_{4}^{2}} \lesssim \frac{1}{\chi^{3}}+\frac{\mu}{\left|\ell_{4}\right|^{5}+1}, \\
\frac{\partial^{2} U^{R, L}}{\partial x_{3} \partial x_{1}} \lesssim \frac{1}{\chi^{4}}, \quad \frac{\partial^{2} U^{L}}{\partial x_{3}^{2}} \lesssim \frac{1}{\chi^{3}}, \quad \frac{\partial^{2} U^{L}}{\partial x_{3} \partial x_{4}} \lesssim \frac{\mu}{\chi^{4}}
\end{gathered}
$$

(c) If we assume furthermore that $x_{1} \in \mathcal{S}_{\mu \widehat{C}}$ and $-x_{4}^{L}, x_{4}^{R} \in \mathcal{S}_{\widehat{C}}$, then we have

$$
\frac{\partial^{2} U^{R, L}}{\partial x_{1}^{2}}, \frac{\partial^{2} U^{R, L}}{\partial x_{4} \partial x_{1}}, \frac{\partial^{2} U^{L}}{\partial x_{4}^{2}} \lesssim \frac{\operatorname{Id}_{2}}{\chi^{3}}+\frac{(\chi, 1)^{\otimes 2}}{\chi^{5}} .
$$

Proof. First, let $X=c_{1} x_{1}+c_{4} x_{4}+c_{3} x_{3}$. Then, we have

$$
\frac{\partial}{\partial x_{i}} \frac{1}{|X|}=-c_{i} \frac{X}{|X|^{3}}, \quad \frac{\partial^{2}}{\partial x_{i} \partial x_{j}} \frac{1}{|X|}=c_{i} c_{j}\left(\frac{-\mathrm{Id}_{2}}{|X|^{3}}+3 \frac{X \otimes X}{|X|^{5}}\right) .
$$

This is enough to give us the estimates

$$
\frac{\partial U^{L}}{\partial x_{4}}, \quad \frac{\partial U^{L}}{\partial x_{3}}, \quad \frac{\partial^{2} U^{R, L}}{\partial x_{3} \partial x_{1}}, \quad \frac{\partial^{2} U^{L}}{\partial x_{3}^{2}}, \quad \frac{\partial^{2} U^{L}}{\partial x_{3} \partial x_{4}}, \quad \frac{\partial^{2} U^{R, L}}{\partial x_{4} \partial x_{1}}, \quad \frac{\partial^{2} U^{L}}{\partial x_{4}^{2}} .
$$

Second, for the estimates

$$
\frac{\partial U^{R, L}}{\partial x_{1}} \text { and } \frac{\partial^{2} U^{R, L}}{\partial x_{1}^{2}}
$$

we need to utilize the cancelation due to the Kepler potentials $1 / \mu\left|x_{1}\right|$. To see the cancelation, we next introduce

$$
f(t)=\frac{1}{|a-t b|},
$$

so $1 /|a-b|=f(1)$. Thus, we get $f(1)=f(0)+f^{\prime}(\xi)$ for some $\xi \in[0,1]$, and hence

$$
\frac{1}{|a-b|}=\frac{1}{|a|}-\frac{a-\xi b}{|a-\xi b|^{3}} \cdot b
$$


for some $\xi \in[0,1]$, by the mean-value theorem. From this, we get $O(1 / \chi)$ instead of the $O(1 / \mu \chi)$ estimate for the term

$$
\frac{2 \mu+1}{\mu\left|x_{1}\right|}-\frac{1}{\mu\left|x_{1}+\mu x_{4} /(1+2 \mu)+\mu x_{3} /(1+\mu)\right|} \quad \text { in } U^{R}
$$

and the term

$$
\frac{(\mu+1)^{2}}{\mu\left|x_{1}\right|}-\frac{1}{\mu\left|x_{1}-\mu x_{4} /(1+\mu)+\mu x_{3} /(1+\mu)\right|} \text { in } U^{L} .
$$

To get the first-order derivative $\partial U^{R, L} / \partial x_{1}$, we use the following:

$$
-\frac{a-b}{|a-b|^{3}}=-\frac{a}{|a|^{3}}+\left(-\frac{\mathrm{Id}_{2}}{|a-\xi b|^{3}}+3 \frac{(a-\xi b) \otimes(a-\xi b)}{|a-\xi b|^{5}}\right) \cdot b
$$

for some $\xi \in[0,1]$. Similarly, we get the second-order derivative $\partial^{2} U^{R, L} / \partial x_{1}^{2}$ using

$$
\begin{aligned}
& \left(-\frac{\mathrm{Id}_{2}}{|a-b|^{3}}+3 \frac{(a-b) \otimes(a-b)}{|a-b|^{5}}\right)-\left(-\frac{\mathrm{Id}_{2}}{|a|^{3}}+3 \frac{a \otimes a}{|a|^{5}}\right) \\
& \quad=-3 \frac{\mathrm{Id}_{2}}{|a-\xi b|^{5}}\langle a-\xi b, b\rangle-3 \frac{b \otimes(a-\xi b)+(a-\xi b) \otimes b}{|a-\xi b|^{5}}+15 \frac{(a-\xi b) \otimes(a-\xi b)}{|a-\xi b|^{7}}\langle a-\xi b, b\rangle
\end{aligned}
$$

and the fact that $x_{4}$ and $x_{1}$ are almost parallel, due to the assumptions $x_{1} \in \mathcal{S}_{\mu \widehat{C}}$ and $-x_{4}^{L}, x_{4}^{R} \in \mathcal{S}_{\widehat{C}}$

Finally, for all the remaining estimates, we use the expansion

$$
\frac{1}{|a+b|}=\frac{1}{|a|} \frac{1}{\sqrt{1+z}}=\frac{1}{|a|}\left(1-\frac{1}{2} z+\sum_{n=2}^{\infty} c_{n} z^{n}\right)
$$

where

$$
z=\frac{2\langle a, b\rangle}{|a|^{2}}+\frac{|b|^{2}}{|a|^{2}} .
$$

We apply the expansion to the term

$$
U_{34}:=\frac{1+\mu}{\left|x_{4}\right|}-\left(\frac{1}{\left|x_{4}+\mu x_{3} /(1+\mu)\right|}+\frac{\mu}{\left|x_{3} /(1+\mu)-x_{4}\right|}\right) \quad \text { in } U^{R}
$$

such that

$$
\begin{aligned}
\frac{1}{\left|x_{4}+\mu x_{3} /(1+\mu)\right|} & =\frac{1}{\left|x_{4}\right|}\left(1-\frac{1}{2} z_{1}+\sum_{n=2}^{\infty} c_{n} z_{1}^{n}\right), \\
z_{1} & =\frac{1}{\left|x_{4}\right|^{2}}\left(2 \mu\left\langle\frac{x_{3}}{1+\mu}, x_{4}\right\rangle+\frac{\mu^{2}}{(1+\mu)^{2}}\left|x_{3}\right|^{2}\right)
\end{aligned}
$$


and

$$
\begin{aligned}
\frac{\mu}{\left|x_{3} /(1+\mu)-x_{4}\right|} & =\frac{\mu}{\left|x_{4}\right|}\left(1-\frac{1}{2} z_{2}+\sum_{n=2}^{\infty} c_{n} z_{2}^{n}\right) \\
z_{2} & =\frac{1}{\left|x_{4}\right|^{2}}\left(-2\left\langle x_{4}, \frac{x_{3}}{1+\mu}\right\rangle+\frac{1}{(1+\mu)^{2}}\left|x_{3}\right|^{2}\right) .
\end{aligned}
$$

It can be verified that the $O\left(1 /\left|x_{4}\right|\right)$ and $O\left(1 /\left|x_{4}\right|^{2}\right)$ terms in $U_{34}$ are canceled. So, we get

$$
-U_{34}=\frac{-\mu}{2(1+\mu)} \frac{\left|x_{3}\right|^{2}}{\left|x_{4}\right|^{3}}+\frac{1}{\left|x_{4}\right|} \sum_{n=2}^{\infty} c_{n}\left(z_{1}^{n}+\mu z_{2}^{n}\right),
$$

and we have the estimates

$$
z_{1}=O\left(\frac{\mu}{\left|\ell_{4}\right|+1}\right) \quad \text { and } \quad z_{2}=O\left(\frac{1}{\left|\ell_{4}\right|+1}\right)
$$

The exponential convergence in $z_{i}$ allows us to take derivatives term by term. For instance, we have

$$
-\frac{\partial U_{34}}{\partial x_{3}}=\frac{-\mu}{1+\mu} \frac{x_{3}}{\left|x_{4}\right|^{3}}+\frac{1}{\left|x_{4}\right|}\left(\frac{\partial z_{1}}{\partial x_{3}}\left(\sum_{n=2}^{\infty} n c_{n} z_{1}^{n-1}\right)+\mu \frac{\partial z_{2}}{\partial x_{3}}\left(\sum_{n=2}^{\infty} n c_{n} z_{2}^{n-1}\right)\right)=O\left(\frac{\mu}{\left|\ell_{4}\right|^{3}+1}\right)
$$

using the estimates

$$
\frac{\partial z_{1}}{\partial x_{3}}=O\left(\frac{\mu}{\left|\ell_{4}\right|+1}\right) \quad \text { and } \quad \frac{\partial z_{2}}{\partial x_{3}}=O\left(\frac{1}{\left|\ell_{4}\right|+1}\right)
$$

We apply the same procedure to all the remaining estimates above.

Lemma 6.4. Suppose (6.4) and in addition $\left|v_{1}\right|<C$. Then, on the zero-energy level, we have

$$
E_{4}=-E_{3}+O(\mu), \quad \text { as } \frac{1}{\sqrt{\chi}} \leqslant \mu \rightarrow 0 .
$$

Proof. This lemma follows directly from the total energy conservation. We write the Hamiltonian as $0=E_{1}+E_{3}+E_{4}+U$. We estimate the potential $U=O(\mu)$ by the assumption. Next,

$$
E_{1}=\frac{1}{2 m_{1}}\left|v_{1}\right|^{2}-\frac{k_{1}}{\left|x_{1}\right|}
$$

where $m_{1} \simeq 1 / 2 \mu$ and $k_{1} \simeq 1 / \mu$ in both the left and right cases. This gives us that

$$
E_{1}=O(\mu) .
$$


Lemma 6.5. Suppose we have (6.4).

(a.1) Suppose in the right case in addition that

$$
\frac{1}{C}<\left|G_{3}\right| \leqslant\left|L_{3}\right|<C, \quad \frac{1}{C}<\frac{\left|x_{4}\left(\ell_{4}\right)\right|}{\left|\ell_{4}\right|}<C \quad \text { and } \quad\left|v_{1}\right|<C .
$$

Then,

$$
\left|\frac{d t}{d \ell_{4}}\right| \sim 1 \quad \text { and } \quad \frac{d}{d \ell_{4}}\left(L_{3}, \ell_{3}, G_{3}, g_{3}\right)=(0,-1,0,0)+O\left(\mathrm{u}\left(\ell_{4}\right), \mu, \mathrm{u}\left(\ell_{4}\right), \mathrm{u}\left(\ell_{4}\right)\right) .
$$

(a.2) Suppose in the left case, in addition to (6.4), that

$$
\frac{1}{C}<\left|G_{3}\right| \leqslant\left|L_{3}\right|<C \quad \text { and } \quad\left|v_{1}\right|<C .
$$

Then, we have

$$
\left|\frac{d t}{d \ell_{4}}\right| \sim 1 \quad \text { and } \quad \frac{d}{d \ell_{4}}\left(L_{3}, \ell_{3}, G_{3}, g_{3}\right)=(0,-1,0,0)+O\left(\frac{1}{\chi^{3}}, \mu, \frac{1}{\chi^{3}}, \frac{1}{\chi^{3}}\right) .
$$

(b.1) Suppose in the right case, in addition to (6.4), that

$$
\frac{1}{C}<\left|L_{3}\right|<C, \quad \frac{1}{C}<\frac{\left|x_{4}\left(\ell_{4}\right)\right|}{\left|\ell_{4}\right|}<C, \quad\left|v_{1}\right|<C, \quad\left|G_{4}\right|<C, \quad x_{4} \in \mathcal{S}_{\widehat{C}}, \quad x_{1} \in \mathcal{S}_{\mu \widehat{C}} .
$$

Then,

$$
\frac{d}{d \ell_{4}}\left(G_{4}, g_{4}\right)=\left(\mathrm{v}\left(\ell_{4}\right)\right)_{1 \times 2}
$$

where

$$
\mathrm{v}\left(\ell_{4}\right):=\frac{1}{\chi^{2}}+\frac{\mu}{\left|\ell_{4}\right|^{3}+1} .
$$

(b.2) Suppose in the left case, in addition to (6.4), that

$$
\frac{1}{C}<\left|L_{3}\right|<C, \quad\left|G_{4}\right|<C, \quad\left|v_{1}\right|<C, \quad-x_{4} \in \mathcal{S}_{\widehat{C}}, \quad x_{1} \in \mathcal{S}_{\mu \widehat{C}} .
$$

Then,

$$
\frac{d}{d \ell_{4}}\left(G_{4}, g_{4}\right)=O\left(\frac{1}{\chi^{2}}\right)_{1 \times 2} .
$$

(c.1) In both the right and the left cases we have

$$
\frac{d x_{1}}{d t}=\frac{v_{1}}{m_{1}} \quad \text { and } \quad \frac{d v_{1}}{d t}=-\frac{k_{1} x_{1}}{\left|x_{1}\right|^{3}}+\text { h.o.t. }=O\left(\frac{1}{\mu \chi^{2}}\right) .
$$

(c.2) If we assume, in addition, that $x_{4}^{R},-x_{4}^{L} \in \mathcal{S}_{\widehat{C}}$ and $x_{1} \in \mathcal{S}_{\mu \widehat{C}}$, then we have

$$
\frac{d x_{1}}{d t}=\frac{v_{1}}{m_{1}} \quad \text { and } \quad \frac{d v_{1}}{d t}=-\frac{k_{1} x_{1}}{\left|x_{1}\right|^{3}}+\text { h.o.t. }=O\left(\frac{1}{\mu \chi^{2}}, \frac{1}{\chi^{3}}\right) .
$$


Proof. We first prove parts (a.1) and (a.2). We apply (6.2) and the assumptions to conclude that

$$
\frac{1}{C} \leqslant\left|\frac{d t}{d \ell_{4}}\right| \leqslant C
$$

Next, we consider the Hamiltonian equation (6.3). We have

$$
\frac{\partial U}{\partial \mathcal{V}_{3}}=\frac{\partial U}{\partial x_{3}} \frac{\partial x_{3}}{\partial \mathcal{V}_{3}}
$$

where $\mathcal{V}_{3}=\left(L_{3}, \ell_{3}, G_{3}, g_{3}\right)$. Because of the boundedness of $L_{3}$, we get that $\left|\partial x_{3} / \partial \mathcal{V}_{3}\right|<C$. Now parts (a.1) and (a.2) are proved by applying Lemma 6.3 (a).

Parts (c.1) and (c.2) follow directly from the Hamiltonian equation. In fact, the estimates for $d v_{1} / d t$ are given by the Kepler motion.

We next prove parts (b.1) and (b.2). We need the estimates

$$
\frac{\partial x_{4}}{\partial g_{4}} \cdot x_{4}=0 \text { and } \quad \frac{\partial x_{4}}{\partial G_{4}} \cdot x_{4}=O\left(\ell_{4}\right)
$$

from part (c) of Lemma A.3 in Appendix A. For example in the right case in (4.3), we consider the derivative of the term

$$
\frac{\partial}{\partial G_{4}} \frac{1}{\mu\left|x_{1}+\mu x_{4} /(1+2 \mu)+\mu x_{3} /(1+\mu)\right|}=\frac{\left(x_{1}+\mu x_{4} /(1+2 \mu)+\mu x_{3} /(1+\mu)\right)}{(1+2 \mu)\left|x_{1}+\mu x_{4} /(1+2 \mu)+\mu x_{3} /(1+\mu)\right|^{3}} \cdot \frac{\partial x_{4}}{\partial G_{4}} .
$$

We claim that the above expression is $O\left(1 / \chi^{2}\right)$. Indeed, the denominator is of order $\chi^{3}$. The main contributions to the numerator come from $\left\langle x_{4}, \partial x_{4} / \partial G_{4}\right\rangle$, which is $O\left(\ell_{4}\right)$, due to part (c) of Lemma A.3, and from $\left\langle x_{1}, \partial x_{4} / \partial G_{4}\right\rangle$. To estimate the later product, we write

$$
x_{1}=\frac{\left|x_{1}\right|}{\left|x_{4}\right|}(\cos \alpha) x_{4}+\left|x_{1}\right|(\sin \alpha) \mathbf{e}
$$

where $\alpha=\angle\left(x_{4}, x_{1}\right)$ and $\mathbf{e}$ is the unit vector perpendicular to $x_{4}$. We note that the assumptions $x_{4} \in \mathcal{S}_{\widehat{C}}$ and $x_{1} \in \mathcal{S}_{\mu \widehat{C}}$, imply $\alpha=O\left(1 / \ell_{4}\right)$. This gives

$$
\left\langle x_{1}, \frac{\partial x_{4}}{\partial G_{4}}\right\rangle=O\left(\frac{\left|x_{1}\right|}{\left|x_{4}\right|}\right)\left\langle x_{4}, \frac{\partial x_{4}}{\partial G_{4}}\right\rangle+\left|x_{1}\right| O(\alpha) O\left(\left|\frac{\partial x_{4}}{\partial G_{4}}\right|\right)=O(\chi),
$$

where the last estimate comes from Lemma A.3 (c).

The other derivatives are estimated similarly and result in the estimates of the lemma. In particular, the $O\left(\mu /\left(\left|\ell_{4}\right|^{3}+1\right)\right)$ part in our bound for $\mathcal{F}_{4}$ comes from differentiating the terms in $U^{R}$ which do not contain $x_{1}$. This bound is obtained by multiplying the $O\left(\mu /\left(\left|\ell_{4}\right|^{4}+1\right)\right)$ term in the estimate of $\partial U^{R} / \partial x_{4}$ in part (a) of Lemma 6.3 by the $O\left(\ell_{4}\right)$ bound on $\partial x_{4} / \partial G_{4}$ from Lemma A.3 (c). 
In the next lemma, we show that the assumption AG, which is only on the initial and final conditions, gives control of the dynamics of $x_{1}, v_{1}, x_{3}$ and $v_{3}$ for all time.

Lemma 6.6. Assume $\mathbf{A G}$ and in addition $\left|x_{4}\right|<0.8 \chi$ for all time $t \in[0, T], T \leqslant 10 \chi$, when $\mathbb{G}$ is defined.

Then, as $1 / \chi \ll \mu \rightarrow 0$, we have that

(a) $L_{3}(t)-L_{3}(0)=O(\mu), G_{3}(t)-G_{3}(0)=O(\mu)$ and $g_{3}(t)-g_{3}(0)=O(\mu), t \in[0, T]$;

(b) $\left|x_{4}^{R, L}\right|=\left(L_{3}^{2}(0)+O(\mu)+o_{\left|\ell_{4}\right| \rightarrow \infty}(1)\right)\left|\ell_{4}^{R, L}\right|$;

(c) for all $t$ defining piece $(\mathrm{I}), x_{1, \perp}^{R}(t)=O(\mu), x_{1, \|}^{R}(t)-x_{1, \|}^{R}(0)=O(\mu \chi), v_{1, \perp}^{R}(t)=$ $O(1 / \chi)$ and $v_{1, \|}^{R}(t)-v_{1, \|}^{R}(0)=O(1 / \mu \chi)$.

Proof. We first consider the piece of orbit going from the section $\left\{x_{4, \|}^{R}=-2\right\}$ to the section $\left\{x_{4, \|}^{R}=-\frac{1}{2} \chi\right\}$ that we called piece (I). Let $[0, \tau]$ be the maximal time interval such that

$$
\left|\frac{G_{3}(t)}{G_{3}(0)}\right|,\left|\frac{L_{3}(t)}{L_{3}(0)}\right| \in\left[\frac{3}{4}, \frac{4}{3}\right], \quad 2\left|G_{4}\left(\ell_{4}^{*}\right)\right|+1, \quad\left|x_{1}^{R}(t)\right| \geqslant 0.95 \chi \quad \text { and } \quad\left|v_{1}^{R}(t)\right| \leqslant C_{0}^{\prime}+1 .
$$

During time $[0, \tau]$, we have (6.4) satisfied with the help of the additional assumption $\left|x_{4}\right|<0.8 \chi$.

We always have $\left|x_{4}\right| \geqslant 2$, since $x_{4}$ is to the left of the section $\left\{x_{4}=-2\right\}$. So, we get $L_{4}(t)=L_{3}(t)+O(\mu)$ for $t \in[0, \tau]$ using Lemma 6.4 and the bound on $\left|v_{1}^{R}(t)\right|$ in $(6.5)$. Then, using formula (A.5) and

$$
e_{4}=\sqrt{1+\frac{G_{4}^{2}}{L_{4}^{2}}}
$$

we find

$$
\begin{aligned}
\left|x_{4}\right| & =\frac{1}{m_{4} k_{4}} L_{4} \sqrt{L_{4}^{2}\left(\cosh u-e_{4}\right)^{2}+G_{4}^{2} \sinh ^{2} u} \\
& =\frac{1}{m_{4} k_{4}} L_{4} \sqrt{L_{4}^{2}\left(\cosh ^{2} u-2 e_{4} \cosh u+e_{4}^{2}\right)+\left(L_{4}^{2} e_{4}^{2}-L_{4}^{2}\right) \sinh ^{2} u} \\
& =\frac{1}{m_{4} k_{4}} L_{4}^{2} \sqrt{1-2 e_{4} \cosh u+e_{4}^{2}+e_{4}^{2} \sinh ^{2} u} \\
& =\frac{1}{m_{4} k_{4}} L_{4}^{2} \sqrt{1-2 e_{4} \cosh u+e_{4}^{2}+e_{4}^{2}\left(\cosh ^{2} u-1\right)} \\
& =\frac{1}{m_{4} k_{4}} L_{4}^{2} \sqrt{\left(1-e_{4} \cosh u\right)^{2}} \\
& =\frac{1}{m_{4} k_{4}} L_{4}^{2}\left(e_{4} \cosh u-1\right)
\end{aligned}
$$

We always have $e_{4} \geqslant 1$, so we get $|\ell-u| \geqslant|\sinh u| \geqslant \frac{1}{2}\left(e^{|u|}-1\right)$ from (A.4), so that $u=o(\ell)$ as $|\ell| \rightarrow \infty$. Continuing (6.6), we have

$$
e_{4} \cosh u \simeq e_{4}|\sinh u|=|\ell-u|=(1+o(1))|\ell| .
$$


So, we obtain

$$
\left|x_{4}\right|=L_{4}^{2}(1+o(1))\left|\ell_{4}\right|, \quad \text { as }\left|\ell_{4}\right| \rightarrow \infty .
$$

By assumption (6.5), we get that

$$
\frac{1}{C}<\frac{\left|x_{4}\left(\ell_{4}\right)\right|}{\left|\ell_{4}\right|}<C
$$

for some constant $C$ for the time interval $[0, \tau]$. So, Lemma 6.5 (a.1) is applicable. Over time $O(\chi)$, we get

$$
L_{3}(t)-L_{3}(0)=O(\mu), \quad G_{3}(t)-G_{3}(0)=O(\mu) \quad \text { and } \quad g_{3}(t)-g_{3}(0)=O(\mu) .
$$

From the Hamiltonian equation, we get

$$
\dot{x}_{1}=\frac{1}{m_{1}} v_{1} \quad \text { and } \quad \dot{v}_{1}=\frac{x_{1}}{\mu\left|x_{1}\right|^{3}}+O\left(\frac{1}{\chi^{2}}\right),
$$

where the $O\left(1 / \chi^{2}\right)$ estimate is from Lemma 6.3 (a). We get that the assumptions on $x_{1}$ and $v_{1}$ in (6.5) are satisfied over time $O(\chi)$. This proves the estimate in item (a) for piece (I). For the estimate in item (a) for pieces (III) and (V), in order to repeat the above argument, we only need to show that $v_{1}$ is bounded, so that Lemma 6.4 applies. From equation (5.1), we get

$$
x_{1}^{L}=\frac{1}{1+\mu} x_{1}^{R}+\frac{2 \mu}{1+2 \mu} x_{4}^{R} \quad \text { and } \quad v_{1}^{L}=\frac{1+\mu}{1+2 \mu} v_{1}^{R}+v_{4}^{R} .
$$

Since we have $L_{4}^{R}(t)=L_{3}(t)+O(\mu)=L_{3}(0)+O(\mu)$ on the section $\left\{x_{4, \|}^{R}=-\frac{1}{2} \chi\right\}$, we get $v_{4}^{R}$ hence $v_{1}^{L}$ is bounded on the section. Now we can repeat the previous case (I) argument to establish the estimate in item (a) for piece (III). Similarly for piece (V).

We next work on item (c) for piece (I) only. We assume $\tau$ is the maximal time such that the following holds:

$$
-1.2 \chi \leqslant x_{1, \|}^{R}(t) \leqslant-0.95 \chi, \quad\left|x_{1, \perp}^{R}(t)\right| \leqslant 1 \quad \text { and } \quad\left|v_{1}^{R}(t)\right| \leqslant C_{0}^{\prime}+1 .
$$

On the time interval $[0, \tau]$, we have

$$
\dot{v}_{1, \perp}^{R}=O\left(\frac{1}{\mu \chi^{3}}+\frac{1}{\chi^{2}}\right)
$$

from (6.8), hence the oscillation of $v_{1, \perp}^{R}$ is bounded by $O\left(1 / \mu \chi^{2}+1 / \chi\right)$ and the oscillation of $x_{1, \perp}^{R}$ is $O(\mu)$ using the equation $\dot{x}_{1, \perp}^{R}=v_{1, \perp}^{R} / m_{1}$ and $m_{1} \sim 1 / \mu$. Therefore, on the time interval $[0, \tau]$, we always have $\left|x_{1, \perp}^{R}\right| \leqslant 1$, and obtain the estimate $x_{1, \perp}^{R}=O(\mu)$. Similarly, we have

$$
x_{1, \|}^{R}(t)-x_{1, \|}^{R}(0)=O(\mu \chi), \quad v_{1, \perp}^{R}(t)=O\left(\frac{1}{\chi}\right) \quad \text { and } \quad v_{1, \|}^{R}(t)-v_{1, \|}^{R}(0)=O\left(\frac{1}{\mu \chi}\right) .
$$

This implies that the assumption (6.10) holds for the entire piece (I) and we have proved item (c). 


\subsection{Justification of the assumptions of Lemma 6.5}

We demonstrate that the orbits satisfying AG satisfy the assumptions of Lemma 6.5. In AG we make assumptions on the initial and final values of $x_{4}$ and $v_{4}$. However, in the assumptions of Lemma 6.5, we require that the orbit of $x_{4}$ to be bounded in $\mathcal{S}_{\widehat{C}}$.

Lemma 6.7. Assume AG for an orbit defined on the time interval $[0, T]$ such that $x_{4, \|}^{R}(0)=x_{4, \|}^{R}(T)=-2$ and $Q_{4}$ turns around $Q_{1}$ once in the sense of Definition 1.1. Then, there exist constants $\widehat{C}$ and $\mu_{0}$ such that, for $\mu \leqslant \mu_{0}$, we have

$$
\left|G_{4}^{L, R}(t)\right|<\widehat{C}, \quad x_{4}^{R},-x_{4}^{L} \in \mathcal{S}_{\widehat{C}} \quad \text { and } \quad x_{1}^{R, L} \in \mathcal{S}_{\mu \widehat{C}} \quad \text { for all } t \in[0, T] .
$$

To prove this result, we first need the following sublemma.

Sublemma 6.8. Given small $\tilde{\theta}>0$ there exist $\mu_{0}$ and $\chi_{0}$ such that, under the assumptions of Lemma 6.7 if $\mu \leqslant \mu_{0}$ and $\chi \geqslant \chi_{0}$ for all $t \in[0, T]$, then

(a) for all $t$ when the orbit is moving to the right of the sections $\left\{x_{4, \|}^{R}=\frac{1}{2} \chi\right\}$ and $\left\{x_{4, \|}^{L}=-\frac{1}{2} \chi\right\}$, we have

$$
\left|\pi-\theta_{4}^{+}(t)\right|<\tilde{\theta} \quad \text { and } \quad\left|\theta_{4}^{-}(t)\right|<\tilde{\theta},
$$

where $\theta_{4}^{+}$(resp. $\left.\theta_{4}^{-}\right)$is the angle of the outgoing (resp. incoming) asymptote of $x_{4}$ (see Notation 2.5);

(b) for all $t$ when the orbit is moving to the left of the sections $\left\{x_{4, \|}^{R}=\frac{1}{2} \chi\right\}$ and $\left\{x_{4, \|}^{L}=-\frac{1}{2} \chi\right\}$, we have $\left|\theta_{4}(t)-\pi\right|<\tilde{\theta}$ for the piece with $u<0$ and $\left|\theta_{4}(t)\right|<\tilde{\theta}$ for the piece with $u>0$. (See Appendix A.2 for the convention of $u$.)

Proof. Pick a large $D$ and let $\tau^{*}$ be the first time when $\left|x_{4}^{R}\left(\tau^{*}\right)\right|=D$, and let $\bar{\tau}$ be the first time when the orbit intersects the section $\left\{x_{4, \|}^{R}=-\frac{1}{2} \chi\right\}$. It is enough to consider below the times $t \geqslant \tau^{*}$. Indeed, $\theta_{4}^{+}$changes by $O(D \mu)$ on the time segment $\left[0, \tau^{*}\right]$, since we have $\theta_{4}^{+}=\pi+g_{4}+\arctan \left(G_{4} / L_{4}\right)$ by $(2.6)$, hence $\dot{\theta}_{4}^{+}=O(\mu)$ by the Hamiltonian equation, and we know that $\tau^{*}$ and $D$ are constants independent of $\mu$. Next,

$$
\theta^{+}\left(\tau^{*}\right)=\arctan \left(\frac{v_{4, \perp}^{R}}{v_{4, \|}^{R}}\right)\left(\tau^{*}\right)+o_{D \rightarrow \infty}(1) .
$$

To fix our idea we suppose that

$$
\tilde{\theta} \leqslant\left|\arctan \left(\frac{v_{4, \perp}^{R}}{v_{4, \|}^{R}}\right)\left(\tau^{*}\right)\right| \leqslant \frac{\pi}{4} .
$$

This implies that $v_{4}$ has a horizontal component that is bounded away from zero, therefore it takes time $O(\chi)$ to travel between two consecutive sections. Under this assumption, we get that $\left|x_{4}\right| \leqslant\left(\frac{1}{2} \sqrt{2}+O(\mu)\right) \chi \leqslant 0.8 \chi$, so that the assumptions of Lemma 6.6 are satisfied. 
Let $\tau^{\dagger}$ be the first time when $\left|v_{4}^{R}\left(\tau^{\dagger}\right)-v_{4}^{R}\left(\tau^{*}\right)\right|>0.01$. For $t \leqslant \min \left(\bar{\tau}, \tau^{\dagger}\right)$, we have

$$
D+c\left(t-\tau^{*}\right)<\left|x_{4}^{R}(t)\right|<D+C\left(t-\tau^{*}\right) .
$$

On the other hand, the Hamiltonian equations give

$$
\dot{v}_{4}^{R}=-(1+O(\mu)) \frac{x_{4}^{R}+O\left(\mu x_{3}\right)}{\left|x_{4}^{R}\right|^{3}}+O\left(\frac{x_{4}^{R}}{\chi^{3}}\right)
$$

where $x_{3}$ is bounded by Lemma $6.6(\mathrm{a})$ :

$$
\left|v_{4}^{R}(t)-v_{4}^{R}\left(\tau^{*}\right)\right| \leqslant 1.1 \int_{\tau^{*}}^{t} \frac{1}{\left|D+c\left(s-\tau^{*}\right)\right|^{2}}+O\left(\frac{D+C\left(s-\tau^{*}\right)}{\chi^{3}}\right) d s=\frac{1.1}{c D}+O\left(\frac{t^{2}}{\chi^{3}}\right) .
$$

Thus, the oscillation of $v_{4}^{R}$ is smaller than $2 / c D$ if $t \leqslant \tau^{\dagger}$ and $t=O(\chi)$. It follows that $\bar{\tau}=O(\chi)$ and $\tau^{\dagger}>\bar{\tau}$.

Next, we change the coordinates to the left variables. From (5.1), we get that

$$
x_{4}^{L}=-x_{1}^{R}+\frac{1+\mu}{1+2 \mu} x_{4}^{R} \quad \text { and } \quad v_{4}^{L}=-\frac{2 \mu}{1+2 \mu} v_{1}^{R}+\frac{1}{1+\mu} v_{4}^{R},
$$

from which we obtain, on the section $\left\{x_{4, \|}^{R}=-\frac{1}{2} \chi\right\}$ that

$$
\left|\arctan \left(\frac{v_{4, \perp}^{L}}{v_{4, \|}^{L}}\right)(\bar{\tau})-\arctan \left(\frac{v_{4, \perp}^{R}}{v_{4, \|}^{R}}\right)\left(\tau^{*}\right)\right| \leqslant \frac{3}{c^{2} D}+O(\mu) \quad \text { and } \quad\left|x_{4, \perp}^{L}(\bar{\tau})\right| \geqslant \frac{\chi}{2} \frac{2}{3} \tilde{\theta}
$$

by choosing $D$ large such that $4 / c^{2} D<\frac{1}{3} \tilde{\theta}$. We apply a similar estimate to the left piece of orbit to show that for the orbit between the two sections $\left\{x_{4, \|}^{R}=-\frac{1}{2} \chi\right\}$ and $\left\{x_{4, \|}^{L}=D\right\}$ the motion of $x_{4}^{L}$ is almost linear with the estimates

$$
\left|\arctan \left(\frac{v_{4, \perp}^{L}}{v_{4, \|}^{L}}\right)\right|>\frac{\tilde{\theta}}{3} \quad \text { and } \quad\left|x_{4, \perp}^{L}\right| \geqslant \frac{\chi \tilde{\theta}}{3}
$$

when arriving at the section $\left\{x_{4, \|}^{L}=D\right\}$.

On the other hand, by definition we have $x_{4}^{L}=q_{4}-q_{1}=Q_{4}-Q_{1}$. In order to have a returning orbit to the section $\left\{x_{4, \|}^{R}=-2\right\}$, the two bodies $Q_{4}$ and $Q_{1}$ have to have a close encounter. This contradicts our estimate of $x_{4}^{L}$ at the end of the previous paragraph. This proves that the slope of the initial outgoing asymptote satisfies $\left|\theta_{4}^{+}-\pi\right|<\tilde{\theta}$. Similarly, we get that the final incoming asymptote satisfies $\left|\theta_{4}^{-}\right|<\tilde{\theta}$ by repeating the above argument with the time reversed. For the estimate in part (b), we apply the same argument above treating the orbits as starting from a neighborhood of $Q_{1}$ moving towards $Q_{2}$. 
Proof of Lemma 6.7. The proof is a refinement of that of Sublemma 6.8 with the same general idea.

The fact that $x_{1}^{R} \in \mathcal{S}_{\mu \widehat{C}}$ is given by Lemma 6.6 (c).

Step 1. (Boundedness of $G_{4}^{R, L}$.)

Without any assumption on $G_{4}$, we have that

$$
\dot{G}_{4}=\frac{\partial U}{\partial x_{4}} \cdot \frac{\partial x_{4}}{\partial g_{4}}
$$

is $O(1 / \chi)$ in the left case and is $O\left(1 / \chi+\mu / \ell_{4}^{3}+1\right)$ in the right case, directly from the estimate of $\partial U / \partial x_{4}$ in Lemma 6.3 , the bound on $L_{3}$, and the fact that $\left|\partial x_{4} / \partial g_{4}\right|=\left|x_{4}\right|$. This implies that the oscillation of $G_{4}$ is $O(1)$ over time $O(\chi)$. By Sublemma $6.8(\mathrm{~b})$, we see that in the left case the slope of asymptotes of $x_{4}^{L}$ is bounded by $\tilde{\theta}$, so $\left|G_{4} / L_{4}\right| \leqslant 2 \tilde{\theta}$. Next, by Lemmas 6.6 and 6.4 , we get that $1 / C<L_{4}<C$. Therefore, $G_{4}^{L}=O(1)$ when $Q_{4}$ comes close to $Q_{1}$. We also assumed that $G_{4}^{R}=O(1)$ on the section $\left\{x_{4, \|}^{R}=-2\right\}$. We get $G_{4}^{L}, G_{4}^{R}=O(1)$ for all the time when they are defined, in particular, when evaluated on the sections $\left\{x_{4, \|}^{R}=-\frac{1}{2} \chi\right\}$ and $\left\{x_{4, \|}^{L}=\frac{1}{2} \chi\right\}$.

Step 2. (Estimate of $x_{4, \perp}^{R}$ and $v_{4, \perp}^{R}$.)

We use (6.12) to get the relation for angular momentum

$$
\begin{aligned}
G_{4}^{L} & =v_{4}^{L} \times x_{4}^{L} \\
& =\left(\frac{1}{1+\mu} v_{4}^{R}-\frac{2 \mu}{1+2 \mu} v_{1}^{R}\right) \times\left(\frac{x_{4}^{R}(1+\mu)}{1+2 \mu}-x_{1}^{R}\right) \\
& =\frac{G_{4}^{R}}{1+2 \mu}-\frac{1}{1+\mu} v_{4}^{R} \times x_{1}^{R}-\frac{2 \mu(1+\mu)}{(1+2 \mu)^{2}} v_{1}^{R} \times x_{4}^{R}+\frac{2 \mu}{1+2 \mu} v_{1}^{R} \times x_{1}^{R} .
\end{aligned}
$$

Using the estimates on $v_{1}^{R}=O(1,1 / \chi), x_{1, \|}^{R} \leqslant-\chi$ and $x_{1, \perp}^{R}=O(\mu)$ from Lemma 6.6 (c), we get that, on the section $\left\{x_{4, \|}^{R}=-\frac{1}{2} \chi\right\}$,

$$
O(1)=G_{4}^{L}-\frac{G_{4}^{R}}{1+2 \mu}=(1+O(\mu))\left[v_{4, \perp}^{R} \chi+O(\mu)\right]+O(\mu)\left[O(1)+x_{4, \perp}^{R}\right]+O(\mu)[O(1)]
$$

This implies that

$$
v_{4, \perp}^{R} \chi=O(1)+O(\mu) x_{4, \perp}^{R} .
$$

Next, we have

$$
O(1)=G_{4}^{R}=v_{4, \perp}^{R} x_{4, \|}^{R}-x_{4, \perp}^{R} v_{4, \|}^{R}=-v_{4, \perp}^{R} \frac{\chi}{2}-x_{4, \perp}^{R} v_{4, \|}^{R} .
$$


Substituting (6.14) into (6.15) and using the lower bound on $v_{4, \|}^{R}$, we get $x_{4, \perp}^{R}=O(1)$. We next substitute the $x_{4, \perp}^{R}$ estimate back into (6.14) to get $v_{4, \perp}^{R}=O(1 / \chi)$. We then obtain $x_{4, \perp}^{L}=O(1)$ and $v_{4, \perp}^{L}=O(1 / \chi)$ using (6.12). Remember that these estimates are only established so far on the sections $\left\{x_{4, \|}^{R}=-\frac{\chi}{2}\right\}$ and $\left\{x_{4, \|}^{L}=\frac{\chi}{2}\right\}$.

Step 3. (Bounding the right piece of the orbit $x_{4}^{R}$.)

We next bound the orbit between the sections $\left\{x_{4, \|}^{R}=-D\right\}$ and $\left\{x_{4, \|}^{R}=-\frac{1}{2} \chi\right\}$ for some large constant $D$ independent of $\chi, \mu$. Suppose the orbit intersects the section $\left\{x_{4, \|}^{R}=-\frac{1}{2} \chi\right\}$ at time $t_{0}$ and the section $\left\{x_{4, \|}^{R}=-D\right\}$ at time $t_{1}$. We have $\left|x_{4, \perp}\left(t_{1}\right)\right| \leqslant C$ for some constant $C$ due to the continuity of the flow and the boundedness of the initial conditions on the section $\left\{x_{4, \|}^{R}=-2\right\}$ as assumed. We have

$$
x_{4, \perp}(t)=x_{4, \perp}\left(t_{0}\right)+v_{4, \perp}\left(t_{0}\right)\left(t-t_{0}\right)+\int_{t_{0}}^{t} \int_{t_{0}}^{u} \ddot{x}_{4, \perp}(s) d s d u
$$

where, for piece (I), we have $t_{1}<s<t_{0}$ and

$$
\ddot{x}_{4, \perp}(s)=O\left(\frac{x_{4, \perp}(s)}{\left|x_{4}(s)\right|^{3}}+\frac{\left|x_{4}\right|}{\chi^{3}}+\frac{\mu\left|x_{3}\right|}{\left|x_{4}(s)\right|^{3}}\right)=O\left(\frac{x_{4, \perp}(s)+\mu}{\left(\frac{1}{2} \chi+\left(s-t_{0}\right)\right)^{3}}+\frac{\left|x_{4}\right|}{\chi^{3}}\right) .
$$

By Step 2, we have $x_{4, \perp}\left(t_{0}\right)=O(1)$ and $v_{4, \perp}\left(t_{0}\right)=O(1 / \chi)$. We bound the double integral of the term $\left|x_{4}\right| / \chi^{3}$ in $\ddot{x}_{4, \perp}$ by a constant. So, we get

$$
\left|x_{4, \perp}(t)\right| \leqslant C+C \sup _{t_{0}<s<t_{1}}\left|x_{4, \perp}(s)\right| \int_{t_{0}}^{t} \int_{t_{0}}^{u} \frac{1}{\left(\frac{1}{2} \chi+\left(s-t_{0}\right)\right)^{3}} d s d u \leqslant C+\frac{C}{D} \sup _{t_{0}<s<t_{1}}\left|x_{4, \perp}(s)\right| .
$$

Choosing $D>C$, this shows that $x_{4}^{R} \in \mathcal{S}_{\widehat{C}}$ for some large $\widehat{C}$ for the piece of orbit in consideration. For piece $(\mathrm{V}), t_{0}<s<t_{1}$ and the denominator of the integrand becomes $\left(\frac{1}{2} \chi-\left(s-t_{0}\right)\right)^{3}$.

Step 4. (Bounding the left piece of the orbit $x_{4}^{L}$ and the returning orbit.)

We have $x_{4, \perp}^{L}=O(1)$ on the section $\left\{x_{4, \|}^{L}=D\right\}$, by Sublemma $6.8(\mathrm{~b})$. We apply the same argument as in Step 3 to both halves of the left piece between the sections $\left\{x_{4, \|}^{L}=D\right\}$ and $\left\{x_{4, \|}^{R}=-\frac{1}{2} \chi\right\}$. This shows that $-x_{4}^{L} \in \mathcal{S}_{\widehat{C}}$ for the piece of orbit in consideration. The fact that $x_{1}^{L} \in \mathcal{S}_{\mu \widehat{C}}$ follows from the $v_{4, \perp}, x_{4, \perp}$ estimate in Step 2, Lemma 6.6 (c) and equations (6.8) and (6.9).

Remark 6.9. From the proof, we see that Lemma 6.7 still holds if, instead of $\mathbf{A G}$, we assume (AG.3), (AG.1) and $\left|x_{4, \perp}^{R}(0)\right|<2$ and $x_{4, \|}^{R}(0)=-2$ for the initial condition and $x_{4}^{L}(T)=0$ for the final condition. The final condition implies a collision between $Q_{1}$ and $Q_{4}$. The crucial ingredient in the proof is that $Q_{1}$ must come close to $Q_{4}$ in order to have a return orbit. 
All the assumptions of Lemma 6.5 are implied by AG due to Lemma 6.7 and Lemma 6.6, so we have the following.

Corollary 6.10. Assume AG. Then,

(a) in the right case, we have

$$
\mathcal{F}^{R}=\left(0,1,0_{1 \times 8}\right)+O\left(\mathrm{u}\left(\ell_{4}\right), \mu, \mathrm{u}\left(\ell_{4}\right), \mathrm{u}\left(\ell_{4}\right) ; \mu, \frac{\mu}{\chi}, \frac{1}{\mu \chi^{2}}, \frac{1}{\chi^{3}} ; \mathrm{v}\left(\ell_{4}\right), \mathrm{v}\left(\ell_{4}\right)\right) ;
$$

(b) in the left case, we have

$$
\mathcal{F}^{L}=\left(0,1,0_{1 \times 8}\right)+O\left(\frac{1}{\chi^{3}}, \mu, \frac{1}{\chi^{3}}, \frac{1}{\chi^{3}} ; \mu, \frac{\mu}{\chi}, \frac{1}{\mu \chi^{2}}, \frac{1}{\chi^{3}} ; \frac{1}{\chi^{2}}, \frac{1}{\chi^{2}}\right) .
$$

Lemma 6.11. Assume AG. Then, we have

(a) when $x_{4}$ is moving to the right of the sections $\left\{x_{4, \|}^{R}=-\frac{1}{2} \chi\right\}$ and $\left\{x_{4, \|}^{L}=\frac{1}{2} \chi\right\}$, we have

$$
\tan g_{4}=-\operatorname{sign}(u) \frac{G_{4}}{L_{4}}+O\left(\frac{\mu}{\left|\ell_{4}\right|^{2}+1}+\frac{1}{\chi}\right), \quad \text { as }\left|\ell_{4}\right| \rightarrow \infty \text { and } \frac{1}{\chi} \ll \mu \rightarrow 0 ;
$$

(b) when $x_{4}$ is moving to the left of the sections $\left\{x_{4, \|}^{R}=-\frac{1}{2} \chi\right\}$ and $\left\{x_{4, \|}^{L}=\frac{1}{2} \chi\right\}$, then

$$
G_{4}, g_{4}=O\left(\frac{1}{\chi}\right) \quad \text { as } \frac{1}{\chi} \ll \mu \rightarrow 0 \text {. }
$$

Proof. The proof is to integrate the estimates of $d\left(G_{4}, g_{4}\right) / d \ell_{4}$ in Lemma 6.5 (b.1) and (b.2).

Step 1. We prove part (b). Integrating the Hamiltonian equation for $G_{4}^{L}$ and $g_{4}^{L}$ in Lemma 6.5 (b.2) starting from $\ell_{4}=0$, we get

$$
\left(G_{4}^{L}, g_{4}^{L}\right)\left(\ell_{4}\right)=\left(G_{4}^{L}, g_{4}^{L}\right)(0)+O\left(\frac{1}{\chi}\right)
$$

when arriving at the sections $\left\{x_{4, \|}^{R}=-\frac{1}{2} \chi\right\}$ and $\left\{x_{4, \|}^{L}=\frac{1}{2} \chi\right\}$. To conclude part (b), we need to show that the initial conditions $G_{4}^{L}(0)$ and $g_{4}^{L}(0)$ are bounded by $O(1 / \chi)$. Using (A.6) (we omit the superscript $L$ and subscript 4), we have on the sections $\left\{x_{4, \|}^{R}=-\frac{1}{2} \chi\right\}$ and $\left\{x_{4, \|}^{L}=\frac{1}{2} \chi\right\}$

$$
\begin{aligned}
x_{4, \perp} & =\frac{1}{m k}\left(\sin g L^{2}(\cosh u-e)+\cos g L G \sinh u\right) \\
& =\frac{1}{m k}\left(\sin g(0) L^{2}(\cosh u-e)+\cos g(0) L G(0) \sinh u\right)+O(1) .
\end{aligned}
$$


Note that this holds for both large positive and large negative $u$, and that on both sections $\cosh u$ and $|\sinh u|$ are of order $\chi$. By Lemma 6.7 , we have $-x_{4}^{L} \in \mathcal{S}_{\widehat{C}}$, which shows that $\left|x_{4, \perp}^{L}\right| \leqslant 2 \widehat{C}$ on the sections $\left\{x_{4, \|}^{R}=-\frac{1}{2} \chi\right\}$ and $\left\{x_{4, \|}^{L}=\frac{1}{2} \chi\right\}$. Next, we apply Lemmas 6.4 and 6.6 to get that $1 / C<\left|L_{4}\right|<C$. Next, arguing as in Step 1 of Lemma 6.7, we have that $\left|G_{4}\right|<C$, and this implies that $e_{4}<C$. This implies that $|g(0)|,|G(0)|=O(1 / \chi)$.

Step 2. Then, we use the matrix $R \cdot L^{-1}$ in Proposition 5.2 to convert the left variables to the right to obtain $v_{4}^{R}=O(\mu) v_{1}^{L} \pm(1+O(\mu)) v_{4}^{L}$. From Step 1 and (A.6), we get that the slope of $v_{4}^{L}$ is

$$
g_{4}^{L}-\arctan \left(\frac{G_{4}^{L}}{L_{4}^{L}}\right)+O\left(\frac{1}{\chi^{2}}\right)=O\left(\frac{1}{\chi}\right),
$$

and from assumption (AG.1) and part (c.1) of Lemma 6.5 that the slope of $v_{1}^{L}$ is $O(1 / \chi)$. So the slope of $v_{4}^{R}$ is

$$
g_{4}^{R}-\arctan \left(\frac{G_{4}^{R}}{L_{4}^{R}}\right)+O\left(\frac{1}{\chi^{2}}\right)=O\left(\frac{1}{\chi}\right)
$$

on the sections $\left\{x_{4, \|}^{R}=-\frac{1}{2} \chi\right\}$ and $\left\{x_{4, \|}^{L}=\frac{1}{2} \chi\right\}$ due to (A.5).

Step 3. To prove part $(a)$, we use the $O(1 / \chi)$ estimates of the slope of $v_{4}^{R}$ in Step 2 as our initial condition. We get that the oscillation of $G_{4}^{R}, g_{4}^{R}$ is

$$
O\left(\frac{\mu}{\left|\ell_{4}\right|^{2}+1}+\frac{1}{\chi}\right)
$$

from $\ell_{4}=O(\chi)$ to $\ell_{4}$ by integrating the $d G_{4}^{R} / d \ell_{4}$ and $d g_{4}^{R} / d \ell_{4}$ estimates in Lemma 6.5.

\subsection{Collision exclusion}

The following lemma excludes the possibility of collisions between $Q_{1}$ and $Q_{4}$.

LEMma 6.12. If we assume (AG.3) and (AG.1), and $\left|x_{4, \perp}^{R}(0)\right|<2$ and $x_{4, \|}^{R}(0)=-2$ for the initial condition and $x_{4}^{L}(T)=0$ for the final condition (collision between $Q_{4}$ and $\left.Q_{1}\right)$, then there is an orbit bouncing back from the $Q_{1}-Q_{4}$ collision lying entirely in a strip $\mathcal{S}_{\widehat{C}}$ for some constant $\widehat{C}$. Moreover, we have $\bar{G}_{4}^{R}+G_{4}^{R}=O(\mu)$ when evaluated on the section $\left\{x_{4, \|}^{R}=-2\right\}$, where $G_{4}^{R}$ and $\bar{G}_{4}^{R}$ are the angular momentum of $\left(x_{4}, v_{4}\right)^{R}$ before and after the application of the global map, respectively.

Proof. By Remark 6.9, the assumption implies the assumptions of Lemma 6.6 according to Lemma 6.7, so we can use the conclusions of Lemma 6.6. 
Suppose we have a collision. We compare the bouncing back orbit (subscript "out") with the time reversal of the incoming orbit (subscript "in"). We will show that the orbits are close and so the values of $G_{4}$ will be close when evaluated on the section $\left\{x_{4, \|}^{R}=-2\right\}$.

Step 1. (Comparing orbits to the left of the line $x_{4, \|}^{R}=-\frac{1}{2} \chi$.)

For the collisional orbit and the bouncing back orbit to the left of the section $x_{4, \|}^{R}=-\frac{1}{2} \chi$, the value $\ell_{4}=0$ corresponds to the collision. When $\ell_{4}=0$, all the values of $\left(L_{3}, g_{3}, x_{1}, G_{4}, g_{4}\right)$ are the same for the two orbits and the variables $\left(G_{3}, \ell_{3}, v_{1}\right)$ have opposite signs for the two orbits. Moreover, since the variables $\mathbf{Y}=\left(G_{4}, g_{4}\right)$ are constants of motion when the potential $U$ is neglected, we get that for small $\left|\ell_{4}\right|>0$ the values of $\mathbf{Y}$ for the two orbits will stay close.

Let $\mathbf{F}$ be the right-hand side of the corresponding Hamiltonian equations (6.3) for $\mathbf{Y}$. We denote $\delta \mathbf{Y}=\mathbf{Y}_{\text {in }}-\mathbf{Y}_{\text {out }}$. Taking the difference of the Hamiltonian equations for $\mathbf{Y}_{\text {in }}$ and $\mathbf{Y}_{\text {out }}$, we have

$$
\frac{d}{d \ell_{4}} \delta \mathbf{Y}=\frac{\partial \mathbf{F}}{\partial \mathbf{Y}}\left(\mathbf{Y}_{\text {in }}, \widehat{\mathbf{Y}}_{\text {in }}\right) \delta \mathbf{Y}+O\left(|\delta \mathbf{Y}|^{2}\right)+\left[\mathbf{F}\left(\mathbf{Y}_{\text {in }}, \widehat{\mathbf{Y}}_{\text {in }}\right)-\mathbf{F}\left(\mathbf{Y}_{\text {in }}, \widehat{\mathbf{Y}}_{\text {out }}\right)\right]
$$

where we denote $\widehat{\mathbf{Y}}:=\left(L_{3}, \ell_{3}, G_{3}, g_{3} ; x_{1}, v_{1}\right)$ and in the bracketed term we fix $\mathbf{Y}_{\text {in }}$, since the difference $\mathbf{Y}_{\text {in }}-\mathbf{Y}_{\text {out }}$ is considered in the $\delta \mathbf{Y}$ and $|\delta \mathbf{Y}|^{2}$ terms.

We trace the orbit back to the section $\left\{x_{4, \|}^{R}=-\frac{1}{2} \chi\right\}$. During the $O(\chi)$ time, the oscillation of $x_{1}$ and $v_{1}$ are estimated as

$$
\left(\delta x_{1}, \delta v_{1}\right)=\left(O(\mu \chi), O(\mu) ; O(1), O\left(\frac{1}{\chi}\right)\right)
$$

from Lemma 6.5 (c.2), as well as the sign change of the initial condition.

The term $\left[\mathbf{F}\left(\mathbf{Y}_{\text {in }}, \widehat{\mathbf{Y}}_{\text {in }}\right)-\mathbf{F}\left(\mathbf{Y}_{\text {in }}, \widehat{\mathbf{Y}}_{\text {out }}\right)\right]$ can be estimated as $O\left(1 / \chi^{3}+\mu / \chi^{2}\right)$, where the estimate $1 / \chi^{3}$ is given by $\partial \mathbf{F} / \partial x_{3}$, due to different values of $\ell_{3}$ for the two orbits, and the estimate

$$
O\left(\frac{\mu}{\chi^{2}}\right)=\frac{\partial \mathbf{F}}{\partial x_{1}} \delta x_{1}
$$

is due to different $x_{1}$ for the two orbits (see Lemma 6.3). The variable $v_{1}$ enters through $d t / d \ell_{4}$ in (6.3) and causes a difference in $\mathbf{F}$ that is much smaller than the above two cases for $\ell_{3}$ and $x_{1}$.

We denote by $\ell_{4}^{\mathrm{f}}$ the time when the time reversed incoming orbit hits $\left\{x_{4, \|}^{R}=-\frac{1}{2} \chi\right\}$. Note the initial condition $\left(\mathbf{Y}_{\text {in }}-\mathbf{Y}_{\text {out }}\right)(0)=0$ and that the fundamental solution of the variational equation

$$
Z^{\prime}=\frac{\partial \mathbf{F}}{\partial \mathbf{Y}} Z
$$


is $O(1)$ (the fundamental solution is given by the matrix $N_{3}$ in Proposition 5.2. Here we pick only the rows and columns corresponding to variables in $\mathbf{Y}$. In fact, we have the estimate

$$
\frac{\partial \mathbf{F}}{\partial \mathbf{Y}}=O\left(\frac{1}{\chi}\right)
$$

in Lemma 7.3 (b) below). Since we have by DuHamel's principle that

$$
\int_{0}^{\ell_{4}^{\mathrm{f}}} O(1) O\left(\frac{\mu}{\chi^{2}}+\frac{1}{\chi^{3}}\right) d \ell_{4}=O\left(\frac{\mu}{\chi}\right)
$$

the Gronwall inequality gives $\mathbf{Y}_{\text {in }}-\mathbf{Y}_{\text {out }}=O(\mu / \chi)$ at time $\ell_{4}^{\mathrm{f}}$.

Step 2. (Cartesian coordinates.)

We already had in Step 1 the estimate of $\delta\left(x_{1}^{L}, v_{1}^{L}\right)$. We need to control the change of $\left(x_{4}, v_{4}\right)^{L}$ as well. We have

$$
\delta\left(x_{4}, v_{4}\right)^{L}=\left[\delta L_{4} \frac{\partial}{\partial L_{4}}+\delta G_{4} \frac{\partial}{\partial G_{4}}+\delta g_{4} \frac{\partial}{\partial g_{4}}\right]^{L}\left(x_{4}, v_{4}\right)^{L} .
$$

Note that here we do not have $\delta \ell_{4} \partial / \partial \ell_{4}$, since we have the same $\ell_{4}=\ell_{4}^{\mathrm{f}}$ for the two orbits, so $\delta \ell_{4}=0$. We use Lemma A.3 in the appendix to get the partial derivatives

$$
\frac{\partial v_{4}}{\partial *}=O(1) \quad \text { and } \quad \frac{\partial x_{4}}{\partial *}=O(\chi), \quad *=L_{4}, G_{4}, g_{4}
$$

and, in particular,

$$
\frac{\partial v_{4, \perp}}{\partial L_{4}}=C \frac{G_{4}}{L_{4}\left(G_{4}^{2}+L_{4}^{2}\right)}=O\left(\frac{1}{\chi}\right) \quad \text { and } \quad \frac{\partial x_{4, \perp}}{\partial L_{4}}=C \frac{G_{4} L_{4}^{2} \ell_{4}}{\left(G_{4}^{2}+L_{4}^{2}\right)}=O(1)
$$

since $G_{4}=O(1 / \chi)$ by Lemma $6.11(\mathrm{~b})$.

The estimates for $\left(\delta G_{4}, \delta g_{4}\right)^{L}=O(\mu / \chi)$ are obtained in Step 1. The estimate of $\delta L_{4}$ is obtained from $(6.1)$ and

$$
\delta L_{4}=\frac{\partial L_{4}}{\partial \mathbf{Y}} \delta \mathbf{Y}+L_{4}\left(\mathbf{Y}_{\text {in }}, \hat{\mathbf{Y}}_{\text {in }}\right)-L_{4}\left(\mathbf{Y}_{\text {in }}, \hat{\mathbf{Y}}_{\text {out }}\right)
$$

We have

$$
\frac{\partial L_{4}}{\partial \mathbf{Y}}=O(1), \quad \delta \mathbf{Y}=O(\mu / \chi), \quad L_{4}\left(\mathbf{Y}_{\text {in }}, \hat{\mathbf{Y}}_{\text {in }}\right)-L_{4}\left(\mathbf{Y}_{\text {in }}, \hat{\mathbf{Y}}_{\text {out }}\right)=O(\mu)
$$

where the main contribution to the last $\mu$ estimate is given by

$$
\frac{\partial L_{4}}{\partial v_{1}}=O(\mu) \quad \text { and } \quad \delta v_{1}=O(1)
$$


So, we get $\delta L_{4}=O(\mu)$.

This implies $\delta\left(x_{4}, v_{4}\right)^{L}=O(\mu \chi, \mu ; \mu, \mu / \chi)$. We also have $\left(x_{4}, v_{4}\right)^{L}=O(\chi, 1 ; 1,1 / \chi)$ and $\left(x_{1}, v_{1}\right)^{L}=O(\chi, \mu, 1,1 / \chi)$ at time $\ell_{4}^{\mathrm{f}}$ (see Step 2 of Lemma 6.7 for the estimate of $x_{4, \perp}$ and $\left.v_{4, \perp}\right)$.

Step 3. (Comparing angular momenta.)

Using the relation

$$
\begin{aligned}
G_{4}^{R} & =v_{4}^{R} \times x_{4}^{R} \\
& =\left(\frac{1+\mu}{1+2 \mu} v_{4}^{L}+\frac{2 \mu}{1+2 \mu} v_{1}^{L}\right) \times\left(\frac{x_{4}^{L}}{1+\mu}+x_{1}^{L}\right) \\
& =\frac{G_{4}^{L}}{1+2 \mu}+\frac{1+\mu}{1+2 \mu} v_{4}^{L} \times x_{1}^{L}+\frac{2 \mu}{(1+2 \mu)(1+\mu)} v_{1}^{L} \times x_{4}^{L}+\frac{2 \mu}{1+2 \mu} v_{1}^{L} \times x_{1}^{L},
\end{aligned}
$$

and the results of Step 2 we get $\delta G_{4}^{R}=O(\mu)$ at time $\ell_{4}^{\mathrm{f}}$.

Step 4. (Oscillation of $G_{4}^{R}$ to the right of $\left\{x_{4, \|}^{R}=-\frac{1}{2} \chi\right\}$.)

Now, we consider the right pieces of orbits. For the collisional orbit the oscillations of $G_{4}^{R}$ are $O(\mu)$ by integrating the estimate in Corollary 6.10. In order to apply the same estimate to the bouncing back orbit, we need to show that the bouncing back orbit lies in the strip $\mathcal{S}_{\widehat{C}}$.

We first get

$$
\delta\left(x_{4}, v_{4}\right)^{R}=O\left(\mu \chi, \mu ; \mu, \frac{\mu}{\chi}\right)
$$

using $R \cdot L^{-1}$ from (5.1) of Proposition 5.2, and the estimates of $\delta\left(x_{4}, v_{4}, x_{1}, v_{1}\right)^{L}$ above. At the time $\ell_{4}^{\mathrm{f}}$, the collisional orbit is on the section $\left\{x_{4, \|}^{R}=-\frac{1}{2} \chi\right\}$, but the bouncing back orbit might be $O(\mu \chi)$ distance away. Tracing the bouncing back orbit over time $O(\mu \chi)$, so that it is also on the section $\left\{x_{4, \|}^{R}=-\frac{1}{2} \chi\right\}$, since

$$
\dot{v}_{4}=-\frac{k_{4} x_{4}}{\left|x_{4}\right|^{3}}+\text { h.o.t., }
$$

we see that $v_{4}$ gains a new oscillation $O(\mu / \chi)$. Comparing the two orbits on the section $\left\{x_{4, \|}^{R}=-\frac{1}{2} \chi\right\}$, we get $\delta\left(x_{4}, v_{4}\right)^{R}=O(0, \mu ; \mu, \mu / \chi)$. Applying (6.16), we see that the $x_{4, \perp}^{R}$ components for the two orbits stay $O(D \mu)$-close to each other when traveling between $\left\{x_{4, \|}^{R}=-\frac{1}{2} \chi\right\}$ and $\left\{x_{4, \|}^{R}=-D\right\}$ for some large $D$ independent of $\mu$ and $\chi$. This shows that the bouncing back orbit also lies in the strip $\mathcal{S}_{\widehat{C}}$ since the collisional orbit does.

Now we apply Corollary 6.10 to get that the oscillation of $G_{4}^{R}$ for the bouncing back orbit is also $O(\mu)$ when traveling between the sections $\left\{x_{4, \|}^{R}=-\frac{1}{2} \chi\right\}$ and $\left\{x_{4, \|}^{R}=-2\right\}$. 
Steps 1-4 show that difference between the angular momenta of the reversed incoming orbit and the bouncing back orbit is $O(\mu)$. Without the time reversal we have

$$
\bar{G}_{4}^{R}+G_{4}^{R}=O(\mu)
$$

as claimed.

The possibility of collision between $Q_{4}$ and $Q_{1}$ is excluded, since in Gerver's construction, $\bar{G}_{4}+G_{4}$ is always bounded away from zero independent of $\mu$. Now, we exclude the possibility of collisions between $Q_{3}$ and $Q_{4}$. Note that $Q_{3}$ and $Q_{4}$ have two potential collision points corresponding to two intersections of the ellipse of $Q_{3}$ and the branch of the hyperbola utilized by $Q_{4}$. See Figures 1 and 2. Now, it follows from Lemma 10.2 (b) that $Q_{3}$ and $Q_{4}$ do not collide near the intersection where they have the close encounter. We need also to rule out the collision near the second intersection point. This was done by Gerver in [G2]. Namely, he shows that the times for $Q_{3}$ and $Q_{4}$ to move from one crossing point to the other are different. As a result, if $Q_{3}$ and $Q_{4}$ come to the correct intersection points nearly simultaneously, they do not collide at the wrong points. In the setting of our paper $(\mu>0)$, the travel times for $Q_{3}$ and $Q_{4}$ to move from one crossing point to the other are $O(\mu)$ perturbations of that computed in [G2]. So, it is impossible to have a collision at a wrong intersection point.

\subsection{Proofs of Lemmas 2.11 and 2.12}

In this section, we prove of Lemmas 2.11 and 2.12. Now, we prove Lemma 2.11.

Proof of Lemma 2.11. We first prove part (c). We first get that

$$
x_{1, \|}(t)=(1+O(\mu)) x_{1, \|}(0)=(1+O(\mu)) \chi
$$

by integrating part (c) of Lemma 6.5 for $t \in[0,100 \chi]$. Next, by Lemmas 6.4 and 6.6, we get that $E_{4}(t)=-E_{3}(t)+O(\mu)=\frac{1}{2}+O(\delta+\mu)$ for $t \in[0,100 \chi]$. This implies that $\left|v_{4}\right| \geqslant$ $1-O(\delta+\mu)$. Next, by Sublemma 6.8 , we get that $\left|v_{4, \|}\right|>\frac{3}{4}$ by choosing $\delta, \mu$ and $\tilde{\theta}$ small. So, we get that the total return time $T \leqslant 2(1+O(\mu)) \chi / \frac{3}{4}<3 \chi(<100 \chi)$.

Since AG implies the assumptions of Lemma 6.5, combined with Lemma 6.6, we get part (a) of Lemma 2.11 from Lemma 6.6, and part (b) from Lemma 6.11 using Notation 2.5.

Now we are ready to prove Lemma 2.12.

Proof of Lemma 2.12. The idea of the proof is to integrate the equations $\dot{x}_{1}$ and $\dot{v}_{1}$ for the pieces (I), (III) and (V), and apply the coordinate changes (II) and (V), to keep track of the change of $x_{1}$ and $v_{1}$. The main idea was sketched in Remark 2.13. 
Step 0. (Preparations.)

We use Lemma 6.5 (c.1) and (c.2) to get that

$$
v_{1}=v_{1}(0)+O\left(\frac{1}{\mu \chi}, \frac{1}{\chi^{2}}\right)
$$

during time $O(\chi)$. It follows from

$$
\frac{d x_{1, \|}}{d t}=\frac{v_{1, \|}}{m_{1}}
$$

that over time $O(\chi)$, the horizontal component $x_{1, \|}$ can move only distance $O(\mu \chi)$. Moreover, the local map takes only $O(1)$ time as $1 / \chi \ll \mu \rightarrow 0$.

Initially, we have angular momentum conservation $G_{1}+G_{3}+G_{4}=0$. Also, from the initial conditions in the assumption (i) and the total energy conservation (Lemma 6.4), we estimate

$$
\left|G_{3}\right|,\left|G_{4}\right| \leqslant 2 C_{0}^{\prime}+1
$$

We get from the definition of angular momentum, Lemma 6.6 (c) and assumption (ii), that

$$
v_{1, \perp}(0)|\leqslant| v_{1, \|} \frac{x_{1, \perp}}{x_{1, \|}}|+| \frac{G_{1}}{x_{1, \|}} \mid \leqslant O\left(\frac{\mu}{\chi}\right)+\frac{2\left(2 C_{0}^{\prime}+1\right)}{\chi} \leqslant \frac{4\left(C_{0}^{\prime}+1\right)}{\chi} .
$$

Step 1. (Piece (I) composed with the local map.)

We integrate the $d v_{1, \|} / d t$ estimates from the section $\left\{x_{4, \|}^{R}=-2, v_{4, \|}^{R}>0\right\}$ to the section $\left\{x_{4, \|}^{R}=-\frac{1}{2} \chi, v_{4, \|}^{R}<0\right\}$ (note that the local map is included). The total traveling time is $<3 \chi$ by Lemma 2.11 (c). In the following we use the notation $a=O_{+}(b)$ if $b>0, a=O(b)$ and $a / b>c>0$ for some constant $c$. Using Lemma 6.5 (c), as $1 / \chi \ll \mu \rightarrow 0$, we have

$$
\begin{gathered}
x_{1, \|}^{R}-x_{1, \|}^{R}(0)=O_{+}(\mu \chi) v_{1, \|}^{R}(0), \quad x_{1, \perp}^{R}-x_{1, \perp}^{R}(0)=O(\mu \chi) v_{1, \perp}^{R}(0)=O(\mu), \\
v_{1, \|}^{R} \in\left[-\bar{c}_{1},-c_{1}\right]+O\left(\frac{1}{\mu \chi}\right), \quad v_{1, \perp}^{R}=O\left(\frac{1}{\chi}\right)
\end{gathered}
$$

on the section $\left\{x_{4, \|}^{R}=-\frac{1}{2} \chi, v_{4, \|}^{R}<0\right\}$. On the same section, we also have

$$
v_{4, \|}^{R}=-\sqrt{2 E_{4}}+O\left(\frac{1}{\chi}\right)=-\sqrt{-2 E_{3}}+O(\mu) \quad \text { and } \quad v_{4, \perp}^{R}=O\left(\frac{1}{\chi}\right)
$$

by Lemmas 6.4 and 6.11, and equation (A.5) (see also Step 2 of the proof of Lemma 6.7). 
Step 2. (Piece (III).)

We use Lemma 6.7 to get $x_{4}^{R} \in \mathcal{S}_{\widehat{C}}$, i.e. $\left|x_{4, \|}^{R}\right| \leqslant 2 \chi$ and $\left|x_{4, \perp}^{R}\right| \leqslant \widehat{C}$. Then, we use (5.1) to get, on the section $\left\{x_{4, \|}^{R}=-\frac{1}{2} \chi, v_{4, \|}^{R}<0\right\}$, that

$$
\begin{aligned}
& x_{1, \|}^{L}=\frac{1}{1+\mu} x_{1, \|}^{R}(0)+O_{+}(\mu \chi) v_{1, \|}^{R}(0)-\frac{\mu \chi}{1+2 \mu}=\frac{1}{1+\mu} x_{1, \|}^{R}(0)-\frac{\mu \chi}{1+2 \mu}-O_{+}(\mu \chi), \\
& x_{1, \perp}^{L}=\frac{1}{1+\mu} x_{1, \perp}^{R}(0)+O(\mu \chi) v_{1, \perp}^{R}(0)+O\left(\frac{\mu}{\chi}\right)=O(\mu), \\
& v_{1, \|}^{L} \in \frac{1+\mu}{1+2 \mu}\left[-\bar{c}_{1},-c_{1}\right]-\sqrt{-2 E_{3}}+O(\mu), \quad v_{1, \perp}^{L}=O\left(\frac{1}{\chi}\right), \quad \text { as } \frac{1}{\chi} \ll \mu \rightarrow 0 .
\end{aligned}
$$

We integrate $d v_{1} / d t$ again over time $O(\chi)$ to get

$$
\begin{aligned}
& x_{1, \|}^{L}=\frac{1}{1+\mu} x_{1, \|}^{R}(0)-\frac{\mu \chi}{1+2 \mu}-O_{+}(\mu \chi), \quad x_{1, \perp}^{L}=O(\mu), \\
& v_{1, \|}^{L} \in \frac{1+\mu}{1+2 \mu}\left[-\bar{c}_{1},-c_{1}\right]-\sqrt{-2 E_{3}}+O(\mu), \quad v_{1, \perp}^{L}=O\left(\frac{1}{\chi}\right), \quad \text { as } \frac{1}{\chi} \ll \mu \rightarrow 0
\end{aligned}
$$

when arriving at the section $\left\{x_{4, \|}^{L}=\frac{\chi}{2}, v_{4, \|}^{L}>0\right\}$ where the $-O_{+}(\mu \chi)$ term in $x_{1, \|}^{L}$ has absorbed a new $-O_{+}(\mu \chi)$ contribution since $v_{1, \|}^{L}<0$. Again it follows from Lemma 6.11 and the energy conservation that

$$
v_{4, \|}^{L}=\sqrt{2 E_{4}}+O\left(\frac{1}{\chi}\right)=\sqrt{-2 E_{3}}+O(\mu) \quad \text { and } \quad v_{4, \perp}^{R}=O\left(\frac{1}{\chi}\right) .
$$

Step 3. (Piece (V).)

We apply (5.1) and $-x_{4}^{L} \in \mathcal{S}_{\widehat{C}}$ (Lemma 6.7) to get that, on the section $\left\{x_{4, \|}^{L}=\frac{1}{2} \chi\right\}$,

$$
\begin{aligned}
x_{1, \|}^{R} & =\frac{1+\mu}{1+2 \mu}\left(\frac{x_{1, \|}^{R}(0)}{1+\mu}-\frac{\mu \chi}{1+2 \mu}\right)-\frac{\mu \chi}{1+2 \mu}-O_{+}(\mu \chi) \\
& =\frac{x_{1, \|}^{R}(0)}{1+2 \mu}-\frac{\mu(2+3 \mu) \chi}{(1+2 \mu)^{2}}-O_{+}(\mu \chi), \\
x_{1, \perp}^{R} & =O(\mu), \quad v_{1, \perp}^{R}=O\left(\frac{1}{\chi}\right), \\
v_{1, \|}^{R} & \in \frac{1}{1+2 \mu}\left[-\bar{c}_{1},-c_{1}\right]-2 \sqrt{-2 E_{3}^{*}}+O(\delta+\mu),
\end{aligned}
$$

as $1 / \chi \ll \mu \rightarrow 0$, where the extra $O(\mu)$ in $v_{1, \|}^{R}$ comes from the oscillation of $E_{3}$ established in Lemma 2.11 (a), and $O(\delta)$ is the deviation of the initial value $E_{3}$ from Gerver's value $E_{3}^{*}$, which is bounded by $C_{3} \delta$. Finally, we get the same estimate as (6.18) when arriving at the section $\left\{x_{4, \|}^{R}=-2, v_{4, \|}^{R}>0\right\}$ with a new $-O_{+}(\mu \chi)$ added to $x_{1, \|}^{R}$. This completes 
one application of $\mathcal{P}$, and hence the proof of part (a). Part (b) is proved in a way similar to Step 1, by composing the local map. The information that we need from $x_{1, \|}^{R}$ is that $x_{1, \|}^{R}<x_{1, \|}^{R}(0)$ after one application of $\mathcal{P}$. Indeed, it follows from the first row of (6.18) and the assumption on $x_{1, \|}^{R}$ that

$$
x_{1, \|}^{R}-x_{1, \|}^{R}(0)=\frac{2 \mu \chi}{1+2 \mu}-\frac{\mu(2+3 \mu) \chi}{(1+2 \mu)^{2}}-O_{+}(\mu \chi)=-O_{+}\left(\mu^{2} \chi\right)-O_{+}(\mu \chi)<0 .
$$

Step 4. (Renormalization.)

One period in Gerver's construction consists of $\mathcal{R} \circ \widetilde{\mathbb{G}} \circ \mathcal{P}^{2}$. We repeat the above procedure to get after $\mathcal{P}^{2}$ (we use double bar for the orbit parameters),

$$
\begin{aligned}
& \overline{\bar{x}}_{1, \|}^{R}-x_{1, \|}^{R}(0)=-O_{+}(\mu \chi)<0, \quad \overline{\bar{x}}_{1, \perp}^{R}=O(\mu), \quad \overline{\bar{v}}_{1, \perp}^{R}=O\left(\frac{1}{\chi}\right), \\
& \overline{\bar{v}}_{1, \|}^{R} \in \frac{1}{(1+2 \mu)^{2}}\left[-\bar{c}_{1},-c_{1}\right]-2 \sqrt{-2 E_{3}^{*}}-2 \sqrt{-2 E_{3}^{* *}}+O(\delta+\mu),
\end{aligned}
$$

as $1 / \chi \ll \mu \ll \delta \rightarrow 0$. The last step is to apply the renormalization $\mathcal{R}$. Let us forget about the rotation by $\beta$ in Definition 2.7 for a moment, and consider only the rescaling. We expect that

$$
\mathcal{R}\left(\overline{\bar{v}}_{1, \|}^{R}\right)=\frac{1}{\sqrt{\lambda}} \overline{\bar{v}}_{1, \|}^{R} \in\left[-\bar{c}_{1},-c_{1}\right]
$$

which is implied by

$$
\overline{\bar{v}}_{1, \|}^{R} \in \frac{1}{(1+2 \mu)^{2}}\left[-\bar{c}_{1},-c_{1}\right]-2 \sqrt{-2 E_{3}^{*}}-2 \sqrt{-2 E_{3}^{* *}}+O(\delta+\mu) \subset \sqrt{\lambda}\left[-\bar{c}_{1},-c_{1}\right],
$$

where $\lambda$ is the renormalization factor in Definition 2.7. This implies

$$
c_{1}+\tilde{c}(\delta+\mu) \leqslant \frac{2}{\sqrt{\lambda}-1}\left(\sqrt{-2 E_{3}^{*}}+\sqrt{-2 E_{3}^{* *}}\right) \leqslant \bar{c}_{1}-\tilde{c}(\delta+\mu)
$$

for some constant $\tilde{c}$ bounding the $O$ in the above estimates. We choose

$$
\bar{c}_{1}=\frac{4}{\sqrt{\lambda}-1}\left(\sqrt{-2 E_{3}^{*}}+\sqrt{-2 E_{3}^{* *}}\right) \quad \text { and } \quad c_{1}=\frac{1}{\sqrt{\lambda}-1}\left(\sqrt{-2 E_{3}^{*}}+\sqrt{-2 E_{3}^{* *}}\right)
$$

so that the above inequality is satisfied uniformly for all sufficiently small $\mu, \delta$ and $1 / \chi$. This completes the proof for $\mathcal{R}\left(\overline{\bar{v}}_{1, \|}^{R}\right)$.

Step 5. (The estimates of $\mathcal{R}\left(x_{1}\right)$.)

This estimate follows by iterating (6.19) twice and applying the renormalization map $\mathcal{R}$. 
Now let us take care of the rotation $\beta$ of $\mathcal{R}\left(\overline{\bar{v}}_{1, \|}\right)$ which is

$$
\arctan \left(\frac{\overline{\bar{x}}_{1, \perp}}{\overline{\bar{x}}_{1, \|}}\right)=O\left(\frac{\mu}{\chi}\right)
$$

by definition. This produces an error of $O(\mu / \chi)$ to $\overline{\bar{v}}_{1, \|}$, which can be absorbed into the $O$ part of the estimate of $\overline{\bar{v}}_{1, \|}$ in (6.20), so that we leave our choice of $c_{1}$ and $\bar{c}_{1}$ unchanged.

If $\widetilde{\mathbb{G}}$ in the definition of $\mathcal{R}$ were the identity, then the rotation $\operatorname{Rot}(\beta)$ would set $x_{1, \perp}$ to zero. Applying $\widetilde{G}$ causes an error $O(\mu / \chi)$ to $x_{1, \perp}$ obtained by integrating the estimate of $\dot{x}_{1, \perp}=O(\mu / \chi)$ over time $O(1)$. Since $\mu / \chi \ll 1 / 2 \sqrt{\lambda \chi}$, we get the estimate for $\mathcal{R}\left(x_{1, \perp}\right)$ in the statement. The $\mathcal{R}\left(x_{1, \|}\right)$ estimate comes from the definition of $\tilde{\chi}=\lambda \chi$ and the cube.

Step 6. (Bounding the angular momentum and vertical component of the velocity $\overline{\bar{v}}_{1, \perp \cdot)}$

After $\mathcal{P}^{2}$ and $\mathcal{R} \circ \widetilde{\mathbb{G}} \circ \mathcal{P}^{2}$, we have angular momentum conservation

$$
\overline{\bar{G}}_{1}+\overline{\bar{G}}_{3}+\overline{\bar{G}}_{4}=0 \quad \text { and } \quad \mathcal{R}\left(\overline{\bar{G}}_{1}\right)+\mathcal{R}\left(\overline{\bar{G}}_{3}\right)+\mathcal{R}\left(\overline{\bar{G}}_{4}\right)=0
$$

After renormalization, $\mathcal{R}\left(\overline{\bar{E}}_{3}\right)$ is now $-\frac{1}{2}+O(1 / \sqrt{\chi})$, and $\left|\overline{\bar{x}}_{4, \perp}\right| \leqslant 2$. The energy conservation shows that $\left|\mathcal{R}\left(\overline{\bar{v}}_{4}\right)\right| \leqslant 1+O(\mu)$, so that we have

$$
\left|\mathcal{R}\left(\overline{\bar{G}}_{3}\right)\right|,\left|\mathcal{R}\left(\overline{\bar{G}}_{4}\right)\right| \leqslant 2 C_{0}^{\prime}+1
$$

From the definition of angular momentum and (6.20), we get that

$$
\left|\mathcal{R}\left(\overline{\bar{v}}_{1, \perp}\right)\right| \leqslant\left|\mathcal{R}\left(\overline{\bar{v}}_{1, \|}^{R}\right) \frac{\mathcal{R}\left(\overline{\bar{x}}_{1, \perp}^{R}\right)}{\mathcal{R}\left(\overline{\bar{x}}_{1, \|}^{R}\right)}\right|+\left|\frac{\mathcal{R}\left(\overline{\bar{G}}_{1}\right)}{\mathcal{R}\left(\overline{\bar{x}}_{1, \|}^{R}\right)}\right| \leqslant O\left(\frac{\mu}{\chi}\right)+\frac{2\left(2 C_{0}^{\prime}+1\right)}{\tilde{\chi}} \leqslant \frac{4\left(C_{0}^{\prime}+1\right)}{\widetilde{\chi}} .
$$

This completes the proof of the $\mathcal{R}\left(\overline{\bar{v}}_{1, \perp}\right)$ estimate in part (c) by defining

$$
C_{1}:=4\left(C_{0}^{\prime}+1\right)
$$

\subsection{Choosing angular momentum: proof of Lemma 2.21}

In this section, we prove Lemma 2.21. We first need two auxiliary results.

Sublemma 6.13. Let $S \subset U_{1}\left(\delta^{\prime}\right)$ and $\tilde{e}_{4}$ be as in part (a) of Lemma 2.21. Then, there exists $\tilde{\ell}_{3}$ such that $\pi_{e_{4}} \mathcal{P}\left(\mathcal{S}\left(\tilde{e}_{4}, \tilde{\ell}_{3}\right)\right)=e_{4}^{* *}$. There are analogous statements for $S \subset U_{2}\left(\delta^{\prime}\right)$ and $S \subset U_{0}\left(\delta^{\prime}\right)$ as in parts (b) and (c) of Lemma 2.21. 
We give the proof of this sublemma immediately after we complete the proof of Lemma 2.21. The next sublemma is easy to prove.

Sublemma 6.14. Let $F$ be a map from $\mathbb{R}^{2}$ to $\mathbb{R}^{2}$ such that

(1) $F\left(a^{*}\right)=b^{*}$ for some $a^{*}, b^{*} \in \mathbb{R}^{2}$;

(2) if $\left|F(z)-b^{*}\right|<R$ for some $R>0$, then $\|d F(z)(X)\| \geqslant \bar{\chi}\|X\|$ for all vectors $X \in$ $T_{z} \mathbb{R}^{2}$ and for some $\bar{\chi}>1$.

Then, for each $b$ such that $\left|b-b^{*}\right|<R$, there exists $z$ such that $|z|<R / \bar{\chi}$ and $F(z)=b$.

With the help of the two sublemmas, we finish the proof of Lemma 2.21.

Proof of Lemma 2.21. We consider part (a) first. Pick a piece of $\delta^{\prime}$-admissible surface $S \subset U_{1}(\delta)$ for $\delta^{\prime}<\delta$.

Choose any $\tilde{e}_{4} \in\left(e_{4}^{*}-\delta^{\prime}+1 / \chi, e_{4}^{*}+\delta^{\prime}-1 / \chi\right)$. By Sublemma 6.13, there exists $\tilde{\ell}_{3}$ such that $\pi_{e_{4}} \mathcal{P}\left(\mathcal{S}\left(\tilde{e}_{4}, \tilde{\ell}_{3}\right)\right)=e_{4}^{* *}$. Let $\ell_{3}^{\prime}=\pi_{\ell_{3}} \mathcal{P}\left(\mathcal{S}\left(\tilde{e}_{4}, \tilde{\ell}_{3}\right)\right)$. Then, $\mathcal{Q}_{1}\left(\tilde{e}_{4}, \tilde{\ell}_{3}\right)=\left(e_{4}^{* *}, \ell_{3}^{\prime}\right)$. Our coordinates allow us to treat $\mathcal{Q}_{1}$ as a map $\mathbb{R} \times \mathbb{T} \rightarrow \mathbb{R} \times \mathbb{T}$. Let $\widetilde{\mathcal{Q}}_{1}: \mathbb{R}^{2} \rightarrow \mathbb{R}^{2}$ be the covering map of $\mathcal{Q}_{1}$. We now apply Sublemma 6.14 , using $a^{*}=\left(\tilde{e}_{4}, \tilde{\ell}_{3}\right), b^{*}=\left(e_{4}^{* *}, \ell_{3}^{\prime}\right), F=\widetilde{\mathcal{Q}}_{1}, \bar{\chi}=c \chi$ (from Lemma 2.17) and letting $R$ be the distance from $b^{*}$ to the boundary of $\mathcal{C}_{2}(\delta)$. This gives us a surjective map, satisfying the expansion condition, from a subset of $\mathcal{C}_{1}\left(\delta^{\prime}\right)$ to the open disk of radius $R$ around $b^{*}$. To extend this map to other parts of $\mathcal{C}_{2}(\delta)$, we can apply Sublemma 6.14 again, using a different $b^{*}$, choosing each $b^{*}$ from a region to which the map has already been extended. Because $\mathcal{C}_{2}(\delta)$ is open and connected, we can eventually extend our surjective map to all of $\mathcal{C}_{2}(\delta)$, although we might have to apply Sublemma 6.14 an infinite number of times. Because of the expansion condition, and the fact that the diameter of $\mathcal{C}_{2}(\delta)$ is $O(1)$, the diameter of the pre-image $V_{1}\left(\tilde{e}_{4}\right)$ is $O(1 / \chi)$. This establishes Lemma 2.21 (a).

Part (b) is similar to part (a). For part (c), we first apply Sublemma 6.13 to find $\tilde{\ell}_{3}$ for each $\tilde{e}_{4}$ such that $\pi_{e_{4}} \mathcal{P}\left(\mathcal{S}\left(\tilde{e}_{4}, \tilde{\ell}_{3}\right)\right)=e_{4}^{* *}$ for a given admissible surface $S \subset U_{0}\left(\delta^{\prime}\right)$. This gives the first statement in part (c). We next introduce the renormalization $\mathcal{R}$ based at the point $\mathcal{S}\left(\tilde{e}_{4}, \tilde{\ell}_{3}\right)$ and obtain $\mathcal{P} \mathcal{R} \widetilde{\mathbb{G}}$ satisfying $\pi_{e_{4}} \mathcal{P} \mathcal{R} \widetilde{\mathbb{G}}\left(\mathcal{S}\left(\tilde{e}_{4}, \tilde{\ell}_{3}\right)\right)=e_{4}^{* *}+O(\mu)$. (We get from $\mathcal{S}\left(\tilde{e}_{4}, \tilde{\ell}_{3}\right)$ to $\mathcal{P}\left(\mathcal{S}\left(\tilde{e}_{4}, \tilde{\ell}_{3}\right)\right)$ by following the Hamiltonian flow, and we get to $\widetilde{\mathbb{G}}\left(\mathcal{S}\left(\tilde{e}_{4}, \tilde{\ell}_{3}\right)\right)$ by following the first part of that same flow. $\mathcal{P R}$ continues the flow on a rotated, rescaled, reflected orbit, but those transformations do not change $e_{4}$ or $\ell_{3}$, so if we continued the flow to the same section as $\mathcal{P}\left(\mathcal{S}\left(\tilde{e}_{4}, \tilde{\ell}_{3}\right)\right)$, but rotated, rescaled and reflected, we would still get $e_{4}^{* *}$. Instead, we continue a distance of $O(1)$ to a new section, mostly because of the rescaling, but also from the rotation, and this changes $e_{4}$ by $O(\mu)$, because of the interaction between $Q_{3}$ and $Q_{4}$.) Because $\delta^{\prime} \gg \mu$, we still have $\mathcal{Q}_{0}\left(\tilde{e}_{4}, \tilde{\ell}_{3}\right) \in \mathcal{C}_{2}\left(\delta^{\prime}\right)$. By Lemma 2.17 and Sublemma 6.14 again, we get a neighborhood $V_{0}\left(\tilde{e}_{4}\right)$ such that $\mathcal{Q}_{0}$ maps $V_{0}\left(\tilde{e}_{4}\right)$ surjectively to $\mathcal{C}_{2}(\delta)$. By Lemma 2.17 , the weakest expansion rate of $d \mathcal{P}$ 
restricted to the cone fields is a constant times $\chi$, hence the diameter of $V_{0}\left(\tilde{e}_{4}\right)$ is $O(1 / \chi)$. Since we have $O(1 / \chi) \ll 1 / \sqrt{\chi}$, we get that $\mathcal{R}$ is well defined in a neighborhood of $V_{0}\left(\tilde{e}_{4}\right)$.

Part (d) is given in Lemma 10.2 (b).

Proof of Sublemma 6.13. The idea is to apply the strong expansion of the Poincaré map in a neighborhood of the collisional orbit studied in Lemma 6.12. Note that Delaunay coordinates regularize double collisions, in the sense that none of the variables blows up at a double collision, so that our estimate of $d \mathbb{G}$ also holds for collisional orbits. We give the proof only for initial conditions on an admissible surface $S \subset U_{1}\left(\delta^{\prime}\right)$. The other cases are similar.

Step 1. We first show that there is a collisional orbit satisfying $x_{4}^{L}(t)=0$ at some time $t$, as $\ell_{3}$ varies.

We apply the local map $\mathbb{L}$ to the admissible surface $S$ with $\tilde{e}_{4}$ fixed. Sublemma 6.8 and its proof shows that, if after the application of the local map we have $\theta_{4}^{+}(0)=\pi-\bar{\theta}$, $0<\bar{\theta}<\left(\tilde{\theta}\right.$ in Lemma 3.1), then the $x_{4, \perp}^{L}$ coordinate is a large positive number of order $\bar{\theta} \chi$ when the orbit hits $\left\{x_{4, \|}^{L}=0\right\}$. Similarly, if $\theta_{4}^{+}(0)=\pi+\bar{\theta}$, then the orbit hits the line $\left\{x_{4, \|}^{L}=0\right\}$, so that its $x_{4, \perp}^{L}$ coordinate is a large negative number. By the intermediatevalue theorem, there has to be an outgoing angle $\theta_{4}^{+}(0)$ leading to a collisional orbit with $x_{4}^{L}=0$. So, it suffices to show that our admissible surface $S$ contains points $\boldsymbol{x}_{1}$ and $\boldsymbol{x}_{2}$ such that $\theta_{4}^{+}\left(\boldsymbol{x}_{1}\right)=\pi-\bar{\theta}$ and $\theta_{4}^{+}\left(\boldsymbol{x}_{2}\right)=\pi+\bar{\theta}$. We have the expression

$$
\theta_{4}^{+}=\pi+\tilde{g}_{4}+\arctan \left(\frac{\widetilde{G}_{4}}{\tilde{L}_{4}}\right)
$$

(see (2.6) for the formula and see Lemma 3.2 for the tilde notation). By direct calculation, we find $d \theta_{4}^{+}=\tilde{L}_{4} \hat{\overline{\mathbf{l}}}$ (see Lemma 3.2 for $\hat{\overline{\mathbf{l}}}$ and Notation 2.5 for $\theta^{+}$). Since $T S \subset \mathcal{K}_{1}$ and the cone $\mathcal{K}_{1}$ is centered at the plane $\operatorname{span}\left(w_{1}, \widetilde{w}\right)$, where $\widetilde{w}=\partial / \partial \ell_{3}$ (Definition 2.16). We get

$$
d \theta^{+} \cdot\left(d \mathbb{L} \frac{\partial}{\partial \ell_{3}}\right)=\tilde{L}_{4} \hat{\overline{\mathbf{l}}}_{1} \cdot\left(\frac{1}{\mu}\left(\widehat{\mathbf{u}}_{1}\left(\hat{\mathbf{l}}_{1} \cdot \widetilde{w}\right)+o(1)\right)+O(1)\right)=\frac{c(\boldsymbol{x})}{\mu},
$$

where $c(\boldsymbol{x}) \neq 0$ by Lemma 3.4 (c). So, it is enough to vary $\ell_{3}$ in a $O(\mu)$ neighborhood of a point whose outgoing asymptote satisfies the assumption of Lemma 3.1 in order to get angles of outgoing asymptotes $\pi \pm \bar{\theta}$. Thus, we get a collisional orbit for some point denoted by $\left(\tilde{e}_{4}, \hat{\ell}_{3}\right)$.

Step 2. We next show that there exists $\ell_{3}$ such that $\pi_{e_{4}}\left(\mathcal{P}\left(\mathcal{S}\left(\tilde{e}_{4}, \ell_{3}\right)\right)\right)$ is close to $e_{4}^{* *}$ for fixed $\tilde{e}_{4}$. Now, the function $\pi_{e_{4}}\left(\mathcal{P}\left(\mathcal{S}\left(\tilde{e}_{4}, \cdot\right)\right)\right)$ is a function of one variable $\ell_{3}$ defined in a neighborhood of $\hat{\ell}_{3}$.

Since $e_{4}=\sqrt{1+\left(G_{4} / L_{4}\right)^{2}}$ is not an injective function of $G_{4}$, we use $G_{4}$ instead of $e_{4}$ and study the function $G_{4}\left(\mathcal{P}\left(\mathcal{S}\left(\tilde{e}_{4}, \ell_{3}\right)\right)\right)$. 
Next, we compute

$$
\begin{aligned}
\frac{d}{d \ell_{3}} G_{4}\left(\mathcal{P}\left(\mathcal{S}\left(\tilde{e}_{4}, \ell_{3}\right)\right)\right) & =d G_{4} d \mathbb{G} d \mathbb{L} \frac{\partial}{\partial \ell_{3}} \\
& =\chi^{2}\left(d G_{4} w_{1}\right) \hat{\overline{\mathbf{l}}} \cdot \cdot\left(d \mathbb{L} \frac{\partial}{\partial \ell_{3}}\right)+O(\chi) \\
& =\bar{c}(\boldsymbol{x}) \frac{\chi^{2}}{\mu}+O(\chi),
\end{aligned}
$$

where $\hat{\overline{\mathbf{l}}}_{1} \cdot\left(d \mathbb{L} \partial / \partial \ell_{3}\right)$ is calculated in Step $1, d G_{4} \widetilde{w}=0$ and $d G_{4} w_{1}=1$. This derivative calculation holds, provided AG and $\mathbf{A L}$ are satisfied, so that we can apply Lemmas 3.1 and 3.2 .

For the collisional orbit, its bouncing back orbit will intersect the section $\left\{x_{4, \|}^{R}=-2\right\}$ at a point that is within $O(\mu)$ distance from the initial point. To see this, we apply to (A.5) the estimate of the difference of $G_{4}$ for two orbits in Lemma 6.12, and the $O(\mu)$ estimate of angle of asymptotes and oscillations of $L_{3}, G_{3}$ and $g_{3}$ in Lemma 2.11 (b).

So, we consider the image of an $\varepsilon$ interval centered at $\hat{\ell}_{3}$ under the map

$$
G_{4}\left(\mathcal{P}\left(\mathcal{S}\left(\tilde{e}_{4}, \cdot\right)\right)\right) \text {. }
$$

By increasing $\varepsilon$ from zero, we see that the assumptions $\mathbf{A G}$ and $\mathbf{A L}$ are all satisfied, provided the returning orbit has $\left|x_{4, \perp}^{R}\right|<C_{0}^{\prime}$ on the section $\left\{x_{4, \|}^{R}=-2\right\}$. Thus, we always have the estimate (6.21), and we can keep increasing $\varepsilon$ until the inequality $\left|x_{4, \perp}^{R}\right|<C_{0}^{\prime}$ is violated.

Thus, it follows from the strong expansion of the map $G_{4}\left(\mathcal{P}\left(\mathcal{S}\left(\tilde{e}_{4}, \cdot\right)\right)\right)$ and Sublemma 6.14 that an $R$-neighborhood of $G_{4}^{* *}$ (corresponding to $e_{4}^{* *}$ ) is covered if $\ell_{3}$ varies in an $\left(R \mu / \bar{c} \chi^{2}\right)$-neighborhood of $\hat{\ell}_{3}$. Then, we use the intermediate-value theorem to find $\tilde{\ell}_{3}$ such that $\pi_{e_{4}} \mathcal{P}\left(\mathcal{S}\left(\tilde{e}_{4}, \tilde{\ell}_{3}\right)\right)=e_{4}^{* *}$. This completes the proof.

\section{The variational equation and its solution}

In this section, we first derive a formula for estimating the derivatives of (I), (III) and (V). This formula will reduce the derivative computation to the fundamental solution of the variational equation and two boundary terms, where the latter takes care of the issue that different orbits might take different time to travel between two consecutive sections. The rest of this section is devoted to estimating the variational equations and their fundamental solutions. This will give the estimates of $N_{1}, N_{5}$ and $M$ in Proposition 5.2.

Let us first recall the notation. We use $\mathcal{V}=\left(\mathcal{V}_{3} ; \mathcal{V}_{1} ; \mathcal{V}_{4}\right)=\left(L_{3}, \ell_{3}, G_{3}, g_{3} ; x_{1}, v_{1} ; G_{4}, g_{4}\right)$ to denote the Delaunay coordinates. We use $\mathcal{X}=\left(\mathcal{X}_{3} ; \mathcal{X}_{1} ; \mathcal{X}_{4}\right)=\left(x_{3}, v_{3} ; x_{1}, v_{1} ; x_{4}, v_{4}\right)$ to denote the Cartesian coordinates. We use $\mathcal{F}=\left(\mathcal{F}_{3}, \mathcal{F}_{1}, \mathcal{F}_{4}\right)$ to denote the right-hand side of the Hamiltonian equation in Delaunay coordinates, i.e. $d \mathcal{V} / d \ell_{4}=\mathcal{F}$. 


\subsection{Derivation of the formula for the boundary contribution}

Suppose that we want to compute the derivative of the Poincaré map between the sections $S^{\mathrm{i}}$ and $S^{\mathrm{f}}$. We use $\mathcal{V}^{\mathrm{i}}$ to denote the values of variables $\mathcal{V}$ restricted to the initial section $S^{\mathrm{i}}$, while $\mathcal{V}^{\mathrm{f}}$ means values of $\mathcal{V}$ on the final section $S^{\mathrm{f}} . \ell_{4}^{\mathrm{i}}$ means the initial time and $\ell_{4}^{\mathrm{f}}$ means the final time. We want to compute the derivative $\mathcal{D}$ of the Poincaré map along the orbit starting from $\left(\mathcal{V}_{*}^{\mathrm{i}}, \ell_{*}^{\mathrm{i}}\right)$ and ending at $\left(\mathcal{V}_{*}^{\mathrm{f}}, \ell_{*}^{\mathrm{f}}\right)$. We have $\mathcal{D}=d F_{3} d F_{2} d F_{1}$, where $F_{1}$ is the Poincaré map between $S^{\mathrm{i}}$ and $\left\{\ell_{4}=\ell_{*}^{\mathrm{i}}\right\}, F_{2}$ is the flow map between the times $\ell_{*}^{\mathrm{i}}$ and $\ell_{*}^{\mathrm{f}}$, and $F_{3}$ is the Poincaré map between $\left\{\ell_{4}=\ell_{*}^{\mathrm{f}}\right\}$ and $S^{\mathrm{f}}$. We have $F_{1}=\Phi\left(\mathcal{V}^{\mathrm{i}}, \ell_{4}\left(\mathcal{V}^{\mathrm{i}}\right), \ell_{*}^{\mathrm{i}}\right)$, where $\Phi(\mathcal{V}, a, b)$ denotes the flow map starting from $\mathcal{V}$ at time $a$ and ending at time $b$. Since

we have

$$
\frac{\partial \Phi}{\partial \mathcal{V}}\left(\mathcal{V}_{*}^{\mathrm{i}}, \ell_{*}^{\mathrm{i}}, \ell_{*}^{\mathrm{i}}\right)=\mathrm{Id} \quad \text { and } \quad \frac{\partial \Phi}{\partial a}=-\mathcal{F}
$$

Inverting the time we get

$$
d F_{1}=\mathrm{Id}-\mathcal{F}\left(\ell_{4}^{\mathrm{i}}\right) \otimes \frac{D \ell_{4}^{\mathrm{i}}}{D \mathcal{V}^{\mathrm{i}}} .
$$

$$
d F_{3}=\left(\operatorname{Id}-\mathcal{F}\left(\ell_{4}^{\mathrm{f}}\right) \otimes \frac{D \ell_{4}^{\mathrm{f}}}{D \mathcal{V}^{\mathrm{f}}}\right)^{-1}
$$

Finally,

$$
d F_{2}=\frac{D \mathcal{V}\left(\ell_{*}^{\mathrm{f}}\right)}{D \mathcal{V}\left(\ell_{*}^{\mathrm{i}}\right)}
$$

is just the fundamental solution of the variational equation between the times $\ell_{*}^{\mathrm{i}}$ and $\ell_{*}^{\mathrm{f}}$. Thus, we get

$$
\mathcal{D}=\left(\operatorname{Id}-\mathcal{F}\left(\ell_{4}^{\mathrm{f}}\right) \otimes \frac{D \ell_{4}^{\mathrm{f}}}{D \mathcal{V}^{\mathrm{f}}}\right)^{-1} \frac{D \mathcal{V}\left(\ell_{4}^{\mathrm{f}}\right)}{D \mathcal{V}\left(\ell_{4}^{\mathrm{i}}\right)}\left(\operatorname{Id}-\mathcal{F}\left(\ell_{4}^{\mathrm{i}}\right) \otimes \frac{D \ell_{4}^{\mathrm{i}}}{D \mathcal{V}^{\mathrm{i}}}\right)
$$

To invert

we need

$$
\operatorname{Id}-\mathcal{F}\left(\ell_{4}^{\mathrm{f}}\right) \otimes \frac{D \ell_{4}^{\mathrm{f}}}{D \mathcal{V}^{\mathrm{f}}}
$$

$$
\left|\frac{D \ell_{4}^{\mathrm{f}}}{D \mathcal{V}^{\mathrm{f}}} \cdot \mathcal{F}\left(\ell_{4}^{\mathrm{f}}\right)\right|<1
$$

Suppose this inequality is satisfied. Indeed for all the cases that we encounter in this paper, the inner product is at most $O(\mu)$. We use

$$
\left(\mathrm{Id}-\mathcal{F}\left(\ell_{4}^{\mathrm{f}}\right) \otimes \frac{D \ell_{4}^{\mathrm{f}}}{D \mathcal{V}^{\mathrm{f}}}\right)^{-1}=\mathrm{Id}+\frac{1}{1-\frac{D \ell_{4}^{\mathrm{f}}}{D \mathcal{V}^{\mathrm{f}}} \cdot \mathcal{F}\left(\ell_{4}^{\mathrm{f}}\right)} \mathcal{F}\left(\ell_{4}^{\mathrm{f}}\right) \otimes \frac{D \ell_{4}^{\mathrm{f}}}{D \mathcal{V}^{\mathrm{f}}} .
$$

Definition 7.1. We call the two terms $d F_{1}$ and $d F_{3}$ the boundary contributions. 


\subsection{Estimates of the variational equation}

Recall $\mathrm{u}\left(\ell_{4}\right), \mathrm{v}\left(\ell_{4}\right)$ defined in Lemmas 6.3 and 6.5 respectively and define further

$$
\left\{\begin{array} { l } 
{ \mathrm { u } ^ { R } = \frac { 1 } { \chi ^ { 3 } } + \frac { \mu } { | \ell _ { 4 } | ^ { 3 } + 1 } , } \\
{ \mathrm { u } ^ { L } = \frac { 1 } { \chi ^ { 3 } } , }
\end{array} \quad \left\{\begin{array} { l } 
{ \mathrm { v } ^ { R } = \frac { 1 } { \chi ^ { 2 } } + \frac { \mu } { | \ell _ { 4 } | ^ { 3 } + 1 } , } \\
{ \mathrm { v } ^ { L } = \frac { 1 } { \chi ^ { 2 } } , }
\end{array} \quad \left\{\begin{array}{l}
\mathrm{w}^{R}=\frac{1}{\chi}+\frac{\mu}{\left|\ell_{4}\right|^{3}+1}, \\
\mathrm{w}^{L}=\frac{1}{\chi} .
\end{array}\right.\right.\right.
$$

When the superscripts $R$ and $L$ are omitted, we use $u, v$ and $w$ to represent either the right or left case depending on the context.

The lemmas in this and the next two sections will be under the standard assumption AG. For the same reason as Corollary 6.10, we can use all the conclusions of Lemma 6.5, since its assumptions are implied by $\mathbf{A G}$ due to Lemmas 6.7 and 6.6.

We start with the following auxiliary estimate. We use the notation $\partial$ to denote the partial derivative with respect to the twelve Delaunay variables, and by $\nabla \mathcal{V}$ the covariant derivative with respect to the ten variables $\mathcal{V}$, where $L_{4}$ is solved for on the zero-energy level. In particular, we have

$$
\nabla \mathcal{V}=\frac{\partial}{\partial \mathcal{V}}+\frac{\partial}{\partial L_{4}} \otimes \nabla_{\mathcal{V}} L_{4}
$$

Note that, for the covariant derivative, we think of $\ell_{4}$ as time, and do not take derivatives with respect to it.

Lemma 7.2. Assume AG. Then, we have the following estimates:

(a)

$$
\nabla_{\mathcal{V}} L_{4}=\left(1,0_{1 \times 9}\right)+O\left(\mu, \mathrm{u}, \mathrm{u}, \mathrm{u} ; \frac{1}{\mu \chi^{2}}, \frac{1}{\chi^{3}}, \mu, \frac{\mu}{\chi} ; \mathrm{v}, \mathrm{v}\right)
$$

(b)

$$
\nabla_{\mathcal{V}}\left(\frac{d t}{d \ell_{4}}\right)=-3 L_{3}^{2}\left(1,0_{1 \times 9}\right)+O\left(\mu, \mathrm{u}, \mathrm{u}, \mathrm{u} ; \frac{1}{\mu \chi^{2}}, \frac{1}{\chi^{3}}, \mu, \frac{\mu}{\chi} ; \mathrm{w}, \mathrm{w}\right)
$$

(c) Corollary 6.10 can be simplified as

$$
\mathcal{F}=\left(0,-1,0_{1 \times 8}\right)+O\left(\mathrm{u}, \mu, \mathrm{u}, \mathrm{u} ; \mu, \frac{\mu}{\chi}, \frac{1}{\mu \chi^{2}}, \frac{1}{\chi^{3}} ; \mathrm{v}, \mathrm{v}\right) .
$$

Proof. We use the same argument as in the proof of Lemma 6.5 to estimate the angles among the vectors $x_{4}, x_{1}, \partial x_{4} / \partial G_{4}$, etc. Part (a) follows directly from equation (6.1). That is, for each of the ten variables $\mathcal{V}$, we apply $\nabla_{\mathcal{V}}$ to both sides of equation (6.1), and then apply the above formula for $\nabla_{\mathcal{V}}$ to $\nabla_{\mathcal{V}}\left(E_{1}+U\right)$. This results in a term with $\nabla_{\mathcal{V}} L_{4}$ on the right side of the equation, so we bring that term to the left side and solve for $\nabla_{\mathcal{V}} L_{4}$. Part (b) follows from equations (6.2) and (6.1). 
Part (a) and (b) differ only in their $G_{4}$ and $g_{4}$ components. The estimates of the $G_{4}$ and $g_{4}$ components in part (a) is the same as that of Lemma 6.5. However, for part (b), we have a $\partial U / \partial L_{4}$ term in (6.2). As a result, we do not have the almost orthogonality of $\partial x_{4} / \partial G_{4}$ with $x_{1}$, as we did in the proof of Lemma 6.5.

We also need to figure out the order of magnitude of each entry of the right-hand side of the variational equation.

Lemma 7.3. Assume AG. Then,

(a)

$\nabla \nu \mathcal{F} \lesssim\left[\begin{array}{cccc|cccc|cc}\mathrm{u} & \mathrm{u} & \mathrm{u} & \mathrm{u} & \mathrm{u} / \chi & \mathrm{u} / \chi & \mu \mathrm{u} & \mathrm{u} / \chi & \mathrm{u} & \mathrm{u} \\ \mu & \mathrm{u} & \mathrm{u} & \mathrm{u} & 1 / \mu \chi^{2} & 1 / \chi^{3} & \mu & \mu / \chi & \mathrm{w} & \mathrm{w} \\ \mathrm{u} & \mathrm{u} & \mathrm{u} & \mathrm{u} & \mathrm{u} / \chi & \mathrm{u} / \chi & \mu \mathrm{u} & \mathrm{u} / \chi & \mathrm{u} & \mathrm{u} \\ \mathrm{u} & \mathrm{u} & \mathrm{u} & \mathrm{u} & \mathrm{u} / \chi & \mathrm{u} / \chi & \mu \mathrm{u} & \mathrm{u} / \chi & \mathrm{u} & \mathrm{u} \\ \hline \mu & \mathrm{u} \mu & \mathrm{u} \mu & \mathrm{u} \mu & 1 / \chi^{2} & \mu / \chi^{3} & \mu & \mu^{2} / \chi & \mu \mathrm{w} & \mu \mathrm{w} \\ \mu / \chi & \mathrm{u} \mu / \chi & \mathrm{u} \mu / \chi & \mathrm{u} \mu / \chi & 1 / \chi^{3} & \mu / \chi^{4} & \mu^{2} / \chi & \mu & \mu \mathrm{w} / \chi & \mu \mathrm{w} / \chi \\ 1 / \mu \chi^{2} & \mathrm{u} / \chi & \mathrm{u} / \chi & \mathrm{u} / \chi & 1 / \mu \chi^{3} & 1 / \chi^{4} & 1 / \chi^{2} & 1 / \chi^{3} & 1 / \chi^{2} & 1 / \chi^{2} \\ 1 / \chi^{2} & \mathrm{u} \chi & \mathrm{u} / \chi & \mathrm{u} / \chi & 1 / \mu \chi^{4} & 1 / \mu \chi^{3} & \mu / \chi^{2} & \mu / \chi^{3} & 1 / \chi^{2} & 1 / \chi^{2} \\ \hline \mathrm{w} & \mathrm{u} & \mathrm{u} & \mathrm{u} & \mathrm{w} / \chi & \mathrm{w} / \chi & \mu \mathrm{w} & \mathrm{w} / \chi & \mathrm{w} & \mathrm{w} \\ \mathrm{w} & \mathrm{u} & \mathrm{u} & \mathrm{u} & \mathrm{w} / \chi & \mathrm{w} / \chi & \mu \mathrm{w} & \mathrm{w} / \chi & \mathrm{w} & \mathrm{w}\end{array}\right]$

(b) In addition, setting $\xi=\left|x_{4}\right| / \chi$, we have in the right case

$$
\nabla_{\mathcal{V}_{4}^{R}} \mathcal{F}_{4}^{R}=\frac{1}{\chi} \frac{\xi}{(1-\xi)^{3}}\left[\begin{array}{cc}
-\frac{L^{4} \operatorname{sign}\left(v_{4, \|}\right)}{G^{2}+L^{2}} & L^{3} \\
\frac{-L^{5}}{\left(G^{2}+L^{2}\right)^{2}} & \frac{L^{4} \operatorname{sign}\left(v_{4, \|}\right)}{G^{2}+L^{2}}
\end{array}\right]+O\left(\frac{\mu}{\chi}+\frac{\mu}{\left|Q_{4}\right|^{2}}\right),
$$

and in the left case

$$
\nabla_{\mathcal{V}_{4}^{L}} \mathcal{F}_{4}^{L}=-\frac{1}{\chi} \frac{\xi}{(1-\xi)^{3}}\left[\begin{array}{cc}
L^{2} \operatorname{sign}\left(v_{4, \|}\right) & L^{3} \\
-L & -L^{2} \operatorname{sign}\left(v_{4, \|}\right)
\end{array}\right]+O\left(\frac{\mu}{\chi}\right) .
$$

Proof. The proof is organized as follows. We start with a formal computation which gives the formula for estimating each block of the matrices. Next, we work on the estimate of each block. In the left case, a near collision may occur. Since we treat $\ell_{4}$ as the new time, we do not take derivatives with respect to $\ell_{4}$ when deriving the variational equation, so in the Hamiltonian equations, as well as the variational equation, we need only $C^{0}$ dependence on $\ell_{4}$, which is always true (see (A.3) and (A.4)) even when we have a collision between $Q_{1}$ and $Q_{4}$. In the estimate of the rows related to $G_{4}$ and $g_{4}$ in the left case, we will need Lemma A.1. 


\section{- A formal computation}

Using our notation, the two matrices are $d \mathcal{F} / d \mathcal{V}$. They are the coefficient matrices of the variational equations

$$
\frac{d}{d \ell_{4}} \delta \mathcal{V}=\frac{d \mathcal{F}}{d \mathcal{V}} \delta \mathcal{V}
$$

We split each of the two matrices into nine blocks corresponding to $\partial \mathcal{F}_{i} / \partial \mathcal{V}_{j}$, where $i, j=3,1,4$.

We have $d \mathcal{F} / d \mathcal{V}=\nabla_{\mathcal{V}} \mathcal{F}$. Notice that

$$
\mathcal{F}=\frac{d t}{d \ell_{4}} J \frac{\partial H}{\partial \mathcal{V}}
$$

where $J$ is the standard symplectic matrix. Then we get the formal expression to calculate the two matrices

$$
\nabla_{\mathcal{V}_{j}} \mathcal{F}_{i}=J_{i} \frac{\partial H}{\partial \mathcal{V}_{i}} \otimes\left(\nabla_{\mathcal{V}_{j}} \frac{d t}{d \ell_{4}}\right)+\frac{d t}{d \ell_{4}}\left(\nabla_{\mathcal{V}_{j}} J_{i} \frac{\partial H}{\partial \mathcal{V}_{i}}\right)
$$

Note that $\nabla_{\mathcal{V}} d t / d \ell_{4}$ is done in Lemma 7.2, and $J_{i} \partial H / \partial \mathcal{V}_{i}$ is done in Corollary 6.10, the term $d t / d \ell_{4}=O(1)$ and the new term we need to consider is $\nabla_{\mathcal{V}_{j}} J_{i} \partial H / \partial \mathcal{V}_{i}$. For $i, j=3,1,4$, we have

$$
\begin{aligned}
\nabla_{\mathcal{V}_{j}} J_{i} \frac{\partial H}{\partial \mathcal{V}_{i}}= & \frac{\partial}{\partial \mathcal{V}_{j}} J_{i} \frac{\partial H}{\partial \mathcal{V}_{i}}+\frac{\partial}{\partial L_{4}} J_{i} \frac{\partial H}{\partial \mathcal{V}_{i}} \otimes \nabla_{\mathcal{V}_{j}} L_{4} \\
= & \frac{\partial \mathcal{X}_{j}}{\partial \mathcal{V}_{j}} \frac{\partial}{\partial \mathcal{X}_{j}}\left(J_{i} \frac{\partial H}{\partial \mathcal{X}_{i}} \frac{\partial \mathcal{X}_{i}}{\partial \mathcal{V}_{i}}\right)+\frac{\partial}{\partial L_{4}}\left(J_{i} \frac{\partial H}{\partial \mathcal{X}_{i}} \frac{\partial \mathcal{X}_{i}}{\partial \mathcal{V}_{i}}\right) \otimes \nabla \mathcal{V}_{j} L_{4} \\
= & \frac{\partial \mathcal{X}_{j}}{\partial \mathcal{V}_{j}} J_{i} \frac{\partial^{2} H}{\partial \mathcal{X}_{j} \partial \mathcal{X}_{i}} \frac{\partial \mathcal{X}_{i}}{\partial \mathcal{V}_{i}}+J_{i} \frac{\partial H}{\partial \mathcal{X}_{i}} \frac{\partial^{2} \mathcal{X}_{i}}{\partial \mathcal{V}_{j} \partial \mathcal{V}_{i}} \\
& +\left(\frac{\partial \mathcal{X}_{4}}{\partial L_{4}} J_{i} \frac{\partial^{2} H}{\partial \mathcal{X}_{4} \partial \mathcal{X}_{i}} \frac{\partial \mathcal{X}_{i}}{\partial \mathcal{V}_{i}}+J_{i} \frac{\partial H}{\partial \mathcal{X}_{i}} \frac{\partial^{2} \mathcal{X}_{i}}{\partial L_{4} \partial \mathcal{V}_{i}}\right) \otimes \nabla_{\mathcal{V}_{j}} L_{4}
\end{aligned}
$$

where $J_{i}$ is the standard symplectic matrix in the $i$ component. We know by Lemma 6.6 that

$$
\frac{\partial \mathcal{X}_{3}}{\partial \mathcal{V}_{3}}=O(1), \quad \frac{\partial \mathcal{X}_{1}}{\partial \mathcal{V}_{1}}=\operatorname{Id}_{4} \quad \text { and } \quad \frac{\partial \mathcal{X}_{4}}{\partial L_{4}}, \frac{\partial \mathcal{X}_{4}}{\partial \mathcal{V}_{4}}=O\left(\ell_{4}\right)
$$

according to Lemma A.3. Moreover, $\partial H / \partial \mathcal{X}_{j}$ and $\partial^{2} H / \partial \mathcal{X}_{i} \partial \mathcal{X}_{j}$ are done in Lemma 6.3, $\partial^{2} \mathcal{X}_{j} / \partial \mathcal{V}_{i} \partial \mathcal{V}_{j}$ and $\partial^{2} \mathcal{X}_{j} / \partial L_{4} \partial \mathcal{V}_{j}$ are done in Lemma A.6 and, finally, $\partial L_{4} / \partial \mathcal{V}_{i}$ is done in Lemma 7.2. Now every term in (7.3) and (7.4) is already estimated. What we need to do below is to find the leading term for each matrix entry among all the terms above.

In the following we analyze the two matrices blockwise. We will handle the left and right cases simultaneously. 


\section{- $\nabla_{\mathcal{V}_{3}} \mathcal{F}_{3}$}

For this block, the $(2,1)$ entry is special. All the remaining entries are done together.

Using the Hamiltonian equations $(6.3)$, we see that the $(2,1)$ entry is

$$
\begin{aligned}
& \nabla_{L_{3}}\left(\frac{d t}{d \ell_{4}}\left(\frac{m_{3} k_{3}^{2}}{L_{3}^{3}}+\frac{\partial U}{\partial L_{3}}\right)\right) \\
& \quad=-\nabla_{L_{3}}\left(m_{3} k_{3}^{2}\left(1+L_{3}^{2}\left(\frac{v_{1}^{2}}{2 m_{1}}-\frac{k_{1}}{\left|x_{1}\right|}\right)+3 U L_{3}^{2}\right)-L_{3}^{6} \frac{\partial U}{\partial L_{4}}+L_{3}^{3} \frac{\partial U}{\partial L_{3}}+\text { h.o.t. }\right) .
\end{aligned}
$$

The leading term is

$$
\nabla_{L_{3}} L_{3}^{2} \cdot\left(\frac{v_{1}^{2}}{2 m_{1}}-\frac{k_{1}}{\left|x_{1}\right|}\right)=O(\mu)
$$

since

$$
m_{1}=O\left(\frac{1}{\mu}\right), \quad L_{3}=O(1), \quad v_{1}=O(1) \quad \text { and } \quad\left|x_{1}\right|=O(\chi) .
$$

All the other subleading terms involve derivatives of $U$, which are at most w coming from $\nabla_{L_{3}} \partial U / \partial L_{4}$. This completes the estimate of the $(2,1)$ entry. The other three entries in the second row are estimated by u mainly contributed by $\partial U / \partial x_{3}$ (Lemma 6.3).

For the first, third and fourth rows, we use formula (7.3). The first summand in (7.3) contributes $(\mathrm{u}, \mathrm{u}, \mathrm{u}) \otimes(\mathrm{u}, 1, \mathrm{u}, \mathrm{u})$ to the three rows. The second summand is given by (7.4). The first and second terms after the third equal sign in (7.4) have the same estimates,

$$
\frac{\partial U}{\partial x_{3}}, \frac{\partial^{2} U}{\partial x_{3}^{2}} \lesssim \mathbf{u}
$$

as we get in Lemma 6.3, since

$$
\frac{\partial \mathcal{X}_{3}}{\partial \mathcal{V}_{3}}, \frac{\partial^{2} \mathcal{X}_{3}}{\partial \mathcal{V}_{3}^{2}}=O(1) \quad \text { and } \quad \nabla_{\mathcal{V}_{3}} L_{4}=O(1)
$$

using Lemma 7.2. The summand

$$
J_{3} \frac{\partial H}{\partial \mathcal{X}_{3}} \frac{\partial^{2} \mathcal{X}_{3}}{\partial L_{4} \partial \mathcal{V}_{3}} \otimes \nabla \mathcal{V}_{3} L_{4}=0
$$

since $\partial^{2} \mathcal{X}_{3} / \partial L_{4} \partial \mathcal{V}_{3}=0$. The third term in (7.4) is estimated as $\mu /\left(\left|\ell_{4}\right|^{3}+1\right)$ in the right case and $\mu / \chi^{3}$ in the left case using the estimate $\partial^{2} U / \partial x_{3} \partial x_{4}$ in Lemma 6.3 and the fact that $\partial \mathcal{X}_{4} / \partial L_{4}=O\left(\ell_{4}\right)$. So, in summary, all the entries in the three rows are bounded by u. 
- $\nabla_{\mathcal{V}_{1}} \mathcal{F}_{3}$

The first summand in (7.3) gives

$$
(\mathrm{u}, 1, \mathrm{u}, \mathrm{u}) \otimes\left(\frac{1}{\mu \chi^{2}}, \frac{1}{\chi^{3}}, \mu, \frac{\mu}{\chi}\right) .
$$

The second summand in (7.3) is now reduced to

$$
J_{3} \frac{\partial^{2} U}{\partial x_{1} \partial x_{3}} \frac{\partial x_{3}}{\partial \mathcal{V}_{3}}+\frac{\partial x_{4}}{\partial L_{4}} J_{3} \frac{\partial^{2} U}{\partial x_{4} \partial x_{3}} \frac{\partial x_{3}}{\partial \mathcal{V}_{3}} \otimes \nabla_{\mathcal{V}_{1}} L_{4}
$$

since

$$
\frac{\partial \mathcal{X}_{1}}{\partial \mathcal{V}_{1}}=\mathrm{Id} \quad \text { and } \quad \frac{\partial^{2} \mathcal{X}_{3}}{\partial \mathcal{V}_{1} \partial \mathcal{V}_{3}}=\frac{\partial^{2} \mathcal{X}_{3}}{\partial L_{4} \partial \mathcal{V}_{3}}=0
$$

The two terms are estimated using

$$
\frac{\partial^{2} U}{\partial x_{1} \partial x_{3}} \lesssim \frac{1}{\chi^{4}}
$$

given by Lemma 6.3,

$$
\nabla_{\mathcal{V}_{1}} L_{4} \lesssim\left(\frac{1}{\mu \chi^{2}}, \frac{1}{\chi^{3}}, \mu, \frac{\mu}{\chi}\right)
$$

by Lemma 7.2 and

$$
\left(\frac{\partial x_{4}}{\partial L_{4}} J_{j} \frac{\partial^{2} U}{\partial x_{4} \partial x_{3}} \frac{\partial x_{3}}{\partial \mathcal{V}_{3}}\right) \lesssim \mathbf{u},
$$

since we have $\partial \mathcal{X}_{4} / \partial L_{4}=O\left(\ell_{4}\right)$, and

$$
\frac{\partial^{2} H}{\partial \mathcal{X}_{4} \partial \mathcal{X}_{3}} \lesssim \frac{\mu}{\ell_{4}^{4}+1}
$$

in the right case and

$$
\frac{\partial^{2} H}{\partial \mathcal{X}_{4} \partial \mathcal{X}_{3}} \lesssim \frac{\mu}{\chi^{4}}
$$

in the left case using Lemma 6.3. Therefore, the estimate of the block is given by the max of

$$
(\mathrm{u}, 1, \mathrm{u}, \mathrm{u}) \otimes\left(\frac{1}{\mu \chi^{2}}, \frac{1}{\chi^{3}}, \mu, \frac{\mu}{\chi}\right) \text { and } \frac{1}{\chi^{4}} .
$$

\section{- $\nabla \mathcal{V}_{4} \mathcal{F}_{3}$}

The second row is handled in a similar manner to the $\nabla_{\mathcal{V}_{3}} \mathcal{F}_{3}$ block. Thus,

$$
\begin{array}{r}
\nabla_{\mathcal{V}_{4}}\left(\frac{d t}{d \ell_{4}}\left(\frac{m_{3} k_{3}^{2}}{L_{3}^{3}}+\frac{\partial U}{\partial L_{3}}\right)\right)=-\nabla \mathcal{V}_{4}\left(m_{3} k_{3}^{2}\left(1+3 L_{3}^{2}\left(\frac{v_{1}^{2}}{2 m_{1}}-\frac{k_{1}}{\left|x_{1}\right|}\right)+3 U L_{3}^{2}\right)\right. \\
\left.-L_{3}^{6} \frac{\partial U}{\partial L_{4}}+L_{3}^{3} \frac{\partial U}{\partial L_{3}}+\text { h.o.t. }\right)
\end{array}
$$




$$
=-\nabla \mathcal{V}_{4}\left(3 U L_{3}^{2}-L_{3}^{6} \frac{\partial U}{\partial L_{4}}+L_{3}^{3} \frac{\partial U}{\partial L_{3}}+\text { h.o.t. }\right)
$$

The leading term is given by

$$
\nabla_{\mathcal{V}_{4}} \frac{\partial U}{\partial L_{4}}=\left(\frac{\partial \mathcal{X}_{4}}{\partial L_{4}} J_{4} \frac{\partial^{2} U}{\partial x_{4}^{2}} \nabla_{\mathcal{V}_{4}} x_{4}\right) \lesssim \mathrm{w}
$$

using Lemma 6.3.

Next, the first summand in $(7.3)$ gives the estimate $(u, u, u) \otimes(w, w)$ for the first, third and fourth row. This is smaller than what we have stated in the lemma. It remains to consider (7.4), which is reduced to

$$
\frac{\partial x_{4}}{\partial \mathcal{V}_{4}} J_{3} \frac{\partial^{2} U}{\partial x_{4} \partial x_{3}} \frac{\partial x_{3}}{\partial \mathcal{V}_{3}}+\frac{\partial x_{4}}{\partial L_{4}} J_{3} \frac{\partial^{2} U}{\partial x_{4} \partial x_{3}} \frac{\partial x_{3}}{\partial \mathcal{V}_{3}} \otimes \nabla_{\mathcal{V}_{4}} L_{4}
$$

since

$$
\frac{\partial x_{3}}{\partial \mathcal{V}_{4}}=\frac{\partial x_{3}}{\partial L_{4}}=0
$$

The first summand has the estimate $\mu /\left(\ell_{4}^{3}+1\right)$ in the right case and $\mu / \chi^{3}$ in the left case, since we have the estimate $\partial x_{4} / \partial \mathcal{V}_{4}=O\left(\ell_{4}\right)$ and

$$
\frac{\partial^{2} H}{\partial \mathcal{X}_{4} \partial \mathcal{X}_{3}} \lesssim \frac{\mu}{\ell_{4}^{4}+1}
$$

in the right case and

$$
\frac{\partial^{2} H}{\partial \mathcal{X}_{4} \partial \mathcal{X}_{3}} \lesssim \frac{\mu}{\chi^{4}}
$$

in the left case in Lemma 6.3. So, we use $\mathrm{u}$ to bound this first summand as stated in the lemma. For the second summand in (7.4), we estimate $\nabla_{\mathcal{V}_{4}} L_{4}$ as $(\mathrm{v}, \mathrm{v})$ using Lemma 7.2, and

$$
\frac{\partial x_{4}}{\partial L_{4}} J_{4} \frac{\partial^{2} U}{\partial x_{4} \partial x_{3}} \frac{\partial x_{3}}{\partial \mathcal{V}_{3}}
$$

has the same estimate as the first summand. So, the second summand is much smaller than the first summand.

For the next three blocks $\nabla_{\mathcal{V}_{j}} \mathcal{F}_{1}, j=3,1,4$, using $\partial \mathcal{X}_{1} / \partial \mathcal{V}_{1}=\mathrm{Id},(7.4)$ is reduced to

$$
\frac{\partial \mathcal{X}_{j}}{\partial \mathcal{V}_{j}} J_{1} \frac{\partial^{2} H}{\partial \mathcal{X}_{j} \partial \mathcal{X}_{1}}+\frac{\partial x_{4}}{\partial L_{4}} J_{1} \frac{\partial^{2} H}{\partial x_{4} \partial \mathcal{X}_{1}} \otimes \nabla_{\mathcal{V}_{j}} L_{4}
$$


- $\nabla_{\mathcal{V}_{3}} \mathcal{F}_{1}$

The first summand in (7.3) gives

$$
\left(\mu, \frac{\mu}{\chi}, \frac{1}{\mu \chi^{2}}, \frac{1}{\chi^{3}}\right) \otimes(1, \mathrm{u}, \mathrm{u}, \mathrm{u})
$$

The first summand in (7.5) has the same estimate as

$$
\frac{\partial^{2} H}{\partial x_{3} \partial x_{1}}=\frac{\partial^{2} U}{\partial x_{3} \partial x_{1}} \lesssim \frac{1}{\chi^{4}}, \frac{\partial^{2} H}{\partial \mathcal{X}_{3} \partial v_{1}}=0
$$

using Lemma 6.3, whose contribution to the current block is

$$
\left[\begin{array}{c}
0_{2 \times 4} \\
\left(1 / \chi^{4}\right)_{2 \times 4}
\end{array}\right]
$$

Next, we consider the second summand in (7.5). We have $\nabla_{\mathcal{V}_{3}} L_{4} \lesssim(1, \mathrm{u}, \mathrm{u}, \mathrm{u})$ using Lemma 7.2. Next, we consider

$$
\frac{\partial x_{4}}{\partial L_{4}} J_{1} \frac{\partial^{2} H}{\partial x_{4} \partial \mathcal{X}_{1}} .
$$

This is a vector of four entries whose first two entries are

$$
\frac{\partial^{2} H}{\partial L_{4} \partial v_{1}}=\frac{\partial v_{1}}{\partial L_{4}}=0
$$

and whose last two entries are bounded by $1 / \chi^{2}$, since we have

$$
\frac{\partial x_{4}}{\partial L_{4}}=O\left(\ell_{4}\right)
$$

and

$$
\frac{\partial^{2} H}{\partial x_{4} \partial x_{1}} \lesssim \frac{1}{\chi^{3}}
$$

using Lemma 6.3. So, we use $(\mu, \mu / \chi) \otimes(1, \mathrm{u}, \mathrm{u}, \mathrm{u})$ as the estimate for the first two rows and use the max of

$$
\left(\frac{1}{\mu \chi^{2}}, \frac{1}{\chi^{3}}\right) \otimes\left(1,(\mathrm{u})_{1 \times 3}\right), \quad\left(\frac{1}{\chi^{4}}\right)_{2 \times 4} \text { and }\left(\frac{1}{\chi^{2}}, \frac{1}{\chi^{2}}\right) \otimes(1, \mathrm{u}, \mathrm{u}, \mathrm{u})
$$

as the estimate for the last two rows. 
- $\nabla_{\mathcal{V}_{1}} \mathcal{F}_{1}$

The first summand in (7.3) gives

$$
\left(\mu, \frac{\mu}{\chi}, \frac{1}{\mu \chi^{2}}, \frac{1}{\chi^{3}}\right) \otimes\left(\frac{1}{\mu \chi^{2}}, \frac{1}{\chi^{3}}, \mu, \frac{\mu}{\chi}\right) .
$$

The first summand in (7.5) is

$$
\left[\begin{array}{cc}
\frac{\partial^{2} H}{\partial x_{1} \partial v_{1}} & \frac{\partial^{2} H}{\partial v_{1} \partial v_{1}} \\
-\frac{\partial^{2} H}{\partial x_{1}^{2}} & -\frac{\partial^{2} H}{\partial x_{1} \partial v_{1}}
\end{array}\right]=\left[\begin{array}{cc}
0 & \frac{1}{m_{1}} \text { Id } \\
\frac{3 k_{1} x_{1} \otimes x_{1}}{\left|x_{1}\right|^{5}}-\frac{k_{1} \text { Id }}{\left|x_{1}\right|^{3}} & 0
\end{array}\right],
$$

where $\partial^{2} H / \partial x_{1}^{2}$ is given by $\partial^{2} / \partial x_{1}^{2} \frac{k_{1}}{\left|x_{1}\right|}$. We compare the two matrices using $x_{1}=O(\chi, \mu)$ to get the first three rows. Finally we consider the second summand in (7.5). We notice that

$$
\nabla_{\mathcal{V}_{1}} L_{4} \lesssim\left(\frac{1}{\mu \chi^{2}}, \frac{1}{\chi^{3}}, \mu, \frac{\mu}{\chi}\right)
$$

The term

$$
\left(\frac{\partial x_{4}}{\partial L_{4}} J_{1} \frac{\partial^{2} H}{\partial x_{4} \partial \mathcal{X}_{1}}\right) \lesssim\left(0,0, \frac{1}{\chi^{2}}, \frac{1}{\chi^{2}}\right)
$$

as in the previous paragraph. This gives us the last row.

\section{- $\nabla_{\mathcal{V}_{4}} \mathcal{F}_{1}$}

The first summand in (7.3) gives

$$
\left(\mu, \frac{\mu}{\chi}, \frac{1}{\mu \chi^{2}}, \frac{1}{\chi^{3}}\right) \otimes(\mathrm{w}, \mathrm{w})
$$

using Lemma 7.2 and Corollary 6.10. This gives the first two rows in both matrices. The first two rows of (7.5) are zero because $\partial^{2} H / \partial x_{4} \partial v_{1}=0$. For the last two rows in (7.5), we first have

$$
\left(\frac{\partial x_{4}}{\partial \mathcal{V}_{4}}\right)\left(J_{1} \frac{\partial^{2} U}{\partial x_{4} \partial x_{1}}\right) \lesssim \frac{1}{\chi^{2}} \quad \text { and } \quad \frac{\partial x_{4}}{\partial L_{4}} J_{1} \frac{\partial^{2} U}{\partial x_{4} \partial x_{1}} \lesssim \frac{1}{\chi^{2}}
$$

as in the previous paragraph, so the tensor part is $O\left(\mathrm{v} / \chi^{2}\right)$. Thus, for the last two rows, we use the estimate $1 / \chi^{2}$. 


\section{- $\nabla_{\mathcal{V}_{3}} \mathcal{F}_{4}$}

The first summand in $(7.3)$ gives $(\mathrm{w}, \mathrm{w}) \otimes(1, \mathrm{u}, \mathrm{u}, \mathrm{u})$. The first summand in (7.4) is given by

$$
\frac{\partial x_{3}}{\partial \mathcal{V}_{3}}\left(J_{3} \frac{\partial^{2} U}{\partial x_{4} \partial x_{3}}\right) \frac{\partial x_{4}}{\partial \mathcal{V}_{4}} \lesssim \mathbf{u},
$$

using Lemma 6.3. This gives the second, third and fourth columns of the block.

Next, the second summand in (7.4) vanishes, since $\partial^{2} x_{4} / \partial \mathcal{V}_{3} \partial \mathcal{V}_{4}=0$.

It remains to consider the third summand in (7.4). We have $\nabla_{\mathcal{V}_{3}} L_{4} \lesssim\left(1,(\mathbf{u})_{1 \times 3}\right)$. Next,

$$
J_{4} \frac{\partial U}{\partial x_{4}} \frac{\partial x_{4}}{\partial L_{4} \partial \mathcal{V}_{4}} \lesssim \ell_{4}\left(\frac{1}{\chi^{2}}+\frac{\mu}{\ell_{4}^{4}+1}\right) \lesssim \mathrm{w},
$$

using Lemmas 6.3 and A.6. Next,

$$
\frac{\partial x_{4}}{\partial L_{4}}\left(J_{4} \frac{\partial^{2} U}{\partial x_{4} \partial x_{4}}\right) \frac{\partial x_{4}}{\partial \mathcal{V}_{4}} \lesssim \mathrm{w} .
$$

So, the third summand in $(7.4)$ has the estimates $(w)_{1 \times 2} \otimes\left(1,(u)_{1 \times 3}\right)$ and contributes to the first column of the block along with the the first summand of (7.3).

\section{- $\nabla \mathcal{V}_{1} \mathcal{F}_{4}$}

The first summand in (7.3) gives

$$
(\mathrm{w}, \mathrm{w}) \otimes\left(\frac{1}{\mu \chi^{2}}, \frac{1}{\chi^{3}}, \mu, \frac{\mu}{\chi}\right) .
$$

The first summand in (7.4) is given by

$$
\left(J_{4} \frac{\partial^{2} U}{\partial x_{4} \partial x_{1}}\right) \frac{\partial \mathcal{X}_{4}}{\partial \mathcal{V}_{4}} \lesssim \frac{1}{\chi^{2}},
$$

using Lemma 6.3. The second summand in (7.4) vanishes, since $\partial^{2} x_{4} / \partial \mathcal{V}_{1} \partial \mathcal{V}_{4}=0$. Finally, we consider the third summand in (7.4). We have

$$
\nabla_{\mathcal{V}_{1}} L_{4} \lesssim\left(\frac{1}{\mu \chi^{2}}, \frac{1}{\chi^{3}}, \mu, \frac{\mu}{\chi}\right)
$$

The estimate of

$$
\frac{\partial x_{4}}{\partial L_{4}}\left(J_{4} \frac{\partial^{2} U}{\partial x_{4} \partial x_{4}}\right) \frac{\partial x_{4}}{\partial \mathcal{V}_{4}}+J_{4} \frac{\partial U}{\partial x_{4}} \frac{\partial x_{4}}{\partial L_{4} \partial \mathcal{V}_{4}} \lesssim \mathrm{w}
$$

was done in the previous block.

So the estimate of this block is to take the larger between

$$
\left(\frac{1}{\chi^{2}}\right)_{2 \times 4} \text { and }(\mathrm{w}, \mathrm{w}) \otimes\left(\frac{1}{\mu \chi^{2}}, \frac{1}{\chi^{3}}, \mu, \frac{\mu}{\chi}\right) \text {. }
$$




\section{- $\nabla_{\mathcal{V}_{4}} \mathcal{F}_{4}$}

The leading contribution is given by the first and second summands in (7.4),

$$
\frac{\partial x_{4}}{\partial \mathcal{V}_{4}} J_{4} \frac{\partial^{2} U}{\partial x_{4} \partial x_{4}} \frac{\partial x_{4}}{\partial \mathcal{V}_{4}}+J_{4} \frac{\partial U}{\partial x_{4}} \frac{\partial^{2} x_{4}}{\partial \mathcal{V}_{4} \partial \mathcal{V}_{4}} \lesssim \mathrm{w},
$$

using Lemma 6.3. We next show the other summands are small. The first summand in (7.3) gives $(\mathrm{w}, \mathrm{w}) \otimes(\mathrm{w}, \mathrm{w})$. Next, we consider the second summand in (7.4). We have $\nabla \mathcal{V}_{4} L_{4} \lesssim(\mathrm{v}, \mathrm{v})$. Next,

$$
\frac{\partial x_{4}}{\partial L_{4}}\left(J_{4} \frac{\partial^{2} U}{\partial x_{4} \partial x_{4}}\right) \frac{\partial x_{4}}{\partial \mathcal{V}_{4}}+\left(J_{4} \frac{\partial U}{\partial x_{4}}\right) \frac{\partial^{2} x_{4}}{\partial L_{4} \partial \mathcal{V}_{4}} \lesssim \mathrm{w}
$$

is estimated as before. Therefore, the first and third summands in (7.4) are much smaller than w.

\section{Part (a) is complete now. Finally, we show part (b)}

According to the last bullet point, the leading terms in $\nabla_{\mathcal{V}_{4}} \mathcal{F}_{4}$ come from

$$
\frac{d t}{d \ell_{4}}\left(\frac{\partial \mathcal{X}_{4}}{\partial \mathcal{V}_{4}}\left(J_{j} \frac{\partial^{2} H}{\partial \mathcal{X}_{4} \partial \mathcal{X}_{4}}\right) \frac{\partial \mathcal{X}_{4}}{\partial \mathcal{V}_{4}}+J_{4} \frac{\partial H}{\partial \mathcal{X}_{4}} \frac{\partial^{2} \mathcal{X}_{4}}{\partial \mathcal{V}_{4} \partial \mathcal{V}_{4}}\right)=L_{4}^{3}\left(\frac{\partial}{\partial G_{4}}, \frac{\partial}{\partial g_{4}}\right)\left[\begin{array}{c}
\frac{\partial x_{4}}{\partial g_{4}} \cdot \frac{\partial U}{\partial x_{4}} \\
-\frac{\partial x_{4}}{\partial G_{4}} \cdot \frac{\partial U}{\partial x_{4}}
\end{array}\right] .
$$

Let us now look at $U^{R}$ in (4.3). Only those terms in $U^{R}$ containing both $x_{4}$ and $x_{1}$ can be as large as $O(1 / \chi)$, according to Lemma 6.3. So, we only need to consider the following three terms in $U^{R}$ :

$$
-\left(\frac{1}{\mu\left|x_{1}+\frac{\mu}{1+2 \mu} x_{4}+\frac{\mu}{1+\mu} x_{3}\right|}+\frac{1}{\left|x_{1}+\frac{\mu}{1+2 \mu} x_{4}-\frac{1}{1+\mu} x_{3}\right|}+\frac{1}{\left|x_{1}-\frac{1+\mu}{1+2 \mu} x_{4}\right|}\right) .
$$

When we take two derivatives with respect to $x_{4}$, a $\mu^{2}$ factor will multiply the first two terms, so that the first two terms would be $O(\mu)$, compared to the third term. So, the leading contribution to $\partial^{2} U^{R} / \partial x_{4}^{2}$ is given by

$$
\frac{\partial^{2}}{\partial x_{4}^{2}} \frac{-1}{\left|x_{1}-\frac{1+\mu}{1+2 \mu} x_{4}\right|} .
$$


The same analysis for $U^{L}$ in (4.6) shows the leading contribution to $\partial^{2} U^{L} / \partial x_{4}^{2}$ is given by

$$
\frac{\partial^{2}}{\partial x_{4}^{2}} \frac{-1}{\left|x_{1}+\frac{\mu}{1+\mu} x_{3}+\frac{1+\mu}{1+2 \mu} x_{4}\right|} .
$$

Consider the $(9,9)$ entry. The main contribution to this entry comes from

$$
L_{4}^{3} \frac{\partial}{\partial G_{4}}\left(\frac{\partial x_{4}}{\partial g_{4}} \cdot \frac{\partial U}{\partial x_{4}}\right)=(1+O(\mu)) L_{4}^{3} \frac{\partial}{\partial G_{4}}\left(\frac{\left(\partial x_{4} / \partial g_{4}\right) \cdot\left(x_{4}-\left(1+O(\mu) x_{1}\right)\right)}{\left|x_{4}-\left(1+O(\mu) x_{1}\right)\right|^{3}}\right) .
$$

The numerator on the right-hand side equals

$$
(1+O(\mu)) L_{4}^{3} \frac{\partial}{\partial G_{4}}\left(\frac{\partial x_{4}}{\partial g_{4}} \cdot x_{4}\right)-(1+O(\mu)) L_{4}^{3} \frac{\partial^{2} x_{4}}{\partial G_{4} \partial g_{4}} \cdot x_{1} .
$$

The first term is $O(\chi)$ due to Lemma A.3 (c), so the main contribution comes from the second term, which is $O\left(\chi^{2}\right)$ using Lemma A.6. We use the same argument for the other entries to get

$$
\nabla_{\mathcal{V}_{4}^{R}} \mathcal{F}_{4}^{R}=-L_{3}^{3}\left[\begin{array}{cc}
\frac{\partial^{2} x_{4}}{\partial G \partial g} \cdot \frac{x_{1}}{\left|x_{4}-x_{1}\right|^{3}} & \frac{\partial^{2} x_{4}}{\partial g^{2}} \cdot \frac{x_{1}}{\left|x_{4}-x_{1}\right|^{3}} \\
-\frac{\partial^{2} x_{4}}{\partial G^{2}} \cdot \frac{x_{1}}{\left|x_{4}-x_{1}\right|^{3}} & -\frac{\partial^{2} x_{4}}{\partial G \partial g} \cdot \frac{x_{1}}{\left|x_{4}-x_{1}\right|^{3}}
\end{array}\right]+O\left(\frac{\mu}{\chi}+\frac{\mu}{\left|x_{4}\right|^{3}}\right) .
$$

Using Lemma A.6, we see that the $(9,9)$ entry equals

$$
-\frac{L_{4}^{5}}{\sqrt{L_{4}^{2}+G_{4}^{2}}} \frac{\chi \sinh u}{\left|x_{4}-x_{1}\right|^{3}}+O\left(\frac{\mu}{\chi}+\frac{\mu}{\left|x_{4}\right|^{3}}\right) .
$$

Recall that $L_{3}=L_{4}(1+o(1))$ (due to $\left.(6.1)\right)$ and $\sinh u=\operatorname{sign}(u)\left|\ell_{4}\right| L_{4} / \sqrt{L_{4}^{2}+G_{4}^{2}}$ (due to (A.4)). Since Lemma 6.6 implies that $\left|x_{4}\right|=\left|\ell_{4}\right| / L_{4}^{2}(1+o(1))$, we get that the $O(1 / \chi)$-term in $(9,9)$ is asymptotic to

$$
-\frac{L^{4} \operatorname{sign}(u)}{L^{2}+G^{2}} \frac{\chi\left|x_{4}\right|}{\left(\chi-\left|x_{4}\right|\right)^{3}}
$$

Since $u$ and $v_{4, \|}$ have opposite signs, we obtain the asymptotics of the $O(1 / \chi)$-term claimed in part (b) of Lemma 7.3 for the $(9,9)$ entry. The analysis of other entries of $\nabla_{\mathcal{V}_{4}^{R}} \mathcal{F}_{4}^{R}$ is similar.

Next, we consider the left case. The argument is the same except for the following differences. First, the error term in (7.6) is now $O(\mu / \chi)$, since $\mu /\left|x_{4}\right|^{3}$ should be replaced by $1 / \chi^{3}$, as usual. Next, $U^{L}$ is roughly $1 /\left|x_{4}+x_{1}\right|$, up to some $\mu$ error, which differs from $U^{R}$ by a "minus" sign. Then, we have that the asymptotic expression of (7.7) follows directly from Lemma A.6 (c). 


\subsection{Estimates of the solution of the variational equations}

In this section, we give the proof of the estimates of matrices $N_{1}, N_{3}, N_{5}$ and the (I) $)_{44}$, $(\mathrm{III})_{44},(\mathrm{~V})_{44}$ blocks in Proposition 5.2.

From one Poincaré section to the next, it takes time of order $O(\chi)$. The main body of the proof is to show that the fundamental solutions of the variational equations are estimated by three steps of Picard iterations.

\subsubsection{The asymptotics of the $\left(N_{1}\right)_{44},\left(N_{3}\right)_{44},\left(N_{5}\right)_{44}$ blocks in}

\section{Proposition $5.2(\mathrm{~b})$}

The blocks are obtained by integrating the leading terms in the estimates of $\nabla_{\mathcal{V}_{4}} \mathcal{F}_{4}$ in part (b) of Lemma 7.3. After a rescaling of time, the problem is reduced to finding the fundamental solution of a linear ordinary differential equation (ODE) system defined by constant $2 \times 2$ matrices.

\subsubsection{The matrix $N_{3}$ for the piece (III)}

Let us first explain how to get the matrix $N_{3}$. Since the right matrix of Lemma 7.3 has constant entries, which we denote by $K$ temporarily, $N_{3}$ can be estimated by the fundamental solution of the ODE $X^{\prime}=K \cdot X$, that is, by

$$
X(\chi)=e^{K \chi}=\sum_{n=0}^{\infty} \frac{1}{n !}(K \chi)^{n} .
$$

Note that $K$ has positive entries. We claim that in fact

$$
e^{K \chi}-\operatorname{Id}_{10}=O\left(K \chi+\frac{1}{2}(K \chi)^{2}+(K \chi)^{3}\right) .
$$

Indeed, a brute force calculation shows that $(K \chi)^{4} \leqslant C_{3}\left(K \chi+(K \chi)^{2}+(K \chi)^{3}\right)$. This allows us to get inductively that

$$
(K \chi)^{n} \leqslant C_{n}\left(K \chi+(K \chi)^{2}+(K \chi)^{3}\right) \quad \text { where } C_{n}=C_{3}\left(1+C_{3}\right)^{n-4} .
$$

Summing the series for $e^{K \chi}$, we obtain (7.8). All entry except $(6,5)$ and $(7,8)$ appear in $K \chi+(K \chi)^{2}$.

We remark that the computation can be done either by computer or by hand. Note that the 1st, 3rd and 4th rows, the 9th and 10th rows, the 2nd, 3rd and 4th columns and the 9 th and 10th columns are the same, respectively, so we can reduce the size of the matrices from $10 \times 10$ to $7 \times 7$. 


\subsubsection{The matrices $N_{1}$ and $N_{5}$}

We first explain the strategy of reducing the estimate of the fundamental solution to three steps of Picard iterations.

\section{Step 1. (The strategy.)}

Denote the ODE by $d Y / d t=\Lambda(t) Y$ with the initial condition $Y(0)=\operatorname{Id}_{10}$. Using the Picard iteration, the solution is

$$
\begin{aligned}
Y(t) & =\mathrm{Id}+\int_{0}^{t} \Lambda \cdot Y(s) d s=\mathrm{Id}+\int_{0}^{t} \Lambda d t+\int_{0}^{t} \Lambda\left(\int_{0}^{s} \Lambda(\tau) d \tau\right) d s+\ldots \\
: & =\mathrm{Id}+I_{1}(t)+I_{2}(t)+\ldots
\end{aligned}
$$

where $I_{i}$ is the $i$ th iterated integral. We will show that

$$
Z(t)=\operatorname{Id}+c_{1} I_{1}(t)+c_{2} I_{2}(t)+\ldots+c_{n} I_{n}(t),
$$

with properly chosen $c_{n}=c_{n-1}=\ldots=c_{3}>c_{2}>c_{1}>1$, satisfies the inequality $Z^{\prime} \geqslant \Lambda(t) Z(t)$, or equivalently

$$
\begin{aligned}
\operatorname{Id}+c_{1} I_{1}(t)+\ldots+c_{n} I_{n}(t)=Z(t) & \geqslant \operatorname{Id}+\int_{0}^{t} \Lambda(s) Z(s) d s \\
& \geqslant \operatorname{Id}+I_{1}(t)+c_{1} I_{2}(t)+c_{2} I_{3}(t)+\ldots+c_{n} I_{n+1}(t), \\
c_{n} I_{n+1}(t) & \leqslant\left(c_{1}-1\right) I_{1}(t)+\left(c_{2}-c_{1}\right) I_{2}(t)+\left(c_{3}-c_{2}\right) I_{3}(t) .
\end{aligned}
$$

Then, by the Gronwall inequality, we get that $Y(t) \leqslant Z(t)$.

\section{Step 2. (Checking (7.11).)}

We next show how to compute the matrix products. The following observations allow us to reduce (7.11) to computing products of constant matrices, which simplifies the calculation significantly. In $\mathrm{u}, \mathrm{v}$ and $\mathrm{w}$, we replace $\mu /\left(\ell_{4}^{3}+1\right)$ by $\mu /\left|\ell_{4}\right|^{3}$ with $\ell_{4}$ lying between 1 and $O(\chi)$. Recall that $\mu /\left|\ell_{4}\right|^{3}$ is the correct bound of terms of the form $\mu\left|x_{3}\right| /\left|x_{4}\right|^{3}$ in Lemma 6.6 , while $\mu /\left(\ell_{4}^{3}+1\right)$ was used to show that the denominator cannot be zero.

For $N_{1}$, we pick a small constant $\varepsilon_{0}$ which is independent of $\mu$ and $\chi$, so that

$$
\int_{\ell_{4}^{\mathrm{i}}}^{\ell_{4}^{\mathrm{i}}+\varepsilon_{0}} \frac{\mu}{\ell_{4}^{3}} d \ell_{4}=\frac{\mu \varepsilon_{0}}{\left(\ell_{4}^{\mathrm{i}}\right)^{3}}+O\left(\varepsilon_{0}^{2}\right)
$$

where $\ell_{4}^{\mathrm{i}}=O(1) \neq 0$ is the initial $\ell_{4}$. Inequality (7.11) holds for $\ell_{4} \in\left[\ell_{4}^{\mathrm{i}}, \ell_{4}^{\mathrm{i}}+\varepsilon_{0}\right]$ for $\varepsilon_{0}$ small enough. For $\ell_{4} \geqslant \ell_{4}^{\mathrm{i}}+\varepsilon_{0}$, we have

$$
\int_{\ell_{4}^{\mathrm{i}}}^{\ell_{4}} \frac{\mu}{s^{3}} d s=\frac{\mu}{2\left(\ell_{4}^{\mathrm{i}}\right)^{2}}-\frac{\mu}{2\left(\ell_{4}\right)^{2}}=O(\mu)
$$


as $\ell_{4} \rightarrow \infty$ and $\mu \rightarrow 0$. So, we replace all the integrals $\int_{\ell_{4}^{\mathrm{i}}}^{\ell_{4}}\left(\mu / s^{3}\right) d s$ by $\mu$ in the sense of " $\sim$ ". After integration in (7.11), there are no terms of the form $1 / \ell_{4}^{k}, k>0$.

Notice that we can decompose right matrix in Lemma 7.3 as $K+\mu B / \ell_{4}^{3}$, where $K$ and $B$ do not depend on $\ell_{4}$, and $K$ is exactly the same as in (7.8). We have

$$
\begin{aligned}
& I_{1}\left(\ell_{4}\right) \lesssim \ell_{4} K+\mu B, \\
& I_{2}\left(\ell_{4}\right) \lesssim \ell_{4}^{2} K^{2}+\mu \ell_{4} K B+\mu B K+\mu^{2} B^{2}, \\
& I_{3}\left(\ell_{4}\right) \lesssim \ell_{4}^{3} K^{3}+\mu \ell_{4}^{2} K^{2} B+\mu \ell_{4} K B K+\mu^{2} \ell_{4} K B^{2} \\
& \quad+\mu \ln \ell_{4} B K^{2}+\mu^{2}\left(B K B+B^{2} K\right)+\mu^{3} B^{3} .
\end{aligned}
$$

To simplify the the proof, we note that $I_{3}\left(\ell_{4}\right)$ is bounded by

$$
\frac{\ell_{4}^{3} K^{3}}{3 !}+\varepsilon I_{1}\left(\ell_{4}\right)+\varepsilon I_{2}\left(\ell_{4}\right),
$$

where $\varepsilon$ can be chosen to be arbitrarily small, provided $\mu$ and $1 / \chi$ are small enough. So, $I_{4}\left(\ell_{4}\right)$ is bounded by

$$
\int_{1}^{\ell_{4}}\left(K+\frac{\mu}{t^{3}} B\right)\left(t^{3} K^{3}+\varepsilon I_{1}(t)+\varepsilon I_{2}(t)\right) d t \leqslant \frac{\ell_{4}^{4} K^{4}}{4 !}+\mu \ell_{4} B K^{3}+\varepsilon I_{2}\left(\ell_{4}\right)+\varepsilon I_{3}\left(\ell_{4}\right) .
$$

It turns out that we have $\mu \chi B K^{3} \ll \chi^{4} K^{4}$, so we get

$$
I_{4}\left(\ell_{4}\right) \leqslant \frac{1+\varepsilon}{4 !} \ell_{4}^{4} K^{4}+\varepsilon I_{2}\left(\ell_{4}\right)+\varepsilon I_{3}\left(\ell_{4}\right)
$$

Inductively, we have

$$
I_{n}\left(\ell_{4}\right) \leqslant C_{n} \ell_{4}^{n} K^{n}+\varepsilon I_{n-2}\left(\ell_{4}\right)+\varepsilon I_{n-1}\left(\ell_{4}\right),
$$

where $C_{n}$ satisfies

$$
C_{n+1} \leqslant\left(\frac{1}{n+1}+\frac{\varepsilon}{n-2}\right) C_{n} .
$$

We can bound $C_{n} \leqslant C(1+2 \varepsilon)^{n} / n$ !. Moreover, by $\chi^{4} K^{4} \leqslant C \chi^{3} K^{3}$, we get

$$
\chi^{n} K^{n} \leqslant C^{n-3} \chi^{3} K^{3},
$$

where $C$ is independent of $\mu$ or $\chi$. So, we get

$$
I_{n}(\chi) \leqslant C \frac{(C(1+2 \varepsilon))^{n}}{n !} \chi^{3} K^{3}+\varepsilon I_{n-2}(\chi)+\varepsilon I_{n-1}(\chi) .
$$


By applying (7.12) recursively, we can bound

$$
I_{n}(\chi) \leqslant C \frac{(C(1+2 \varepsilon))^{n}}{n !} \chi^{3} K^{3}+O(\varepsilon) \chi^{3} K^{3}+O(\varepsilon) I_{1}(\chi)+O(\varepsilon) I_{2}(\chi),
$$

where the $O$ s depend on $n$ but not on $\mu, \chi$. We choose $n$ (independent of $\mu$ and $\chi$ ) so that $C(C(1+2 \varepsilon))^{n} / n$ ! is smaller than $\frac{1}{100}$. We then choose $\mu$, without changing $n$, so that all the $O(\varepsilon) s$ are smaller than $\frac{1}{100}$. We can replace $\chi^{3} K^{3}$ by $I_{3}(\chi)$, because $\frac{1}{6} \chi^{3} K^{3}<I_{3}(\chi)$. Then, we see that (7.11) is satisfied if we choose $c_{1}=2, c_{2}=3$ and $c_{3}=4$.

For $N_{5}$, we integrate $\ell_{4}$ from $O(\chi)$ to $O(1)$, using only $1 / \chi$ in $\mathrm{w}\left(\ell_{4}\right)$ when doing integration since its integral dominates the other term. Again, we can decompose the right matrix in Lemma 7.3 as $K+\mu B / \ell_{4}^{3}$, whose integration for $\ell_{4}$ from $\frac{1}{2} \chi$ to 1 is $\sim\left(\frac{1}{2} \chi-\ell_{4}\right) K+\mu B / \ell_{4}^{2}$. We have

$$
\begin{aligned}
& I_{1}\left(\ell_{4}\right) \lesssim\left(\chi-\ell_{4}\right) K+\frac{\mu}{\ell_{4}^{2}} B \\
& I_{2}\left(\ell_{4}\right) \lesssim\left(\chi-\ell_{4}\right)^{2} K^{2}+\frac{\mu}{\ell_{4}} K B+\mu \frac{\left(\chi-\ell_{4}\right)}{\ell_{4}^{2}} B K+\frac{\mu^{2}}{\ell_{4}^{4}} B^{2}, \\
& I_{3}\left(\ell_{4}\right) \lesssim\left(\chi-\ell_{4}\right)^{3} K^{3}+\mu \ln \frac{\ell_{4}}{\chi} K^{2} B+\mu\left(\frac{\chi}{\ell_{4}}-\log \ell_{4}\right) K B K+\frac{\mu^{2}}{\ell_{4}^{3}} K B^{2} \\
& \quad+\mu \frac{\left(\chi-\ell_{4}\right)^{2}}{\ell_{4}^{2}} B K^{2}+\frac{\mu^{2}}{\ell_{4}^{3}} B K B+\mu^{2} \frac{\chi-\ell_{4}}{\ell_{4}^{4}} B^{2} K+\frac{\mu^{3}}{\ell_{4}^{6}} B^{3} .
\end{aligned}
$$

We next prove that three steps of Picard iterations give the correct estimate of the fundamental solution. It can be verified that

$$
I_{3}\left(\ell_{4}\right) \leqslant \frac{\left(\chi-\ell_{4}\right)^{3} K^{3}}{3 !}+\mu \frac{\left(\chi-\ell_{4}\right)^{2}}{\ell_{4}^{2}} B K^{2}+\varepsilon\left(I_{1}\left(\ell_{4}\right)+I_{2}\left(\ell_{4}\right)\right) .
$$

In other words, the new contributions from $I_{3}$ come mainly from $K^{3}$ and $B K^{2}$. Moreover, we have

$$
\frac{\mu^{2} B^{2}}{\ell_{4}^{4}}+\mu \frac{\chi-\ell_{4}}{\ell_{4}} K B K \leqslant \varepsilon\left(\left(\chi-\ell_{4}\right) K+\frac{\mu B}{\ell_{4}^{2}}+\left(\chi-\ell_{4}\right)^{2} K^{2}\right) .
$$

This inequality allows us to remove higher powers of $B$ and to keep only the $K^{j}$ and $B K^{j}$ terms.

Next, by splitting the integral into integrals over $\left[1, \frac{1}{2} \chi\right]$ and $\left[\frac{1}{2} \chi, \chi\right]$, we have, for $t \in[1, \chi]$,

$$
\int_{t}^{\chi} \frac{(\chi-s)^{n}}{s^{k}} d s \leqslant 100 \cdot 2^{n} t^{-k+1}(\chi-t)^{n}
$$

for $n \geqslant 1$ and $k=2,3,4,5$. 
Let us now consider $I_{4}$ :

$$
\begin{aligned}
& I_{4}\left(\ell_{4}\right) \\
& \leqslant \int_{\ell_{4}}^{\chi}\left(K+\frac{\mu}{t^{3}} B\right)\left(\frac{(\chi-t)^{3} K^{3}}{3 !}+\mu \frac{(\chi-t)^{2}}{t^{2}} B K^{2}+\varepsilon\left(I_{1}(t)+I_{2}(t)\right)\right) d t \\
& \leqslant \frac{1}{4 !}\left(\chi-\ell_{4}\right)^{4} K^{4}+100 \cdot 2^{3} \frac{1}{3 !} \mu \frac{\left(\chi-\ell_{4}\right)^{3}}{\ell_{4}^{2}} B K^{3}+100 \cdot 2^{2} \mu \frac{\left(\chi-\ell_{4}\right)^{2}}{\ell_{4}} K B K^{2} \\
&+100 \cdot 2^{2} \mu^{2} \frac{\left(\chi-\ell_{4}\right)^{2}}{\ell_{4}^{4}} B^{2} K^{2}+\varepsilon\left(I_{2}\left(\ell_{4}\right)+I_{3}\left(\ell_{4}\right)\right) \\
& \leqslant \frac{1}{4 !}\left(\chi-\ell_{4}\right)^{4} K^{4}+100 \cdot 2^{3} \frac{1}{3 !} \mu \frac{\left(\chi-\ell_{4}\right)^{3}}{\ell_{4}^{2}} B K^{3}+\varepsilon\left(I_{2}\left(\ell_{4}\right)+I_{3}\left(\ell_{4}\right)\right) \\
&+100 \cdot 2^{3} \varepsilon\left(\left(\chi-\ell_{4}\right)^{2} K^{2}+\left(\chi-\ell_{4}\right)^{3} K^{3}+\left(\chi-\ell_{4}\right)^{4} K^{4}+\mu \frac{\left(\chi-\ell_{4}\right)}{\ell_{4}^{2}} B K+\mu \frac{\left(\chi-\ell_{4}\right)^{2}}{\ell_{4}^{2}} B K^{2}\right),
\end{aligned}
$$

where, in the last $\leqslant$, we use (7.13). Inductively, we get

$$
\begin{aligned}
& I_{n}\left(\ell_{4}\right) \leqslant \frac{C^{n}}{n !}\left(\chi-\ell_{4}\right)^{n} K^{n}+\frac{C^{n}}{(n-1) !} \mu \frac{\left(\chi-\ell_{4}\right)^{n-1}}{\ell_{4}^{2}} B K^{n-1}+\varepsilon\left(I_{n-2}\left(\ell_{4}\right)+I_{n-1}\left(\ell_{4}\right)\right) \\
&+\varepsilon C^{n}\left(\sum_{j=1}^{n}\left(\chi-\ell_{4}\right)^{j} K^{j}\right)+(C \varepsilon)^{n-2} \mu \frac{B}{\ell_{4}^{2}}\left(\sum_{j=1}^{n-2}\left(\chi-\ell_{4}\right)^{j} K^{j}\right),
\end{aligned}
$$

where $(C \varepsilon)^{n-2}$ appears since each application of (7.13) gives rise to a multiple of $C \varepsilon$. Further argument is similar to the $N_{1}$ case.

\section{Estimates of the boundary contribution}

In this section, we work on all the boundary contributions (see Definition 7.1 and equation (7.1)) for the maps (I), (III) and (V).

\subsection{Boundary contribution for (I)}

Computation of matrix (I) in Proposition 5.2. By (7.1), (I) is a product of three matrices (7.1) and we already know the matrix $N_{1}$, i.e. the solution of the variational equation. It remains to work out the two matrices for boundary contributions. The expression for $x_{4, \|}^{R}$ is the following (see Appendix A):

$$
x_{4, \|}^{R}=-\left(\cos g_{4}\right) L_{4}^{2}\left(\cosh u_{4}-e_{4}\right)+\left(\sin g_{4}\right) L_{4} G_{4} \sinh u_{4} .
$$


For fixed $x_{4, \|}^{R}=-\frac{1}{2} \chi$ or $x_{4, \|}^{R}=-2$, we can solve $\ell_{4}$ as a function of $L_{4}, G_{4}$ and $g_{4}$. The bounds for $L_{4}$ and $G_{4}$ have been obtained in Lemma 6.4, 6.6 (a) and 6.7. So, we get the following using the implicit function theorem and Lemma A.3:

$$
\begin{aligned}
& \left(\frac{\partial \ell_{4}}{\partial L_{4}}, \frac{\partial \ell_{4}}{\partial G_{4}}, \frac{\partial \ell_{4}}{\partial g_{4}}\right)=-\left(\frac{\partial x_{4, \|}}{\partial \ell_{4}}\right)^{-1}\left(\frac{\partial x_{4, \|}}{\partial L_{4}}, \frac{\partial x_{4, \|}}{\partial G_{4}}, \frac{\partial x_{4, \|}}{\partial g_{4}}\right), \\
& \left.\left(\frac{\partial \ell_{4}}{\partial L_{4}}, \frac{\partial \ell_{4}}{\partial G_{4}}, \frac{\partial \ell_{4}}{\partial g_{4}}\right)^{R}\right|_{x_{4, \|}^{R}=-\chi / 2} \lesssim(\chi, 1,1), \\
& \left.\left(\frac{\partial \ell_{4}}{\partial L_{4}}, \frac{\partial \ell_{4}}{\partial G_{4}}, \frac{\partial \ell_{4}}{\partial g_{4}}\right)^{R}\right|_{x_{4, \|}^{R}=-2} \lesssim(1,1,1) .
\end{aligned}
$$

Note that $\ell_{4}$ depends on all other variables including $L_{4}$, so, using Corollary 6.10 and Lemma 7.2, we obtain, for the section $x_{4, \|}^{R}=-2$,

$$
\begin{aligned}
\left.\left(\frac{\partial \ell_{4}}{\partial \mathcal{V}}\right)^{R}\right|_{x_{4, \|}^{R}=-2} & \lesssim\left(1, \mu, \mu, \mu ; \frac{1}{\mu \chi^{2}}, \frac{1}{\chi^{3}}, \mu, \frac{\mu}{\chi} ; 1,1\right)_{1 \times 10}, \\
\left.\mathcal{F}^{R}\right|_{x_{4, \|}^{R}=-2} & \lesssim\left(\mu, 1, \mu, \mu ; \mu, \frac{\mu}{\chi}, \frac{1}{\mu \chi^{2}}, \frac{1}{\chi^{3}} ; \mu, \mu\right)_{1 \times 10}^{T},
\end{aligned}
$$

and, for the section $x_{4, \|}^{R}=-\frac{1}{2} \chi$,

$$
\begin{aligned}
\left.\left(\frac{\partial \ell_{4}}{\partial \mathcal{V}}\right)^{R}\right|_{x_{4, \|}^{R}=-\chi / 2} & \lesssim\left(\chi, \frac{1}{\chi^{2}}, \frac{1}{\chi^{2}}, \frac{1}{\chi^{2}} ; \frac{1}{\mu \chi}, \frac{1}{\chi^{2}}, \mu \chi, \mu ; 1,1\right)_{1 \times 10}, \\
\left.\mathcal{F}^{R}\right|_{x_{4, \|}^{R}=-\chi / 2} & \lesssim\left(\frac{1}{\chi^{3}}, 1, \frac{1}{\chi^{3}}, \frac{1}{\chi^{3}} ; \mu, \frac{\mu}{\chi}, \frac{1}{\mu \chi^{2}}, \frac{1}{\chi^{3}} ; \frac{1}{\chi^{2}}, \frac{1}{\chi^{2}}\right)_{1 \times 10}^{T},
\end{aligned}
$$

where the two entries in bold font are estimates in the sense of $\sim$ rather than $O$. The 1 entry in $\left.\mathcal{F}^{R}\right|_{x_{4, \|}^{R}=-\chi / 2}$ is already established in Corollary 6.10. To get the $\chi$ entry in

$$
\left.\left(\frac{\partial \ell_{4}}{\partial \mathcal{V}}\right)^{R}\right|_{x_{4, \|}^{R}=-\chi / 2},
$$

we use the $(1,1)$ and $(1,2)$ entries in $\mathcal{D}$ from (A.7). The result is

$$
-\frac{\partial x_{4, \|}}{\partial L_{4}}\left(\frac{\partial x_{4, \|}}{\partial \ell_{4}}\right)^{-1} \simeq-\frac{2 \ell_{4}}{L_{4}} \simeq \frac{\chi}{L_{4}^{3}}
$$

where the last equality is obtained by setting $Q_{\|}=-\frac{1}{2} \chi$ in (A.5). In this case $u>0$ and $\ell_{4}<0$. Denote

$$
\begin{aligned}
& l:=\left(1, \frac{1}{\chi^{3}}, \frac{1}{\chi^{3}}, \frac{1}{\chi^{3}} ; \frac{1}{\mu \chi^{2}}, \frac{1}{\chi^{3}}, \mu, \frac{\mu}{\chi} ; \frac{1}{\chi}, \frac{1}{\chi}\right)_{1 \times 10}, \\
& u:=\left(\frac{1}{\chi^{3}}, \mathbf{1}, \frac{1}{\chi^{3}}, \frac{1}{\chi^{3}} ; \mu, \frac{\mu}{\chi}, \frac{1}{\mu \chi^{2}}, \frac{1}{\chi^{3}} ; \frac{1}{\chi^{2}}, \frac{1}{\chi^{2}}\right)_{1 \times 10}^{T} .
\end{aligned}
$$


Then, (8.4) gives

$$
\left.\frac{1}{\chi}\left(\frac{\partial \ell_{4}}{\partial \mathcal{V}}\right)^{R}\right|_{x_{4, \|}^{R}=-\chi / 2} \lesssim l \text { and }\left.\mathcal{F}^{R}\right|_{x_{4, \|}^{R}=-\chi / 2} \lesssim u
$$

Define

$$
\begin{gathered}
u_{1}^{\mathrm{i}}=\left.\mathcal{F}^{R}\right|_{x_{4, \|}^{R}=-2}, \quad l_{1}^{\mathrm{i}}=\left.\left(\frac{\partial \ell_{4}}{\partial \mathcal{V}}\right)^{R}\right|_{x_{4, \|}^{R}=-2}, \\
u_{1}^{\mathrm{f}}=\left.(1+O(\mu)) \mathcal{F}^{R}\right|_{x_{4, \|}^{R}=-\chi / 2} \lesssim u, \quad l_{1}^{\mathrm{f}}=\left.\frac{1}{\chi}\left(\frac{\partial \ell_{4}}{\partial \mathcal{V}}\right)^{R}\right|_{x_{4, \|}^{R}=-\chi / 2} \lesssim l,
\end{gathered}
$$

where the inequalities follow from (8.6) and (8.4). Then,

$$
(\mathrm{I})=\left(\mathrm{Id}-\mathcal{F}^{R} \otimes\left(\frac{\partial \ell_{4}}{\partial \mathcal{V}}\right)^{R}\right)^{-1} N_{1}\left(\mathrm{Id}-u_{1}^{\mathrm{i}} \otimes l_{1}^{\mathrm{i}}\right),
$$

as claimed in Proposition 5.2. To invert

$$
\mathrm{Id}-\mathcal{F}^{R} \otimes\left(\frac{\partial \ell_{4}}{\partial \mathcal{V}}\right)^{R}
$$

we use (7.2) and verify that

$$
\left(\frac{\partial \ell_{4}}{\partial \mathcal{V}}\right)^{R} \cdot \mathcal{F}^{R}=O(\mu)
$$

So, we get (I) as claimed in Proposition 5.2.

Finally, we show the $\beta$ rotation of the section $\left\{x_{4, \|}^{R}=-2, v_{4, \|}^{R}>0\right\}$ to the section $\left\{\left(\operatorname{Rot}(-\beta) \cdot x_{4}\right)_{\|}^{R}=-2, v_{4, \|}^{R}>0\right\}$, after applying $\mathcal{R}$ in Definition 2.6 is negligible. Instead of $(8.1)$, we need to use the expression $(\cos \beta) x_{4, \|}^{R}-(\sin \beta) x_{4, \perp}^{R}=-2$, and convert $x_{4}^{R}$ into Delaunay variables. Since we have $\ell_{4}^{R}=O(1)$ here, and $\beta=O(\mu / \chi)$ since $x_{1} \in \mathcal{S}_{\mu \widehat{C}}$, we get a correction of order $O(\mu / \chi) \cdot\left(\partial \ell_{4} / \partial \mathcal{V}\right)^{R}$ to $\left(\partial \ell_{4} / \partial \mathcal{V}\right)^{R}$ in $(8.3)$, which is negligible.

\subsection{Boundary contribution for (III)}

Computation of matrix (III) in Proposition 5.2. For the matrix (III), the solution for the variational equation is given by $N_{3}$. We only need to work out the two boundary terms on the sections $\left\{x_{4, \|}^{R}=-\frac{1}{2} \chi, v_{4, \|}^{R}<0\right\}$ and $\left\{x_{4, \|}^{L}=\frac{1}{2} \chi, v_{4, \|}^{L}>0\right\}$. In (7.1), the variables $\mathcal{V}^{\mathrm{i}}$ and $\mathcal{V}^{\mathrm{f}}$ should carry superscript $L$ for matrix (III), since we did not compose a coordinate change between the left and right variables in (7.1). However, the section $\left\{x_{4, \|}^{R}=-\frac{\chi}{2}, v_{4, \|}^{R}<0\right\}$ is defined using variables with superscript $R$, so we first need to 
express it using left variables. We use the matrix $R \cdot L^{-1}$ to get $\mathcal{X}^{R}=R \cdot L^{-1} \mathcal{X}^{L}$. This implies

$$
\begin{aligned}
x_{4, \|}^{R} & =x_{1, \|}^{L}+\frac{1}{1+\mu} x_{4, \|}^{L} \\
& =x_{1, \|}^{L}+\frac{1}{1+\mu}\left(\left(\cos g_{4}\right) L_{4}^{2}\left(\cosh u_{4}-e_{4}\right)-\left(\sin g_{4}\right) L_{4} G_{4} \sinh u_{4}\right)=-\frac{\chi}{2} .
\end{aligned}
$$

So, we get the following using the implicit function theorem and Lemma A.3 from the appendix:

$$
\begin{aligned}
& \left(\frac{\partial \ell_{4}}{\partial L_{4}}, \frac{\partial \ell_{4}}{\partial G_{4}}, \frac{\partial \ell_{4}}{\partial g_{4}}, \frac{\partial \ell_{4}}{\partial x_{1, \|}}\right)^{L}=-\left(\frac{\partial x_{4, \|}^{L}}{\partial \ell_{4}}\right)^{-1}\left(\frac{\partial x_{4, \|}}{\partial L_{4}}, \frac{\partial x_{4, \|}}{\partial G_{4}}, \frac{\partial x_{4, \|}}{\partial g_{4}},-(1+\mu)\right)^{L}, \\
& \left.\left(\frac{\partial \ell_{4}}{\partial L_{4}}, \frac{\partial \ell_{4}}{\partial G_{4}}, \frac{\partial \ell_{4}}{\partial g_{4}}, \frac{\partial \ell_{4}}{\partial x_{1, \|}}\right)^{L}\right|_{x_{4, \|}^{R}=-\chi / 2} \lesssim(\chi, 1,1,1) .
\end{aligned}
$$

Using Corollary 6.10 and Lemma 7.2, we obtain

$$
\begin{aligned}
\left.\left(\frac{\partial \ell_{4}}{\partial \mathcal{V}}\right)^{L}\right|_{x_{4, \|}^{R}=-\chi / 2} & \lesssim\left(\chi, \frac{1}{\chi^{2}}, \frac{1}{\chi^{2}}, \frac{1}{\chi^{2}} ; 1, \frac{1}{\chi^{2}}, \mu \chi, \mu ; 1,1\right)_{1 \times 10}, \\
\left.\mathcal{F}^{L}\right|_{x_{4, \|}^{R}=-\chi / 2} & \lesssim\left(\frac{1}{\chi^{3}}, 1, \frac{1}{\chi^{3}}, \frac{1}{\chi^{3}} ; \mu, \frac{\mu}{\chi}, \frac{1}{\mu \chi^{2}}, \frac{1}{\chi^{3}} ; \frac{1}{\chi^{2}}, \frac{1}{\chi^{2}}\right)_{1 \times 10}^{T},
\end{aligned}
$$

where 1 and $\chi$ are estimates in the sense of $\sim$, having the same values as that in (8.4). Denote

$$
l^{\prime}:=\left(1, \frac{1}{\chi^{3}}, \frac{1}{\chi^{3}}, \frac{1}{\chi^{3}} ; \frac{1}{\chi}, \frac{1}{\chi^{3}}, \mu, \frac{\mu}{\chi} ; \frac{1}{\chi}, \frac{1}{\chi}\right)_{1 \times 10},
$$

which is different from $l$ in its fifth entry. Then (8.9) becomes

$$
\left.\frac{1}{\chi}\left(\frac{\partial \ell_{4}}{\partial \mathcal{V}}\right)^{L}\right|_{x_{4, \|}^{R}=-\chi / 2} \lesssim l^{\prime} \quad \text { and }\left.\quad \mathcal{F}^{L}\right|_{x_{4, \|}^{R}=-\chi / 2} \lesssim u .
$$

For the section $\left\{x_{4, \|}^{L}=\frac{1}{2} \chi, v_{4, \|}^{L}>0\right\}$, the estimate is exactly the same as the case $\left\{x_{4, \|}^{R}=-\frac{1}{2} \chi, v_{4, \|}^{L}<0\right\}$ in (I), i.e. $u_{1}^{\mathrm{f}}$ and $l_{1}^{\mathrm{f}}$, and we get the same result as (8.7):

$$
u_{3}^{\mathrm{f}}:=\left.\mathcal{F}^{L}\right|_{x_{4, \|}^{L}=\frac{\chi}{2}} \lesssim u \quad \text { and } \quad l_{3}^{\mathrm{f}}:=\left.\frac{1}{\chi}\left(\frac{\partial \ell_{4}}{\partial \mathcal{V}}\right)^{L}\right|_{x_{4, \|}^{L}=\chi / 2} \lesssim l .
$$

However, we note that the $\chi$ entries in

$$
\left.\left(\frac{\partial \ell_{4}}{\partial \mathcal{V}}\right)^{L}\right|_{x_{4, \|}^{R}=-\chi / 2} \text { and }\left.\left(\frac{\partial \ell_{4}}{\partial \mathcal{V}}\right)^{L}\right|_{x_{4, \|}^{L}=\chi / 2}
$$


have the same expression $-2 \ell_{4} / L_{4}$, by the same calculation as in $\S 8.1$. So we get that the former is actually $-\chi$, while the latter is $\chi$. This proves the sign differences of $l_{3}^{\mathrm{i}}$ and $l_{3}^{\mathrm{f}}$ in Proposition $5.2(\mathrm{a} .2)$.

We obtain the matrix $(\mathrm{III})=\left(\operatorname{Id}+\chi u_{3}^{\mathrm{f}} \otimes l_{3}^{\mathrm{f}}\right) N_{3}\left(\mathrm{Id}-\chi u_{3}^{\mathrm{i}} \otimes l_{3}^{\mathrm{i}}\right)$ in Proposition 5.2 , by defining

$$
l_{3}^{\mathrm{i}}:=\left.\frac{1}{\chi}\left(\frac{\partial \ell_{4}}{\partial \mathcal{V}}\right)^{L}\right|_{x_{4, \|}^{R}=-\chi / 2} \lesssim l^{\prime} \quad \text { and } \quad u_{3}^{\mathrm{i}}:=\left.\mathcal{F}^{L}\right|_{x_{4, \|}^{R}=-\chi / 2} \lesssim u,
$$

where the inequalities follow from (8.11).

\subsection{Boundary contribution for (V)}

Computation of matrix ( $V$ ) in Proposition 5.2. For the matrix (V), the solution of the variational equation is given by $N_{5}$. We only need to get two boundary contributions. Notice that the section $\left\{x_{4, \|}^{L}=\frac{1}{2} \chi, v_{4, \|}^{L}>0\right\}$ is defined using left variables. However, we need to express the boundary contributions in (7.1). The estimate is exactly the same as that for the section $\left\{x_{4, \|}^{R}=-\frac{1}{2} \chi, v_{4, \|}^{R}<0\right\}$ of (III), i.e. $u_{3}^{\mathrm{i}}$ and $l_{3}^{\mathrm{i}}$, though this time we need to use $\mathcal{X}^{L}=L \cdot R^{-1} \mathcal{X}^{R}$. We get the same result as (8.13):

$$
u_{5}^{\mathrm{i}}:=\left.\mathcal{F}^{R}\right|_{x_{4, \|}^{L}=\frac{\chi}{2}} \lesssim u \quad \text { and } \quad l_{5}^{\mathrm{i}}:=\left.\frac{1}{\chi}\left(\frac{\partial \ell_{4}}{\partial \mathcal{V}}\right)^{R}\right|_{x_{4, \|}^{L}=\frac{\chi}{2}} \lesssim l^{\prime}
$$

For the section $\left\{x_{4, \|}^{R}=-2\right\}$, the estimate is exactly the same as the estimate in the $\left\{x_{4, \|}^{R}=-2\right\}$ case of $(\mathrm{I})$, i.e. $u_{1}^{\mathrm{i}}$ and $l_{1}^{\mathrm{i}}$ in (8.7). Defining

$$
u_{5}^{\mathrm{f}}:=\left.\mathcal{F}^{R}\right|_{x_{4, \|}^{R}=-2} \quad \text { and } \quad l_{5}^{\mathrm{f}}:=\left.\left(\frac{\partial \ell_{4}}{\partial \mathcal{V}}\right)^{R}\right|_{x_{4, \|}^{R}=-2}
$$

we get

$$
(\mathrm{V})=\left(\mathrm{Id}+\chi u_{5}^{\mathrm{f}} \otimes l_{5}^{\mathrm{f}}\right) N_{5}\left(\mathrm{Id}-u_{1}^{\mathrm{i}} \otimes l_{5}^{\mathrm{i}}\right),
$$

as claimed in Proposition 5.2. The signs of $l_{5}^{\mathrm{i}}$ and $l_{5}^{\mathrm{f}}$ are analyzed in the same way as the cases (I) and (III).

\section{Estimates of the matrices (II) and (IV) for switching foci}

In this section, we study the matrices (II) and (IV) in Proposition 5.2. 


\subsection{A simplifying computation}

We start with a formal calculation, which liberates us from calculating the $\mathcal{V}_{3}$ part. Both $R \cdot L^{-1}$ are $L \cdot R^{-1}$ can be represented as

$$
\left[\begin{array}{cc}
\operatorname{Id}_{4 \times 4} & 0 \\
0 & T_{\mu}
\end{array}\right]
$$

for a $8 \times 8$ matrix $T_{\mu}$. We need to multiply $\partial \mathcal{V}^{L} / \partial \mathcal{X}^{L}$ on the left and $\partial \mathcal{X}^{R} / \partial \mathcal{V}^{R}$ on the right to get (II) $=\partial \mathcal{V}^{L} / \partial \mathcal{V}^{R}$ as follows:

$$
\begin{aligned}
& \frac{\partial \mathcal{V}^{L}}{\partial \mathcal{X}^{L}} L \cdot R^{-1} \frac{\partial \mathcal{X}^{R}}{\partial \mathcal{V}^{R}} \\
& =\left[\begin{array}{cc}
\frac{\partial \mathcal{V}_{3}}{\partial \mathcal{X}_{3}} & 0 \\
0 & \frac{\partial\left(\mathcal{V}_{1}, \mathcal{V}_{4}\right)}{\partial\left(\mathcal{X}_{1}, \mathcal{X}_{4}\right)}
\end{array}\right]_{10 \times 12}^{L}\left[\begin{array}{cc}
\operatorname{Id}_{4 \times 4} & 0 \\
0 & T_{\mu}
\end{array}\right]_{12 \times 12}\left[\begin{array}{cc}
\frac{\partial \mathcal{X}_{3}}{\partial \mathcal{V}_{3}} & 0 \\
\frac{\partial\left(\mathcal{X}_{1}, \mathcal{X}_{4}\right)}{\partial \mathcal{V}_{3}} & \frac{\partial\left(\mathcal{X}_{1}, \mathcal{X}_{4}\right)}{\partial\left(\mathcal{V}_{1}, \mathcal{V}_{4}\right)}
\end{array}\right]_{12 \times 10}^{R} \\
& =\left[\begin{array}{cc}
\operatorname{Id} & 0 \\
0 & \frac{\partial\left(\mathcal{V}_{1}, \mathcal{V}_{4}\right)}{\partial\left(\mathcal{X}_{1}, \mathcal{X}_{4}\right)}
\end{array}\right]_{10 \times 12}^{L}\left[\begin{array}{cc}
\operatorname{Id} & 0 \\
0 & T_{\mu}
\end{array}\right]_{12 \times 12}\left[\begin{array}{cc}
\frac{\partial\left(\mathcal{X}_{1}, \mathcal{X}_{4}\right)}{\partial \mathcal{V}_{3}} & \frac{\partial\left(\mathcal{X}_{1}, \mathcal{X}_{4}\right)}{\partial\left(\mathcal{V}_{1}, \mathcal{V}_{4}\right)}
\end{array}\right]_{12 \times 10}^{R} .
\end{aligned}
$$

We have the same calculation for

$$
(\mathrm{IV})=\frac{\partial \mathcal{V}^{R}}{\partial \mathcal{V}^{L}}=\frac{\partial \mathcal{V}^{R}}{\partial \mathcal{X}^{R}} R \cdot L^{-1} \frac{\partial \mathcal{X}^{L}}{\partial \mathcal{V}^{L}}
$$

In the following, we only need to figure out the matrices

$$
\frac{\partial\left(\mathcal{X}_{1}, \mathcal{X}_{4}\right)}{\partial\left(\mathcal{V}_{3}, \mathcal{V}_{1}, \mathcal{V}_{4}\right)} \quad \text { and } \quad \frac{\partial\left(\mathcal{V}_{1}, \mathcal{V}_{4}\right)}{\partial\left(\mathcal{X}_{1}, \mathcal{X}_{4}\right)}
$$

\subsection{From Delaunay to Cartesian coordinates}

In this section, we compute

$$
\frac{\partial\left(\mathcal{X}_{1} ; \mathcal{X}_{4}\right)}{\partial\left(\mathcal{V}_{3}, \mathcal{V}_{1}, \mathcal{V}_{4}\right)}=\frac{\partial\left(x_{1}, v_{1}, x_{4}, v_{4}\right)}{\partial\left(L_{3}, \ell_{3}, G_{3}, g_{3}, x_{1}, v_{1}, G_{4}, g_{4}\right)}
$$

This computation is restricted to the section $\left\{x_{4, \|}^{R}=-\frac{1}{2} \chi\right\}$ for matrix (II), and to the section $\left\{x_{4, \|}^{L}=\frac{1}{2} \chi\right\}$ for matrix (IV). The key observation to obtain the tensor structure of the following sublemma is explained in Remark A.4(2). 
Sublemma 9.1. Assume AG. Then, the following holds.

(a) On the section $\left\{x_{4, \|}^{R}=-\frac{1}{2} \chi\right\}$, the matrix $\partial\left(\mathcal{X}_{1}, \mathcal{X}_{4}\right)^{R} / \partial \mathcal{V}^{R}$ in (9.1) is an $8 \times 10$ matrix of the form

$$
\frac{\partial\left(x_{1}, v_{1}, x_{4}, v_{4}\right)^{R}}{\partial\left(L_{3}, \ell_{3}, G_{3}, g_{3}, x_{1}, v_{1}, G_{4}, g_{4}\right)^{R}}=\chi u_{i} \otimes l_{i}+\left[\begin{array}{cc|cc}
0_{4 \times 4} & \mathrm{Id}_{4 \times 4} & 0_{4 \times 1} & 0_{4 \times 1} \\
\hline 0_{1 \times 4} & 0_{1 \times 4} & 0 & 0 \\
0_{1 \times 4} & 0_{1 \times 4} & O(1) & O(1) \\
\breve{l_{i}} & & O(1 / \chi) & O(1 / \chi) \\
0_{1 \times 4} & 0_{1 \times 4} & 0 & 0
\end{array}\right]_{8 \times 10},
$$

where we have the estimates

$$
\begin{aligned}
u_{i} & =\left(0_{1 \times 5}, \frac{L_{4}}{2 m_{4}^{2} k_{4}^{2}}, 0, \frac{1}{\chi}\right)_{1 \times 8}^{T}, \\
\breve{l}_{i} & \lesssim\left(1, \frac{1}{\chi^{3}}, \frac{1}{\chi^{3}}, \frac{1}{\chi^{3}} ; \frac{1}{\mu \chi^{2}}, \frac{1}{\chi^{3}}, \mu, \frac{\mu}{\chi}\right)_{1 \times 8}, \\
l_{i} & =\left(\frac{G_{4} k_{4} m_{4}}{L_{4}\left(G_{4}^{2}+L_{4}^{2}\right)}, O\left(\frac{1}{\chi^{3}}\right)_{1 \times 3} ; O\left(\frac{1}{\mu \chi^{2}}, \frac{1}{\chi^{3}}, \mu, \frac{\mu}{\chi}\right) ;-\frac{k_{4} m_{4}}{G_{4}^{2}+L_{4}^{2}},-\frac{k_{4} m_{4}}{L_{4}}\right)_{1 \times 10}^{R}
\end{aligned}
$$

and $l_{i}$ converges to $\hat{\overline{\mathbf{l}}}$ defined in Lemma 3.2 as $1 / \chi \ll \mu \rightarrow 0$.

(b) On the section $\left\{x_{4, \|}^{L}=\frac{1}{2} \chi\right\}$, the matrix $\partial\left(\mathcal{X}_{1}, \mathcal{X}_{4}\right)^{L} / \partial \mathcal{V}^{L}$ for (IV) has the same form with the same $u_{i}$, and $l_{i}$ is replaced by

$$
l_{i^{\prime}}=\left(0_{1 \times 8},-\frac{k_{4} m_{4}}{L_{4}^{2}},-\frac{k_{4} m_{4}}{L_{4}}\right)+O\left(\frac{1}{\chi},\left(\frac{1}{\chi^{4}}\right)_{1 \times 3} ; \frac{1}{\mu \chi^{3}}, \frac{1}{\chi^{4}}, \frac{\mu}{\chi}, \frac{\mu}{\chi^{2}} ; \frac{1}{\chi^{3}}, 0\right)_{1 \times 10} .
$$

Proof. We trivially have

$$
\left(\frac{\partial \mathcal{X}_{1}}{\partial\left(\mathcal{V}_{3}, \mathcal{V}_{4}\right)}\right)^{R, L}=0 \text { and }\left(\frac{\partial \mathcal{X}_{1}}{\partial \mathcal{V}_{1}}\right)^{R, L}=\operatorname{Id}_{4}
$$

since the variables $\mathcal{X}_{1}=\left(x_{1}, v_{1}\right)$ are not transformed to Delaunay variables and they are independent of $\mathcal{V}_{3,4}$. It remains to obtain $\partial \mathcal{X}_{4} / \partial \mathcal{V}$.

Step 1. (Formal derivations.)

In the following calculation, we use (8.8). The formal calculation works for both cases, left and right, so we omit the superscripts. As before, we use $\partial$ to denote the partial derivative with respect to all twelve variables, and $\nabla$ to denote the covariant derivative with respect to the ten variables, with $L_{4}$ and $\ell_{4}$ eliminated. Since we are 
restricted to the section $x_{4, \|}= \pm \frac{1}{2} \chi$, we will solve $\ell_{4}$ as functions of $L_{4}, G_{4}$ and $g_{4}$, and we use $\delta \ell_{4} / \delta L_{4}, \delta \ell_{4} / \delta G_{4}$ and $\delta \ell_{4} / \delta g_{4}$ for the corresponding partial derivatives. We have

$$
\begin{aligned}
& \nabla_{\mathcal{V}} \mathcal{X}_{4} \\
& =\frac{\partial \mathcal{X}_{4}}{\partial\left(L_{4}, \ell_{4}\right)} \nabla_{\mathcal{V}}\left(L_{4}, \ell_{4}\right)+\left(0_{4 \times 8} \mid \frac{\partial \mathcal{X}_{4}}{\partial\left(G_{4}, g_{4}\right)}\right) \\
& =\left(\frac{\partial \mathcal{X}_{4}}{\partial L_{4}}, \frac{\partial \mathcal{X}_{4}}{\partial \ell_{4}}\right)\left(\frac{\delta \ell_{4}}{\delta L_{4}} \nabla_{\mathcal{V}} L_{4}+\left(0_{1 \times 8} ; \frac{\delta \ell_{4}}{\delta G_{4}}, \frac{\delta \ell_{4}}{\delta g_{4}}\right)\right)+\left(0_{4 \times 8} \mid \frac{\partial \mathcal{X}_{4}}{\partial G_{4}}, \frac{\partial \mathcal{X}_{4}}{\partial g_{4}}\right) \\
& =\left(\frac{\partial \mathcal{X}_{4}}{\partial L_{4}}+\frac{\delta \ell_{4}}{\delta L_{4}} \frac{\partial \mathcal{X}_{4}}{\partial \ell_{4}}\right) \otimes \nabla_{\mathcal{V}} L_{4}+\frac{\partial \mathcal{X}_{4}}{\partial \ell_{4}} \otimes\left(0_{1 \times 8} ; \frac{\delta \ell_{4}}{\delta G_{4}}, \frac{\delta \ell_{4}}{\delta g_{4}}\right)+\left(0_{4 \times 8} \mid \frac{\partial \mathcal{X}_{4}}{\partial G_{4}}, \frac{\partial \mathcal{X}_{4}}{\partial g_{4}}\right) \\
& =\left(\frac{\partial \mathcal{X}_{4}}{\partial L_{4}}-\frac{\frac{\partial x_{4, \|}}{\partial L_{4}}}{\frac{\partial x_{4, \|}}{\partial \ell_{4}}} \frac{\partial \mathcal{X}_{4}}{\partial \ell_{4}}\right) \otimes \nabla \mathcal{V} L_{4}-\frac{1}{\frac{\partial x_{4, \|}}{\partial \ell_{4}}} \frac{\partial \mathcal{X}_{4}}{\partial \ell_{4}} \otimes\left(0_{1 \times 8} ; \frac{\partial x_{4, \|}}{\partial G_{4}}, \frac{\partial x_{4, \|}}{\partial g_{4}}\right) \\
& +\left(0_{4 \times 8} \mid \frac{\partial \mathcal{X}_{4}}{\partial G_{4}}, \frac{\partial \mathcal{X}_{4}}{\partial g_{4}}\right)
\end{aligned}
$$

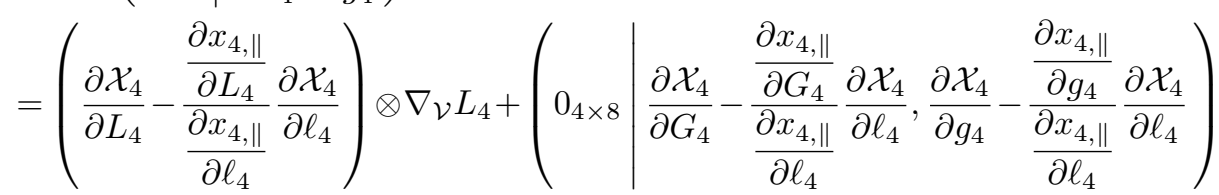

$$
\begin{aligned}
& \sim\left(0,-\frac{G_{4} L_{4}^{2} \ell_{4}}{m_{4} k_{4}\left(G_{4}^{2}+L_{4}^{2}\right)}+O(1), \frac{k_{4} m_{4}}{L_{4}^{2}}, \frac{G_{4} k_{4} m_{4}}{L_{4}\left(G_{4}^{2}+L_{4}^{2}\right)}\right)^{T} \otimes \nabla \mathcal{V} L_{4} \\
& +\left(0_{4 \times 8} \mid \begin{array}{cc}
0 & 0 \\
\frac{L_{4}^{3} \ell_{4} / m_{4} k_{4}}{G_{4}^{2}+L_{4}^{2}}+O(1) & \frac{L_{4}^{2} \ell_{4}}{m_{4} k_{4}}+O(1) \\
\frac{1}{\chi} & \frac{1}{\chi} \\
-\frac{k_{4} m_{4}}{G_{4}^{2}+L_{4}^{2}} & -\frac{k_{4} m_{4}}{L_{4}}
\end{array}\right)_{4 \times 10}
\end{aligned}
$$

where, in the last step, we use Lemma A.3, and choose the sign $\sigma=\operatorname{sign}(u)$ to be "+" for both part (a) and (b) of the lemma. Actually, the terms

$$
\frac{\frac{\partial x_{4, \|}}{\partial\left(L_{4}, G_{4}, g_{4}\right)}}{\frac{\partial x_{4, \|}}{\partial \ell_{4}}} \frac{\partial \mathcal{X}_{4}}{\partial \ell_{4}}
$$

are small compared to the corresponding $\partial \mathcal{X}_{4} / \partial\left(L_{4}, G_{4}, g_{4}\right)$, due to the smallness of $\partial\left(x_{4, \perp}, v_{4}\right) / \partial \ell_{4}$ in (A.7). 
It is easy to see from the above calculation that the first row is zero, since the first entry of $\mathcal{X}_{4}$ is $x_{4, \|}$. This also follows from the fact that we are restricted on the sections $\left\{x_{4, \|}^{R}=-\frac{1}{2} \chi\right\}$ and $\left\{x_{4, \|}^{L}=\frac{1}{2} \chi\right\}$, so that $x_{4, \|}$ is a constant. We already have the tensor structure in the first summand of the last line of (9.3). Next, in the second summand of (9.3), the two non-trivial columns are nearly parallel. The reason is that in equation (A.7) the two vectors

$$
\frac{\partial x_{4, \perp}}{\partial\left(L_{4}, G_{4}, g_{4}\right)} \quad \text { and } \quad \frac{\partial v_{4, \perp}}{\partial\left(L_{4}, G_{4}, g_{4}\right)}
$$

(the second and fourth rows in (A.7)) are parallel with ratio of modulus $L_{4}^{3} \ell_{4} / m_{4}^{2} k_{4}^{2}$, if we discard the $O(1)$ terms in the former (see Remark A.4). The two terms

$$
-\frac{\frac{\partial x_{4, \|}}{\partial G_{4}}}{\frac{\partial x_{4, \|}}{\partial \ell_{4}}} \frac{\partial \mathcal{X}_{4}}{\partial \ell_{4}} \quad \text { and } \quad-\frac{\frac{\partial x_{4, \|}}{\partial g_{4}}}{\frac{\partial x_{4, \|}}{\partial \ell_{4}}} \frac{\partial \mathcal{X}_{4}}{\partial \ell_{4}}
$$

are obviously parallel.

Step 2. (The case $\partial\left(\mathcal{X}_{1}, \mathcal{X}_{4}\right)^{R} / \partial \mathcal{V}^{R}$ on the section $\left\{x_{4, \|}^{R}=-\frac{1}{2} \chi\right\}$.)

Orbit parameters in this step should carry a superscript $R$ which we omit for simplicity. We define the last row of the above calculation (9.3) as the vector $l_{i}$. That is,

$$
l_{i}:=\left(\frac{G_{4} k_{4} m_{4}}{L_{4}\left(G_{4}^{2}+L_{4}^{2}\right)}\right) \cdot \nabla_{\mathcal{V}} L_{4}+\left(0_{1 \times 8} ;-\frac{k_{4} m_{4}}{G_{4}^{2}+L_{4}^{2}},-\frac{k_{4} m_{4}}{L_{4}}\right) .
$$

We get the estimate of $l_{i}$ stated in the lemma using Lemma 7.2 for the section $\left\{x_{4, \|}^{R}=-\frac{1}{2} \chi\right\}$. Moreover, since the first entry in $\nabla_{\mathcal{V}} L_{4}$ is $\nabla_{L_{3}} L_{4}=1+O(\mu)$ and the last two entries are $O\left(1 / \chi^{2}\right)$, we see that $l_{i} \rightarrow \hat{\overline{\mathbf{l}}}$ defined in Lemma 3.2 when we take limit $1 / \chi \ll \mu \rightarrow 0$.

Then, the second row of the last line of (9.3) is

$$
\left(-\frac{L_{4}^{3} \ell_{4}}{m_{4}^{2} k_{4}^{2}}+O(1)\right) l_{i}+\left(0_{1 \times 8} ; O(1), O(1)\right) .
$$

For the third row, we define a vector

$$
\breve{l}_{i}=\left(1, \frac{1}{\chi^{3}}, \frac{1}{\chi^{3}}, \frac{1}{\chi^{3}} ; \frac{1}{\mu \chi^{2}}, \frac{1}{\chi^{3}}, \mu, \frac{\mu}{\chi}\right)_{1 \times 8},
$$

as the first eight entries of $l_{i}$, so the third row is $\left(\breve{l}_{i} ; 1 / \chi, 1 / \chi\right)_{1 \times 10}$. 
Finally, we define

$$
u_{i}=\left(0,0,0,0 ; 0, \frac{L_{4}}{2 m_{4}^{2} k_{4}^{2}}, 0, \frac{1}{\chi}\right)_{1 \times 8}^{T},
$$

which, after removing the first four zeros, is almost parallel to the last two columns in the last row of (9.3), where we used (A.5) to get that $L_{4}^{2} \ell_{4} \simeq-\frac{1}{2} \chi$, when restricted to the section $\left\{x_{4, \|}^{R}=-\frac{1}{2} \chi\right\}$. This completes the proof of part (a).

Step 3. (The case of $\partial\left(\mathcal{X}_{1}, \mathcal{X}_{4}\right)^{L} / \partial \mathcal{V}^{L}$ on the section $\left\{x_{4, \|}^{L}=\frac{1}{2} \chi\right\}$.)

This case follows from the same formal calculation (9.3). However, since the variables are to the left of the section $x_{4, \|}^{L}=\frac{1}{2} \chi$, we have $G_{4}^{L}=O(1 / \chi)$ in (9.4) according to Lemma $6.11(\mathrm{~b})$. Thus, we get an improved $l_{i^{\prime}}$ in place of $l_{i}$, by applying Lemma 7.2 to (9.4). We also have $L_{4}^{2}\left(-\ell_{4}\right) \simeq \frac{1}{2} \chi$ using (A.6) for $u>0$ and $\ell_{4}<0$ to the left of the section $x_{4, \|}^{L}=\frac{1}{2} \chi$. So, $u_{i}$ in the left case has the same expression as in the right case. This proves part (b).

\subsection{From Cartesian to Delaunay coordinates}

In this section we compute

$$
\frac{\partial\left(\mathcal{V}_{1} ; \mathcal{V}_{4}\right)}{\partial\left(\mathcal{X}_{1}, \mathcal{X}_{4}\right)}=\frac{\partial\left(x_{1}, v_{1}, G_{4}, g_{4}\right)}{\partial\left(x_{1}, v_{1}, x_{4}, v_{4}\right)}
$$

The key observation to get the tensor structure is explained in Remark A.4 (3).

Sublemma 9.2. Assume AG. Then, the following holds.

(a) On the section $\left\{x_{4, \|}^{R}=-\frac{1}{2} \chi\right\}$, the matrix $\partial\left(\mathcal{V}_{1} ; \mathcal{V}_{4}\right)^{L} / \partial\left(\mathcal{X}_{1}, \mathcal{X}_{4}\right)^{L}$ in (9.1) is a $6 \times 8$ matrix of the following form:

$$
\frac{\partial\left(x_{1}, v_{1}, G_{4}, g_{4}\right)^{L}}{\partial\left(x_{1}, v_{1}, x_{4}, v_{4}\right)^{L}}=\chi u_{i i i} \otimes l_{i i i}+\left[\begin{array}{c|cccc}
\mathrm{Id}_{4 \times 4} & 0_{4 \times 1} & 0_{4 \times 1} & 0_{4 \times 1} & 0_{4 \times 1} \\
\hline 0_{1 \times 4} & 0 & 0 & 0 & 0 \\
0_{1 \times 4} & O\left(1 / \chi^{2}\right) & O\left(1 / \chi^{2}\right) & O(1) & O(1)
\end{array}\right]_{6 \times 8},
$$

where we have estimates

$$
\begin{aligned}
u_{i i i} & =\left(0,0,0,0 ; 1, \frac{1}{L_{4}}+O\left(\frac{1}{\chi^{2}}\right)\right)_{1 \times 6}^{T}, \\
l_{i i i} & =\left(0_{1 \times 4} ; O\left(\frac{1}{\chi^{2}}\right), \frac{-m_{4} k_{4}}{\chi L_{4}}, O\left(\frac{1}{\chi}\right),-\frac{1}{2}\right)_{1 \times 8} .
\end{aligned}
$$

(b) On the section $\left\{x_{4, \|}^{L}=\frac{1}{2} \chi\right\}$ the matrix $\partial\left(\mathcal{V}_{1} ; \mathcal{V}_{4}\right)^{R} / \partial\left(\mathcal{X}_{1}, \mathcal{X}_{4}\right)^{R}$ for (IV) has the same form with $u_{i i i}$ replaced by

$$
u_{i i i^{\prime}}=\left(0,0,0,0 ; 1, \frac{L_{4}}{G_{4}^{2}+L_{4}^{2}}\right)_{1 \times 6}^{T},
$$

and $l_{i i i}$ replaced by $l_{i i i^{\prime}}=-l_{i i i}$. 
Proof. The only non-trivial part of this matrix is $\partial\left(G_{4}, g_{4}\right) / \partial\left(x_{4}, v_{4}\right)$. We consider first part (a), to the left of the section $\left\{x_{4, \|}^{R}=-\frac{1}{2} \chi\right\}$, where the variables should carry superscript $L$ that we omit. It follows from Lemma A.5 (b) that

$$
\frac{\partial g_{4}}{\partial\left(x_{4}, v_{4}\right)}=\frac{L_{4}}{G_{4}^{2}+L_{4}^{2}} \frac{\partial G_{4}}{\partial\left(x_{4}, v_{4}\right)}+O\left(\frac{1}{\chi^{2}}, \frac{1}{\chi^{2}}, 1,1\right) .
$$

This implies that the two rows in $\partial\left(G_{4}, g_{4}\right)^{L} / \partial\left(x_{4}, v_{4}\right)^{L}$ are almost parallel up to the $O$ term. Therefore, we have the tensor structure in the lemma.

Next, we define

$$
\begin{aligned}
u_{i i i} & =\left(0_{1 \times 4} ; 1, \frac{L_{4}}{G_{4}^{2}+L_{4}^{2}}\right)^{T}=\left(0_{1 \times 4} ; 1, \frac{1}{L_{4}}+O\left(\frac{1}{\chi^{2}}\right)\right)^{T}, \\
l_{i i i} & =\frac{1}{\chi}\left(0_{1 \times 4} ; \frac{\partial G_{4}}{\partial\left(x_{4}, v_{4}\right)}\right)=\left(0_{1 \times 4} ; O\left(\frac{1}{\chi^{2}}\right),-\frac{m_{4} k_{4}}{\chi L_{4}}, O\left(\frac{1}{\chi}\right),-\frac{1}{2}\right),
\end{aligned}
$$

where the entry $-m_{4} k_{4} / \chi L_{4}$ is obtained using the following formulas:

$$
\frac{\partial G}{\partial Q_{\perp}}=P_{\|}\left(\text {by Lemma A.5), } \quad E_{4}=\frac{|P|^{2}}{2 m_{4}}-\frac{k_{4}}{|Q|}=\frac{m_{4} k_{4}^{2}}{2 L_{4}^{2}}, \quad|P| \simeq\left|P_{\|}\right| \quad \text { and } \quad P_{\|}<0 .\right.
$$

This gives the matrix stated in the sublemma. In part (a), all the Cartesian and Delaunay variables are immediately to the left of the section $\left\{x_{4, \|}^{R}=-\frac{1}{2} \chi\right\}$, so we have $G_{4}^{L}=O(1 / \chi)$, using Lemma 6.11 and $x_{4, \|}^{L} \simeq \frac{1}{2} \chi$. Next, we consider part (b). It follows from Lemma A.5 that, to the right of the section $\left\{x_{4, \|}^{L}=\frac{1}{2} \chi\right\}$ the matrix $\partial\left(\mathcal{V}_{1} ; \mathcal{V}_{4}\right)^{R} / \partial\left(\mathcal{X}_{1}, \mathcal{X}_{4}\right)^{R}$ has the same estimates as in the left case with

$$
\begin{aligned}
u_{i i i^{\prime}} & =\left(0_{1 \times 4} ; 1, \frac{L_{4}}{G_{4}^{2}+L_{4}^{2}}\right)^{T}, \\
l_{i i i^{\prime}} & =\frac{1}{\chi}\left(0_{1 \times 4} ; \frac{\partial G_{4}}{\partial\left(x_{4}, v_{4}\right)}\right)=\left(0_{1 \times 4} ; O\left(\frac{1}{\chi^{2}}\right), \frac{m_{4} k_{4}}{\chi L_{4}}, O\left(\frac{1}{\chi}\right), \frac{1}{2}\right) .
\end{aligned}
$$

We see that $l_{i i i^{\prime}}$ gets a "minus" sign compared to $l_{i i i}$, since both $P_{\|}$and $Q_{\|}$get "minus" signs.

With the two sublemmas, we can complete the computation of the matrices (II) and (IV).

Computation of matrices (II) and (IV) in Proposition 5.2. To be compatible with the formal derivation in (9.1), we add four zeros to $u_{i}$ as the new first four entries. We still denote the new vector of twelve components by $u_{i}$, as stated in Proposition 5.2. We also define a $12 \times 10$ matrix

$$
C=\left[\begin{array}{cc}
\operatorname{Id}_{4 \times 4} & 0_{4 \times 6} \\
& *
\end{array}\right]
$$


where $*$ is the $O(1)$ matrix in Sublemma 9.1.

Then, consider Sublemma 9.2. To be compatible with the formal derivation in (9.1), we enlarge $u_{i i i}$ and $u_{i i i^{\prime}}$ by adding four zeros as the new first four entries to get vectors in $\mathbb{R}^{10}$. We define a $10 \times 12$ matrix

$$
A=\left[\begin{array}{cc}
\operatorname{Id}_{4 \times 4} & 0_{4 \times 8} \\
0_{6 \times 4} & *
\end{array}\right]
$$

where $*$ is the $O(1)$ matrix of Sublemma 9.2.

Fitting these manipulations into (9.1) gives

$$
\begin{aligned}
(\mathrm{II}) & =\left(\chi u_{i i i} \otimes l_{i i i}+A\right) R \cdot L^{-1}\left(\chi u_{i} \otimes l_{i}+C\right), \\
(\mathrm{IV}) & =\left(\chi u_{i i i^{\prime}} \otimes l_{i i i^{\prime}}+A\right) L \cdot R^{-1}\left(\chi u_{i} \otimes l_{i^{\prime}}+C\right) .
\end{aligned}
$$

\section{The local map}

The section $\left\{\left|q_{3}-q_{4}\right|=\mu^{\kappa}\right\} \quad\left(\frac{1}{3}<\kappa<\frac{1}{2}\right)$ cuts the orbit for the local map into three pieces:

$$
\begin{aligned}
\left\{x_{4, \|}^{R}=-2\right\} & \longrightarrow\left\{\left|q_{3}-q_{4}\right|=\mu^{\kappa}\right\}, \\
\left\{\left|q_{3}-q_{4}\right|=\mu^{\kappa}\right\} & \longrightarrow\left\{\left|q_{3}-q_{4}\right|=\mu^{\kappa}\right\}, \\
\left\{\left|q_{3}-q_{4}\right|=\mu^{\kappa}\right\} & \longrightarrow\left\{x_{4, \|}^{R}=-2\right\} .
\end{aligned}
$$

We define three maps $\mathbb{L}^{-}, \mathbb{L}^{0}$ and $\mathbb{L}^{+}$corresponding to the three pieces, respectively, and we have

$$
\mathbb{L}=\mathbb{L}^{+} \circ \mathbb{L}^{0} \circ \mathbb{L}^{-}
$$

Notation 10.1. - We use the superscript $+($ or - ) to denote the value of the orbit parameters exiting (or entering) the circle $\left|q_{3}-q_{4}\right|=\mu^{\kappa}$.

- Also recall the coordinates $q_{-}$and $p_{-}$for the relative motion, and $q_{+}$and $p_{+}$for the motion of the center of mass of $Q_{3}$ and $Q_{4}$ in (4.10), i.e. $q_{ \pm}=\frac{1}{2}\left(q_{3} \pm q_{4}\right)$ and $p_{ \pm}=p_{3} \pm p_{4}$.

- We introduce the notation $\mathbf{q}=\left(q_{+}, q_{1}\right)$ and $\mathbf{p}=\left(p_{+}, p_{1}\right)$ to handle the center of mass and the remote body simultaneously.

The Hamiltonian for $\mathbb{L}^{ \pm}$is the following given in Lemma 4.7:

$$
H=\left(\frac{v_{1}^{2}}{2 m_{1 R}}-\frac{k_{1 R}}{\left|x_{1}\right|}\right)-\frac{m_{3 R} k_{3 R}^{2}}{2 L_{3}^{2}}+\frac{m_{4 R} k_{4 R}^{2}}{2 L_{4}^{2}}-\frac{\mu}{\left|x_{3} /(1+\mu)-x_{4}\right|}+V_{\text {out }},
$$

where

$$
V_{\text {out }}\left(x_{3}, x_{1}, x_{4}\right)=O\left(\mu+\frac{\mu}{\chi^{2}}+\frac{1}{\chi^{3}}\right),
$$


and the coordinates are Delaunay coordinates from (2.3), and the Hamiltonian for $\mathbb{L}^{0}$ is given in Lemma 4.8:

$$
H\left(q_{1}, p_{1} ; q_{-}, p_{-} ; q_{+}, p_{+}\right)=\left(\mu p_{1}^{2}-\frac{1+2 \mu}{\mu\left|q_{1}\right|}\right)+\left(\frac{1+2 \mu}{4} p_{+}^{2}-\frac{2}{\left|q_{+}\right|}\right)+\left(\frac{1}{4} p_{-}^{2}-\frac{\mu}{2\left|q_{-}\right|}\right)+V_{\mathrm{in}},
$$

where

$$
V_{\mathrm{in}}\left(q_{1}, p_{1} ; q_{-} ; q_{+}, p_{+}\right)=\mu\left\langle p_{1}, p_{+}\right\rangle-\frac{3\left\langle q_{+}, q_{-}\right\rangle^{2}}{2\left|q_{+}\right|^{5}}+\frac{\left|q_{-}\right|^{2}}{\left|q_{+}\right|^{3}}-\frac{\left\langle q_{1}, q_{+}\right\rangle}{\left|q_{1}\right|^{3}}+O\left(\left|q_{-}\right|^{3}+\frac{1}{\left|q_{1}\right|^{3}}\right),
$$

and the coordinates are the relative motion and center of mass coordinates (4.10).

\section{1. $\mathscr{C}^{0}$ control of the local map: proof of Lemma 2.10}

In this section, we obtain the $\mathscr{C}^{0}$ estimate of the local map, based on which we prove Lemma 2.10 .

Suppose the assumption AL for the local map is satisfied. Then, the orbit parameters

$$
\boldsymbol{x}^{ \pm}=\left(x_{1}, v_{1} ; L_{3}, \ell_{3}, G_{3}, g_{3} ; L_{4}, \ell_{4}, G_{4}, g_{4}\right)^{R, \pm}
$$

evaluated on the section $\left\{x_{4, \|}^{R}=-2, \pm v_{4, \|}^{R}<0\right\}$, satisfy $\left|x_{1}\right| \geqslant \chi$ and all the other variables are $O(1)$, as $1 / \chi \ll \mu \rightarrow 0$.

First, it is clear that the time interval defining the maps $\mathbb{L}^{ \pm}$is $O(1)$. After integrating the Hamiltonian equations obtained from the Hamiltonian (10.1), we see that $V_{\text {out }}$ gives only a perturbation of order $O\left(\mu+\mu / \chi^{2}+1 / \chi^{3}\right)$ to the Kepler motion. Next, the contribution of the term

$$
-\frac{\mu}{\left|x_{3} /(1+\mu)-x_{4}\right|}
$$

to the Hamiltonian equation is estimated as

$$
\frac{\mu}{\left|x_{3} /(1+\mu)-x_{4}\right|^{2}}
$$

and, after integration its perturbation to the Kepler motion is estimated as

$$
\int_{-2}^{\mu^{\kappa}} \frac{\mu}{|t|^{2}} d t=O\left(\mu^{1-\kappa}\right)
$$

since relative velocity is non-zero, and the orbit approaches close encounter nearly linearly in $t$. So the orbit parameters are Kepler motions with $o(1)$ perturbations as $1 / \chi \ll \mu \rightarrow 0$. In particular, except for the two variables $\ell_{3}$ and $\ell_{4}$, all the other orbit parameters undergo only a $O\left(\mu^{1-\kappa}\right)\left(\ll \mu^{\kappa}\right)$ perturbation.

Next, we study the dynamics inside $\left\{\left|q_{3}-q_{4}\right|=\mu^{\kappa}\right\}$. The next lemma shows that the map $\mathbb{L}^{0}$ is close to elastic collision. 
LEMma 10.2. Suppose the initial orbit parameters on the section $\left\{x_{4, \|}^{R}=-2, v_{4, \|}^{R}<0\right\}$ satisfy AL. Then, the following holds.

(a) We have the following equations for orbits crossing the section $\left\{\left|q_{3}-q_{4}\right|=\mu^{\kappa}\right\}$, $\frac{1}{3}<\kappa<\frac{1}{2}$, as $\mu \rightarrow 0$ :

$$
\left\{\begin{array}{l}
p_{3}^{+}=\frac{1}{2} \operatorname{Rot}(\alpha)\left(p_{3}^{-}-p_{4}^{-}\right)+\frac{1}{2}\left(p_{3}^{-}+p_{4}^{-}\right)+O\left(\mu^{2(1-\kappa)}+\mu^{3 \kappa-1}\right), \\
p_{4}^{+}=-\frac{1}{2} \operatorname{Rot}(\alpha)\left(p_{3}^{-}-p_{4}^{-}\right)+\frac{1}{2}\left(p_{3}^{-}+p_{4}^{-}\right)+O\left(\mu^{2(1-\kappa)}+\mu^{3 \kappa-1}\right), \\
\left(\mathbf{q}^{+}, \mathbf{p}^{+}\right)=\left(\mathbf{q}^{-}, \mathbf{p}^{-}\right)+O\left(\mu^{k}\right) \\
\left|q_{3}^{-}-q_{4}^{-}\right|=\mu^{\kappa} \\
\left|q_{3}^{+}-q_{4}^{+}\right|=\mu^{\kappa}
\end{array}\right.
$$

where

$$
\operatorname{Rot}(\alpha)=\left[\begin{array}{cc}
\cos \alpha & -\sin \alpha \\
\sin \alpha & \cos \alpha
\end{array}\right]
$$

and

$$
\alpha=\pi+2 \arctan \left(\frac{G_{\text {in }}}{\mu \mathcal{L}_{\text {in }}}\right), \quad \text { where } \frac{1}{4 \mathcal{L}_{\text {in }}^{2}}=\frac{p_{-}^{2}}{4}-\frac{\mu}{2\left|q_{-}\right|} \text {and } G_{\text {in }}=2 p_{-} \times q_{-} .
$$

(b) We have $1 / c<\mathcal{L}_{\mathrm{in}}<c$ for some constant $c>1$. If $\alpha$ is bounded away from zero and $\pi$ by an angle independent of $\mu$, then $G_{\mathrm{in}}=O(\mu)$, and the closest distance between $q_{3}$ and $q_{4}$ is bounded away from zero by $\mu / c$ and from above by $\mu c$.

(c) If $\alpha$ is bounded away from zero and $\pi$ by an angle independent of $\mu$, then, when measured on the boundary of the circle $\left|q_{-}\right|=2 \mu^{\kappa}$, the angle between $q_{-}$and $p_{-}$is

$$
O\left(\mu^{1-\kappa}\right)
$$

(d) The time interval during which the orbit stays in the circle $\left|q_{3}-q_{4}\right|=\mu^{\kappa}$ is

$$
\Delta t=O\left(\mu^{\kappa}\right)
$$

Proof. From (10.2), we decompose the Hamiltonian as $H=H_{\text {rel }}+\mathfrak{h}(\mathbf{q}, \mathbf{p})$, where the Hamiltonian $H_{\text {rel }}$ governs the relative motion consisting of all the terms containing $q_{-}$ or $p_{-}$in (10.2), as

$$
H_{\mathrm{rel}}=\frac{\mu^{2}}{4 L_{-}^{2}}+\frac{\left|q_{-}\right|^{2}}{\left|q_{+}\right|^{3}}-\frac{3\left\langle q_{+}, q_{-}\right\rangle^{2}}{2\left|q_{+}\right|^{5}}+O\left(\mu^{3 \kappa}+\frac{1}{\chi^{2}}\right),
$$

where

$$
\frac{\mu^{2}}{4 L_{-}^{2}}=\frac{1}{4} p_{-}^{2}-\frac{\mu}{2\left|q_{-}\right|}
$$


is in Delaunay coordinates and $L_{-}=\mu \mathcal{L}_{\text {in }}$.

Fix a small number $\delta_{1}$. Below we derive several estimates valid for the first $\delta_{1}$ units of time the orbit spends in the set $\left|q_{-}\right| \leqslant \frac{1}{2} \mu^{k}$. We then show that $\Delta t \ll \delta_{1}$. It will be convenient to measure time from when the orbit enters the set $\left|q_{-}\right|<\frac{1}{2} \mu^{k}$.

Note that $H$ is preserved and $\dot{\mathfrak{h}}=O(1)$, which implies that $L / \mu$ is $O(1)$, and moreover that this ratio does not change much for $t \in\left[0, \delta_{1}\right]$. Using the identity

$$
\frac{\mu^{2}}{4 L^{2}}=\frac{p_{-}^{2}}{4}-\frac{\mu}{2\left|q_{-}\right|}
$$

we see that initially $L / \mu$ is uniformly bounded from below for the orbits by Lemma 2.10. Thus, there is a constant $\delta_{2}$ such that, for $t \in\left[0, \delta_{1}\right]$, we have

$$
\delta_{2} \mu \leqslant L(t) \leqslant \frac{\mu}{\delta_{2}}
$$

From (6.6), we obtain

$$
O\left(\mu^{\kappa}\right)=\left|q_{-}\right|=\frac{L^{2}}{\mu}(e \cosh u-1)
$$

with $\ell$ and $u$ related by $u-e \sinh u=\ell$. This gives

$$
\ell=O\left(\mu^{\kappa-1}\right)
$$

Next,

$$
\dot{\ell}=\frac{\partial H}{\partial L}=-\frac{\mu^{2}}{2 L^{3}}-\frac{\partial H_{\mathrm{rel}}}{\partial q_{-}} \frac{\partial q_{-}}{\partial L}=-\frac{\mu^{2}}{2 L^{3}}+O\left(\mu^{\kappa}\right) O\left(\mu^{\kappa-1}\right)=-\frac{\mu^{2}}{2 L^{3}}+O\left(\mu^{2 \kappa-1}\right) .
$$

Since the leading term here is at least $\delta_{2}^{3} / 2 \mu$, while $\ell=O\left(\mu^{\kappa-1}\right)$, we obtain part (d) of the lemma. In particular, the estimates derived above are valid for the time the orbit spends in $\left|q_{-}\right| \leqslant \frac{1}{2} \mu^{\kappa}$. Next, without using any control on $G$, using the inequality

$$
\left|\frac{\partial e}{\partial G}\right|=\frac{1}{L} \frac{G / L}{e} \leqslant \frac{1}{L}
$$

we have

$$
\begin{aligned}
\dot{L} & =-\frac{\partial H}{\partial q_{-}} \frac{\partial q_{-}}{\partial \ell}=O\left(\mu^{\kappa+1}\right), \\
\dot{G} & =-\frac{\partial H}{\partial q_{-}} \frac{\partial q_{-}}{\partial g}=O\left(\left|q_{-}\right|^{2}\right)=O\left(\mu^{2 \kappa}\right), \\
\dot{g} & =\frac{\partial H}{\partial q_{-}} \frac{\partial q_{-}}{\partial G}=O\left(\mu^{\kappa}\right) O\left(\mu^{\kappa-1}\right)=O\left(\mu^{2 \kappa-1}\right) .
\end{aligned}
$$


Integrating over time $\Delta t=O\left(\mu^{\kappa}\right)$, we get that the oscillation of $g$ and $\arctan (G / L)$ are $O\left(\mu^{3 \kappa-1}\right)$. We are now ready to derive the first two equations of (10.3). It is enough to show that

$$
p_{-}^{+}=R(\alpha) p_{-}^{-}+O\left(\mu^{2(1-\kappa)}+\mu^{3 \kappa-1}\right),
$$

where $\alpha=2 \arctan (G / L)$ is the angle formed by the two asymptotes of the Kepler hyperbolic motion. We first have $\left|p_{-}^{+}\right|=\left|p_{-}^{-}\right|+O\left(\mu^{\kappa}\right)$, using the total energy conservation. It remains to show the expression of $\alpha$. Let us denote $\phi=\arctan (G / L)$ and

$$
p_{-}=\left(p_{1}, p_{2}\right), \quad\left(p_{1}, p_{2}\right)=R(g)\left(-\frac{\mu}{L} \frac{\sinh u}{1-e \cosh u},-\frac{\mu G}{L^{2}} \frac{\cosh u}{1-e \cosh u}\right),
$$

using (A.3). We have by (10.7) that $e^{|u|} \sim \ell \sim \mu^{\kappa-1}$. Thus,

$$
\begin{aligned}
\frac{p_{2}}{p_{1}} & =\frac{-\frac{\mu G}{L^{2}} \cosh u \cos g-\frac{\mu}{L} \sinh u \sin g}{\frac{\mu G}{L^{2}} \cosh u \sin g-\frac{\mu}{L} \sinh u \cos g}=\frac{\frac{G}{L} \pm \tan g}{ \pm 1-\frac{G}{L} \tan g}+e^{-2|u|} E\left(\frac{G}{L}, g, u\right) \\
& =\tan (g \pm \phi)+O\left(\mu^{2(1-\kappa)}\right),
\end{aligned}
$$

where the $\operatorname{sign} \pm$ is taken as $\operatorname{sign}(u)$ and $E$ is a $O(1)$ function as $|u| \rightarrow \infty$. Since arctan is globally Lipschitz, this completes the proof of part (a) by choosing $\alpha=2 \phi$.

From the Hamiltonian equations for $\dot{\mathbf{q}}$, we obtain

$$
\mathbf{q}^{+}=\mathbf{q}^{-}+O\left(\mu^{\kappa}\right)
$$

We also have $q_{-}^{+}=q_{-}^{-}+O\left(\mu^{\kappa}\right)$, due to to the definition of the sections $\left\{\left|q_{-}^{ \pm}\right|=\frac{1}{2} \mu^{\kappa}\right\}$. This proves the last two equation in (10.3). Plugging (10.12) into the Hamiltonian equation for $\dot{\mathbf{p}}$, we see that $\mathbf{p}^{+}=\mathbf{p}^{-}+O\left(\mu^{\kappa}\right)$. This completes the proof of part (a).

The first claim of part (b) has already been established. The estimate of $G$ follows from the formula for $\alpha$. The estimate of the closest distance follows from the fact that, if $\alpha$ is bounded away from zero and $\pi$, then the $q_{-}(t)$ orbit is a small perturbation of Kepler motion, and for Kepler motion the closest distance is of order $G$. We integrate the $\dot{G}$ equation (10.9) over time $O\left(\mu^{\kappa}\right)$ to get that the total variation $\Delta G$ is at most $\mu^{3 \kappa}$, which is much smaller than $\mu$. So, $G$ is bounded away from zero by a quantity of order $O(\mu)$.

Finally, we get part (c) from the fact that

$$
G=\mu^{\kappa}\left|v_{-}\right| \sin \measuredangle\left(p_{-}, q_{-}\right)=O(\mu) .
$$

We are now ready to prove Lemma 2.10. 
Proof of Lemma 2.10. Since we assume that the outgoing asymptote $\bar{\theta}^{+}$is close to $\pi$, we get that the orbit under consideration has to intersect the section $\left|q_{3}-q_{4}\right|=\mu^{\kappa}$, and also achieve $\left|q_{3}-q_{4}\right|=O(\mu)$ by Lemma $10.2(\mathrm{~b})$. Indeed, it is enough to show that $p_{-}^{+} \times p_{-}^{-}$is bounded away from zero for both collisions. For the first collision,

$$
4\left(1+\varepsilon_{0} \varepsilon_{1}\right)\left(p_{-}^{-} \times p_{-}^{+}\right)=8 \sqrt{5+2 \varepsilon_{0} \varepsilon_{1}}-3\left(\varepsilon_{1}-\varepsilon_{0}\right)>8 \sqrt{5}-3 .
$$

For the second collision,

$$
\varepsilon_{0}^{2}\left(p_{-}^{-} \times p_{-}^{+}\right)=2\left(\varepsilon_{0}+\varepsilon_{1}\right)(1+\sqrt{2})>2(1+\sqrt{2}) .
$$

With the same initial $E_{3}, e_{3}, g_{3}$ and $e_{4}$, we determine a solution of the Gerver map. We have shown at the beginning of the section that the orbit parameters $\left(E_{3}, e_{3}, g_{3}, e_{4}\right)$ have an oscillation of order $O\left(\mu^{1-\kappa}\right)$, and the $\ell_{3}$ variable is solved from the implicit function $\left|q_{3}-q_{4}\right|=\mu^{\kappa}$. We get that the $p_{3,4}^{-}$and $q_{3,4}^{-}$at collision in Gerver's case is close to those values measured on the section $\left|q_{3}-q_{4}\right|=\mu^{\kappa}$ in the $\mu>0$ case. Here, we note that the coordinate change between Cartesian and Delaunay outside the section $\left|q_{3}-q_{4}\right|=\mu^{\kappa}$ is not singular. Letting $\mu=0$ in the first two equations of (10.3), we obtain the equations of elastic collisions. Namely, both the kinetic energy and momentum conservation hold:

$$
\left|p_{3}^{+}\right|^{2}+\left|p_{4}^{+}\right|^{2}=\left|p_{3}^{-}\right|^{2}+\left|p_{4}^{-}\right|^{2} \quad \text { and } \quad p_{3}^{+}+p_{4}^{+}=p_{3}^{-}+p_{4}^{-} \text {. }
$$

On the other hand, the Gerver map G in Lemma 2.10 is also defined through elastic collisions. If we could show that the rotation angle $\alpha$ in the $\mu>0$ case is close to Gerver's case, we then could show that the outgoing information $p_{3,4}^{+}$and $q_{3,4}^{+}$are close in both cases. We then complete the proof using the fact that the orbit outside $\left|q_{3}-q_{4}\right|=\mu^{\kappa}$ is an $O\left(\mu^{1-\kappa}\right)$ small perturbation of the Kepler motion after running the orbit up to the section $\left\{x_{4}=-2\right\}$. By converting $p_{4}^{+}$and $q_{4}^{+}$into Delaunay coordinates, we can express the outgoing asymptote $\bar{\theta}^{+}$as a function of $p_{4}^{+}$and $q_{4}^{+}$, therefore a function of $\alpha, p_{3,4}^{-}$and $q_{3,4}^{-}$using (10.3), where $\mu=0$ corresponds to Gerver's case. To compare the angle $\alpha$, it is enough to show that the outgoing asymptote $\bar{\theta}^{+}$as a function of $\alpha$ has non-degenerate derivative, so that we can apply the implicit function theorem to solve $\alpha$ as a function of $\bar{\theta}^{+}$and the initial conditions. In fact, we have $d \theta^{+}=c \overline{\mathbf{l}}(c \neq 0)$, and from Corollary 10.4 we have $\partial / \partial \alpha=\mathbf{u}$, and hence $d \bar{\theta}^{+} / d \alpha=c \overline{\mathbf{l}} \cdot \mathbf{u}$, which is non-vanishing, due to Lemma 3.4. Here, the vectors $\overline{\mathbf{l}}$ and $\mathbf{u}$ are in Lemmas 3.1 and 3.2 with subscripts omitted. So, the assumption $\left|\bar{\theta}^{+}-\pi\right| \leqslant \tilde{\theta}$ implies that $\alpha$ in (10.3) is $\tilde{\theta}$-close to its value in Gerver's case.

\section{2. $\mathscr{C}^{1}$ control of the local map: proof of Lemma 3.1}

To study the $\mathscr{C}^{1}$ estimate of the local map, we first show that $\mathbb{L}^{+}$and $\mathbb{L}^{-}$are negligible and we then focus on $\mathbb{L}^{0}$. 
Lemma 10.3. Consider the maps $\mathbb{L}^{ \pm}$under the assumption of Lemma 3.1. Then, the vectors $\overline{\mathbf{l}}_{j}$ and $\overline{\mathbf{1}}_{j}, j=1,2$, are almost left-invariant by $d \mathbb{L}^{+}$and $\operatorname{span}\left\{\overline{\mathbf{u}}_{3-j}, \overline{\overline{\mathbf{u}}}_{3-j}\right\}$ is almost right-invariant by $d \mathbb{L}^{-}$in the following sense: as $1 / \chi \ll \mu \rightarrow 0$,

$$
\begin{aligned}
& d \mathbb{L}_{2}^{-} \cdot \operatorname{span}\left\{\overline{\mathbf{u}}_{1}, \overline{\overline{\mathbf{u}}}_{1}\right\}=\operatorname{span}\left\{\overline{\mathbf{u}}_{1}, \overline{\overline{\mathbf{u}}}_{1}\right\}+o(1), \\
& d \mathbb{L}_{1}^{-} d \mathcal{R} \cdot \operatorname{span}\left\{\overline{\mathbf{u}}_{2}, \overline{\mathbf{u}}_{2}\right\}=d \mathcal{R} \cdot \operatorname{span}\left\{\overline{\mathbf{u}}_{2}, \overline{\mathbf{u}}_{2}\right\}+o(1), \\
& \overline{\mathbf{l}}_{j} \cdot d \mathbb{L}_{j}^{+}=\overline{\mathbf{l}}_{j}+o(1), \overline{\overline{\mathbf{l}}}_{j} \cdot d \mathbb{L}_{j}^{+}=\overline{\overline{\mathbf{l}}}_{j}+o(1), \\
& d \widetilde{\mathbb{G}} \cdot \operatorname{span}\left\{\overline{\mathbf{u}}_{2}, \overline{\mathbf{u}}_{2}\right\}=\operatorname{span}\left\{\overline{\mathbf{u}}_{2}, \overline{\mathbf{u}}_{2}\right\}+o(1) .
\end{aligned}
$$

Proof. The proof is again to use (7.1) to reduce the proof into two boundary terms and the fundamental solution of the variational equation. We use the Hamiltonian (10.1). The potential

$$
-\frac{\mu}{\left|\frac{x_{3}}{1+\mu}-x_{4}\right|}
$$

contributes a term of order

$$
-\frac{\mu}{\left|\frac{x_{3}}{1+\mu}-x_{4}\right|^{3}}
$$

to the variational equation and its contribution to the fundamental solution is estimated as the integral

$$
\int_{-2}^{\mu^{\kappa}} \frac{\mu}{|t|^{3}} d t=O\left(\mu^{1-2 \kappa}\right)
$$

since the relative velocity is of order 1 approaching close encounter. Moreover, the terms of $V_{\text {out }}$, all of which are also in $U^{R}$, contribute only $O(\mu)$ to the fundamental solution. (If we let $\chi$ go to infinity and set $\ell_{4}=O(1)$, then all the entries in the matrix in the statement of Lemma 7.3 (a) become $\mu, \mu^{2}$ or zero.) So, the fundamental solution is estimated as $\operatorname{Id}+c e_{2,1}+O\left(\mu^{1-2 \kappa}\right)$, where $e_{2,1}$ is the matrix whose $(2,1)$ entry is 1 and 0 otherwise, and $c$ is a constant. For the boundary terms in (7.1), the estimate $\mathcal{F}$ of the Hamiltonian equations is $\left(0,-1,0_{1 \times 8}\right)+O\left(\mu^{1-2 \kappa}\right)$, given in $\S 10.1$ for $\left\{\left|q_{3}-q_{4}\right|=\mu^{\kappa}\right\}$ as well as Corollary 6.10 for the section $\left\{x_{4, \|}^{R}=-2\right\}$. It remains to estimate the term $\nabla_{\mathcal{V}} \ell_{4}$ in (7.1). This estimate on the section $\left\{x_{4, \|}^{R}=-2\right\}$ is given in $\S 8.1$ and $\S 8.3$ as equation (8.3). We next show the estimate of $\nabla \mathcal{V} \ell_{4}$ on the section $\left\{\left|q_{3}-q_{4}\right|=\mu^{\kappa}\right\}$. We have

$$
\nabla_{\mathcal{V} \ell_{4}}=-\left(\frac{\partial\left|q_{3}-q_{4}\right|}{\partial \ell_{4}}\right)^{-1} \nabla_{\mathcal{V}}\left|q_{3}-q_{4}\right|=-\frac{\left(q_{3}-q_{4}\right) \cdot \nabla_{\mathcal{V}}\left(q_{3}-q_{4}\right)}{\left(q_{3}-q_{4}\right) \cdot \frac{\partial\left(q_{3}-q_{4}\right)}{\partial \ell_{4}}}
$$


By Lemma $10.2(\mathrm{c})$, we know that the angle formed by $q_{3}-q_{4}$ and $p_{3}-p_{4}$ is $O\left(\mu^{1-\kappa}\right)$. Thus, in (10.13), we can replace $q_{3}-q_{4}$ by $p_{3}-p_{4}$ making an error of $O\left(\mu^{1-\kappa}\right)$. Hence,

$$
\nabla \mathcal{V} \ell_{4}=\frac{\left(p_{3}-p_{4}\right) \cdot \nabla \mathcal{V}\left(q_{3}-q_{4}\right)}{\left(p_{3}-p_{4}\right) \cdot \partial q_{4} / \partial \ell_{4}}+O\left(\mu^{1-\kappa}\right)
$$

Note that $\partial q_{4} / \partial \ell_{4}$ is parallel to $p_{4}$. Using the information about $v_{3}$ and $v_{4}$ from Appendix B.1, we see that $\left\langle v_{3}, v_{4}\right\rangle \neq\left\langle v_{4}, v_{4}\right\rangle$. Therefore, the denominator in (10.13) is bounded away from zero, and so

$$
\nabla \mathcal{V} \ell_{4}=O\left(1,1,1,1 ; \mu^{1-\kappa}, \mu^{1-\kappa}, \mu^{1-\kappa}, \mu^{1-\kappa} ; 1,1\right)
$$

We also need to make sure the second component $\nabla_{\ell_{3}} \ell_{4}$ is not close to -1 , so that

$$
\mathrm{Id}-\mathcal{F} \otimes \nabla \mathcal{V} \ell_{4}
$$

is invertible on the section $\left|q_{3}-q_{4}\right|=\mu^{\kappa}$. In fact, we have

$$
\nabla_{\ell_{3}} \ell_{4} \rightarrow-\frac{\left(p_{3}-p_{4}\right) \cdot p_{3}}{\left(p_{3}-p_{4}\right) \cdot p_{4}}
$$

as $\mu \rightarrow 0$. The fact that $\nabla_{\ell_{3}} \ell_{4}$ is not close to -1 is then verified using the information in Appendix B.1.

For the derivative $d \widetilde{\mathbb{G}}$, the fundamental solution is estimated as $\operatorname{Id}+c e_{2,1}+o(1)$, and the two boundary terms are both given by the estimates (8.3) and Corollary 6.10 on the section $\left\{x_{4, \|}^{R}=-2\right\}$. Now, the statement of the lemma can be checked with this explicit information.

This lemma shows that we can replace $\mathbb{G}$ by $\mathbb{L}^{-} \circ \mathbb{G}_{\circ} \mathbb{L}^{+}$, and the bound of Lemma 3.2 still holds. So, in the following it is enough to study $\mathbb{L}^{0}$ instead of $\mathbb{L}$ to prove Lemma 3.1.

Proof of Lemma 3.1. As before, we use the formula (7.1). We need to consider the integration of the variational equations and also the boundary contribution.

Recall that the subscripts - and + mean relative motion and center of mass motion, respectively, and the superscripts - and + mean incoming and outgoing, respectively. In the following, we are most interested in the relative motion, so we drop the subscript of $q_{-}, p_{-}, \mathcal{L}_{-}, G_{-}$and $g_{-}$for simplicity without leading to confusion. Note that, in the following discussion, the symbol $q_{1}$ has two different meanings, but it is always clear from context. If $q_{1}$ and $q_{2}$ both appear in an equation, then they mean the horizontal and vertical components respectively of $q_{-}$. If $q_{1}$ appears in $\mathbf{q}$ or with $p_{1}$ without $q_{2}$, then it means $Q_{1}-Q_{2}$, the position of body 1 . 
Step 1. (The Hamiltonian equations, the variational equations and the boundary contributions.)

It is convenient to use the variable $\mathcal{L}=L / \mu$. Lemma 10.2 says that $1 / c<\mathcal{L}<c$ and $\mu / c \leqslant G \leqslant c \mu$ for some $c>1$, if the rotation angle $\alpha$ is bounded away from zero and $\pi$. We also have $g, \mathbf{q}, \mathbf{p}, p=O(1)$ and $q=O\left(\mu^{\kappa}\right)$. From the Hamiltonian (4.10), we have

$$
\dot{\ell}=-\frac{1}{2 \mu \mathcal{L}^{3}}+O\left(\mu^{2 \kappa-1}\right)
$$

(see (10.8)). Using $\ell$ as the time variable, we get from (4.10) that the equations of motion take the following form (recall that $\ell=O\left(\mu^{\kappa-1}\right)$ due to $(10.7)$ ):

$$
\left\{\begin{array} { l } 
{ \frac { d \mathcal { L } } { d \ell } = - \mu ^ { - 1 } \frac { d t } { d \ell } \frac { \partial H } { \partial \ell } \sim \mu ^ { 1 + \kappa } , } \\
{ \frac { d G } { d \ell } = - \frac { d t } { d \ell } \frac { \partial H } { \partial g } \sim \mu ^ { 1 + 2 \kappa } } \\
{ \frac { d g } { d \ell } = \frac { d t } { d \ell } \frac { \partial H } { \partial G } \sim \mu ^ { 2 \kappa } }
\end{array} \quad \left\{\begin{array}{l}
\frac{d q_{+}}{d \ell}=\frac{d t}{d \ell}\left(\frac{1+2 \mu}{2} p_{+}+\mu p_{1}\right) \sim \mu \\
\frac{d p_{+}}{d \ell}=\frac{d t}{d \ell}\left(\frac{2 q_{+}}{\left|q_{+}\right|^{3}}+O\left(\mu^{2 \kappa}+\frac{1}{\chi^{2}}\right)\right) \sim \mu \\
\frac{d q_{1}}{d \ell}=\frac{d t}{d \ell}\left(\frac{1}{2} \mu p_{1}+\mu p_{+}\right) \sim \mu^{2} \\
\frac{d p_{1}}{d \ell}=\frac{d t}{d \ell}\left(\frac{1+2 \mu q_{1}}{\mu\left|q_{1}\right|^{3}}+O\left(\frac{1}{\chi^{3}}\right)\right) \sim \frac{1}{\chi^{2}}
\end{array}\right.\right.
$$

and we have

$$
\frac{d}{d \ell}(q, p)=O(\mu)
$$

In the first three equations, the main contribution to $H$ comes from $|q|^{2}$ and $\left|q_{+} \cdot q\right|^{2}$, both of which are $O\left(\mu^{2 \kappa}\right)$. We have the estimate

$$
\left|\left(\frac{\partial}{\partial \mathcal{L}}, \frac{\partial}{\partial \ell}, \frac{\partial}{\partial G}, \frac{\partial}{\partial g}\right) q\right|=O\left(\mu^{\kappa}, \mu, \mu^{\kappa-1}, \mu^{\kappa}\right),
$$

using (10.6) for $q=\left(q_{1}, q_{2}\right)$, up to a rotation by $g$. In fact, the $\partial / \partial \ell$ amounts to dividing by the scale of $\ell$, i.e. $\mu^{-1+\kappa}$. The derivatives $\partial / \partial \mathcal{L}$ and $\partial / \partial g$ do not change the order of magnitude. Finally, since $G=O(\mu)$, the $\partial / \partial G$ amounts to dividing by $\mu$.

Next, we analyze the variational equations. The same rules as those used to obtain (10.14) apply here. We have

$$
\frac{d}{d \ell}\left[\begin{array}{c}
\delta \mathcal{L} \\
\delta G \\
\delta g \\
\delta \mathbf{q} \\
\delta \mathbf{p}
\end{array}\right]=O\left(\begin{array}{ccccc}
\mu^{1+\kappa} & \mu^{\kappa} & \mu^{1+\kappa} & \mu^{1+\kappa} & 0 \\
\mu^{1+2 \kappa} & \mu^{2 \kappa} & \mu^{1+2 \kappa} & \mu^{1+2 \kappa} & 0 \\
\mu^{2 \kappa} & \mu^{2 \kappa-1} & \mu^{2 \kappa} & \mu^{2 \kappa} & 0 \\
\mu & \mu^{2 \kappa} & \mu^{2 \kappa+1} & \mu^{2 \kappa+1} & \mu \\
\mu & \mu^{2 \kappa} & \mu^{2 \kappa+1} & \mu & 0
\end{array}\right)\left[\begin{array}{c}
\delta \mathcal{L} \\
\delta G \\
\delta g \\
\delta \mathbf{q} \\
\delta \mathbf{p}
\end{array}\right] .
$$


We need to integrate this equation over time $\mu^{\kappa-1}$. Thus, we compare the solution to the variational equation with a constant linear ODE of the form $X^{\prime}=A X$. Its solution has the form

$$
X\left(\mu^{\kappa-1}\right)=\sum_{n=0}^{\infty} \frac{\left(A \mu^{\kappa-1}\right)^{n}}{n !} .
$$

We will show that $\left(A \mu^{\kappa-1}\right)^{3} \leqslant C_{3}\left(\left(A \mu^{\kappa-1}\right)+\left(A \mu^{\kappa-1}\right)^{2}\right)$. Then, we have

$$
\left(A \mu^{\kappa-1}\right)^{n} \leqslant C_{n}\left(\left(A \mu^{\kappa-1}\right)+\left(A \mu^{\kappa-1}\right)^{2}\right) \quad \text { and } \quad C_{n}=C_{3}\left(1+C_{3}\right)^{n} .
$$

Hence, $X\left(\mu^{\kappa-1}\right) \leqslant \operatorname{Id}+C\left(\left(A \mu^{\kappa-1}\right)+\left(A \mu^{\kappa-1}\right)^{2}\right)$. We next integrate the variational equations over time $O\left(\mu^{\kappa-1}\right)$ to get the estimate of its fundamental solution

$$
\operatorname{Id}_{11}+O\left(\begin{array}{ccc|cc}
\mu^{2 \kappa} & \mu^{2 \kappa-1} & \mu^{2 \kappa} & \mu^{2 \kappa} & \mu^{3 \kappa} \\
\mu^{3 \kappa} & \mu^{3 \kappa-1} & \mu^{3 \kappa} & \mu^{3 \kappa} & \mu^{4 \kappa} \\
\mu^{3 \kappa-1} & \mu^{3 \kappa-2} & \mu^{3 \kappa-1} & \mu^{3 \kappa-1} & \mu^{4 \kappa-1} \\
\hline \mu^{\kappa} & \mu^{3 \kappa-1} & \mu^{3 \kappa} & \mu^{2 \kappa} & \mu^{\kappa} \\
\mu^{\kappa} & \mu^{3 \kappa-1} & \mu^{3 \kappa} & \mu^{\kappa} & \mu^{2 \kappa}
\end{array}\right) .
$$

Next, we compute the boundary contribution using the formula (7.1). In terms of the Delaunay variables inside the circle $|q|=\frac{1}{2} \mu^{\kappa}$, we have

$$
\frac{\partial \ell}{\partial(\mathcal{L}, G, g, \mathbf{q}, \mathbf{p})}=-\left(\frac{\partial|q|}{\partial \ell}\right)^{-1} \frac{\partial|q|}{\partial(\mathcal{L}, G, g, \mathbf{q}, \mathbf{p})}=\left(O\left(\mu^{\kappa-1}\right), O\left(\mu^{\kappa-2}\right), 0,0,0\right)
$$

Indeed, due to (10.6), we have

$$
\frac{\partial|q|}{\partial g}=0, \quad \frac{\partial|q|}{\partial \ell}=O(\mu), \quad \frac{\partial|q|}{\partial \mathcal{L}}=O\left(\mu^{\kappa}\right) \quad \text { and } \quad \frac{\partial|q|}{\partial G}=O\left(\mu^{\kappa-1}\right) .
$$

Combining this with (10.14), we get

$$
\left(\frac{\partial}{\partial \ell}(\mathcal{L}, G, g, \mathbf{q}, \mathbf{p})\right) \otimes \frac{\partial \ell}{\partial(\mathcal{L}, G, g, \mathbf{q}, \mathbf{p})}=O\left(\mu^{1+\kappa}, \mu^{1+2 \kappa}, \mu^{2 \kappa}, \mu, \mu\right) \otimes O\left(\mu^{\kappa-1}, \mu^{\kappa-2}, 0,0,0\right)
$$

Step 2. (The analysis of the relative motion part.)

The structure of $d \mathbb{L}_{0}$ comes mainly from the relative motion part, on which we now focus. We neglect the $\mathbf{q}$ and $\mathbf{p}$ part, and will study it in the last step. 
Substep 2.1. (The strategy.)

Using (7.1), we obtain the derivative matrix

$$
\begin{aligned}
\frac{\partial(\mathcal{L}, G, g)^{+}}{\partial(\mathcal{L}, G, g)^{-}}= & \left.\operatorname{Id}_{3}+O\left(\begin{array}{ccc}
\mu^{2 \kappa} & \mu^{2 \kappa-1} & 0 \\
\mu^{3 \kappa} & \mu^{3 \kappa-1} & 0 \\
\mu^{3 \kappa-1} & \mu^{3 \kappa-2} & 0
\end{array}\right)\right)^{-1} \\
& \times\left(\operatorname{Id}_{3}+O\left(\begin{array}{ccc}
\mu^{2 \kappa} & \mu^{2 \kappa-1} & \mu^{2 \kappa} \\
\mu^{3 \kappa} & \mu^{3 \kappa-1} & \mu^{3 \kappa} \\
\mu^{3 \kappa-1} & \mu^{3 \kappa-2} & \mu^{3 \kappa-1}
\end{array}\right)\right) \\
& \times\left(\operatorname{Id}_{3}-O\left(\begin{array}{ccc}
\mu^{2 \kappa} & \mu^{2 \kappa-1} & 0 \\
\mu^{3 \kappa} & \mu^{3 \kappa-1} & 0 \\
\mu^{3 \kappa-1} & \mu^{3 \kappa-2} & 0
\end{array}\right)\right) \\
= & \operatorname{Id}_{3}+O\left(\begin{array}{ccc}
\mu^{2 \kappa} & \mu^{2 \kappa-1} & \mu^{2 \kappa} \\
\mu^{3 \kappa} & \mu^{3 \kappa-1} & \mu^{3 \kappa} \\
\mu^{3 \kappa-1} & \mu^{3 \kappa-2} & \mu^{3 \kappa-1}
\end{array}\right) \\
= & \operatorname{Id}_{3}+P .
\end{aligned}
$$

For the position variables $q$, we are only interested in the angle $\Theta:=\arctan \left(q_{2} / q_{1}\right)$, since the length $\left|\left(q_{1}, q_{2}\right)\right|=\frac{1}{2} \mu^{\kappa}$ is fixed when restricted to the circle. We split the derivative matrix as follows:

$$
\begin{aligned}
\frac{\partial(\Theta, p)^{+}}{\partial(\Theta, p)^{-}} & =\frac{\partial(\Theta, p)^{+}}{\partial(\mathcal{L}, G, g)^{+}} \frac{\partial(\mathcal{L}, G, g)^{+}}{\partial(\mathcal{L}, G, g)^{-}} \frac{\partial(\mathcal{L}, G, g)^{-}}{\partial(\Theta, p)^{-}} \\
& =\frac{\partial(\Theta, p)^{+}}{\partial(\mathcal{L}, G, g)^{+}} \frac{\partial(\mathcal{L}, G, g)^{-}}{\partial(\Theta, p)^{-}}+\frac{\partial(\Theta, p)^{+}}{\partial(\mathcal{L}, G, g)^{+}} P \frac{\partial(\mathcal{L}, G, g)^{-}}{\partial(\Theta, p)^{-}} \\
& =: \mathrm{I}+\mathrm{II} .
\end{aligned}
$$

Now, we prove the following claim.

Claim.

$$
\begin{aligned}
\mathrm{I} & =\frac{1}{\mu} O(1)_{1 \times 3} \otimes \frac{\partial G^{-}}{\partial(\Theta, p)^{-}}+O(1), \\
\mathrm{II} & =\frac{1}{\mu} O\left(\mu^{3 \kappa-1}\right)_{1 \times 3} \otimes \frac{\partial G^{-}}{\partial(\Theta, p)^{-}}+O\left(\mu^{3 \kappa-1}\right) .
\end{aligned}
$$

We will give the expressions of $O(1)$ terms explicitly. 
Substep 2.2. (The estimate of I in the splitting (10.20).)

Using equations (10.6) and (10.10), we obtain

$$
\frac{\partial(\Theta, p)^{+}}{\partial(\mathcal{L}, G, g)^{+}}=O\left(\begin{array}{ccc}
1 & \mu^{-1} & 1 \\
1 & \mu^{-1} & 1 \\
1 & \mu^{-1} & 1
\end{array}\right)
$$

Next, we consider the first term in (10.20),

$$
\mathrm{I}=\frac{\partial(\Theta, p)^{+}}{\partial \mathcal{L}^{+}} \otimes \frac{\partial \mathcal{L}^{-}}{\partial(\Theta, p)^{-}}+\frac{\partial(\Theta, p)^{+}}{\partial G^{+}} \otimes \frac{\partial G^{-}}{\partial(\Theta, p)^{-}}+\frac{\partial(\Theta, p)^{+}}{\partial g^{+}} \otimes \frac{\partial g^{-}}{\partial(\Theta, p)^{-}} .
$$

Using the expressions

$$
\frac{1}{4 \mathcal{L}^{2}}=\frac{p^{2}}{4}-\frac{\mu}{2|q|} \quad \text { and } \quad G=p \times q=|p| \cdot|q| \sin \measuredangle(p, q)
$$

we see that

$$
\frac{\partial \mathcal{L}^{-}}{\partial(\Theta, p)^{-}}=O(1) \quad \text { and } \quad \frac{\partial G^{-}}{\partial(\Theta, p)^{-}}=\left(O\left(\mu^{\kappa}\right), O\left(\mu^{\kappa}\right)\right)
$$

It only remains to get the estimate of $\partial g^{-} / \partial(\Theta, p)^{-}$. We claim that

$$
\frac{\partial g^{-}}{\partial(\Theta, p)^{-}}=\left[\frac{\partial}{\partial G^{-}} \arctan \left(\frac{G^{-}}{\mu \mathcal{L}}\right)\right] \frac{\partial G^{-}}{\partial(\Theta, p)^{-}}+O(1)=O\left(\frac{1}{\mu}\right) \frac{\partial G^{-}}{\partial(\Theta, p)^{-}}+O(1) .
$$

We use equation (10.11) to get

$$
g=\arctan \left(\frac{p_{2}}{p_{1}}-e^{-2|u|} E\left(\frac{G}{\mu \mathcal{L}}, g, u\right)\right)-\operatorname{sign}(u) \arctan \left(\frac{G}{\mu \mathcal{L}}\right) \quad \text { as }|u| \rightarrow \infty .
$$

We have

$$
e^{-2|u|} \sim \frac{1}{\ell^{2}}\left(1+\left(\frac{G}{\mu \mathcal{L}}\right)^{2}\right) \sim\left(1+\left(\frac{G}{\mu \mathcal{L}}\right)^{2}\right) \mu^{2(1-\kappa)} \mathcal{L}^{4},
$$

using (10.6) and (A.4), and $E(\cdot, \cdot, \cdot)$ has $O(1)$ derivatives as $|u| \rightarrow \infty$. Thus, we get

$$
\begin{aligned}
\frac{\partial g}{\partial(\Theta, p)}\left(1+O\left(e^{-2|u|}\right)\right)= & \frac{\partial \arctan \left(p_{2} / p_{1}\right)}{\partial(\Theta, p)}+\left(\frac{\partial \arctan (G / \mu \mathcal{L})}{\partial \mathcal{L}}+O\left(e^{-2|u|}\right)\right) \frac{\partial \mathcal{L}}{\partial(\Theta, p)} \\
& +\left(\frac{\partial \arctan (G / \mu \mathcal{L}}{\partial G}+O\left(\frac{e^{-2|u|}}{\mu}\right)\right) \frac{\partial G}{\partial(\Theta, p)}+O\left(e^{-2|u|}\right),
\end{aligned}
$$

proving (10.25).

Plugging (10.22), (10.24) and (10.25) back into (10.23), we get the estimate of $I$ in (10.21). More explicitly,

$$
I=\frac{1}{\mu} \mathbf{U} \otimes \frac{\partial G^{-}}{\partial(\Theta, p)^{-}}+\mathbf{B},
$$


where

$$
\begin{aligned}
& \mathbf{U}=\mu \frac{\partial(\Theta, p)^{+}}{\partial G^{+}}+\mu \frac{\partial \arctan \left(G^{-} / \mu \mathcal{L}^{-}\right)}{\partial G^{-}} \frac{\partial(\Theta, p)^{+}}{\partial g^{+}}+O\left(\mu^{1-\kappa}\right) \\
& \mathbf{B}=\frac{\partial(\Theta, p)^{+}}{\partial \mathcal{L}^{+}} \otimes \frac{\partial \mathcal{L}^{-}}{\partial(\Theta, p)^{-}} \\
& \quad+\frac{\partial(\Theta, p)^{+}}{\partial g^{+}} \otimes\left(\frac{\partial \arctan \left(p_{2}^{-} / p_{1}^{-}\right)}{\partial(\Theta, p)^{-}}+\frac{\partial \arctan \left(G^{-} / \mu \mathcal{L}^{-}\right)}{\partial \mathcal{L}^{-}} \frac{\partial \mathcal{L}^{-}}{\partial(\Theta, p)^{-}}\right) \\
& \quad+O\left(\mu^{1-\kappa}\right) .
\end{aligned}
$$

Substep 2.3. (The estimate of II in the splitting (10.20).)

Now we study the second term in (10.20):

$$
\begin{aligned}
\mathrm{II} & =O\left(\begin{array}{lll}
1 & \mu^{-1} & 1 \\
1 & \mu^{-1} & 1 \\
1 & \mu^{-1} & 1
\end{array}\right) \cdot O\left(\begin{array}{ccc}
\mu^{2 \kappa} & \mu^{2 \kappa-1} & \mu^{2 \kappa} \\
\mu^{3 \kappa} & \mu^{3 \kappa-1} & \mu^{3 \kappa} \\
\mu^{3 \kappa-1} & \mu^{3 \kappa-2} & \mu^{3 \kappa-1}
\end{array}\right) \frac{\partial(\mathcal{L}, G, g)^{-}}{\partial(\Theta, p)^{-}} \\
& =O\left(\begin{array}{lll}
\mu^{3 \kappa-1} & \mu^{3 \kappa-2} & \mu^{3 \kappa-1} \\
\mu^{3 \kappa-1} & \mu^{3 \kappa-2} & \mu^{3 \kappa-1} \\
\mu^{3 \kappa-1} & \mu^{3 \kappa-2} & \mu^{3 \kappa-1}
\end{array}\right) \frac{\partial(\mathcal{L}, G, g)^{-}}{\partial(\Theta, p)^{-}} \\
& =\mu^{3 \kappa-1}\left[O(1)_{1 \times 3} \otimes \frac{\partial \mathcal{L}^{-}}{\partial(\Theta, p)^{-}}+O\left(\mu^{-1}\right)_{1 \times 3} \otimes \frac{\partial G^{-}}{\partial(\Theta, p)^{-}}+O(1)_{1 \times 3} \otimes \frac{\partial g^{-}}{\partial(\Theta, p)^{-}}\right]
\end{aligned}
$$

where we use that $\mu^{2 \kappa}<\mu^{3 \kappa-1}$ and $\mu^{2 \kappa-1}<\mu^{3 \kappa-2}$, since $\kappa<\frac{1}{2}$. The first summand in $(10.27)$ is $O\left(\mu^{3 \kappa-1}\right)$. Applying (10.25), we get the estimate of II in (10.21). We then obtain

$$
\mathrm{I}+\mathrm{II}=\frac{1}{\mu}\left(\mathbf{U}+O\left(\mu^{3 \kappa-1}\right)\right) \otimes \frac{\partial G^{-}}{\partial(\Theta, p)^{-}}+\mathbf{B}+O\left(\mu^{3 \kappa-1}\right) .
$$

Substep 2.4. (Going from $\Theta$ to $q$.)

We use the variable $\Theta$ for the relative position $q$ and we have

$$
\begin{gathered}
\frac{\partial G^{-}}{\partial(\Theta, p)^{-}}=O\left(\mu^{\kappa}\right), \quad q=\frac{1}{2} \mu^{\kappa}(\cos \Theta, \sin \Theta)=(x, y) \\
\Theta=\arctan \left(\frac{y}{x}\right), \quad|q| d \Theta=-(\sin \Theta) d x+(\cos \Theta) d y
\end{gathered}
$$

So, we have the estimate

$$
\frac{\partial q^{+}}{\partial(\mathcal{L}, G, g)^{+}}=O\left(\mu^{\kappa}\right) \frac{\partial \Theta^{+}}{\partial(\mathcal{L}, G, g)^{+}}=O\left(\mu^{\kappa-1}\right) .
$$


To get $\partial \cdot / \partial q^{-}$, we transform polar coordinates to Cartesian,

$$
\frac{\partial \cdot}{\partial q^{-}}=\frac{\partial \cdot}{\partial(r, \Theta)^{-}} \frac{\partial(r, \Theta)^{-}}{\partial q^{-}}
$$

where $r=\left|q^{-}\right|=\frac{1}{2} \mu^{\kappa}$. Therefore, we have

$$
\frac{\partial r^{-}}{\partial q^{-}}=0 \quad \text { and } \quad \frac{\partial \cdot}{\partial q^{-}}=\frac{2}{\mu^{\kappa}} \frac{\partial \cdot}{\partial \Theta^{-}}\left(-\sin \Theta^{-}, \cos \Theta^{-}\right) .
$$

So, we have the estimate $\partial G^{-} / \partial q^{-}=O(1)$, and

$$
\frac{\partial \mathcal{L}^{-}}{\partial q^{-}}=\frac{\partial \mathcal{L}^{-}}{\partial \Theta^{-}}=0
$$

since in the expression

$$
\frac{1}{4 \mathcal{L}^{2}}=\frac{p^{2}}{4}-\frac{\mu}{2|q|}
$$

the angle $\Theta$ plays no role. Finally, we have

$$
\frac{\partial}{\partial q^{-}} \arctan \left(\frac{p_{2}^{-}}{p_{1}^{-}}\right)=0
$$

Applying these estimates to (10.26) and (10.27), we get

$$
\frac{\partial(q, p)^{+}}{\partial(q, p)^{-}}=\frac{1}{\mu}\left(O\left(\mu^{\kappa}\right)_{1 \times 2}, O(1)_{1 \times 2}\right) \otimes\left(O(1)_{1 \times 2}, O\left(\mu^{\kappa}\right)_{1 \times 2}\right)+O(1)_{4 \times 4} .
$$

Step 3. (The contribution from the motion of the center of mass.)

Substep 3.1. (The decomposition.)

Consider the following decomposition:

$$
\begin{aligned}
\mathcal{D}:= & \frac{\partial(\Theta, p, \mathbf{q}, \mathbf{p})^{+}}{\partial(\Theta, p, \mathbf{q}, \mathbf{p})^{-}}=\frac{\partial(\Theta, p ; \mathbf{q}, \mathbf{p})^{+}}{\partial(\mathcal{L}, G, g ; \mathbf{q}, \mathbf{p})^{+}} \frac{\partial(\mathcal{L}, G, g ; \mathbf{q}, \mathbf{p})^{+}}{\partial(\mathcal{L}, G, g ; \mathbf{q}, \mathbf{p})\left(\ell^{\mathrm{f}}\right)} \\
& \times \frac{\partial(\mathcal{L}, G, g ; \mathbf{q}, \mathbf{p})\left(\ell^{\mathrm{f}}\right)}{\partial(\mathcal{L}, G, g ; \mathbf{q}, \mathbf{p})\left(\ell^{\mathrm{i}}\right)} \frac{\partial(\mathcal{L}, G, g ; \mathbf{q}, \mathbf{p})\left(\ell^{\mathrm{i}}\right)}{\partial(\mathcal{L}, G, g ; \mathbf{q}, \mathbf{p})^{-}} \frac{\partial(\mathcal{L}, G, g ; \mathbf{q}, \mathbf{p})^{-}}{\partial(\Theta, p ; \mathbf{q}, \mathbf{p})^{-}} \\
= & :\left[\begin{array}{cc}
M & 0 \\
0 & \mathrm{Id}_{8}
\end{array}\right]\left[\begin{array}{cc}
A & 0 \\
B & \mathrm{Id}_{8}
\end{array}\right]\left[\begin{array}{cc}
C & D \\
E & F
\end{array}\right]\left[\begin{array}{cc}
A^{\prime} & 0 \\
B^{\prime} & \mathrm{Id}_{8}
\end{array}\right]\left[\begin{array}{cc}
N & 0 \\
0 & \mathrm{Id}_{8}
\end{array}\right] \\
= & {\left[\begin{array}{cc}
M A C A^{\prime} N+M A D B^{\prime} N & M A D \\
(B C+E) A^{\prime} N+(B D+F) B^{\prime} N & B D+F
\end{array}\right] . }
\end{aligned}
$$

Each of the above matrices is $11 \times 11$. 
Substep 3.2. (The estimate of each block.)

The matrix

$$
M=\frac{\partial(\Theta, p)^{+}}{\partial(\mathcal{L}, G, g)^{+}}
$$

is given by (10.22) and

$$
N=\frac{\partial(\mathcal{L}, G, g)^{-}}{\partial(\Theta, p)^{-}}
$$

by (10.24) and (10.25):

$$
M=O\left(\begin{array}{lll}
1 & \mu^{-1} & 1 \\
1 & \mu^{-1} & 1 \\
1 & \mu^{-1} & 1
\end{array}\right) \quad \text { and } \quad N=\left(\begin{array}{c}
O(1)_{1 \times 3} \\
\frac{\partial G^{-}}{\partial(\Theta, p)^{-}} \\
O\left(\frac{1}{\mu}\right) \frac{\partial G^{-}}{\partial(\Theta, p)^{-}}+O(1)
\end{array}\right) .
$$

The matrices $C, D, E$ and $F$ form the matrix (10.16), the fundamental solution of the variational equation,

$$
\left(\begin{array}{c|c}
C & D \\
\hline E & F
\end{array}\right)=\operatorname{Id}_{11}+O\left(\begin{array}{ccc|cc}
\mu^{2 \kappa} & \mu^{2 \kappa-1} & \mu^{2 \kappa} & \left(\mu^{2 \kappa}\right)_{1 \times 4} & \left(\mu^{3 \kappa}\right)_{1 \times 4} \\
\mu^{3 \kappa} & \mu^{3 \kappa-1} & \mu^{3 \kappa} & \left(\mu^{3 \kappa}\right)_{1 \times 4} & \left(\mu^{4 \kappa}\right)_{1 \times 4} \\
\mu^{3 \kappa-1} & \mu^{3 \kappa-2} & \mu^{3 \kappa-1} & \left(\mu^{3 \kappa-1}\right)_{1 \times 4} & \left(\mu^{4 \kappa-1}\right)_{1 \times 4} \\
\hline\left(\mu^{\kappa}\right)_{4 \times 1} & \left(\mu^{3 \kappa-1}\right)_{4 \times 1} & \left(\mu^{3 \kappa}\right)_{4 \times 1} & \left(\mu^{2 \kappa}\right)_{4 \times 4} & \left(\mu^{\kappa}\right)_{4 \times 4} \\
\left(\mu^{\kappa}\right)_{4 \times 1} & \left(\mu^{3 \kappa-1}\right)_{4 \times 1} & \left(\mu^{3 \kappa}\right)_{4 \times 1} & \left(\mu^{\kappa}\right)_{4 \times 4} & \left(\mu^{2 \kappa}\right)_{4 \times 4}
\end{array}\right) .
$$

The matrices $A, B, A^{\prime}$ and $B^{\prime}$ are given by (10.18), boundary contributions,

$$
\left[\begin{array}{c|c}
A & 0 \\
\hline B & \mathrm{Id}_{8}
\end{array}\right],\left[\begin{array}{c|c}
A^{\prime} & 0 \\
\hline B^{\prime} & \mathrm{Id}_{8}
\end{array}\right]=\operatorname{Id}_{11}+O\left(\mu^{1+\kappa}, \mu^{1+2 \kappa}, \mu^{2 \kappa} ; \mu_{1 \times 8}\right) \otimes O\left(\mu^{\kappa-1}, \mu^{\kappa-2}, 0 ; 0_{1 \times 8}\right) .
$$

Substep 3.3. (The estimate of the first block $M A C A^{\prime} N+M A D B^{\prime} N$ in $\mathcal{D}$.)

By (10.19),

$$
A C A^{\prime}=\operatorname{Id}_{3}+P=\operatorname{Id}_{3}+O\left(\begin{array}{ccc}
\mu^{2 \kappa} & \mu^{2 \kappa-1} & \mu^{2 \kappa} \\
\mu^{3 \kappa} & \mu^{3 \kappa-1} & \mu^{3 \kappa} \\
\mu^{3 \kappa-1} & \mu^{3 \kappa-2} & \mu^{3 \kappa-1}
\end{array}\right)
$$

(recall that $(10.19)$ is the part of $\partial(\mathcal{L}, G, g)^{+} / \partial(\mathcal{L}, G, g)^{-}$without considering the motion of the center of mass), and by (10.21) and (10.26),

$$
M A C A^{\prime} N=M\left(\operatorname{Id}_{3}+P\right) N=\frac{1}{\mu}\left(\mathbf{U}+O\left(\mu^{3 \kappa-1}\right)\right) \otimes \frac{\partial G^{-}}{\partial(\Theta, p)^{-}}+\mathbf{B}+O\left(\mu^{3 \kappa-1}\right) .
$$


Indeed, using the notation of (10.20), we have $\mathrm{I}=M N$ and $\mathrm{II}=M P N$. The estimates of $\mathrm{I}$ and II are given in (10.21).

Next, we claim that

$$
M A D B^{\prime} N=O\left(\mu^{3 \kappa-2}\right) \frac{\partial G^{-}}{\partial(\Theta, p)^{-}}+O\left(\mu^{3 \kappa-1}\right)
$$

so it can be absorbed into the error terms of (10.30). To this end, we split $N=N_{1}+N_{2}$ and $A=\mathrm{Id}+A_{2}$, where $A_{2}=O\left(\mu^{1+\kappa}, \mu^{1+2 \kappa}, \mu^{2 \kappa}\right) \otimes O\left(\mu^{\kappa-1}, \mu^{\kappa-2}, 0\right)$ and

$$
N_{1}=\left(\begin{array}{c}
0_{1 \times 3} \\
\frac{\partial G^{-}}{\partial(\Theta, p)^{-}} \\
O\left(\frac{1}{\mu}\right) \frac{\partial G^{-}}{\partial(\Theta, p)^{-}}
\end{array}\right) \quad \text { and } \quad N_{2}=\left(\begin{array}{c}
O(1)_{1 \times 3} \\
0_{1 \times 3} \\
O(1)_{1 \times 3}
\end{array}\right)
$$

Thus, $M A D B^{\prime} N=M D B^{\prime} N+M A_{2} D B^{\prime} N$. Let us work on the first term. A direct computation shows that

$$
D B^{\prime}=O\left(\begin{array}{ccc}
\mu^{3 \kappa} & \mu^{3 \kappa-1} & 0 \\
\mu^{4 \kappa} & \mu^{4 \kappa-1} & 0 \\
\mu^{4 \kappa-1} & \mu^{4 \kappa-2} & 0
\end{array}\right) \quad \text { and } \quad M D B^{\prime}=O\left(\mu_{3 \times 1}^{4 \kappa-1}, \mu_{3 \times 1}^{4 \kappa-2}, 0_{3 \times 1}\right)
$$

Now, it is easy to see that $M D B^{\prime} N_{1}$ can be absorbed into the first term in (10.31) and $M D B^{\prime} N_{2}$ can be absorbed into the second term. The key is that $N_{1}$ has rank 1 and the second row of $N_{2}$ is zero. The analysis of $M A_{2} D B^{\prime} N$ is even easier, since a direct computation shows that $D B^{\prime}$ dominates $A_{2} D B^{\prime}$ componentwise. This proves (10.31) and shows that $M A C A^{\prime} N+M A D B^{\prime} N$ has the same asymptotics as (10.30).

Substep 3.4. (Estimate of the remaining blocks in $\mathcal{D}$.)

The following estimates are obtained by a direct computation:

$$
\begin{aligned}
B D+F= & \left(\mu_{1 \times 8}\right) \otimes O\left(\mu^{\kappa-1}, \mu^{\kappa-2}, 0\right) O\left(\begin{array}{cc}
\left(\mu^{2 \kappa}\right)_{1 \times 4} & \left(\mu^{3 \kappa}\right)_{1 \times 4} \\
\left(\mu^{3 \kappa}\right)_{1 \times 4} & \left(\mu^{4 \kappa}\right)_{1 \times 4} \\
\left(\mu^{3 \kappa-1}\right)_{1 \times 4} & \left(\mu^{4 \kappa-1}\right)_{1 \times 4}
\end{array}\right) \\
& +\operatorname{Id}_{8}+O\left(\begin{array}{cc}
\left(\mu^{2 \kappa}\right)_{4 \times 4} & \left(\mu^{\kappa}\right)_{4 \times 4} \\
\left(\mu^{\kappa}\right)_{4 \times 4} & \left(\mu^{2 \kappa}\right)_{4 \times 4}
\end{array}\right) \\
= & \operatorname{Id}_{8}+O\left(\mu^{\kappa}\right)_{8 \times 8} .
\end{aligned}
$$


and

$$
\begin{aligned}
B C+E= & O\left(\mu_{1 \times 8}\right) \otimes O\left(\mu^{\kappa-1}, \mu^{\kappa-2}, 0\right) O\left(\begin{array}{ccc}
\mu^{2 \kappa} & \mu^{2 \kappa-1} & \mu^{2 \kappa} \\
\mu^{3 \kappa} & \mu^{3 \kappa-1} & \mu^{3 \kappa} \\
\mu^{3 \kappa-1} & \mu^{3 \kappa-2} & \mu^{3 \kappa-1}
\end{array}\right) \\
& \quad+\left(\left(\mu^{\kappa}\right)_{8 \times 1},\left(\mu^{3 \kappa-1}\right)_{8 \times 1},\left(\mu^{3 \kappa}\right)_{8 \times 1}\right) \\
= & O\left(\left(\mu^{\kappa}\right)_{8 \times 1},\left(\mu^{4 \kappa-2}\right)_{8 \times 1},\left(\mu^{4 \kappa-1}\right)_{8 \times 1}\right) .
\end{aligned}
$$

Accordingly, using (10.24) and (10.25) for $N$, and arguing the same way as in Substep 3.3, we get

$$
\begin{aligned}
(B C+E) A^{\prime} N+(B D+F) B^{\prime} N & =\frac{1}{\mu}\left[O\left(\mu^{\kappa}\right)\right]_{1 \times 8} \otimes \frac{\partial G^{-}}{\partial(\Theta, p)_{-}^{-}}+O\left(\mu^{\kappa}\right), \\
M A D & =\left[O\left(\mu^{3 \kappa-1}\right)\right]_{3 \times 8} .
\end{aligned}
$$

Substep 3.5. (Completing the asymptotics of $\mathcal{D}$.)

Substeps 3.1-3.4 above can be summarized as follows:

$$
\mathcal{D}=\frac{1}{\mu}\left(\mathbf{U}+O\left(\mu^{3 \kappa-1}\right) ; O\left(\mu^{\kappa}\right)_{1 \times 8}\right) \otimes\left(\frac{\partial G^{-}}{\partial(\Theta, p)_{-}^{-}} ; 0_{1 \times 8}\right)+\left(\begin{array}{c|c}
\mathbf{B} & 0 \\
\hline 0 & \mathrm{Id}_{8}
\end{array}\right)+O\left(\mu^{3 \kappa-1}\right) .
$$

Finally, when we use the coordinates $\left(q_{-}, p_{-}\right)$instead of $\left(\Theta_{-}, p_{-}\right)$, as we did in Substep 2.4, it follows from (10.28) that we get

$$
\frac{\partial\left(q_{-}, p_{-}, \mathbf{q}, \mathbf{p}\right)^{+}}{\partial\left(q_{-}, p_{-}, \mathbf{q}, \mathbf{p}\right)^{-}}=\frac{1}{\mu} O\left(\mu_{1 \times 2}^{\kappa}, 1_{1 \times 2} ; \mu_{1 \times 8}^{\kappa}\right) \otimes O\left(1_{1 \times 2}, \mu_{1 \times 2}^{\kappa} ; 0_{1 \times 8}\right)+O(1) .
$$

This is the structure of $d \mathbb{L}$ stated in the lemma.

It remains to obtain explicit asymptotics of the leading terms in Lemma 3.1. Below, we use the Delaunay variables $\left(L_{3}, \ell_{3}, G_{3}, g_{3} ; x_{1}, v_{1} ; G_{4}, g_{4}\right)^{ \pm}$as the orbit parameters outside the circle $\left|q_{-}\right|=2 \mu^{\kappa}$, and add a subscript "in" to the Delaunay variables inside the circle. We relate $C^{0}$ estimates of Lemma 10.2 to the $C^{1}$ estimates obtained above. Namely, consider the following equation which is obtained by discarding the $o(1)$ errors in (10.3):

$$
q_{-}^{+}=0, \quad p_{-}^{+}=R(\alpha) p_{-}^{-}, \quad \mathbf{q}^{+}=\mathbf{q}^{-} \quad \text { and } \quad \mathbf{p}^{+}=\mathbf{p}^{-},
$$

where $\alpha$ is given by (10.4). We have the following corollary saying that $d \mathbb{L}$ can be obtained by taking the derivative directly in (10.34). 
COROLlary 10.4. The vectors $\hat{u}_{j}$ and $\hat{\mathbf{l}}_{j}$ in Lemma 3.1 can be computed directly from (10.3) evaluated at the $j$-th Gerver collision point $j=1,2$ as follows:

$$
\begin{aligned}
& \hat{u}_{j}=\frac{\partial \mathcal{V}^{+}}{\partial \alpha} h=\frac{\partial \mathcal{V}^{+}}{\partial \mathcal{X}^{+}} \frac{\partial \mathcal{X}^{+}}{\partial\left(q_{-}, p_{-}, \mathbf{q}, \mathbf{p}\right)^{+}} \frac{\partial\left(q_{-}, p_{-}, \mathbf{q}, \mathbf{p}\right)^{+}}{\partial \alpha} h \\
& \hat{\mathbf{l}}_{j}=\frac{\partial G_{\mathrm{in}}}{\partial \mathcal{V}^{-}}=\frac{\partial G_{\mathrm{in}}}{\partial\left(q_{-}, p_{-}, \mathbf{q}, \mathbf{p}\right)^{-}} \frac{\partial\left(q_{-}, p_{-}, \mathbf{q}, \mathbf{p}\right)^{-}}{\partial \mathcal{X}^{-}} \frac{\partial \mathcal{X}^{-}}{\partial \mathcal{V}^{-}}
\end{aligned}
$$

where

$$
h=\lim _{\mu \rightarrow 0} \mu \frac{\partial \alpha}{\partial G_{\text {in }}}=2\left|v_{3}-v_{4}\right| \sin ^{2}\left(\frac{\alpha}{2}\right) .
$$

Here, $v_{3}$ and $v_{4}$ are the velocities of bodies 3 and 4 at the $j$-th Gerver collision point (see Appendix B), where $\left|v_{3}-v_{4}\right|$ is the same before and after the elastic collision. See Notation 2.3 for the use of $\mathcal{V}$ and $\mathcal{X}$.

Proof. We begin by computing the rank-1 terms in the expression for $\mathcal{D}$. To get (10.35), we need to multiply the vector by

$$
\frac{\partial\left(L_{3}, \ell_{3}, G_{3}, g_{3} ; q_{1}, p_{1} ; G_{4}, g_{4}\right)^{+}}{\partial\left(q_{3}, p_{3} ; q_{1}, p_{1} ; q_{4}, p_{4}\right)^{+}} \frac{\partial\left(q_{3}, p_{3} ; q_{1}, p_{1} ; q_{4}, p_{4}\right)^{+}}{\partial\left(q_{-}, p_{-}, \mathbf{q}, \mathbf{p}\right)^{+}}
$$

and the linear functional by

$$
\frac{\partial\left(q_{-}, p_{-}, \mathbf{q}, \mathbf{p}\right)^{-}}{\partial\left(q_{3}, p_{3} ; q_{1}, p_{1} ; q_{4}, p_{4}\right)^{-}} \frac{\partial\left(q_{3}, p_{3} ; q_{1}, p_{1} ; q_{4}, p_{4}\right)^{-}}{\partial\left(L_{3}, \ell_{3}, G_{3}, g_{3} ; q_{1}, p_{1} ; G_{4}, g_{4}\right)^{-}} .
$$

For the map (10.34), we have

$$
\frac{\partial(\mathbf{q}, \mathbf{p})^{+}}{\partial(\mathbf{q}, \mathbf{p})^{-}}=\operatorname{Id}_{8}, \quad \frac{\partial(\mathbf{q}, \mathbf{p})^{+}}{\partial\left(q_{-}, p_{-}\right)^{-}}=\frac{\partial\left(q_{-}, p_{-}\right)^{+}}{\partial(\mathbf{q}, \mathbf{p})^{-}}=0 \quad \text { and } \quad \frac{\partial(\mathbf{q}, \mathbf{p})^{+}}{\partial \alpha}=\frac{\partial G_{\text {in }}}{\partial(\mathbf{q}, \mathbf{p})^{-}}=0
$$

which agrees with the corresponding blocks in (10.33), up to an $o(1)$ error as $\mu \rightarrow 0$.

It remains to compare

$$
\frac{\partial\left(q_{-}, p_{-}\right)^{+}}{\partial\left(q_{-}, p_{-}\right)^{-}}
$$

Now, the expression for $\mathbf{l}_{j}$ follows from (10.30). Differentiating (10.34) we get

$$
\frac{\partial\left(q_{-}, p_{-}\right)^{+}}{\partial \alpha}=\left(0, \frac{\partial p_{-}^{+}}{\partial \alpha}\right)
$$

Thus, to get the expression of $\widehat{\mathbf{u}}$ in (10.35), it is enough to show (cf. (10.26)) that, for the map (10.34), we have

$$
\frac{\partial p_{-}^{+}}{\partial \alpha}\left(\frac{\partial \alpha}{\partial G_{\mathrm{in}}}\right)=\left(\frac{\partial p_{-}^{+}}{\partial G^{+}}+\frac{\partial \arctan \left(G^{-} / \mu \mathcal{L}^{-}\right)}{\partial G^{-}} \frac{\partial p_{-}^{+}}{\partial g^{+}}\right), \quad G_{\mathrm{in}}=G^{-} .
$$


Write $p_{-}^{+}=\mathbb{V}\left(G^{+}, \mu \mathcal{L}, g^{+}\right)$, where $G^{+}$and $g^{+}$depend on $G^{-}$as follows. First, $G^{+}=G^{-}$. Second, (A.3) gives

$$
\arctan \left(\frac{p_{2}^{ \pm}}{p_{1}^{ \pm}}\right) \sim g^{ \pm}-\arctan \left(\frac{G^{ \pm}}{\mu \mathcal{L}}\right) \quad \text { and } \arctan \left(\frac{p_{2}^{+}}{p_{1}^{+}}\right) \sim \arctan \left(\frac{p_{2}^{-}}{p_{1}^{-}}\right)+\alpha,
$$

where $\sim$ means that the difference between the left- and the right-hand side is $O\left(e^{-2 u}\right)$. Thus, $g^{+} \sim g^{-}+\alpha$, and so

$$
\frac{\partial p_{-}^{+}}{\partial G^{-}}=\frac{\partial \mathbb{V}}{\partial G^{+}}+\frac{\partial \mathbb{V}}{\partial g^{+}} \frac{\partial g^{+}}{\partial G^{-}} \sim \frac{\partial \mathbb{V}}{\partial G^{+}}+\frac{\partial \mathbb{V}}{\partial g^{+}} \frac{\partial \alpha}{\partial G^{-}},
$$

proving (10.36).

The next corollary says that the small remainders in (10.3) are also $C^{1}$ small, if the derivative is taken along the direction with small change of $G_{\text {in }}^{-}$.

Corollary 10.5. Let $\gamma(s):(-\varepsilon, \varepsilon) \rightarrow \mathbb{R}^{10}$ be a $C^{1}$ curve such that

$$
\Gamma=\gamma^{\prime}(0), \quad\|\Gamma\|=1 \quad \text { and } \quad \frac{d\left(G_{\mathrm{in}}^{-} \circ \gamma\right)(0)}{d s}=d G_{\mathrm{in}}^{-} \cdot \Gamma=O(\mu) .
$$

Then, when taking the derivative with respect to $s$ in the equations

$$
\left\{\begin{array}{l}
\left|p_{3}^{+}\right|^{2}+\left|p_{4}^{+}\right|^{2}=\left|p_{3}^{-}\right|^{2}+\left|p_{4}^{-}\right|^{2}+o(1) \\
\mathbf{p}^{+}=\mathbf{p}^{-}+o(1) \\
\mathbf{q}^{+}=\mathbf{q}^{-}+o(1)
\end{array}\right.
$$

obtained from equation (10.3), the error terms are also o(1) as $\mu \rightarrow 0$, after taking the directional derivative along the direction $\Gamma$.

Proof. For the motion of the mass center, it follows from Corollary 10.4 and (10.33) that

$$
\frac{\partial(\mathbf{q}, \mathbf{p})^{+}}{\partial\left(q_{-}, p_{-}, \mathbf{q}, \mathbf{p}\right)^{-}}=\frac{1}{\mu} \frac{\partial(\mathbf{q}, \mathbf{p})^{+}}{\partial \alpha} \otimes \tilde{\mathbf{l}}+\left(0_{8 \times 4}, \mathrm{Id}_{8}\right)+o(1), \quad \text { where } \tilde{\mathbf{l}}=\frac{\partial \alpha}{\partial G_{\mathrm{in}}^{-}} \frac{\partial G_{\mathrm{in}}^{-}}{\partial\left(q_{-}, p_{-}, \mathbf{q}, \mathbf{p}\right)^{-}}
$$

Here, $\partial \alpha / \partial G_{\text {in }}^{-}=O(1)$. We already obtained in equation (10.33) that $\partial(\mathbf{q}, \mathbf{p})^{+} / \partial \alpha=$ $O\left(\mu^{\kappa}\right)$, so our assumption

$$
\frac{d\left(G_{\mathrm{in}}^{-} \circ \gamma\right)(0)}{d s}=d G_{\mathrm{in}}^{-} \cdot \Gamma=O(\mu)
$$

implies that

$$
\tilde{\mathbf{l}} \frac{\partial\left(q_{-}, p_{-}, \mathbf{q}, \mathbf{p}\right)^{-}}{\partial \mathcal{V}^{-}} \cdot \Gamma=O(1) d G_{\mathrm{in}}^{-} \cdot \Gamma=O(\mu)
$$


which suppresses the $1 / \mu$ term. Here,

$$
\frac{\partial\left(q_{-}, p_{-}, \mathbf{q}, \mathbf{p}\right)^{-}}{\partial \mathcal{V}^{-}}=O(1)
$$

by Lemma A.3. This proves the last two identities of the corollary.

To derive the first equation, it is enough to show that

$$
\frac{d}{d s}\left(\left|p_{-}^{+}\right|^{2}-\left|p_{-}^{-}\right|^{2}\right)=o(1)
$$

since we already have the required estimate for the velocity of the center of mass. We use the fact that the right-hand side of (10.2) is the same in incoming and outgoing variables (superscripts + and - , respectively) by the total energy conservation. In (10.2), the terms involving only $\mathbf{q}$ and $\mathbf{p}$ are handled using the result of the previous paragraph. The term $-\mu /\left|q_{-}\right|$vanishes when taking the derivative since $\left|q_{-}\right|=\frac{1}{2} \mu^{\kappa}$ is constant. All the remaining terms have $q_{-}$to the power 2 or higher. We have $d q_{-}^{-} / d s=O(1)$, due to $\|\Gamma\|=1$, and $d q_{-}^{+} / d s=O(1)$, due to (10.37). Therefore, after taking the derivative with respect to $s$, any term involving $q_{-}$is of order $O\left(\mu^{\kappa}\right)$. This completes the proof of the energy conservation part.

\subsection{Proof of Lemma $3.4(\mathrm{c})$}

In this section, we work out the $O(1 / \mu)$ term in the local map and prove Lemma 3.4 (c).

Proof. • Before collision, $\hat{\mathbf{l}}=\nabla_{\mathcal{V}^{-}} G_{\text {in }}^{-}$.

In this calculation, every variable should carry a superscript "_", and we omit it for simplicity. To verify $\hat{\mathbf{l}}_{i} \cdot w_{3-i} \neq 0$ and $\hat{\mathbf{l}}_{i} \cdot \widetilde{w} \neq 0$ for $i=1,2$ in Lemma 3.4 (c), noting that $\widetilde{w}=\left(0,1,0_{1 \times 8}\right)$ and $w=\left(0_{1 \times 8}, *, *\right)$, it is enough to work out the three entries

$$
\nabla_{\ell_{3}} G_{\mathrm{in}}, \quad \nabla_{G_{4}} G_{\mathrm{in}} \text { and } \quad \nabla_{g_{4}} G_{\mathrm{in}}
$$

in $\hat{\mathbf{l}}$. According to Corollary 10.4, we can differentiate the asymptotic expression of Lemma 10.2. We have

$$
\begin{aligned}
& \left(\nabla_{G_{4}} G_{\mathrm{in}}, \nabla_{g_{4}} G_{\mathrm{in}}\right) \\
& \quad=-\left(p_{3}-p_{4}\right) \times\left(\frac{\partial}{\partial G_{4}}, \frac{\partial}{\partial g_{4}}\right) q_{4}-\left(p_{3}-p_{4}\right) \times\left(\frac{\partial q_{4}}{\partial \ell_{4}}\right) \cdot\left(\nabla_{G_{4}} \ell_{4}, \nabla_{g_{4}} \ell_{4}\right)+O\left(\mu^{\kappa}\right),
\end{aligned}
$$

where $O\left(\mu^{\kappa}\right)$ comes from

$$
\left(\left(\frac{\partial}{\partial G_{4}}, \frac{\partial}{\partial g_{4}}\right)\left(p_{3}-p_{4}\right)\right) \times\left(q_{3}-q_{4}\right)
$$


and dominates

$$
\frac{\partial q_{4}}{\partial L_{4}} \cdot \nabla_{G_{4}, g_{4}} L_{4}=O(\mu)
$$

Indeed, we have $\partial q_{4} / \partial L_{4}=O(1)$ and

$$
\frac{\mu}{\left|x_{3} /(1+\mu)-x_{4}\right|}=\frac{\mu}{\left|q_{3}-q_{4}\right|}=\mu^{1-\kappa},
$$

and using (4.8) with $H=0$, the leading contribution to $\nabla_{G_{4}, g_{4}} L_{4}$ is given by

$$
\nabla_{G_{4}, g_{4}}\left(\frac{1}{\left|x_{4}\right|}-\frac{1}{\left|x_{4}+\mu x_{3} /(1+\mu)\right|}\right)=O(\mu)
$$

where we use Newton-Leibniz

$$
\frac{1}{\left|x_{4}\right|}-\frac{1}{\left|x_{4}+\mu x_{3} /(1+\mu)\right|}=\mu \int_{0}^{1} \frac{x_{4}+t \mu x_{3} /(1+\mu)}{\left|x_{4}+t \mu x_{3} /(1+\mu)\right|^{3}} \cdot \frac{x_{3}}{1+\mu} d t
$$

and Lemma A.3 for $\nabla_{G_{4}, g_{4}} x_{4}=O(1)$.

We next eliminate $\ell_{4}$ using the relation $\left|q_{3}-q_{4}\right|=\mu^{\kappa}$ :

$$
\begin{aligned}
\left(\nabla_{G_{4}} \ell_{4}, \nabla_{g_{4}} \ell_{4}\right) & =-\left(\frac{\partial\left|q_{3}-q_{4}\right|}{\partial \ell_{4}}\right)^{-1}\left[\left(\frac{\partial\left|q_{3}-q_{4}\right|}{\partial G_{4}}, \frac{\partial\left|q_{3}-q_{4}\right|}{\partial g_{4}}\right)+\frac{\partial\left|q_{3}-q_{4}\right|}{\partial L_{4}}\left(\nabla_{G_{4}, g_{4}} L_{4}\right)\right] \\
& =-\frac{\left(q_{3}-q_{4}\right) \cdot\left(\partial q_{4} / \partial G_{4}, \partial q_{4} / \partial g_{4}\right)}{\left(q_{3}-q_{4}\right) \cdot \partial q_{4} / \partial \ell_{4}}+O(\mu) \\
& =-\frac{\left(p_{3}-p_{4}\right) \cdot\left(\partial q_{4} / \partial G_{4}, \partial q_{4} / \partial g_{4}\right)}{\left(p_{3}-p_{4}\right) \cdot \partial q_{4} / \partial \ell_{4}}+O\left(\mu^{1-\kappa}\right) .
\end{aligned}
$$

Here, we replaced $q_{3}-q_{4}$ by $p_{3}-p_{4}$, using the fact that the two vectors form an angle of order $O\left(\mu^{1-\kappa}\right)$ by Lemma $10.2(\mathrm{c})$. Therefore,

$$
\begin{aligned}
\left(\nabla_{G_{4}} G_{\text {in }}, \nabla_{g_{4}} G_{\text {in }}\right)=- & \left(p_{3}-p_{4}\right) \times\left(\frac{\partial}{\partial G_{4}}, \frac{\partial}{\partial g_{4}}\right) q_{4} \\
& +\left(p_{3}-p_{4}\right) \times \frac{\partial q_{4}}{\partial \ell_{4}}\left(\frac{\left(p_{3}-p_{4}\right) \cdot\left(\partial q_{4} / \partial G_{4}, \partial q_{4} / \partial g_{4}\right)}{\left(p_{3}-p_{4}\right) \cdot \partial q_{4} / \partial \ell_{4}}\right)+O\left(\mu^{\kappa}+\mu^{1-2 \kappa}\right) .
\end{aligned}
$$

Similarly, we get

$$
\nabla_{\ell_{3}} G_{\text {in }}=\left(p_{3}-p_{4}\right) \times \frac{\partial q_{3}}{\partial \ell_{3}}+\left(p_{3}-p_{4}\right) \times \frac{\partial q_{4}}{\partial \ell_{4}}\left(\frac{\left(p_{3}-p_{4}\right) \cdot \partial q_{3} / \partial \ell_{3}}{\left(p_{3}-p_{4}\right) \cdot \partial q_{4} / \partial \ell_{4}}\right)+O\left(\mu^{\kappa}+\mu^{1-2 \kappa}\right)
$$

We use the software MATHEMAтіCA to work out the three entries and check directly that $\hat{\mathbf{l}}_{i} \cdot w_{3-i} \neq 0$ and $\hat{\mathbf{l}}_{i} \cdot \widetilde{w} \neq 0$ for $i=1,2$ using Lemma 3.2 . 
- After collision, $\widehat{\mathbf{u}}=\partial \mathcal{V}^{+} / \partial \alpha$.

In equation (10.3), we let $\mu \rightarrow 0$. Applying the implicit function theorem to (10.3) with $\mu=0$, we obtain

$$
\begin{aligned}
\frac{\partial}{\partial \alpha} & \left(q_{3}^{+}, p_{3}^{+} ; q_{1}^{+}, p_{1}^{+} ; q_{4}^{+}, p_{4}^{+}\right) \\
& =\frac{1}{2}\left(0,0, R\left(\frac{\pi}{2}+\alpha\right)\left(p_{3}^{-}-p_{4}^{-}\right) ; 0,0,0,0 ; 0,0,-R\left(\frac{\pi}{2}+\alpha\right)\left(p_{3}^{-}-p_{4}^{-}\right)\right)^{T} \\
& =\frac{1}{2}\left(0,0, R\left(\frac{\pi}{2}\right)\left(p_{3}^{+}-p_{4}^{+}\right) ; 0,0,0,0 ; 0,0,-R\left(\frac{\pi}{2}\right)\left(p_{3}^{+}-p_{4}^{+}\right)\right)^{T},
\end{aligned}
$$

where

$$
R\left(\frac{\pi}{2}+\alpha\right)=\frac{d R(\alpha)}{d \alpha}
$$

and $\nabla_{\mathcal{V}} \ell_{4}^{+}$is given by (10.13). Again, we use Mathematica to work out $\partial \mathcal{V}^{+} / \partial \alpha$, and check directly that $\overline{\mathbf{l}}_{i} \cdot \widehat{\mathbf{u}}_{i} \neq 0$ for $i=1,2$, using Lemma 3.2.

To obtain a symbolic sequence with any order of symbols 3 and 4 as claimed in the main theorem, we notice that the only difference is that the outgoing relative velocity changes sign $\left(p_{3}^{+}-p_{4}^{+}\right) \mapsto-\left(p_{3}^{+}-p_{4}^{+}\right)$. So, we only need to send $\widehat{\mathbf{u}} \mapsto-\widehat{\mathbf{u}}$.

\subsection{Proof of Lemma $3.4(a)$ and (b)}

In this section, we prove Lemma 3.4 (a) and (b). Since we have already obtained $\mathbf{l}$ and $\mathbf{u}$ in $d \mathbb{L}$ and $\overline{\mathbf{l}}, \overline{\overline{\mathbf{l}}}, \overline{\mathbf{u}}$ and $\overline{\overline{\mathbf{u}}}$ in $d \mathbb{G}$, one way to prove Lemma 3.4 is to work out the matrix $B$ explicitly using an argument similar to that in Corollary 10.4. In that case, the current section is not necessary. However, in this section, we use a different approach, which simplifies the computation and has clear physical and geometrical meaning. We first abbreviate $d \mathbb{L}$ in Lemma 3.1 (a) as

$$
d \mathbb{L}(\boldsymbol{x})=\frac{1}{\mu} \mathbf{u}_{j, \mu} \otimes \mathbf{l}_{j, \mu}+B_{j, \mu},
$$

using the subscript $\mu$ to absorb the $\mu$-depending $o(1)$ 's. Similarly, we write

$$
d \mathbb{G}=\chi^{2} \overline{\mathbf{u}}_{j, \mu} \otimes \overline{\mathbf{l}}_{j, \mu}+4 \chi \overline{\mathbf{\mathbf { u }}}_{j, \mu} \otimes \overline{\overline{\mathbf{l}}}_{j, \mu}+O(\mu \chi) .
$$

Lemma 10.6. Consider $\boldsymbol{x}_{\mu} \in U_{j}(\delta), j=1,2$, and $\left|\bar{\theta}_{4}^{+}-\pi\right|<\tilde{\theta}$ as in Lemma 3.1. Suppose the vector

$$
\widetilde{\Gamma}_{j, \mu} \in \operatorname{span}\left\{\overline{\mathbf{u}}_{3-j}, \overline{\overline{\mathbf{u}}}_{3-j}\right\} \subset T_{\boldsymbol{x}_{\mu}} U_{j}(\delta)
$$


for $j=2$, and

$$
\widetilde{\Gamma}_{j, \mu} \in \operatorname{span}\left\{d \mathcal{R} \overline{\mathbf{u}}_{3-j}, d \mathcal{R} \overline{\overline{\mathbf{u}}}_{3-j}\right\} \subset T_{\boldsymbol{x}_{\mu}} U_{j}(\delta)
$$

for $j=1$, satisfies

$$
\overline{\mathbf{l}}_{j}\left(d \mathbb{L} \widetilde{\Gamma}_{j, \mu}\right)=0 \quad \text { and } \quad\left\|\widetilde{\Gamma}_{j, \mu}\right\|_{\infty}=1 .
$$

Then, we have

(a) $\mathbf{l}_{j, \mu}\left(\widetilde{\Gamma}_{j, \mu}\right)=O(\mu)$ as $\mu \rightarrow 0$;

(b) the limits $\lim _{\mu \rightarrow 0} \widetilde{\Gamma}_{j, \mu}$ and $\lim _{\mu \rightarrow 0} d \mathbb{L} \widetilde{\Gamma}_{j, \mu}$ exist, and $\lim _{\mu \rightarrow 0} \widetilde{\Gamma}_{j, \mu}$ is continuous in $\widehat{\boldsymbol{x}}$ (see Lemma 3.1) and $\lim _{\mu \rightarrow 0} d \mathbb{L} \widetilde{\Gamma}_{j, \mu}$ is continuous in $\widehat{\boldsymbol{x}}$ and $\bar{\theta}_{4}^{+}$;

(c) $\hat{\overline{\mathbf{l}}}_{j}\left(\lim _{\delta, \tilde{\theta} \rightarrow 0} \lim _{\mu \rightarrow 0} d \mathbb{L} \widetilde{\Gamma}_{j, \mu}\right)=0$.

Proof. For simplicity, we give the proof in the case $j=2$ without needing the renormalization. The other case $j=1$ is completely analogous. Denote

$$
\Gamma_{2, \mu}^{\prime}=\mathbf{l}_{2, \mu}\left(\overline{\mathbf{u}}_{1, \mu}\right) \overline{\overline{\mathbf{u}}}_{1, \mu}-\mathbf{l}_{2, \mu}\left(\overline{\overline{\mathbf{u}}}_{1, \mu}\right) \overline{\mathbf{u}}_{1, \mu} \in \operatorname{Ker} \mathbf{l}_{2, \mu},
$$

and let $v_{\mu}$ be a vector in $\operatorname{span}\left(\overline{\mathbf{u}}_{1}, \overline{\mathbf{u}}_{1}\right)$ such that $v_{\mu} \rightarrow v$ as $\mu \rightarrow 0$ and $\mathbf{l}_{2, \mu}\left(v_{\mu}\right)=1$. Suppose that $\widetilde{\Gamma}_{2, \mu}=a_{\mu} v_{\mu}+b_{\mu} \Gamma_{2, \mu}^{\prime}$. Then

$$
d \mathbb{L}\left(\widetilde{\Gamma}_{2, \mu}\right)=\frac{a_{\mu}}{\mu} \mathbf{l}_{2, \mu}\left(v_{\mu}\right) \mathbf{u}_{2, \mu}+a_{\mu} B_{2, \mu}\left(v_{\mu}\right)+b_{\mu} B_{2, \mu} \Gamma_{2, \mu}^{\prime} .
$$

So, $\overline{\mathbf{l}}_{2}\left(d \mathbb{L}\left(\widetilde{\Gamma}_{2, \mu}\right)\right)=0$ implies that

$$
a_{\mu}=-\mu \frac{b_{\mu} \overline{\mathbf{l}}_{2}\left(B_{2, \mu} \Gamma_{2, \mu}^{\prime}\right)}{\mathbf{l}_{2, \mu}\left(v_{\mu}\right) \overline{\mathbf{l}}_{2}\left(\mathbf{u}_{2, \mu}\right)+\mu \overline{\mathbf{l}}_{2} B_{2, \mu}\left(v_{\mu}\right)} .
$$

The denominator is not zero, since $\mathbf{l}_{2, \mu}\left(v_{\mu}\right)=1$ and $\overline{\mathbf{l}}_{2}\left(\mathbf{u}_{2, \mu}\right) \neq 0$ using Lemma 3.4 (c). Therefore, $a_{\mu}=O(\mu)$ and $b_{\mu}=O(1)$ using $\left\|\Gamma_{2, \mu}\right\|_{\infty}=1$. Hence, $\widetilde{\Gamma}_{2, \mu}=b_{\mu} \Gamma_{2, \mu}^{\prime}+O(\mu)$ and $\mathbf{l}_{2, \mu}\left(\widetilde{\Gamma}_{2, \mu}\right)=O(\mu)$. The continuous dependence on variables in part (b) follows from part (a) of Lemmas 3.1 and 3.2. Now, the remaining statements of the lemma follow from equations (10.38) and (10.39).

To check the non-degeneracy condition, it is enough to know the following.

LeMmA 10.7. Let $\boldsymbol{x}_{\mu} \in U_{j}(\delta)$ and $\left|\bar{\theta}_{4}^{+}-\pi\right|<\tilde{\theta} \ll 1$ be as in Lemma 3.1. If we take the directional derivative at $\boldsymbol{x}_{\mu}$ of the local map along a direction

$$
\Gamma_{j, \mu} \in \operatorname{span}\left\{\overline{\mathbf{u}}_{3-j}, \overline{\overline{\mathbf{u}}}_{3-j}\right\} \subset T_{\boldsymbol{x}_{\mu}} U_{j}(\delta)
$$

for $j=2$ and

$$
\Gamma_{j, \mu} \in \operatorname{span}\left\{d \mathcal{R} \overline{\mathbf{u}}_{3-j}, d \mathcal{R} \overline{\overline{\mathbf{u}}}_{3-j}\right\} \subset T_{\boldsymbol{x}_{\mu}} U_{j}(\delta)
$$


for $j=1$, such that $\overline{\mathbf{l}}_{j} \cdot\left(d \mathbb{L} \Gamma_{j, \mu}\right)=0, j=1,2$, then

$$
\lim _{1 / \chi \ll \mu \rightarrow 0} \frac{\partial E_{3}^{+}}{\partial \Gamma_{j, \mu}}
$$

is a continuous function of both $\boldsymbol{x}$ and $\bar{\theta}_{4}^{+}$, where $E_{3}^{+}$(resp. $\left.\bar{\theta}_{4}^{+}\right)$is the energy of $Q_{3}$ (resp. the outgoing asymptote of $Q_{4}$ ) after the close encounter with $Q_{4}$. If we take further limits $\delta \rightarrow 0$ and $\tilde{\theta} \rightarrow 0$, we have

$$
\lim _{\delta, \tilde{\theta} \rightarrow 0} \lim _{1 / \chi \ll \mu \rightarrow 0} \frac{\partial E_{3}^{+}}{\partial \Gamma_{j, \mu}} \neq 0, \quad j=1,2 .
$$

Now, we can check the non-degeneracy condition.

Proof of Lemma 3.4 (a) and (b). We prove (b1) and (b2). The proofs of (a1) and (a2) are similar and are left to the reader. To check (b2), de 4 , we differentiate

$$
e_{4}=\sqrt{1+\left(\frac{G_{4}}{L_{4}}\right)^{2}}
$$

to get

$$
d e_{4}=\frac{1}{e_{4}}\left(\frac{G_{4}}{L_{4}^{2}} d G_{4}-\frac{G_{4}^{2}}{L_{4}^{3}} d L_{4}\right) .
$$

Thus, Lemma 3.2 gives $d e_{4} w=G_{4} / L_{4}^{2} \neq 0$ as claimed.

Next, we check (b1) which is equivalent to the following condition

$$
\operatorname{det}\left(\begin{array}{ll}
\hat{\overline{\mathbf{l}}}_{2}\left(\widehat{\mathbf{u}}_{2}\right) & \hat{\overline{\mathbf{l}}}_{2}\left(\widehat{B}_{2} \Gamma_{2}^{\prime}\right) \\
\hat{\overline{\mathbf{l}}}_{2}\left(\widehat{\mathbf{u}}_{2}\right) & \hat{\overline{\mathbf{l}}}_{2}\left(\widehat{B}_{2} \Gamma_{2}^{\prime}\right)
\end{array}\right) \neq 0
$$

where $\Gamma_{2}^{\prime}=\hat{\mathbf{l}}_{2}(\widetilde{w}) w_{1}-\hat{\mathbf{l}}_{2}\left(w_{1}\right) \widetilde{w}$. We have $\Gamma_{2}^{\prime} \neq 0$, due to Lemma $3.4(\mathrm{c})$.

Let $\Gamma_{2}$ be a vector satisfying $\hat{\overline{\mathbf{l}}}_{2} \cdot\left(d \mathbb{L} \Gamma_{2}\right)=0$ and chosen as follows: $d \mathbb{L} \Gamma_{2}$ is a vector in $\operatorname{span}\left\{\widehat{\mathbf{u}}_{2}, \widehat{B}_{2} \Gamma_{2}^{\prime}\right\}$, so it can be represented as $d \mathbb{L} \Gamma_{2}=b \widehat{\mathbf{u}}_{2}+b^{\prime} \widehat{B}_{2} \Gamma_{2}^{\prime}$. Thus, we can take $b=-\hat{\overline{\mathbf{l}}}_{2} \cdot \widehat{B}_{2} \Gamma_{2}^{\prime}$ and $b^{\prime}=\hat{\overline{\mathbf{l}}}_{2}\left(\widehat{\mathbf{u}}_{2}\right)$ to ensure that $d \mathbb{L} \Gamma_{2} \in \operatorname{Ker} \hat{\overline{\mathbf{l}}}_{2}$. Note that we have $b^{\prime} \neq 0$ by Lemma $3.4(\mathrm{c})$. Hence,

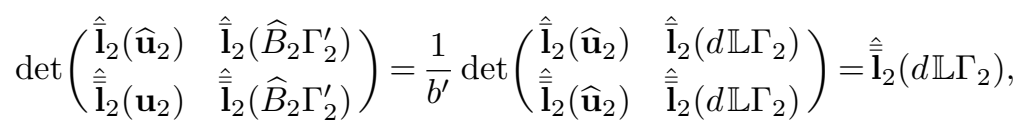

where the last equality holds since $\hat{\overline{\mathbf{l}}}_{2}\left(d \mathbb{L} \Gamma_{2}\right)=0$. By Lemma $3.2, \hat{\overline{\mathbf{l}}}_{i}=\left(1,0_{1 \times 9}\right)$. Therefore,

$$
\hat{\overline{\bar{l}}}_{2}\left(d \mathbb{L} \Gamma_{2}\right)=\frac{\partial E_{3}^{+}}{\partial \Gamma_{2}}
$$

and so (b2) follows from Lemma 10.7. 
It remains to prove Lemma 10.7. It is more convenient for us to work with polar coordinates. We need the following quantities.

Definition 10.8. We denote by $\psi$ the polar angle, related to $u$ by

$$
\tan \left(\frac{\psi}{2}\right)=\sqrt{\frac{1+e}{1-e}} \tan \left(\frac{u}{2}\right)
$$

for an ellipse. We choose the positive $y$ axis as the axis $\psi=0$. We let $E$ denote the energy, $e$ the eccentricity, $G$ the angular momentum and $g$ the argument of apapsis.

Recall the formula

$$
r=\frac{G^{2}}{1-e \cos \psi}
$$

for conic sections in which the periapsis lies on the axis $\psi=\pi$. In our case, we have, as $1 / \chi \ll \mu \rightarrow 0$,

$$
\left\{\begin{array}{l}
r_{3}^{ \pm}=\frac{\left(G_{3}^{ \pm}\right)^{2}}{1-e_{3}^{ \pm} \sin \left(\psi_{3}^{ \pm}+g_{3}^{ \pm}\right)}+o(1), \\
r_{4}^{ \pm}=\frac{\left(G_{4}^{ \pm}\right)^{2}}{1-e_{4}^{ \pm} \sin \left(\psi_{4}^{ \pm}-g_{4}^{ \pm}\right)}+o(1)
\end{array}\right.
$$

LEMMA 10.9. Under the assumptions of Corollary 10.5, we have

$$
\frac{d r_{3}^{+}}{d s}=\frac{d r_{4}^{+}}{d s}+o(1), \quad \frac{d r_{3}^{-}}{d s}=\frac{d r_{4}^{-}}{d s}+o(1), \quad \frac{d \psi_{3}^{+}}{d s}=\frac{d \psi_{4}^{+}}{d s}+o(1), \quad \frac{d \psi_{3}^{-}}{d s}=\frac{d \psi_{4}^{-}}{d s}+o(1)
$$

Moreover, in (10.41) the o(1) terms are also $C^{1}$ small when taking the derivative with respect to $s$.

Proof. To prove the statement about (10.41), we use the Hamiltonian (4.8). We have seen in the beginning of the proof of Lemma 10.3 that $-\mu /\left|q_{3}-q_{4}\right|$ gives an $O\left(\mu^{1-2 \kappa}\right)$ perturbation to the variational equations. This shows that the perturbation to the Kepler motion is $C^{1}$ small.

Next, we consider the derivatives $d r_{3,4}^{ \pm} / d s$. We consider first the case of "-". From the condition $\left|\vec{r}_{3}-\vec{r}_{4}\right|=\mu^{\kappa}$, for the Poincaré section we get

$$
\left(\vec{r}_{3}-\vec{r}_{4}\right) \cdot \frac{d}{d s}\left(\vec{r}_{3}-\vec{r}_{4}\right)=0
$$

and hence

$$
\left(\vec{r}_{3}-\vec{r}_{4}\right) \perp \frac{d}{d s}\left(\vec{r}_{3}-\vec{r}_{4}\right) .
$$

We also know the angular momentum for the relative motion is

$$
G_{\text {in }}=\left(\dot{\vec{r}}_{3}-\dot{\vec{r}}_{4}\right) \times\left(\vec{r}_{3}-\vec{r}_{4}\right)=O(\mu)
$$


which implies that $\dot{\vec{r}}_{3}-\dot{\vec{r}}_{4}$ is almost parallel to $\vec{r}_{3}-\vec{r}_{4}$, by Lemma $10.2(\mathrm{c})$, and hence $d\left(\vec{r}_{3}-\vec{r}_{4}\right) / d s$ is almost perpendicular to $\dot{\vec{r}}_{3}-\dot{\vec{r}}_{4}$. The condition

$$
\frac{d G_{\mathrm{in}}^{-}}{d s}=O(\mu)
$$

reads

$$
\left(\frac{d}{d s}\left(\dot{\vec{r}}_{3}-\dot{\vec{r}}_{4}\right)\right) \times\left(\vec{r}_{3}-\vec{r}_{4}\right)+\left(\dot{\vec{r}}_{3}-\dot{\vec{r}}_{4}\right) \times\left(\frac{d}{d s}\left(\vec{r}_{3}-\vec{r}_{4}\right)\right)=O(\mu) .
$$

Since the first term is $O\left(\mu^{\kappa}\right)$ due to our choice of the Poincaré section, we see that

$$
\left(\dot{\vec{r}}_{3}-\dot{\vec{r}}_{4}\right) \times\left(\frac{d}{d s}\left(\vec{r}_{3}-\vec{r}_{4}\right)\right)=o(1) .
$$

Since $d\left(\vec{r}_{3}-\vec{r}_{4}\right) / d s$ is almost perpendicular to $\left(\dot{\vec{r}}_{3}-\dot{\vec{r}}_{4}\right)$ by the analysis above, we get

$$
\frac{d}{d s}\left(\vec{r}_{3}-\vec{r}_{4}\right)=o(1)
$$

Taking the radial and angular part of this vector identity, and using that $r_{4}=r_{3}+o(1)$ and $\psi_{4}=\psi_{3}+o(1)$, we get the "-" part of the lemma.

To repeat the above argument for "+" variables, we first need to establish that $d G_{\text {in }}^{+} / d s=O(\mu)$. Indeed, using equations (10.19) and (10.29), we get

$$
\begin{aligned}
\frac{\partial G_{\text {in }}^{+}}{\partial s} & =\frac{\partial G_{\text {in }}^{+}}{\partial\left(\mathcal{L}, G_{\text {in }}, g, \mathbf{q}, \mathbf{p}\right)^{-}} \frac{\partial\left(\mathcal{L}, G_{\text {in }}, g, \mathbf{q}, \mathbf{p}\right)^{-}}{\partial s} \\
& =O\left(\mu^{3 \kappa}, 1, \mu^{3 \kappa}, \mu_{1 \times 4}^{3 \kappa}, \mu_{1 \times 4}^{3 \kappa}\right) \cdot O\left(1, \mu, 1,1_{1 \times 4}, 1_{1 \times 4}\right)=O(\mu) .
\end{aligned}
$$

It remains to show that $d\left(\dot{\vec{r}}_{3}-\dot{\vec{r}}_{4}\right) / d s=O(1)$ in the "+" case. Since we know it is true in the "-" case, the "+" case follows, because the directional derivative of the local map $d \mathbb{L} \Gamma$ is bounded, due to our choice of $\Gamma$.

We are now ready to describe the computation of Lemma 10.7. We will use the following set of equations which follows from (10.34):

$$
\begin{gathered}
E_{3}^{+}+E_{4}^{+}=E_{3}^{-}+E_{4}^{-}, \\
G_{3}^{+}+G_{4}^{+}=G_{3}^{-}+G_{4}^{-}, \\
\frac{e_{3}^{+}}{G_{3}^{+}} \cos \left(\psi_{3}^{+}+g_{3}^{+}\right)+\frac{e_{4}^{+}}{G_{4}^{+}} \cos \left(\psi_{4}^{-}-g_{4}^{-}\right)=\frac{e_{3}^{-}}{G_{3}^{-}} \cos \left(\psi_{3}^{-}+g_{3}^{-}\right)+\frac{e_{4}^{-}}{G_{4}^{-}} \cos \left(\psi_{4}^{-}-g_{4}^{-}\right), \\
\frac{\left(G_{3}^{+}\right)^{2}}{1-e_{3}^{+} \sin \left(\psi_{3}^{+}+g_{3}^{+}\right)}=\frac{\left(G_{3}^{-}\right)^{2}}{1-e_{3}^{-} \sin \left(\psi_{3}^{-}+g_{3}^{-}\right)},
\end{gathered}
$$




$$
\begin{gathered}
\frac{\left(G_{3}^{+}\right)^{2}}{1-e_{3}^{+} \sin \left(\psi_{3}^{+}+g_{3}^{+}\right)}=\frac{\left(G_{4}^{+}\right)^{2}}{1-e_{4}^{+} \sin \left(\psi_{4}^{+}-g_{4}^{+}\right)}, \\
\frac{\left(G_{3}^{-}\right)^{2}}{1-e_{3}^{-} \sin \left(\psi_{3}^{-}+g_{3}^{-}\right)}=\frac{\left(G_{4}^{-}\right)^{2}}{1-e_{4}^{-} \sin \left(\psi_{4}^{-}-g_{4}^{-}\right)}, \\
\psi_{3}^{+}=\psi_{3}^{-}, \quad \psi_{4}^{-}=\psi_{3}^{-}, \quad \psi_{4}^{+}=\psi_{3}^{+}, \quad x_{1}^{+}=x_{1}^{-}, \quad v_{1}^{+}=v_{1}^{-} .
\end{gathered}
$$

In the above equations, we have dropped $o(1)$ terms for brevity. We would like to emphasize that the above approximations hold not only in the $C^{0}$ sense but also in the $C^{1}$ sense when we take the derivatives along directions satisfying the conditions of Corollary 10.5. Equation (10.42) is the approximate conservation of energy, (10.43) is the approximate conservation of angular momentum and (10.44) follows from the approximate conservation of momentum as follows. Represent the position vector as $\vec{r}=r \hat{e}_{r}$. Then, the velocity is $\dot{\vec{r}}=\dot{r} \hat{e}_{r}+r \dot{\psi} \hat{e}_{\psi}$. Conservation of momentum gives

$$
\left(\dot{\vec{r}}_{3}\right)^{-}+\left(\dot{\vec{r}}_{4}\right)^{-}=\left(\dot{\vec{r}}_{3}\right)^{+}+\left(\dot{\vec{r}}_{4}\right)^{+} .
$$

Taking the radial component and using the polar representation of the ellipse

$$
r=\frac{G^{2}}{1-e \sin (\psi+g)},
$$

we get

$$
\dot{r}=\frac{G^{2}}{(1-e \sin (\psi+g))^{2}} e \cos (\psi+g) \dot{\psi}=\frac{r^{2}}{G^{2}} e \cos (\psi+g) \frac{G}{r^{2}}=\frac{e}{G} \cos (\psi+g) .
$$

The possibility of differentiating these equations is justified in Corollary 10.5. The remaining equations reflect the fact that $Q_{3}^{ \pm}$and $Q_{4}^{ \pm}$are all close to each other. The possibility of differentiating these equations is justified by Lemma 10.9. We set the total energy to be zero. So, we get $E_{4}^{ \pm}=-E_{3}^{ \pm}$. This eliminates $E_{4}^{ \pm}$. Then, we also eliminate $\psi_{4}^{ \pm}$by setting them equal to $\psi_{3}^{ \pm}$.

Proof of the Lemma 10.7. Lemma 10.6 and Corollary 10.4 show that the assumption of Lemma 10.7 implies that the direction $\Gamma$ along which we take the directional derivative satisfies $\partial G_{\text {in }} / \partial \Gamma=O(\mu)$. So, we can directly take derivatives in equations (10.42)(10.47). Recall that we need to compute $d E_{3}^{+}(d \mathbb{L} \Gamma)$, where $\Gamma \in \operatorname{Ker} \mathbf{l}_{j} \cap \operatorname{span}\left\{w_{3-j}, \widetilde{w}\right\}$. Lemma 3.2 tells us that in Delaunay coordinates we have

$$
\widetilde{w}=\left(0,1,0_{1 \times 8}\right) \quad \text { and } \quad w=\left(0_{1 \times 8}, 1, a\right) \text { where } a=-\frac{L_{4}^{-}}{\left(L_{4}^{-}\right)^{2}+\left(G_{4}^{-}\right)^{2}} .
$$

The formula

$$
\tan \left(\frac{\psi}{2}\right)=\sqrt{\frac{1+e}{1-e}} \tan \left(\frac{u}{2}\right)
$$


which relates $\psi$ to $\ell$ through $u$ shows that (10.49) also holds if we use

$$
\left(L_{3}, \psi_{3}, G_{3}, g_{3} ; x_{1}, v_{1} ; G_{4}, g_{4}\right)
$$

as coordinates. Hence, $\Gamma$ has the form $\left(0,1,0_{1 \times 6}, c, c a\right)$. To find the constant $c$, we use (10.47).

Using (10.48), we can replace $\psi_{3}^{+}=\psi_{3}^{-}=\psi_{4}^{+}=\psi_{4}^{-}$by $\psi$, and get rid of $x_{1}$ and $v_{1}$. Let $\boldsymbol{L}$ denote the projection of $\mathbb{L}$ to the variables $\left(E_{3}, G_{3}, g_{3}, G_{4}, g_{4}\right)$, and let $\boldsymbol{\Gamma}$ denote the projection of $\Gamma$ to the variables $\left(E_{3}, \psi, G_{3}, g_{3}, G_{4}, g_{4}\right)$. Thus, we need to find $d E_{3}^{+}(d \boldsymbol{L} \boldsymbol{\Gamma})$. To this end, we write the remaining equations (10.43)-(10.46) formally as $\mathbf{F}\left(Z^{+}, Z^{-}\right)=0$, where $Z^{+}=\left(E_{3}^{+}, G_{3}^{+}, g_{3}^{+}, G_{4}^{+}, g_{4}^{+}\right)$and $Z^{-}=\left(E_{3}^{-}, \psi, G_{3}^{-}, g_{3}^{-}, G_{4}^{-}, g_{4}^{-}\right)$. We have

$$
\frac{\partial \mathbf{F}}{\partial Z^{+}} d \boldsymbol{L} \boldsymbol{\Gamma}+\frac{\partial \mathbf{F}}{\partial Z^{-}} \boldsymbol{\Gamma}=0 .
$$

However, $\partial \mathbf{F} / \partial Z^{+}$is not invertible, since $\mathbf{F}$ involves only four equations while $Z^{+}$has five variables. To resolve this problem, we notice that, by definition of $\Gamma$, we have $\overline{\mathbf{l}} \cdot d \boldsymbol{L} \boldsymbol{\Gamma}=\mathbf{0}$, where

$$
\overline{\mathbf{l}}=\left(\frac{G_{4}^{+} / L_{4}^{+}}{\left(L_{4}^{+}\right)^{2}+\left(G_{4}^{+}\right)^{2}}, 0,0,-\frac{1}{\left(L_{4}^{+}\right)^{2}+\left(G_{4}^{+}\right)^{2}}, \frac{1}{L_{4}^{+}}\right),
$$

by Lemma 3.2. Thus, we get

$$
\left[\begin{array}{c}
\overline{\mathbf{l}} \\
\frac{\partial \mathbf{F}}{\partial Z^{+}}
\end{array}\right] d \boldsymbol{L} \boldsymbol{\Gamma}=-\left[\begin{array}{c}
0 \\
\frac{\partial \mathbf{F}}{\partial Z^{-}} \boldsymbol{\Gamma}
\end{array}\right] \text { and } d \boldsymbol{L} \boldsymbol{\Gamma}=-\left[\begin{array}{c}
\overline{\mathbf{l}} \\
\frac{\partial \mathbf{F}}{\partial Z^{+}}
\end{array}\right]^{-1}\left[\begin{array}{c}
0 \\
\frac{\partial \mathbf{F}}{\partial Z^{-}} \boldsymbol{\Gamma}
\end{array}\right] .
$$

We only need to show that the entry $d E_{3}^{+} d \boldsymbol{L} \boldsymbol{\Gamma}$ is non-vanishing to prove Lemma 10.7. It turns out this number is 0.376322 for the first collision and -1.86463 for the second collision.

\section{Appendix A. Delaunay coordinates}

\section{A.1. Elliptic motion}

The material of this section could be found in $[\mathrm{F}]$ and $[\mathrm{W}]$. Consider the 2-body problem with Hamiltonian

$$
H(P, Q)=\frac{|P|^{2}}{2 m}-\frac{k}{|Q|}, \quad(P, Q) \in \mathbb{R}^{4} .
$$

This system is integrable in the Liouville-Arnold sense when $H<0$. So, we can introduce the action-angle variables $(L, \ell, G, g)$ in which the Hamiltonian can be written as

$$
H(L, \ell, G, g)=-\frac{m k^{2}}{2 L^{2}}, \quad(L, \ell, G, g) \in T^{*} \mathbb{T}^{2} .
$$


The Hamiltonian equations are

$$
\dot{L}=\dot{G}=\dot{g}=0, \quad \dot{\ell}=\frac{m k^{2}}{L^{3}} .
$$

We introduce the following notations: $E$ is the energy, $M$ the angular momentum, $e$ the eccentricity, $a$ the semimajor axis, $b$ the semiminor axis.

Then, we have the following relations which explain the physical and geometrical meaning of the Delaunay coordinates:

$$
a=\frac{L^{2}}{m k}, \quad b=\frac{L G}{m k}, \quad E=-\frac{k}{2 a}, \quad-M=G, \quad e=\sqrt{1-\left(\frac{G}{L}\right)^{2}} .
$$

Moreover, $g$ is the argument of apapsis, $\ell$ is called the mean anomaly, and $\ell$ can be related to the polar angle $\psi$ through the equations

$$
\tan \left(\frac{\psi}{2}\right)=\sqrt{\frac{1+e}{1-e}} \cdot \tan \left(\frac{u}{2}\right) \text { and } u-e \sin u=\ell .
$$

We also have Kepler's law

$$
\frac{a^{3}}{T^{2}}=\frac{1}{(2 \pi)^{2}}
$$

which relates the semimajor axis $a$ and the period $T$ of the ellipse.

Denoting the particle's position by $Q=\left(q_{1}, q_{2}\right)$ and its momentum by $P=\left(p_{1}, p_{2}\right)$, we have the following formulas in the case $g=0$ :

$$
\begin{gathered}
q_{1}=\frac{L^{2}}{m k}\left(\cos u-\sqrt{1-\frac{G^{2}}{L^{2}}}\right), \quad q_{2}=\frac{L G}{m k} \sin u, \\
p_{1}=-\frac{m k}{L} \frac{\sin u}{1-\sqrt{1-G^{2} / L^{2}} \cos u}, \quad p_{2}=\frac{m k}{L^{2}} \frac{G \cos u}{1-\sqrt{1-G^{2} / L^{2}} \cos u},
\end{gathered}
$$

where $u$ and $\ell$ are related by $u-e \sin u=\ell$. Here, $g$ does not appear because the argument of apapsis is chosen to be zero. In the general case, we need to rotate the $\left(q_{1}, q_{2}\right)$ and $\left(p_{1}, p_{2}\right)$ using the matrix

$$
\left[\begin{array}{cc}
\cos g & -\sin g \\
\sin g & \cos g
\end{array}\right]
$$

Notice that the equation (A.1) describes an ellipse with one focus at the origin and the other focus on the negative $x$-axis. We want to be consistent with [G2], i.e. we want $g=\frac{1}{2} \pi$ to correspond to the "vertical" ellipse with one focus at the origin and the other focus on the positive $y$-axis. Therefore, we rotate the picture clockwise. So, we use the Delaunay coordinates which are related to the Cartesian ones through the equations

$$
\begin{aligned}
Q_{\|} & =\frac{1}{m k}\left(L^{2}\left(\cos u-\sqrt{1-\frac{G^{2}}{L^{2}}}\right) \cos g+L G(\sin u) \sin g\right), \\
Q_{\perp} & =\frac{1}{m k}\left(-L^{2}\left(\cos u-\sqrt{1-\frac{G^{2}}{L^{2}}}\right) \sin g+L G(\sin u) \cos g\right) .
\end{aligned}
$$

This is an ellipse focused at the origin with its other focus lying on the positive $y$-axis. 


\section{A.2. Hyperbolic motion}

The above formulas can also be used to describe hyperbolic motion, where we need to replace "sin" by "sinh" and "cos" by "cosh", and change signs properly (cf. [F], [W]). Namely, we have $a=L^{2} / m k, b=L G / m k, E=k / 2 a,-M=G$ and $e=\sqrt{1+(G / L)^{2}}$. Also,

$$
\begin{array}{ll}
q_{1}=\frac{L^{2}}{m k}\left(\cosh u-\sqrt{1+\frac{G^{2}}{L^{2}}}\right), & q_{2}=\frac{L G}{m k} \sinh u, \\
p_{1}=-\frac{m k}{L} \frac{\sinh u}{1-\sqrt{1+G^{2} / L^{2}} \cosh u}, & p_{2}=-\frac{m k}{L^{2}} \frac{G \cosh u}{1-\sqrt{1+G^{2} / L^{2}} \cosh u} .
\end{array}
$$

where $u$ and $\ell$ are related by

$$
u-e \sinh u=\ell, \quad \text { where } e=\sqrt{1+\left(\frac{G}{L}\right)^{2}} .
$$

This hyperbola is symmetric with respect to the $x$-axis, opens to the right, and the particle moves counterclockwise on it when $u$ increases ( $\ell$ decreases) in the case when minus the angular momentum $G=P \times Q<0$. The angle $g$ is defined to be the angle measured from the positive $x$-axis to the symmetric axis. There are two such angles that differ by $\pi$ depending on the orientation of the symmetric axis. This $\pi$ difference disappears after taking tan, or in the symplectic form and the Hamiltonian equation after taking derivative, so it does not matter which angle we choose.

When the particle moves to the right of the sections $\left\{x_{4, \|}^{R}=-\frac{1}{2} \chi\right\}$ and $\left\{x_{4, \|}^{L}=\frac{1}{2} \chi\right\}$ (Definition 4.3 and Figure 3), we have a hyperbola opening to the left and the particle moves counterclockwise. To achieve this, we rotate (A.3) by an angle $\pi+g$. In this case, we choose $g$ to be the angle measured from the positive $x$-axis to the symmetric axis pointing to the perigee:

$$
\begin{aligned}
& Q_{\|}=\frac{1}{m k}\left(-(\cos g) L^{2}(\cosh u-e)+(\sin g) L G \sinh u\right), \\
& Q_{\perp}=\frac{1}{m k}\left(-(\sin g) L^{2}(\cosh u-e)-(\cos g) L G \sinh u\right) . \\
& P=\frac{m k}{1-e \cosh u}\left(\frac{1}{L}(\sinh u) \cos g-\frac{G}{L^{2}}(\sin g) \cosh u,\right. \\
&\left.\frac{1}{L}(\sinh u) \sin g+\frac{G}{L^{2}}(\cos g) \cosh u\right) .
\end{aligned}
$$

We see from (A.4), when $|u|$ is large, that we have $\operatorname{sign}(u)=-\operatorname{sign}(\ell)$. We have three different choices of $g$ in this paper:

(a) When the particle $Q_{4}$ is moving to the right of the sections $\left\{x_{4, \|}^{R}=-\frac{1}{2} \chi\right\}$ and $\left\{x_{4, \|}^{L}=\frac{1}{2} \chi\right\}$, and if the incoming asymptote is horizontal, (see the arrows in Figures 1 
and 2 for "incoming" and "outgoing"), then the particle comes from the left, and as $u$ tends to $-\infty$, the $y$-coordinate is bounded and the $x$-coordinate is negative. In this case, we have $\tan g=G / L, g \in\left(-\frac{1}{2} \pi, 0\right)$. We use $u<0$ to refer to this piece of orbit.

(b) When the particle $Q_{4}$ is moving to the right of the sections $\left\{x_{4, \|}^{R}=-\frac{1}{2} \chi\right\}$ and $\left\{x_{4, \|}^{L}=\frac{1}{2} \chi\right\}$, and if the outgoing asymptote is horizontal, then the particle escapes to the left, and as $u$ tends to $\infty$, the $y$-coordinate is bounded and the $x$-coordinate is negative. In this case, we have $\tan g=-G / L, g \in\left(0, \frac{1}{2} \pi\right)$. We use $u>0$ to refer to this piece of orbit. The above two cases can be unified as

$$
\tan g=-\operatorname{sign}(u) \frac{G}{L}, \text { with } G<0, L>0 .
$$

(c) When the particle $Q_{4}$ is moving to the left of the sections $\left\{x_{4, \|}^{R}=-\frac{\chi}{2}\right\}$ and $\left\{x_{4, \|}^{L}=\frac{\chi}{2}\right\}$, we treat the motion as hyperbolic motion focused at $Q_{1}$. We move the origin to $Q_{1}$. The hyperbola opens to the right. The particle $Q_{4}$ moves on the hyperbola counterclockwise with negative angular momentum $G$, we then rotate by angle $g$, and $g$ is the angle measured from the positive $x$-axis to the symmetric axis pointing to the opening of the hyperbola. The orbit has the following parametrization:

$$
\begin{gathered}
Q=\frac{1}{m k}\left((\cos g) L^{2}(\cosh u-e)-(\sin g) L G \sinh u,(\sin g) L^{2}(\cosh u-e)+(\cos g) L G \sinh u\right), \\
P=\frac{m k}{1-e \cosh u}\left(-\frac{1}{L}(\sinh u) \cos g+\frac{G}{L^{2}}(\sin g) \cosh u,\right. \\
\left.-\frac{1}{L}(\sinh u) \sin g-\frac{G}{L^{2}}(\cos g) \cosh u\right) .
\end{gathered}
$$

We note that the Delaunay coordinates have some singular behavior near double collision. When we set $e=1$ in (A.4), we find $\ell=u^{3}+$ h.o.t. Hence, $u$ as a function $\ell$ in a neighborhood of zero is only $C^{0}$ but not $C^{1}$. One can verify that, for $G=0$ and $\ell \neq 0$, the hyperbolic Delaunay coordinates still give a symplectic transformation, so we only have singular behavior when $G$ and $\ell$ are both close to zero. To control this singular behavior, we need the following estimates in Lemma 7.3.

Lemma A.1. (Lemma A.1 of [DX]) In the hyperbolic Delaunay coordinates, as $G \rightarrow 0$, $u \rightarrow 0$ and $L$ being close to 1 , we have the following estimates of the first-order derivatives

$$
\left|\frac{\partial u}{\partial G}\right| \leqslant 2 \quad \text { and } \quad\left|\frac{\partial u}{\partial L}\right| \leqslant 2|G|
$$

and the second-order derivatives

$$
\left|\frac{\partial Q}{\partial u} \frac{\partial^{2} u}{\partial G^{2}}\right| \leqslant 4, \quad\left|\frac{\partial Q}{\partial u} \frac{\partial^{2} u}{\partial L^{2}}\right| \leqslant 4 G^{2} \quad \text { and } \quad\left|\frac{\partial Q}{\partial u} \frac{\partial^{2} u}{\partial G \partial L}\right| \leqslant 4|G| .
$$


We next cite Lemma A.2 of [DX] to simplify our calculation. The lemma implies that we can replace $\pm u$ by $\ln (\mp \ell / e)$ when taking first- and second-order derivatives.

Lemma A.2. (Lemma A.2 of [DX]) Let $u$ be the function of $\ell, G$ and $L$ given by (A.4), and let $\sigma=\operatorname{sign}(u)$ when $|u|$ is large. Then, we can approximate $u$ by $\ln (-\sigma \ell / e)$ in the following sense:

$$
\begin{gathered}
\sigma u-\ln \frac{-\sigma \ell}{e}=O\left(\frac{\ln |\ell|}{\ell}\right), \quad \frac{\partial u}{\partial \ell}=\frac{\sigma}{\ell}+O\left(\frac{1}{\ell^{2}}\right) \\
\left(\frac{\partial}{\partial L}, \frac{\partial}{\partial G}\right)(u+\sigma \ln e)=O\left(\frac{1}{|\ell|}\right), \quad\left(\frac{\partial}{\partial L}, \frac{\partial}{\partial G}\right)^{2}(u+\sigma \ln e)=O\left(\frac{1}{|\ell|}\right),
\end{gathered}
$$

The estimates above are uniform, as long as $|G| \leqslant C, 1 / C \leqslant L \leqslant C$ and $\ell>\ell_{0}$ for some constant $C>1$ and the implied constants in $O(\cdot)$ depend only on $C$ and $\ell_{0}$.

\section{A.3. The derivative of Cartesian with respect to Delaunay}

Next, we calculate the first-order derivatives of the Cartesian variables with respect to the Delaunay variables. The assumption of the next lemma is met by Lemma 6.11.

Lemma A.3. Assume that $|G| \leqslant C$ and $1 / C \leqslant L \leqslant C$ for some $C>1$.

(a) Assume further in the right case $g=-\sigma \arctan (G / L)+\varepsilon$, where $\sigma=\operatorname{sign}(u)$ and

$$
\varepsilon=O\left(\frac{\mu}{\left|\ell_{4}\right|^{2}+1}+\frac{1}{\chi}\right)
$$

Then, we have the following estimate of the derivative of Cartesian coordinates with respect to the Delaunay coordinates, as $\ell \rightarrow \infty$ :

$$
\frac{\partial\left(Q_{\|}, Q_{\perp}, P_{\|}, P_{\perp}\right)}{\partial(L, \ell, G, g)}=\mathcal{D}+\varepsilon \cdot\left[\begin{array}{cc}
\operatorname{Rot}\left(\frac{1}{2} \pi\right) & 0 \\
0 & \operatorname{Rot}\left(\frac{1}{2} \pi\right)
\end{array}\right] \cdot \mathcal{D}+\left[\begin{array}{c}
O(1)_{2 \times 4} \\
0_{2 \times 4}
\end{array}\right]+O\left(\varepsilon^{2}\right) \cdot \mathcal{D},
$$

where

$$
\mathcal{D}=\left[\begin{array}{cccc}
\sigma \frac{2 L \ell}{m k} & \sigma \frac{L^{2}}{m k} & 0 & 0 \\
-\frac{G L^{2} \ell}{m k\left(G^{2}+L^{2}\right)} & 0 & \frac{L^{3} \ell}{m k\left(G^{2}+L^{2}\right)} & \sigma \frac{L^{2} \ell}{m k} \\
\sigma \frac{k m}{L^{2}} & -\frac{k m}{L \ell^{2}} & 0 & 0 \\
\frac{G k m}{L\left(G^{2}+L^{2}\right)} & 0 & -\frac{k m}{\left(G^{2}+L^{2}\right)} & -\sigma \frac{k m}{L}
\end{array}\right]
$$

(b) In the left case, if we assume $g, G=O(1 / \chi)$ and $L=O(1)$, then the estimates of the derivative are obtained by setting $G=O(1 / \chi)$ in the above matrix. 
(c) We have

$\left|\frac{\partial Q}{\partial \ell}\right|=O(1), \quad\left|\frac{\partial Q}{\partial(L, G, g)}\right|=O(\ell), \quad \frac{\partial Q}{\partial g} \cdot Q=0 \quad$ and $\quad \frac{\partial Q}{\partial G} \cdot Q=O_{C^{2}(L, G, g)}(\ell)$.

Proof. First we drop $e$ in (A.5), since it will contribute a $O(1)$ term in (A.7).

To obtain the leading term we just need to calculate

$$
\frac{\partial\left(\widetilde{Q}_{\|}, \widetilde{Q}_{\perp}, P_{\|}, P_{\perp}\right)}{\partial(L, \ell, G, g)}\left(L, \ell, G,-\sigma \arctan \left(\frac{G}{L}\right)\right),
$$

where $\widetilde{Q}$ refers to the right-hand side of (A.5) with the $e$ term discarded. This derivative is obtained by a straightforward calculation using the formulas (A.5) and (A.6) with the help of Lemma A.2. The calculations of $\partial \widetilde{Q} / \partial G$ and $\partial \widetilde{Q} / \partial L$ are presented in detail in Lemma A.3 of [DX], and the other derivatives are similar. To get the first correction term, i.e. the

$$
O\left(\frac{\mu}{\left|\ell_{4}\right|^{2}+1}+\frac{1}{\chi}\right)
$$

part, let $g_{0}= \pm \arctan (G / L)$ and $\varepsilon=g-g_{0}$. We use the relations

$$
\left(\widetilde{Q}_{\|}, \widetilde{Q}_{\perp}, P_{\|}, P_{\perp}\right)(L, \ell, G, g)=\operatorname{Rot}(g)\left(\widetilde{Q}_{\|}, \widetilde{Q}_{\perp}, P_{\|}, P_{\perp}\right)(L, \ell, G, 0),
$$

and

$$
\operatorname{Rot}\left(g_{0}+\varepsilon\right)=\operatorname{Rot}\left(g_{0}\right)+\varepsilon \operatorname{Rot}\left(\frac{1}{2} \pi\right) \operatorname{Rot}\left(g_{0}\right)+O\left(\varepsilon^{2}\right),
$$

and notice that rotation by $\frac{1}{2} \pi$ has the effect of interchanging the roles of $\|$ and $\perp$. This gives parts (a) and (b) of the lemma.

Part (c) follows by direct calculation from (A.5) and Lemma A.2 (see Lemma A.3 (a) of $[\mathrm{DX}])$.

Remark A.4. (1) Part (c) of Lemma A.3 means $\partial Q / \partial g$ is almost parallel to $\partial Q / \partial G$. This plays an important role in our proof of Lemma 6.5, as well as in [DX]. In fact, in (A.7) the matrix has determinant 1 , since it is symplectic. We look at the $\mathcal{D}$ term in (A.7). The discussion remains true when the other terms are included. In $\mathcal{D}$, the first, second and fourth columns have no obvious linear relations. However, the first and fourth columns have modulus $O(\ell)$, when $|\ell|$ is large. So, the third column must be almost parallel to either the first or fourth column to get determinant 1 .

(2) The second and fourth rows of $\mathcal{D}$ are almost parallel for similar reasons. This fact plays an important role in the proof of Sublemma 9.1.

(3) The same argument can be applied to the inverse of the left-hand side of (A.7). We will see in Lemma A.5 below that the two rows $\partial g / \partial(Q, P)$ and $\partial G / \partial(Q, P)$ are almost parallel, which is used in the proof of Sublemma 9.2 to get the tensor structure. 


\section{A.4. The derivative of Delaunay with respect to Cartesian}

We could have inverted the matrix (A.7) to get the result of this section. However, though the matrix (A.7) is non-singular, it is close to be singular, since we have some large entries of $O(\chi)$. Therefore, we calculate the derivatives $\partial(L, G, g) / \partial(Q, P)$ directly using known identities.

Lemma A.5. We have the following estimates about the derivatives of Delaunay variables with respect to the Cartesian variables:

$$
\frac{\partial L}{\partial(Q, P)}=-\frac{L^{3}}{m k^{2}}\left(\frac{k Q}{|Q|^{3}}, \frac{P}{m}\right), \quad \frac{\partial G}{\partial(Q, P)}=\left(-P_{\perp}, P_{\|}, Q_{\perp},-Q_{\|}\right),
$$

and

$\frac{\partial g}{\partial(Q, P)}=\frac{1}{|P|^{2}}\left(0,0,-P_{\perp}, P_{\|}\right)-\operatorname{sign}(u)\left(\frac{L}{G^{2}+L^{2}} \frac{\partial G}{\partial(Q, P)}-\frac{G}{G^{2}+L^{2}} \frac{\partial L}{\partial(Q, P)}\right)+O\left(\frac{1}{\ell^{2}}\right)$,

as $|\ell| \rightarrow \infty$.

Proof. From the relation

$$
\frac{m k^{2}}{2 L^{2}}=\frac{P^{2}}{2 m}-\frac{k}{|Q|}
$$

we get

$$
\frac{\partial L}{\partial(Q, P)}=-\frac{L^{3}}{m k^{2}}\left(\frac{k Q}{|Q|^{3}}, \frac{P}{m}\right)
$$

We also have

$$
G=P \times Q \quad \text { and } \quad \frac{\partial G}{\partial(Q, P)}=\left(-P_{\perp}, P_{\|}, Q_{\perp},-Q_{\|}\right) .
$$

To get the derivative $\partial g / \partial(Q, P)$, we take the quotient $P_{\perp} / P_{\|}$in (A.5) and (A.6), then apply the formula

$$
\tan (\alpha \pm \beta)=\frac{\tan \alpha \pm \tan \beta}{1 \mp \tan \alpha \tan \beta}
$$

to get that, as $|u| \rightarrow \infty$,

$$
g=\arctan \left(\frac{P_{\perp}}{P_{\|}}\right)-\operatorname{sign}(u) \arctan \left(\frac{G}{L}\right)-e^{-2|u|} E\left(\frac{G}{L}, g, u\right)+o\left(e^{-2|u|}\right) \quad(\bmod \pi),
$$

where $E$ is a smooth function. Hence,

$$
\begin{aligned}
\partial g & =\frac{P_{\|} \partial P_{\perp}-P_{\perp} \partial P_{\|}}{P_{\|}^{2}+P_{\perp}^{2}}-\operatorname{sign}(u) \frac{L \partial G-G \partial L}{G^{2}+L^{2}}+O\left(\frac{1}{\ell^{2}}\right) \\
& =\frac{1}{P^{2}}\left(0,0,-P_{\perp}, P_{\|}\right)-\operatorname{sign}(u) \frac{L \partial G-G \partial L}{G^{2}+L^{2}}+O\left(\frac{1}{\ell^{2}}\right) .
\end{aligned}
$$




\section{A.5. Second-order derivatives}

The following estimates of the second-order derivatives are used in integrating the variational equation.

Lemma A.6. (Lemma A.5 of [DX]) Assume that $|G| \leqslant C$ and $1 / C \leqslant L \leqslant C$ for some constant $C>1$.

(a) We have, as $|\ell| \rightarrow \infty$,

$$
\begin{gathered}
\frac{\partial^{2} Q}{\partial g^{2}}=-Q, \quad \frac{\partial^{2} Q}{\partial g \partial G}+\frac{\partial Q}{\partial G}, \quad\left(\frac{\partial}{\partial G}, \frac{\partial}{\partial g}\right)\left(\frac{\partial|Q|^{2}}{\partial g}\right)=(0,0), \\
\frac{\partial^{2} Q}{\partial G^{2}}=O(\ell), \quad \frac{\partial^{2} Q}{\partial L^{2}}=O(\ell), \quad \frac{\partial^{2} Q}{\partial L \partial G}=O(\ell) .
\end{gathered}
$$

(b) Under the conditions of Lemma A.3 (a), we have

$$
\begin{aligned}
\frac{\partial^{2} Q}{\partial G^{2}} & =\frac{L^{2}}{\left(L^{2}+G^{2}\right)^{3 / 2}}(L \cosh u, 2 G \sinh u)+O(1), \\
\frac{\partial^{2} Q}{\partial g \partial G} & =\left(\frac{L^{2} \sinh u}{\sqrt{L^{2}+G^{2}}}, 0\right)+O(1), \\
\frac{\partial^{2} Q}{\partial g \partial L} & =\left(-\frac{G L \sinh u}{\sqrt{L^{2}+G^{2}}},-2 \sqrt{L^{2}+G^{2}} \cosh u\right)+O(1), \\
\frac{\partial^{2} Q}{\partial G \partial L} & =\frac{-L}{\left(L^{2}+G^{2}\right)^{3 / 2}}\left(L G \cosh u,\left(L^{2}+3 G^{2}\right) \sinh u\right)+O(1) .
\end{aligned}
$$

(c) Under the conditions of Lemma A.3 (b), we have

$$
\begin{aligned}
\frac{\partial^{2} Q}{\partial G^{2}} & =-\cosh u(1,0)+O(1), \quad \frac{\partial^{2} Q}{\partial g \partial G}=-L \sinh u(1,0)+O(1), \\
\frac{\partial^{2} Q}{\partial g \partial L} & =L \sinh u(0,2)+O(1), \quad \frac{\partial^{2} Q}{\partial G \partial L}=\cosh u(0,1)+O(1) .
\end{aligned}
$$

\section{Appendix B. Gerver's mechanism}

\section{B.1. Gerver's result in [G2]}

We summarize the result of [G2] in Table 1. Recall that the Gerver scenario deals with the limiting case $\chi \rightarrow \infty$ and $\mu \rightarrow 0$. Accordingly, $Q_{1}$ disappears at infinity and there is no interaction between $Q_{3}$ and $Q_{4}$. Hence, both particles perform Kepler motions. The shape of each Kepler orbit is characterized by energy, angular momentum and the argument of apapsis. In Gerver's scenario, the incoming and outgoing asymptotes of the hyperbola are always horizontal and the semimajor axis of the ellipse is always vertical. So, we only need to describe on the energy and angular momentum. 


\begin{tabular}{|c|c|c|c|c|}
\hline & 1st collision & $@\left(-\varepsilon_{0} \varepsilon_{1}, \varepsilon_{0}+\varepsilon_{1}\right)$ & 2nd collision & $@\left(\varepsilon_{0}^{2}, 0\right)$ \\
\hline & $Q_{3}$ & $Q_{4}$ & $Q_{3}$ & $Q_{4}$ \\
\hline energy & $-\frac{1}{2}$ & $\frac{1}{2}$ & $-\frac{1}{2} \mapsto-\frac{\varepsilon_{1}^{2}}{2 \varepsilon_{0}^{2}}$ & $\frac{1}{2} \mapsto \frac{\varepsilon_{1}^{2}}{2 \varepsilon_{0}^{2}}$ \\
\hline angular momentum & $\varepsilon_{1} \mapsto-\varepsilon_{0}$ & $p_{1} \mapsto-p_{2}$ & $-\varepsilon_{0}$ & $\varepsilon_{0} \sqrt{2}$ \\
\hline eccentricity & $\varepsilon_{0} \mapsto \varepsilon_{1}$ & & $\varepsilon_{1} \mapsto \varepsilon_{0}$ & \\
\hline semimajor & 1 & -1 & $1 \mapsto\left(\frac{\varepsilon_{0}}{\varepsilon_{1}}\right)^{2}$ & $1 \mapsto-\frac{\varepsilon_{1}^{2}}{\varepsilon_{0}^{2}}$ \\
\hline semiminor & $\varepsilon_{1} \mapsto \varepsilon_{0}$ & $p_{1} \mapsto p_{2}$ & $\varepsilon_{0} \mapsto \frac{\varepsilon_{0}^{2}}{\varepsilon_{1}}$ & $\varepsilon_{0} \sqrt{2} \mapsto \varepsilon_{1} \sqrt{2}$ \\
\hline
\end{tabular}

Table 1. Summary of the result of [G2].

Here,

$$
p_{1,2}=\frac{-Y \pm \sqrt{Y^{2}+4(X+R)}}{2}
$$

$R=\sqrt{X^{2}+Y^{2}}$ and $(X, Y)$ stands for the point where collision occurs (the parenthesis after @ in the table). We will call the two points the Gerver collision points. In the above table $\varepsilon_{0}$ is a free parameter and $\varepsilon_{1}=\sqrt{1-\varepsilon_{0}^{2}}$. At the collision points, the velocities of the particles are the following. For the first collision,

$$
\begin{array}{ll}
v_{3}^{-}=\left(-\frac{\varepsilon_{1}^{2}}{\varepsilon_{0} \varepsilon_{1}+1},-\frac{\varepsilon_{0}}{\varepsilon_{0} \varepsilon_{1}+1}\right), & v_{4}^{-}=\left(1-\frac{Y}{R p_{1}}, \frac{1}{R p_{1}}\right), \\
v_{3}^{+}=\left(\frac{\varepsilon_{0}^{2}}{\varepsilon_{0} \varepsilon_{1}+1}, \frac{\varepsilon_{1}}{\varepsilon_{0} \varepsilon_{1}+1}\right), & v_{4}^{+}=\left(-1+\frac{Y}{R p_{2}},-\frac{1}{R p_{2}}\right) .
\end{array}
$$

For the second collision,

$$
v_{3}^{-}=\left(-\frac{\varepsilon_{1}}{\varepsilon_{0}},-\frac{1}{\varepsilon_{0}}\right), \quad v_{4}^{-}=\left(1, \frac{\sqrt{2}}{\varepsilon_{0}}\right), \quad v_{3}^{+}=\left(1,-\frac{1}{\varepsilon_{0}}\right) \quad \text { and } \quad v_{4}^{+}=\left(-\frac{\varepsilon_{1}}{\varepsilon_{0}}, \frac{\sqrt{2}}{\varepsilon_{0}}\right) \text {. }
$$




\section{Appendix C. $\mathscr{C}^{1}$ control of the global map: proof of Lemma 3.2}

In this appendix, we derive Lemma 3.2 from Proposition 5.2. We split the proof into six steps.

Step 0. (Preparations: definitions of auxiliary vectors and simplification of the five matrices.)

We define some new auxiliary vectors. Recall that in the paragraph before Proposition 5.2, we introduced a convention to use bold font to indicate that the estimate of the corresponding entry is actually $\sim$, not only $\lesssim$. Below, we use the following notational convention to make it easier for the reader to keep track of the computations.

Notation C.1. A vector with "tilde", "hat" and "bar" means a $O(1 / \chi), O(\mu)$ and $O(1)$ perturbation to the vector, respectively.

Definition C.2. We define the following list of vectors:

- $\tilde{u}:=N_{3} u=u+O\left(\frac{1}{\chi}\right) \lesssim\left(\frac{1}{\chi^{2}},-1, \frac{1}{\chi^{2}}, \frac{1}{\chi^{2}} ; \mu, \frac{\mu}{\chi}, \frac{1}{\mu \chi^{2}}, \frac{\mu}{\chi^{2}} ; \frac{\mu}{\chi}, \frac{\mu}{\chi}\right)^{T}$ $\tilde{l}:=l N_{3}=l+O\left(\frac{1}{\chi}\right) \lesssim\left(1, \frac{1}{\chi^{2}}, \frac{1}{\chi^{2}}, \frac{1}{\chi^{2}} ; \frac{1}{\mu \chi^{2}}, \frac{1}{\chi^{2}}, \mu, \frac{\mu}{\chi} ; \frac{1}{\chi}, \frac{1}{\chi}\right) ;$

- $\hat{u}:=N_{5} u=u+O(\mu) \lesssim\left(\mu,-\mathbf{1}, \mu, \mu ; \mu, \frac{\mu}{\chi}, \frac{\mu}{\chi}, \frac{\mu}{\chi} ; \mu, \mu\right)$,

$$
\hat{l}:=l N_{1}=l+O(\mu) \lesssim\left(\mathbf{1}, \mu, \mu, \mu ; \frac{\mu}{\chi}, \frac{\mu}{\chi}, \mu, \frac{\mu}{\chi} ; \mu, \mu\right) ;
$$

- $\delta u:=A L \cdot R^{-1} u_{i} \sim A R \cdot L^{-1} u_{i^{\prime}} \lesssim\left(0,0,0,0 ; 0, \mu, 0, \frac{1}{\chi} ; 0, \frac{1}{\chi}\right)^{T}=O(\mu)$;

$$
\delta l:=l_{i i i} L \cdot R^{-1} C \sim l_{i i i^{\prime}} R \cdot L^{-1} C \lesssim\left(\frac{1}{\chi}, \frac{1}{\chi^{4}}, \frac{1}{\chi^{4}}, \frac{1}{\chi^{4}} ; \frac{1}{\chi^{2}}, \frac{1}{\chi}, \frac{\mu}{\chi}, \mu ; \frac{1}{\chi}, \frac{1}{\chi}\right)=O(\mu)
$$

- $\hat{u}_{i i i}:=u_{i i i}+\delta u \lesssim\left(0,0,0,0 ; 0, \mu, 0, \frac{1}{\chi} ; 1,1\right)^{T}$

$$
\hat{l}_{i}:=l_{i}+\delta l \lesssim\left(1, \frac{1}{\chi^{3}}, \frac{1}{\chi^{3}}, \frac{1}{\chi^{3}} ; \frac{1}{\mu \chi^{2}}, \frac{1}{\chi}, \mu, \mu ; 1,1\right)
$$

- $\hat{u}_{i i i^{\prime}}:=u_{i i i^{\prime}}+\delta u \lesssim\left(0,0,0,0 ; 0, \mu, 0, \frac{1}{\chi} ; 1,1\right)^{T}$,

$$
\hat{l}_{i^{\prime}}:=l_{i^{\prime}}+\delta l \lesssim\left(\frac{1}{\chi}, \frac{1}{\chi^{4}}, \frac{1}{\chi^{4}}, \frac{1}{\chi^{4}} ; \frac{1}{\chi^{2}}, \frac{1}{\chi}, \frac{\mu}{\chi}, \mu ; 1,1\right) ;
$$

and the following matrices:

$$
S_{2}:=A L \cdot R^{-1} C \quad \text { and } \quad S_{4}:=A R \cdot L^{-1} C .
$$


Note that $S_{2}, S_{4} \lesssim S$, where

$$
S:=\left[\begin{array}{cc|cccc|c}
\multicolumn{2}{c|}{\operatorname{Id}_{4 \times 4}} & 0_{4 \times 1} & 0_{4 \times 1} & 0_{4 \times 1} & 0_{4 \times 1} & 0_{4 \times 2} \\
\hline 0 & 0_{1 \times 3} & 1+O(\mu) & 0 & 0 & 0 & 0_{1 \times 2} \\
0 & 0_{1 \times 3} & 0 & 1+O(\mu) & 0 & 0 & O(\mu)_{1 \times 2} \\
O(1) & O\left(1 / \chi^{3}\right)_{1 \times 3} & O\left(1 / \mu \chi^{2}\right) & O\left(1 / \mu \chi^{3}\right) & 1+O(\mu) & O(\mu / \chi) & O(1 / \chi)_{1 \times 2} \\
0 & 0_{1 \times 3} & 0 & 0 & 0 & 1+O(\mu) & 0_{1 \times 2} \\
\hline 0 & 0_{1 \times 3} & 0 & 0 & 0 & 0 & 0_{1 \times 2} \\
1+O(\mu) & O\left(1 / \chi^{3}\right)_{1 \times 3} & O\left(1 / \mu \chi^{2}\right) & O\left(1 / \chi^{2}\right) & O(\mu) & O(\mu) & O(1 / \chi)_{1 \times 2}
\end{array}\right] .
$$

Sublemma C.3. (1)

$$
l \cdot u, \tilde{l} \cdot u, l \cdot \tilde{u} \lesssim \frac{1}{\chi^{2}}
$$

$$
l_{i i i} \cdot L \cdot R^{-1} u_{i}=\frac{1+O(\mu)}{\chi} \quad \text { and } \quad l_{i i i^{\prime}} \cdot R \cdot L^{-1} u_{i^{\prime}}=-\frac{1+O(\mu)}{\chi} .
$$

Proof. All of these estimates come from straightforward calculations using Proposition 5.2 and Definition C.2. Item (2) is exact. It uses Proposition 5.2 (b2).

Using Definition C.2 and Sublemma C.3, we simplify the five matrices into sums as follows. Notice that the factors $\left(\operatorname{Id}_{10}+u_{1}^{\mathrm{i}} \otimes l_{1}^{\mathrm{i}}\right)$ in $(\mathrm{I})$ and $\operatorname{Id}_{10}+u_{5}^{\mathrm{f}} \otimes l_{5}^{\mathrm{f}}$ in $(\mathrm{V})$ are both $O(1)$, so we do not include them in the following calculation until the final step for simplicity. We shall write $(\overline{\mathrm{I}})$ and $(\overline{\mathrm{V}})$ for the modified matrices. We have

$$
\begin{aligned}
(\mathrm{III}) & \lesssim\left(\operatorname{Id}_{10}+\chi u \otimes l\right) N_{3}\left(\operatorname{Id}_{10}+\chi u \otimes l^{\prime}\right) \\
& =\left(\operatorname{Id}_{10}+\chi u \otimes l\right)\left(N_{3}+\chi N_{3} u \otimes l^{\prime}\right) \\
& =\left(\operatorname{Id}_{10}+\chi u \otimes l\right)\left(N_{3}+\chi \tilde{u} \otimes l^{\prime}\right) \\
& =\left(N_{3}+\chi \tilde{u} \otimes l^{\prime}\right)+\chi u \otimes l\left(N_{3}+\chi \tilde{u} \otimes l^{\prime}\right) \\
& \sim N_{3}+\chi \tilde{u} \otimes l^{\prime}+\chi u \otimes \tilde{l}, \\
(\mathrm{II}) & =\left(\chi u_{i i i} \otimes l_{i i i}+A\right) L \cdot R^{-1}\left(\chi u_{i} \otimes l_{i}+C\right) \\
& =\chi^{2} u_{i i i} \otimes l_{i i i} L \cdot R^{-1} u_{i} \otimes l_{i}+\chi \delta u \otimes l_{i}+\chi u_{i i i} \otimes \delta l+S \\
& \sim \chi \hat{u}_{i i i} \otimes \hat{l}_{i}-\chi \delta u \otimes \delta l+S, \\
(\mathrm{IV}) & \sim \chi \hat{u}_{i i i^{\prime}} \otimes \hat{l}_{i^{\prime}}-\chi \delta u \otimes \delta l+S, \\
(\overline{\mathrm{I}}) & \lesssim\left(\operatorname{Id}_{10}+\chi u \otimes l\right) N_{1}=N_{1}+\chi u \otimes l N_{1}=N_{1}+\chi u \otimes \hat{l}, \\
(\overline{\mathrm{V}}) & \lesssim N_{5}\left(\operatorname{Id}_{10}+\chi u \otimes l^{\prime}\right)=N_{5}+N_{5} \chi u \otimes l^{\prime}=N_{5}+\chi \hat{u} \otimes l^{\prime},
\end{aligned}
$$


where, for (III), we used that

$$
l\left(N_{3}+\chi \tilde{u} \otimes l^{\prime}\right) \lesssim \tilde{l}+\frac{1}{\chi} l^{\prime} \sim \tilde{l},
$$

by Definition C.2 (first bullet point) and Sublemma C.3 (1).

Step 1. (Decomposing (IV)(III)(II) into three summands.)

We start with an auxillary estimate.

Sublemma C.4. We have the following estimates as $1 / \chi \ll \mu \rightarrow 0$ :

(1)

$$
\begin{aligned}
(\text { III }) \hat{u}_{i i i} & \lesssim\left(\frac{1}{\chi^{2}}, 1, \frac{1}{\chi^{2}}, \frac{1}{\chi^{2}} ; \mu, \mu, \frac{1}{\chi}, \frac{1}{\chi} ; \mathbf{1}, \mathbf{1}\right)^{T}=O(1), \\
\hat{l}_{i^{\prime}}(\mathrm{III}) & \lesssim\left(1, \frac{1}{\chi^{2}}, \frac{1}{\chi^{2}}, \frac{1}{\chi^{2}} ; \frac{1}{\chi}, \frac{1}{\chi}, \mu, \mu ; \mathbf{1}, \mathbf{1}\right)=O(1),
\end{aligned}
$$

(2) $\hat{l}_{i^{\prime}}(\mathrm{III}) \hat{u}_{i i i} \rightarrow-2$.

Corollary C.5. (1) $\delta l(\mathrm{III}) \hat{u}_{i i i} \lesssim 1 / \chi$,

(2) $\hat{l}_{i^{\prime}}(\mathrm{III}) \delta u \lesssim 1 / \chi$.

Proof. All of these are done by straightforward calculation using the information obtained in Proposition 5.2 together with the calculation of (III) in (C.1). The $\mathbf{1}$ entries in item (1) are actually $\left(N_{3}\right)_{44}\left(u_{i i i}(9), u_{i i i}(10)\right)$ and $\left(l_{i^{\prime}}(9), l_{i^{\prime}}(10)\right)\left(N_{3}\right)_{44}$, up to a $O(\mu)$ error. Item (2) is in fact $\left(l_{i^{\prime}}(9), l_{i^{\prime}}(10)\right)\left(N_{3}\right)_{44}\left(u_{i i i}(9), u_{i i i}(10)\right)$, up to a $O(\mu)$ error. These terms can be calculated explicitly using part (b1)-(b3) of Proposition 5.2.

Then, we consider

$$
\begin{aligned}
& (\mathrm{IV})(\mathrm{III})(\mathrm{II}) \\
& \begin{aligned}
\sim & \left(\chi \hat{u}_{i i i^{\prime}} \otimes \hat{l}_{i^{\prime}}-\chi \delta u \otimes \delta l+S\right)(\mathrm{III})\left(\chi \hat{u}_{i i i} \otimes \hat{l}_{i}-\chi \delta u \otimes \delta l+S\right) \\
= & \chi^{2} \hat{u}_{i i i^{\prime}} \otimes \hat{l}_{i^{\prime}}(\mathrm{III}) \hat{u}_{i i i} \otimes \hat{l}_{i}+(-\chi \delta u \otimes \delta l+S)(\mathrm{III})\left(\chi \hat{u}_{i i i} \otimes \hat{l}_{i}\right) \\
& \quad+\left(\chi \hat{u}_{i i i^{\prime}} \otimes \hat{l}_{i^{\prime}}\right)(\mathrm{III})(-\chi \delta u \otimes \delta l+S)+(-\chi \delta u \otimes \delta l+S)(\mathrm{III})(-\chi \delta u \otimes \delta l+S) .
\end{aligned}
\end{aligned}
$$

Define

$$
v=\hat{l}_{i^{\prime}}(\mathrm{III})(-\chi \delta u \otimes \delta l+S) \quad \text { and } \quad v^{\prime}=(-\chi \delta u \otimes \delta l+S)(\mathrm{III}) \hat{u}_{i i i} .
$$

Both are of order 1, by Corollary C.5 and Sublemma C.4 (1). From Sublemma C.4(2), we get

$$
\begin{aligned}
(\mathrm{C} .2) & \sim \chi^{2} \hat{u}_{i i i^{\prime}} \otimes \hat{l}_{i}+\chi v^{\prime} \otimes \hat{l}_{i}+\chi \hat{u}_{i i i^{\prime}} \otimes v+(\chi \delta u \otimes \delta l-S)(\mathrm{III})(\chi \delta u \otimes \delta l-S) \\
& =\chi^{2}\left(\hat{u}_{i i i^{\prime}}+\frac{1}{\chi} v^{\prime}\right) \otimes\left(\hat{l}_{i}+\frac{1}{\chi} v\right)-v^{\prime} \otimes v+(\chi \delta u \otimes \delta l-S)(\mathrm{III})(\chi \delta u \otimes \delta l-S) \\
& =: \chi^{2} \tilde{\hat{u}}_{i i i^{\prime}} \otimes \hat{\hat{l}}_{i}-v^{\prime} \otimes v+(\chi \delta u \otimes \delta l-S)(\mathrm{III})(\chi \delta u \otimes \delta l-S),
\end{aligned}
$$


where we have defined

$$
\tilde{\hat{u}}_{i i i^{\prime}}=u_{i i i^{\prime}}+\delta u+\frac{1}{\chi} v^{\prime} \quad \text { and } \quad \tilde{\hat{l}}_{i}=l_{i}+\delta l+\frac{1}{\chi} v .
$$

It is important to stress that the coefficient of the $\chi^{2}$ term is non-zero.

Next, we consider

$$
(\mathrm{V})(\mathrm{IV})(\mathrm{III})(\mathrm{II})(\mathrm{I})=(\mathrm{V})(\mathrm{C} .3)(\mathrm{I}) \text {. }
$$

In the following, we are going to show that $\chi^{2}(\mathrm{~V}) \tilde{\hat{u}}_{i i i^{\prime}} \otimes \tilde{\hat{l}}_{i}(\mathrm{I})$ gives rise to the $\chi^{2}$ part of the main lemma (Lemma 3.2). The (V) $v^{\prime} \otimes v(\mathrm{I})$ part will be absorbed into $O(\mu \chi)$ part. The last summand in (C.3) will give rise to the $O(\chi)$ part together with a perturbation of order $O(\mu \chi)$, where the $O(\chi)$ part comes from (V)S(III)S(I).

Step 2. (The first summand in (C.3) gives the $O\left(\chi^{2}\right)$ contribution in Lemma 3.2.)

The following sublemma is needed for this step.

Sublemma C.6. (1)

$$
l^{\prime} \cdot \hat{u}_{i i i^{\prime}} \lesssim \frac{1}{\chi} \quad \text { and } \quad \hat{l}_{i} \cdot u \lesssim \frac{1}{\chi^{2}}
$$

$$
l^{\prime} \cdot v^{\prime} \lesssim \frac{\mu}{\chi} \quad \text { and } \quad v \cdot u \lesssim \frac{\mu}{\chi}
$$

We first consider the term $(\overline{\mathrm{V}})\left(\chi^{2} \tilde{\hat{u}}_{i i i^{\prime}} \otimes \tilde{\hat{l}}_{i}\right)(\overline{\mathrm{I}})$. We keep in mind that $N_{1}, N_{5}=O(\mu \chi)$. Define

$$
\begin{aligned}
\bar{u}^{\prime}: & =(\overline{\mathrm{V}}) \tilde{\hat{u}}_{i i i^{\prime}}=N_{5} \tilde{\hat{u}}_{i i i^{\prime}}+\chi \hat{u} \otimes l^{\prime} \cdot \tilde{\hat{u}}_{i i i^{\prime}}=\left(N_{5} \hat{u}_{i i i^{\prime}}+O(\mu)\right)+\hat{u}\left(\chi l^{\prime} \cdot \hat{u}_{i i i^{\prime}}+l^{\prime} \cdot v^{\prime}\right) \\
& =N_{5} \hat{u}_{i i i^{\prime}}+O(1) \hat{u}+O(\mu), \\
\bar{l}^{\prime}: & =\tilde{\hat{l}}_{i}(\overline{\mathrm{I}})=\tilde{\hat{l}}_{i} N_{1}+\chi \tilde{\hat{l}}_{i} \cdot u \otimes \hat{l}=\left(\hat{l}_{i} N_{1}+O(\mu)\right)+\left(\chi \hat{l}_{i} \cdot u+v \cdot u\right) \hat{l}=\hat{l}_{i} N_{1}+O(\mu) .
\end{aligned}
$$

We will analyze $\bar{u}^{\prime}$ and $\bar{l}^{\prime}$ in more detail in the final step.

Step 3. (The second summand $v^{\prime} \otimes v$ in (C.3) gives $(\mathrm{V}) v^{\prime} \otimes v(\mathrm{I})=O(\mu \chi)$.)

The following sublemma is needed in this step.

Sublemma C.7. We have the following estimates:

$$
\begin{aligned}
N_{5} \delta u & \lesssim\left(\frac{\mu}{\chi}, \frac{1}{\chi}, \frac{\mu}{\chi}, \frac{\mu}{\chi} ; \frac{\mu}{\chi}, \mu, \frac{1}{\chi^{2}}, \frac{1}{\chi} ; \frac{1}{\chi}, \frac{1}{\chi}\right)^{T}=O(\mu), \\
\delta l N_{1} & \lesssim\left(\frac{1}{\chi}, \frac{\mu}{\chi}, \frac{\mu}{\chi}, \frac{\mu}{\chi} ; \frac{1}{\chi^{2}}, \frac{1}{\chi}, \frac{\mu}{\chi}, \mu ; \frac{1}{\chi}, \frac{1}{\chi}\right)=O(\mu),
\end{aligned}
$$


(2)

$$
l^{\prime} \cdot \delta u \lesssim \frac{1}{\chi^{2}} \quad \text { and } \quad \delta l \cdot u \lesssim \frac{\mu}{\chi^{2}} .
$$

Before considering $(\overline{\mathrm{V}}) v^{\prime} \otimes v(\overline{\mathrm{I}})$, we perform the following calculation:

$$
\begin{gathered}
(\overline{\mathrm{V}}) \chi \delta u \otimes \delta l=\left(N_{5}+\chi \hat{u} \otimes l^{\prime}\right) \chi \delta u \otimes \delta l=\chi\left(N_{5} \delta u+\chi \hat{u} \otimes l^{\prime} \cdot \delta u\right) \otimes \delta l:=\chi \widehat{\delta u} \otimes \delta l, \\
\chi \delta u \otimes \delta l(\overline{\mathrm{I}})=\chi \delta u \otimes \delta l\left(N_{1}+\chi u \otimes \hat{l}\right)=\chi \delta u \otimes\left(\delta l N_{1}+\chi \delta l \cdot u \otimes \hat{l}\right):=\chi \delta u \otimes \hat{\delta l} .
\end{gathered}
$$

We use Sublemma C.7 to conclude that $\widehat{\delta u}, \widehat{\delta l}=O(\mu)$.

Next, we consider $(\overline{\mathrm{V}}) v^{\prime} \otimes v(\overline{\mathrm{I}})$ :

$$
\begin{aligned}
(\overline{\mathrm{V}}) v^{\prime} \otimes v(\overline{\mathrm{I}}) & \\
= & (\overline{\mathrm{V}})(-\chi \delta u \otimes \delta l+S)(I I I) \hat{u}_{i i i} \otimes \hat{l}_{i^{\prime}}(\mathrm{III})(-\chi \delta u \otimes \delta l+S)(\overline{\mathrm{I}}) \\
= & (-\chi \widehat{\delta u} \otimes \delta l+(\overline{\mathrm{V}}) S)(\mathrm{III}) \hat{u}_{i i i} \otimes \hat{l}_{i^{\prime}}(\mathrm{III})(-\chi \delta u \otimes \widehat{\delta l}+S(\overline{\mathrm{I}})) \\
= & \chi^{2} \widehat{\delta u} \otimes \delta l\left[(\mathrm{III}) \hat{u}_{i i i} \otimes \hat{l}_{i^{\prime}}(\mathrm{III})\right] \delta u \otimes \widehat{\delta l}-\chi \widehat{\delta u} \otimes \delta l\left[(\mathrm{III}) \hat{u}_{i i i} \otimes \hat{l}_{i^{\prime}}(\mathrm{III})\right] S(\overline{\mathrm{I}}) \\
& \quad-\chi(\overline{\mathrm{V}}) S\left[(\mathrm{III}) \hat{u}_{i i i} \otimes \hat{l}_{i^{\prime}}(\mathrm{III})\right] \delta u \otimes \widehat{\delta l}+(\overline{\mathrm{V}}) S\left[(\mathrm{III}) \hat{u}_{i i i} \otimes \hat{l}_{i^{\prime}}(\mathrm{III})\right] S(\overline{\mathrm{I}}) \\
\lesssim & \widehat{\delta u} \otimes \hat{\delta l}+\widehat{\delta u} \otimes \hat{l}_{i^{\prime}}(\mathrm{III}) S(\overline{\mathrm{I}})+(\overline{\mathrm{V}}) S(\mathrm{III}) \hat{u}_{i i i} \otimes \widehat{\delta l}+(\overline{\mathrm{V}}) S(\mathrm{III}) \hat{u}_{i i i} \otimes \hat{l}_{i^{\prime}}(\mathrm{III}) S(\overline{\mathrm{I}})
\end{aligned}
$$

where, in the last step, we use Corollary C.5. The first term above is $O\left(\mu^{2}\right)$. To study the remaining three terms, we continue the calculation in Sublemma C.4 to get the following.

Sublemma C.8. We have the following estimates:

(1)

$$
\begin{aligned}
\hat{l}_{i^{\prime}}(\mathrm{III}) S & \lesssim\left(1, \frac{1}{\chi^{2}}, \frac{1}{\chi^{2}}, \frac{1}{\chi^{2}} ; \frac{1}{\chi}, \frac{1}{\chi}, \mu, \mu ; \frac{1}{\chi}, \frac{1}{\chi}\right)=O(1), \\
\hat{l}_{i^{\prime}}(\mathrm{III}) S N_{1} & \lesssim\left(1, \mu, \mu, \mu ; \frac{1}{\chi}, \frac{1}{\chi}, \mu, \mu ; \mu, \mu\right)=O(1),
\end{aligned}
$$

(2)

$$
\begin{aligned}
S(\mathrm{III}) \hat{u}_{i i i} & \lesssim\left(\frac{1}{\chi^{2}}, 1, \frac{1}{\chi^{2}}, \frac{1}{\chi^{2}} ; \mu, \mu, \frac{1}{\chi}, \frac{1}{\chi} ; 0, \frac{1}{\chi}\right)=O(1), \\
N_{5} S(\mathrm{III}) \hat{u}_{i i i} & \lesssim\left(\mu, 1, \mu, \mu ; \mu, \mu, \frac{1}{\chi}, \frac{1}{\chi} ; \mu, \mu\right)=O(1) .
\end{aligned}
$$

Corollary C.9.

$$
\hat{l}_{i^{\prime}}(\mathrm{III}) S \cdot u \lesssim \frac{\mu}{\chi} \quad \text { and } \quad l^{\prime} \cdot S(\mathrm{III}) \hat{u}_{i i i} \lesssim \frac{\mu}{\chi}
$$


Using Sublemma C.8 and Corollary C.9, we get

$$
\begin{gathered}
\hat{l}_{i^{\prime}}(\mathrm{III}) S(\overline{\mathrm{I}})=\hat{l}_{i^{\prime}}(\mathrm{III}) S N_{1}+\chi \hat{l}_{i^{\prime}}(\mathrm{III}) S \cdot u \otimes \hat{l}=O(1), \\
(\overline{\mathrm{V}}) S(\mathrm{III}) \hat{u}_{i i i}=N_{5} S(\mathrm{III}) \hat{u}_{i i i}+\chi \hat{u} \otimes l^{\prime} \cdot S(\mathrm{III}) \hat{u}_{i i i}=O(1) .
\end{gathered}
$$

Accordingly, the fourth term in (C.6) is $O(1)$, and the other terms are even smaller. Hence,

$$
(\overline{\mathrm{V}}) v^{\prime} \otimes v(\overline{\mathrm{I}})=O(1) \ll O(\mu \chi)
$$

Step 4. (The last summand in (C.3) gives the $O(\chi)$ contribution in Lemma 3.2 and a $O(\mu \chi)$ perturbation.)

To proceed, the following calculation is needed.

Sublemma C.10. We have the following estimates:

(1)

$$
\begin{aligned}
(\mathrm{III}) \delta u & \lesssim\left(\frac{1}{\chi^{3}}, \frac{1}{\chi}, \frac{1}{\chi^{3}}, \frac{1}{\chi^{3}} ; \frac{\mu}{\chi}, \mu, \frac{1}{\chi^{2}}, \frac{1}{\chi} ; \frac{1}{\chi}, \frac{1}{\chi}\right)^{T}=O(\mu), \\
\delta l(\mathrm{III}) & \lesssim\left(\frac{1}{\chi}, \frac{1}{\chi^{3}}, \frac{1}{\chi^{3}}, \frac{1}{\chi^{3}} ; \frac{1}{\chi^{2}}, \frac{1}{\chi}, \frac{\mu}{\chi}, \mu ; \frac{1}{\chi}, \frac{1}{\chi}\right)=O(\mu)
\end{aligned}
$$

(2)

$$
\begin{aligned}
& \delta l(\mathrm{III}) S \lesssim\left(\frac{1}{\chi}, \frac{1}{\chi^{3}}, \frac{1}{\chi^{3}}, \frac{1}{\chi^{3}} ; \frac{1}{\chi^{2}}, \frac{1}{\chi}, \frac{\mu}{\chi}, \mu ; \frac{\mu}{\chi}, \frac{\mu}{\chi}\right)=O(\mu) \\
& \delta l(\mathrm{III}) S N_{1} \lesssim\left(\frac{1}{\chi}, \frac{\mu}{\chi}, \frac{\mu}{\chi}, \frac{\mu}{\chi} ; \frac{1}{\chi^{2}}, \frac{1}{\chi}, \frac{\mu}{\chi}, \mu ; \frac{\mu}{\chi}, \frac{\mu}{\chi}\right)=O(\mu)
\end{aligned}
$$

(3)

$$
\begin{aligned}
& S(\mathrm{III}) \delta u \lesssim\left(\frac{1}{\chi^{3}}, \frac{1}{\chi}, \frac{1}{\chi^{3}}, \frac{1}{\chi^{3}} ; \frac{\mu}{\chi}, \mu, \frac{1}{\chi^{2}}, \frac{1}{\chi} ; 0, \frac{\mu}{\chi}\right)=O(\mu), \\
& N_{5} S(\mathrm{III}) \delta u \lesssim\left(\frac{\mu}{\chi}, \frac{1}{\chi}, \frac{\mu}{\chi}, \frac{\mu}{\chi} ; \frac{\mu}{\chi}, \mu, \frac{1}{\chi^{2}}, \frac{1}{\chi} ; \frac{\mu}{\chi}, \frac{\mu}{\chi}\right)=O(\mu) .
\end{aligned}
$$

Corollary C.11. (1) $\delta l(\mathrm{III}) \delta u \lesssim \mu / \chi$,

(2)

$$
\delta l(\mathrm{III}) S \cdot u \lesssim \frac{\mu}{\chi^{2}} \quad \text { and } \quad l^{\prime} \cdot S(\mathrm{III}) \delta u \lesssim \frac{\mu}{\chi^{2}}
$$


We are now ready to consider the last summand in (C.3). Using (C.5), we get

$$
\begin{aligned}
(\overline{\mathrm{V}}) & (-\chi \delta u \otimes \delta l+S)(\mathrm{III})(-\chi \delta u \otimes \delta l+S)(\overline{\mathrm{I}}) \\
& =(-\chi \widehat{\delta u} \otimes \delta l+(\overline{\mathrm{V}}) S)(\mathrm{III})(-\chi \delta u \otimes \widehat{\delta l}+S(\overline{\mathrm{I}})) \\
& =\chi^{2} \widehat{\delta u} \otimes \delta l(\mathrm{III}) \delta u \otimes \widehat{\delta l}-\chi \widehat{\delta u} \otimes \delta l(\mathrm{III}) S(\overline{\mathrm{I}})-\chi(\overline{\mathrm{V}}) S(\mathrm{III}) \delta u \otimes \widehat{\delta l}+(\overline{\mathrm{V}}) S(\mathrm{III}) S(\overline{\mathrm{I}}) .
\end{aligned}
$$

The first term in the right-hand side of (C.7) is $O\left(\mu^{3} \chi\right)$, using Corollary C.11 (1). Next,

$$
\delta l(\mathrm{III}) S(\overline{\mathrm{I}})=\delta l(\mathrm{III}) S N_{1}+\chi \delta l(\mathrm{III}) S u \otimes \hat{l}=O(\mu) .
$$

This implies that the second term in the right-hand side of (C.7) is $O(\mu \chi)$. To consider the third term in the right-hand side of (C.7), we note that

$$
(\overline{\mathrm{V}}) S(\mathrm{III}) \delta u=N_{5} S(\mathrm{III}) \delta u+\chi \hat{u} \otimes l^{\prime} \cdot S(\mathrm{III}) \delta u=O(\mu) .
$$

So, the third term is also $O(\mu \chi)$. Thus, we get

$$
(\mathrm{C} .7)=(\overline{\mathrm{V}}) S(\mathrm{III}) S(\overline{\mathrm{I}})+O(\mu \chi) .
$$

We need the following calculations.

Sublemma C.12. We have the following estimates, as $1 / \chi \ll \mu \rightarrow 0$ :

(1) $l^{\prime} S N_{1}, l^{\prime} N_{3} S N_{1}, l^{\prime} S N_{3} S N_{1}=\left(1,0_{1 \times 9}\right)+O(\mu) \rightarrow \hat{\overline{\mathbf{l}}}_{j}$;

(2) $N_{5} S u, N_{5} N_{3} S u, N_{5} S N_{3} S u=\left(0,1,0_{1 \times 8}\right)^{T}+O(\mu) \rightarrow \widetilde{w}$;

(3) $l^{\prime} S u, l^{\prime} S \tilde{u}, l^{\prime} S N_{3} S u \lesssim \mu / \chi$ and $\tilde{l} S u=O\left(1 / \chi^{2}\right)$;

(4) $N_{5} S N_{3} S N_{1}=O(\mu \chi)$.

Proof. Items (1) and (2) can be obtained by taking the limit $\lim _{\mu \rightarrow 0, \chi \rightarrow \infty}$ using Mathematica. In item (4), we use Mathematica to get

$$
\lim _{\substack{\mu \rightarrow 0 \\ \chi \rightarrow \infty}} \frac{N_{5} S N_{3} S N_{1}}{\chi}=0 .
$$

To understand (1) and (2) heuristically, we notice that all the entries of $l^{\prime}$ are small except the first one, so multiplying $l^{\prime}$ by a matrix corresponds to picking out the first row. Though $N_{1}$ and $N_{3}$ have some large entries of order $O(\mu \chi)$, the corresponding entries of $l^{\prime}$ are small enough to suppress them. The first rows of the matrices $S, N_{3}$ and $N_{1}$ all have a similar structure to $l^{\prime}$. Therefore, we may think of $l^{\prime}$ as a left eigenvector of the matrices. The same heuristic argument applies to $u$. To see where (4) comes from, we may think of $S$ as the identity. The big entries of $O(\mu \chi)$ in $N_{1}, N_{3}$ and $N_{5}$ are 
off-diagonal. It is not hard to keep track of these $O(\mu \chi)$ entries to see that we do not get terms greater than $O(\mu \chi)$.

Next, we multiply $(\overline{\mathrm{V}}) S(\mathrm{III}) S(\overline{\mathrm{I}})$ to get

$$
\begin{aligned}
{\left[N_{5}+\chi \hat{u} \otimes l^{\prime}\right] S\left[N_{3}+\chi \tilde{u} \otimes l^{\prime}+\chi u \otimes \tilde{l}\right] S\left[N_{1}+\chi u \otimes \hat{l}\right] } \\
=\left[N_{5}+\chi \hat{u} \otimes l^{\prime}\right]\left[S N_{3}+\chi S \tilde{u} \otimes l^{\prime}+\chi S u \otimes \tilde{l}\right]\left[S N_{1}+\chi S u \otimes \hat{l}\right] \\
=\left[N_{5} S N_{3}+N_{5}\left(\chi S \tilde{u} \otimes l^{\prime}+\chi S u \otimes \tilde{l}\right)+\chi \hat{u} \otimes l^{\prime} S N_{3}+O(\mu \chi) \hat{u} \otimes l^{\prime}+O(\mu)\right] \\
\quad \times\left(S N_{1}+\chi S u \otimes \hat{l}\right) \\
=N_{5} S N_{3} S N_{1}+\chi N_{5} S \tilde{u} \otimes l^{\prime} S N_{1}+\chi N_{5} S u \otimes \tilde{l} S N_{1}+\chi \hat{u} \otimes l^{\prime} S N_{3} S N_{1} \\
\quad+O(\mu \chi) \hat{u} \otimes l^{\prime} S N_{1}+\chi N_{5} S N_{3} S u \otimes \hat{l}+N_{5}\left(\chi S \tilde{u} \otimes l^{\prime}+\chi S u \otimes \tilde{l}\right)(\chi S u \otimes \hat{l}) \\
\quad+\left(\chi \hat{u} \otimes l^{\prime}\right) S N_{3}(\chi S u \otimes \hat{l})+O\left(\mu \chi^{2}\right) \hat{u} \otimes l^{\prime} \cdot S u \otimes \hat{l}+O(\mu \chi),
\end{aligned}
$$

where, in the second equality, we use $l^{\prime} S u \sim l^{\prime} S \tilde{u} \lesssim \mu / \chi$, by Sublemma C.12(3), and $\tilde{l}=l^{\prime}+O(1 / \chi)$, by their definitions in Definition C.2 and Proposition 5.2 (a.2).

The first term in (C.8) is $O(\mu \chi)$, by Sublemma C.12(4). The ninth term

$$
\mu \chi^{2} \hat{u} \otimes l^{\prime} \cdot S u \otimes \hat{l}=O\left(\mu^{2} \chi\right)
$$

since $l^{\prime} \cdot S u=O(\mu / \chi)$ by Sublemma C.12 (3). The seventh term

$$
N_{5}\left(\chi S \tilde{u} \otimes l^{\prime}+\chi S u \otimes \tilde{l}\right)(\chi S u \otimes \hat{l})=O(\mu \chi)
$$

using that $\tilde{u}=u+O(1 / \chi)$ and Sublemma C.12(3). The fifth term has the estimate

$$
\mu \chi \hat{u} \otimes l^{\prime} S N_{1}=O(\mu \chi)
$$

by Sublemma C.12(1). The eighth term

$$
\left(\chi \hat{u} \otimes l^{\prime}\right) S N_{3}(\chi S u \otimes \hat{l})=O(\mu \chi)
$$

since $l^{\prime} S N_{3} S u \lesssim \mu / \chi$ by Sublemma C.12 (3).

We are left with four terms, the second, third, fourth and sixth terms, written together as

$$
\chi\left[N_{5} S \tilde{u} \otimes l^{\prime} S N_{1}+N_{5} S u \otimes \tilde{l} S N_{1}+\hat{u} \otimes l^{\prime} S N_{3} S N_{1}+N_{5} S N_{3} S u \otimes \hat{l}\right] .
$$

We first use the fact that

$$
\tilde{u}=u+O\left(\frac{1}{\chi}\right), \quad \tilde{l}=l+O\left(\frac{1}{\chi}\right), \quad l^{\prime}=l+O\left(\frac{1}{\chi}\right), \quad \hat{l}=l+O(\mu)
$$


and $N_{1}, N_{5}=O(\mu \chi)$ to reduce the four terms to

$$
\chi\left[2\left(N_{5} S u+O(\mu)\right) \otimes\left(l^{\prime} S N_{1}+O(\mu)\right)+\hat{u} \otimes l^{\prime} S N_{3} S N_{1}+N_{5} S N_{3} S u \otimes\left(l^{\prime}+O(\mu)\right)\right] .
$$

Using parts (1) and (2) of Sublemma C.12, we find that each term in expression (C.9) has the form of

$$
\chi(u+O(\mu)) \otimes\left(l^{\prime}+O(\mu)\right)=\chi u \otimes l^{\prime}+O(\mu \chi) .
$$

Up to now, we have successfully separated the $O\left(\chi^{2}\right), O(\chi)$ and $O(\mu \chi)$ parts in the global map.

Step 5. (Completing the proof.)

Remember we have dropped the two $O(1)$ matrices $\left(1+u_{1}^{\mathrm{i}} \otimes l_{1}^{\mathrm{i}}\right)$ in (I) and $\left(1+u_{5}^{\mathrm{f}} \otimes l_{5}^{\mathrm{f}}\right)$ in (V) in Step 0. We summarize the results of Steps 2 and 4 as follows:

$$
d \mathbb{G}=\left(\operatorname{Id}_{10}+u_{5}^{\mathrm{f}} \otimes l_{5}^{\mathrm{f}}\right)\left(\chi^{2} \bar{u}^{\prime} \otimes \bar{l}^{\prime}+\chi u \otimes l^{\prime}+O(\mu \chi)\right)\left(\operatorname{Id}_{10}+u_{1}^{\mathrm{i}} \otimes l_{1}^{\mathrm{i}}\right) .
$$

To complete the proof of the lemma, it is enough to define

$$
\begin{array}{ll}
\overline{\mathbf{u}}=\left(\operatorname{Id}_{10}+u_{5}^{\mathrm{f}} \otimes l_{5}^{\mathrm{f}}\right) \bar{u}^{\prime}, & \overline{\overline{\mathbf{u}}}=\left(\operatorname{Id}_{10}+u_{5}^{\mathrm{f}} \otimes l_{5}^{\mathrm{f}}\right) u, \\
\overline{\mathbf{l}}=\bar{l}^{\prime}\left(\operatorname{Id}_{10}+u_{1}^{\mathrm{i}} \otimes l_{1}^{\mathrm{i}}\right), & \overline{\overline{\mathbf{l}}}=l^{\prime}\left(\operatorname{Id}_{10}+u_{1}^{\mathrm{i}} \otimes l_{1}^{\mathrm{i}}\right) .
\end{array}
$$

We obtain the structure of $d \mathbb{G}$ stated in Lemma 3.2. It remains to work out the vectors $\overline{\overline{\mathbf{u}}}, \overline{\mathbf{u}}, \overline{\overline{\mathbf{l}}}$ and $\overline{\mathbf{l}}$. We have

$$
\overline{\overline{\mathbf{u}}}=u+O(\mu) \rightarrow\left(0,1,0_{1 \times 8}\right)^{T} \quad \text { and } \quad \overline{\overline{\mathbf{l}}}=l^{\prime}+O(\mu) \rightarrow\left(1,0_{1 \times 9}\right)
$$

as $1 / \chi \ll \mu \rightarrow 0$, using Sublemma C.12 for $u$ and $l^{\prime}$, and Proposition 5.2 for $u_{1}^{\mathrm{i}}$ and $l_{5}^{\mathrm{f}}$. According to (C.4) in Step 2, we have $\bar{u}^{\prime}=N_{5} \hat{u}_{i i i^{\prime}}+O(1) \hat{u}$ and $\bar{l}^{\prime}=\hat{l}_{i} N_{1}$. We neglect the term $O(1) \hat{u}$, since it is enough to consider $\operatorname{span}\left\{N_{5} \hat{u}_{i i i^{\prime}}, \hat{u}\right\}$, and $\hat{u}=u+O(\mu)$ is already provided by the $O(\chi)$ part of $d \mathbb{G}$. Using $\hat{u}_{i i i^{\prime}}$ in Definition C.2 and $N_{1}$ in Proposition 5.2, we find that in $\bar{u}^{\prime}$ we have $N_{5} \hat{u}_{i i i^{\prime}} \rightarrow\left(0, O(1), 0_{1 \times 6}, O(1), O(1)\right)$ as $1 / \chi \ll \mu \rightarrow 0$, where the last two $O(1)$ entries are

$$
\left(N_{5}\right)_{44} \cdot\left(u_{i i i^{\prime}}(9), u_{i i i^{\prime}}(10)\right)^{T}=\left(u_{i i i^{\prime}}(9), u_{i i i^{\prime}}(10)\right)^{T}=\left(1, \frac{\hat{L}_{4}}{\hat{L}_{4}^{2}+\widehat{G}_{4}^{2}}\right),
$$

$\left(\left(u_{i i i^{\prime}}(9), u_{i i i^{\prime}}(10)\right)\right.$ is an eigenvector of $\left(N_{5}\right)_{44}$ with eigenvalue 1$)$, and in $\bar{l}^{\prime}$ we have

$$
\hat{l}_{i} N_{1} \rightarrow \lim l_{i}=\left(\frac{\widetilde{G}_{4}}{\tilde{L}_{4}\left(\tilde{L}_{4}^{2}+\widetilde{G}_{4}^{2}\right)}, 0_{1 \times 7},-\frac{1}{\tilde{L}_{4}^{2}+\widetilde{G}_{4}^{2}},-\frac{1}{\tilde{L}_{4}}\right) .
$$

It is easy to see that $u \rightarrow \overline{\overline{\mathbf{u}}}$, using the definition of $u$ in Proposition 5.2. We substitute these calculations back to (C.10) to get $\overline{\mathbf{u}} \rightarrow u_{i i i^{\prime}}+c \overline{\overline{\mathbf{u}}}$ for some constant $c$. 


\section{Acknowledgement}

The author would like to thank his thesis advisor Prof. Dmitry Dolgopyat, who spent one year checking all the details of the paper. Without his enormous intelligence and time input, constant encouragement and financial support, the work could not have been completed. The author would also like to express his deep gratitude to Professor Joseph Gerver, who also carefully checked all the details of the paper and gave numerous suggestions which significantly improve the readability of the paper. The author is supported by NSFC (Significant project No.11790273) in China and Beijing Natural Science Foundation (Z180003).

\section{References}

[DX] Dolgopyat, D. \& Xue, J., Non-collision singularities in the planar two-center-two-body problem. Comm. Math. Phys., 345 (2016), 797-879.

[F] Floria, L., A simple derivation of the hyperbolic Delaunay variables. The Astronomical Journal, 110 (1995), 940-942.

[G1] Gerver, J. L., The existence of pseudocollisions in the plane. J. Differential Equations, 89 (1991), 1-68.

[G2] - Noncollision singularities: do four bodies suffice? Experiment. Math., 12 (2003), 187198.

[G3] - Noncollision singularities in the n-body problem, in Dynamical Systems. Part I, Pubbl. Cent. Ric. Mat. Ennio Giorgi, pp. 57-86. Scuola Norm. Sup., Pisa, 2003.

[MM] Mather, J. N. \& McGehee, R., Solutions of the collinear four body problem which become unbounded in finite time, in Dynamical Systems, Theory and Applications (Seattle, WA, 1974), Lecture Notes in Phys., 38, pp. 573-597. Springer, BerlinHeidelberg, 1975.

[P] Painlevé, P., Leçons sur la théorie analytique des équations différentielles. Hermann, Paris, 1897.

[Sa1] SAARI, D. G., Improbability of collisions in Newtonian gravitational systems. Trans. Amer. Math. Soc., 162 (1971), 267-271; erratum, ibid. 168 (1972), 521.

[Sa2] - A global existence theorem for the four-body problem of Newtonian mechanics. $J$. Differential Equations, 26 (1977), 80-111.

[Si] Simon, B., Fifteen problems in mathematical physics, in Perspectives in Mathematics, pp. 423-454. Birkhäuser, Basel, 1984.

[Su] Sundman, K. F., Nouvelles recherches sur le problème des trois corps. Acta. Soc. Sci. Fennicae, 35 (1909), 3-27.

[W] Wintner, A., The Analytical Foundations of Celestial Mechanics. Princeton Mathematical Series, Vol. 5. Princeton Univ. Press, Princeton, NJ, 1941.

[Xi] XIA, Z., The existence of noncollision singularities in Newtonian systems. Ann. of Math., 135 (1992), 411-468. 
JinXin XuE

Yau Mathematical Sciences Center

\& Department of Mathematics

Tsinghua University

Beijing, China, 100084

jxue@tsinghua.edu.cn

Received September 14, 2014 